\title{
NISTIR 88-3888
}

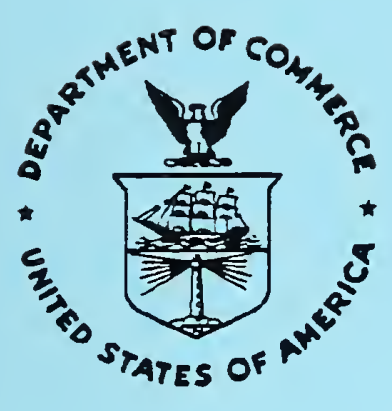

\section{Summaries of Center for Fire Research In-House Projects and Grants - 1988}

Sonya M. Cherry, Editor

\section{U.S. DEPARTMENT OF COMMERCE}

National Institute of Standards and Technology

(Formerly National Bureau of Standards)

National Engineering Laboratory

Center for Fire Research

Gaithersburg, MD 20899

November 1988

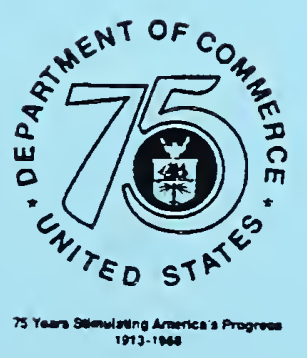




\section{.}




\section{NISTIR 88-3888}

\section{Summaries of Center for Fire Research In-House Projects and Grants - 1988}

Sonya M. Cherry, Editor

U.S. DEPARTMENT OF COMMERCE

National Institute of Standards and Technology

(Formerly National Bureau of Standards)

National Engineering Laboratory

Center for Fire Research

Gaithersburg, MD 20899

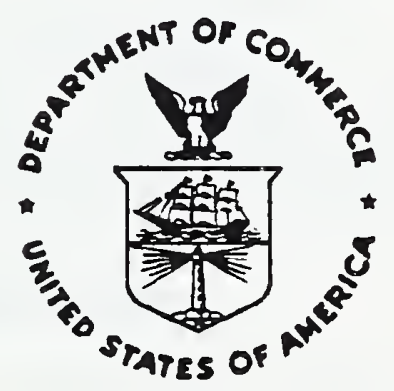

National Bureau of Standards became the National Institute of Standards and Technology on August 23, 1988, when the Omnibus Trade and Competitiveness Act was signed. NIST retains all NBS functions. Its new programs will encourage improved use of technology by U.S. industry.

U.S. DEPARTMENT OF COMMERCE

C. William Verity, Secretary

NATIONAL INSTITUTE OF STANDARDS

AND TECHNOLOGY

Ernest Ambler, Director 

PART I. Basic Research and Tools for "Engineered" Fire Safety (In-House Projects and Associated Grants Funded by NIST)

A. Fire/Materials Interaction

Polymer Gasification (in-house)

Flame Radiation (grant)

Interaction of Radiation and Conduction in Polymeric Materials (grant)

A Study of the Effects of Oxygen Transport and Temperature History on the Chemistry of PMMA and Cellulose Pyrolysis (grant)

Thermal Stabilization of Polymers by CrossLink Formation (grant)

Computer-Aided Molecular Design of Materials with Improved Fire-Safety Characteristics (in-house)

Flame Spread (in-house)

Prediction of Fire Dynamics (grant)

Fire Propagation in Concurrent Flows (grant) Wind-Aided Flame Spread (grant)

Carbon Monoxide Generation From Smoldering Combustion (in-house)

Kinetics of Oxygen Chemisorption and Desorption of Combustion Products in the Smoldering Combustion of Cellulosic Material (grant)

B. Flame Dynamic Processes

Turbulent Combustion Simulation (in-house) 61

Soot Formation and Evolution (in-house) 65

Soot Morphology in Flames (grant) 69

Chemical Pathways to Soot Formation in Diffusion Flames (grant)

Cooperative Research in the Kinetics of Aggregate Formation (grant) 77

Fire Modeling (grant)

C. Compartment Fire Modeling

Wall Fire Growth in Enclosures (in-house) 
Consolidated Compartment Fire Model (in-house)

Compartment Fire Combustion Dynamics (grant)

Experimental Study of the Environment and Heat Transfer in a Room Fire (grant)

Negatively-Buoyant Flows Generated in Enclosure

Fires by the Downward Turning of Ceiling Jets (grant)

Numerical Analysis Support for Compartment Fire Modeling Code Development (grant)

Model Accuracy Assessment (in-house)

Graphical Presentation and Numerical Analysis of Fire Data for Model Validation (grant)

D. Hazard Modeling

Carbon Monoxide Prediction (in-house)

Soot Particle Formation and Destruction in Diffusion Flames (grant)

Radiation from Turbulent Luminous Flames (grant)

Smoke Transport (in-house)

Toxic Potency Measurement (in-house)

Analysis of Hazards to Life Safety in Fires (grant)

Toxicity of Plastic Combustion Products (grant)

Expert System Approach for Spectra/Structure Correlation for Vapor-Phase Spectra (grant)

Fire Data for Materials (in-house)

Cone Calorimeter Development (in-house)

Fire Hazard Assessment (in-house)

\section{E. Fire Protection Technology}

Engineering Analysis System \& Fire Reconstruction (in-house)

Fire Suppression (in-house)

Effect of Water on Ignition of Cellulosic Materials (grant)

Transient Cooling of A Hot Surface by Droplets Evaporation (grant)

A Study of the Suppression and Extinction of Fires by Water Sprays (grant)

Development of a Technique to Assess the Adequacy of the Municipal Water Supply for Residential Sprinkler Systems (grant) 
F. Technology Transfer

Technology Transfer (in-house)

PART II. Timely Response to Current Fire Problems

(Projects Funded by Other Agencies and Private Sector Organizations)

\section{A. Fire/Materials Interaction}

Radiative Ignition in Microgravity Environment (NASA)

Low Flammability Composites (Navy)

Fire Properties of Candidate Materials for Composite Deckhouse (Navy)

Burning Characteristics of Combat Ship Compartments and Vertical Fire Spread (Navy)

Prediction of Thermal Degradation and Heat Release from Aircraft Composites (FAA)

Coefficient of Variation of ASTM E-648 Test for Carpets (Carpet \& Rug Institute, American Textile Manufacturers Institute, and The Man Made Fiber Producers Association)

NFPA 701 Multiple Layer Drapery Flammability (ATMI, MMFPA, Wool Bureau)

Fire Performance and Toxicity of Composite Materials (Navy)

A Technical Assessment of Explosive Vapor Detection Devices (LESEL/NIST)

Fire Condition Effects on Smoke Generation and Toxicity (Industry Coalition)

Heat Flux Sensor Calibration (FAA)

B. Smoke Movement and Control

Fire Growth/Suppression within Aircraft (FAA)

Fire Environment in Counterflow Ventilation (grant)

\section{Fire Protection Technology}

Fire and Thermal Characteristics of Navy Fire

Fighting Trainers (Navy Training Systems Center)

False Alarms from Smoke Detectors (VA and AF)

Safety in Offshore Drilling (MMS)

A Study of Oil-Slick Combustion (grant)

An Investigation of Simulated Oil Well

Blowout Fires (grant)

Quick Response Sprinklers in Office Occupancies (GSA) 
Foam Fire Suppression (USFA)

Compartment Fire Model to Simulate the Effects of Roof Vents, Sprinklers, and Their Interactions

(AAMA Research Foundation)

Expert System (AF)

Fire Risk Assessment (NFPRF)

Fire Risk Analysis Methodology (grant) 


\section{PREFACE}

This report describes the research projects performed in the Center for Fire Research (CFR) and under its grants program during FY-1988.

The Center is nationally recognized as the focal point for fire research and is the largest and most comprehensive fire research laboratory in the world, with an extremely competent multi-disciplinary technical staff that is supported by an excellent fire library and extensive laboratory facilities.

The Center was created by the Federal Fire Prevention and Control Act of 1974 which authorized the Secretary of Commerce, through the Center, to conduct a fire research program directly or through contracts or grants. Therefore, in addition to its in-house program, the Center maintains a fire research grants program that supplements most of the in-house programs and supports most of the academic fire research in the country. The Center, as a component of the National Institute of Standards and Technology (formerly the National Bureau of Standards), responds to the needs of other Federal agencies and private sector organizations. This report covers work performed with funds appropriated to the Center and work performed under contract to other agencies.

The goal of the Center's program is to provide the technical basis for the reduction of losses and costs of fire. The program is designed to upgrade the knowledge of fire and the production of usable products to meet the fire safety needs of the public, the fire services, and the manufacturing industry. It is comprised of three elements:

- Basic research leading to the development of tools for "engineered" fire safety; i.e., fire protection technologies and fire prediction methods as demonstrated in such CFR fire models as: FIREFOAM, HAZARD, and CCFM.

- Timely response to current fire problems; for example, smoke toxicity, hazards of upholstered furniture, investigations support and fire reconstructions.

- Serves as a focal point for fire research in the nation by incorporating in its program academic and foreign Guest Researchers, collaborating and cooperating with international work in fire research, conducting fire simulation lab workshops; as well as technology transfer and support activities such as standards and codes participation, FRIS, the Computer Bulletin Board, NFRSC, meetings, etc.

(Note: For FY-89, the Center's program has been expanded in the areas of smoke toxicity and upholstered furniture flammability. Progresis on these projects will be reported in next year's report.)

The majority of our in-house priority projects and grants work fits into the first category, basic research and tools for engineered fire safety, and is 
presented in Part I of this report. Our work for other agencies fits in the second, timely response to current fire problems, and is presented in Part II. Activities under the third element are diverse and are not included in this report.

Part I is organized to reflect the way in which the grants augment and support the in-house program. Therefore, each grant report is presented immediately following its related in-house priority project. The projects are arranged in five groups:

\author{
Fire/Materials Interaction \\ Fire Dynamic Processes \\ Compartment Fire Modeling \\ Hazard Modeling \\ Fire Protection Technology \\ Technology Transfer
}

The projects for other agencies, presented in Part II, although usually related to internally funded projects, are primarily designed to meet the missions of those agencies and organizations. The distribution of subjects prevents them from being classified in the same way as those in Part I so they have been organized into three groups:

Fire/Materials Interaction

Smoke Movement and Control

Fire Protection Technology

For the convenience of the reader, an alphabetical listing of all grants is contained in the Appendix.

Most of the material in this report was written before the National Bureau of Standards (NBS) was renamed the National Institute of Standards and Technology (NIST). For purposes of this publication, the names are synonymous. 
PART I. Basic Research and Tools for "Engineered" Fire Safety (In-House Projects and Associated Grants Funded by NIST)

A. FIRE/MATERIALS INTERACTION 



\section{Professional Personnel}

Takashi Kashiwagi, Project Leader

James E. Brown, Research Chemist

Scott Dolan, Chemist

Stephen Fischer, NRC Post Doctorate

Thomas J. Ohlemiller, General Engineer

William J. Parker, Physicist

Kuniyasu Kanemaru, Guest Researcher

Atsumi Omori, Guest Researcher

\section{Project Objectives}

(1) Improve the understanding of the chemical and physical processes which determine flammability characteristics of various types of polymeric materials.

(2) Develop theoretical models to be able to predict their flammability characteristics.

\section{$\underline{\text { Scope }}$}

The approach of this project is basic and aims to: ( 1 ) understand the mechanisms which control material flammability characteristics, (2) determine chemical and physical characteristics of condensed phase and gas phase processes for selected polymeric materials, (3) develop theoretical models to be able to predict material flammability characteristics, and (4) verify the predicted flammability characteristics and physical and chemical quantities with results obtained from well-defined experiments.

\section{Technical Accomplishments}

The effects of thermal stability and melt viscosity on flammability characteristics of polystyrene and poly(methyl methacrylate) were determined by using two different pairs of PS and PMMA samples. The difference in melt viscosity and in thermal stability for these samples was studied by comparing the difference in observed flammability characteristics between the two different initial molecular weight PS or PMMA samples. The difference in the PS samples was based on the effects of melt viscosity and the difference in the PMMA samples was based on the effects of thermal stability and melt viscosity. For this study, a low MW PMMA sample was more stable than a high MW PMMA sample and both low MW samples were less viscose than high MW samples. These effects were determined on piloted ignition, flame spreading, mass burning rate, heat release rate, and global heat of vaporization. The results show that thermal stability of the sample affects piloted ignition delay time, flame spreading rate, mass burning rate, heat release rate, and global heat of vaporization. Melt viscosity of the material affects flame spreading rate and probably mass burning rate at low external radiant flux condition. The 
results for piloted ignition delay time and flame spreading rate are shown in Figures 1 and 2, respectively.

Figure 1. Relationship Between Piloted Ignition Delay Time and Incident External Radiant Flux.
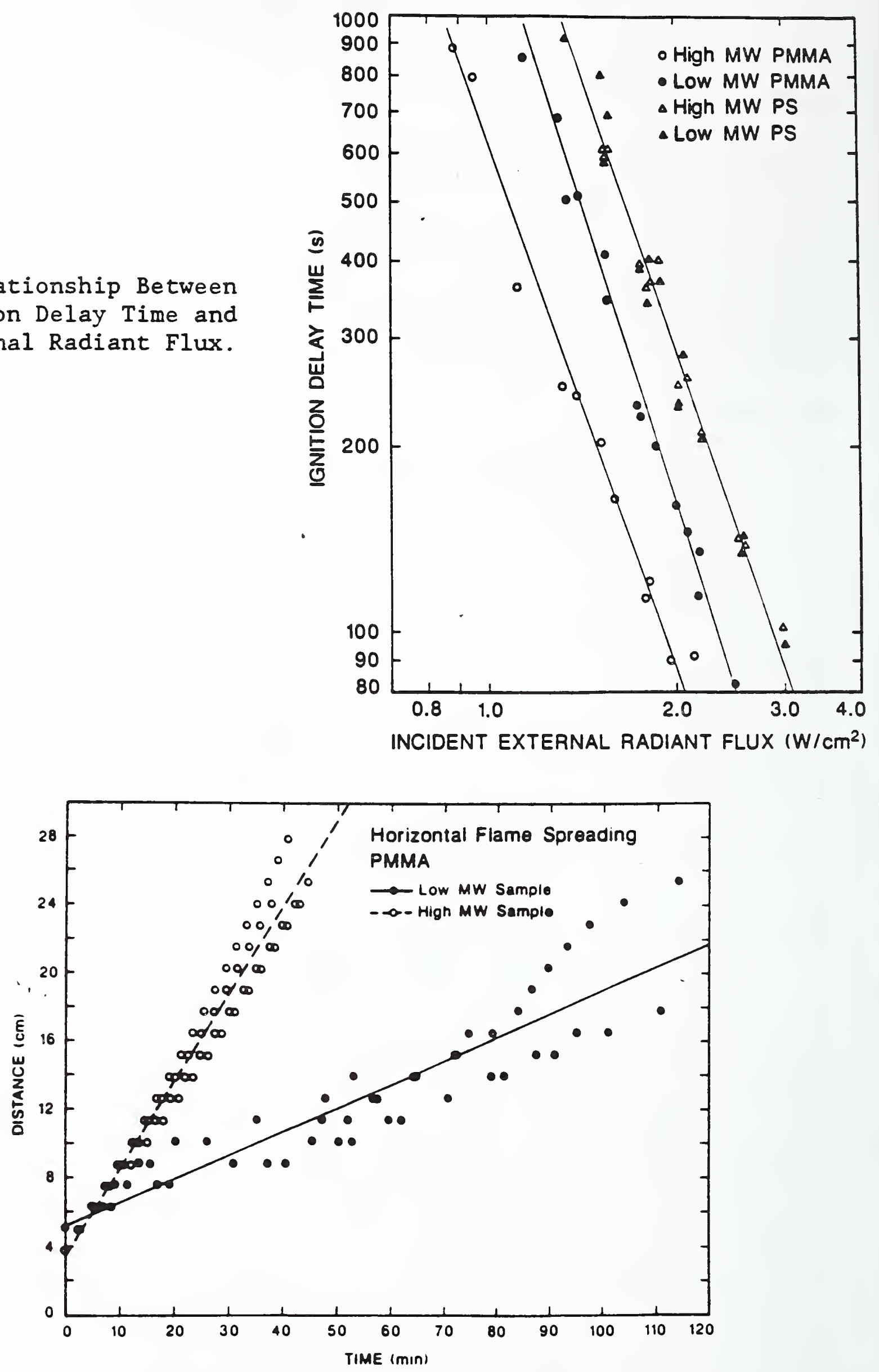

Figure 2. Comparison of Flame Spreading Distance Against Time Between Low MW PMMA and High MW PMMA. 
Changes in molecular weight and in sample weight during thermal degradation of anionically polymerized PMMA samples with different initial molecular weights were calculated numerically and compared with experimental results. The comparison indicates that depropagation from primary radicals as previously thought is questionable and a new degradation path from primary radicals is proposed to describe the behavior of the primary radicals. The primary radical rearranges to form the polymer molecule with the unsaturated bond at a chain end. Two different degradation paths for this rearrangement are proposed via $\beta$ scission at the $C-C$ bond of the pendant group. The specific products evolved from the proposed degradation paths, $\mathrm{CO}, \mathrm{CO}_{2}$, and $\mathrm{CH}_{4}$, were experimentally measured and the experimentally observed trends of these quantities agree well with the theoretically calculated ones.

Measurements have been made on the thermal diffusivity of Douglas fir and its char up to $550^{\circ} \mathrm{C}$. Its char contraction factors have also been determined. Interpretation of some data in the literature has resulted in the establishment of the specific heat as a function of temperature over this range. These thermophysical property data along with some data on the thermochemical properties of cellulose, mannan, xylan and lignin were used as input to the model for the heat release rate of wood in order to calculate the heat release rate of Douglas fir exposed to an external radiant flux of 25 $\mathrm{kW} / \mathrm{m}^{2}$. These calculations are compared in Figure 3 with measurements made in the Cone Calorimeter. The agreement is reasonable at this stage of the model development.

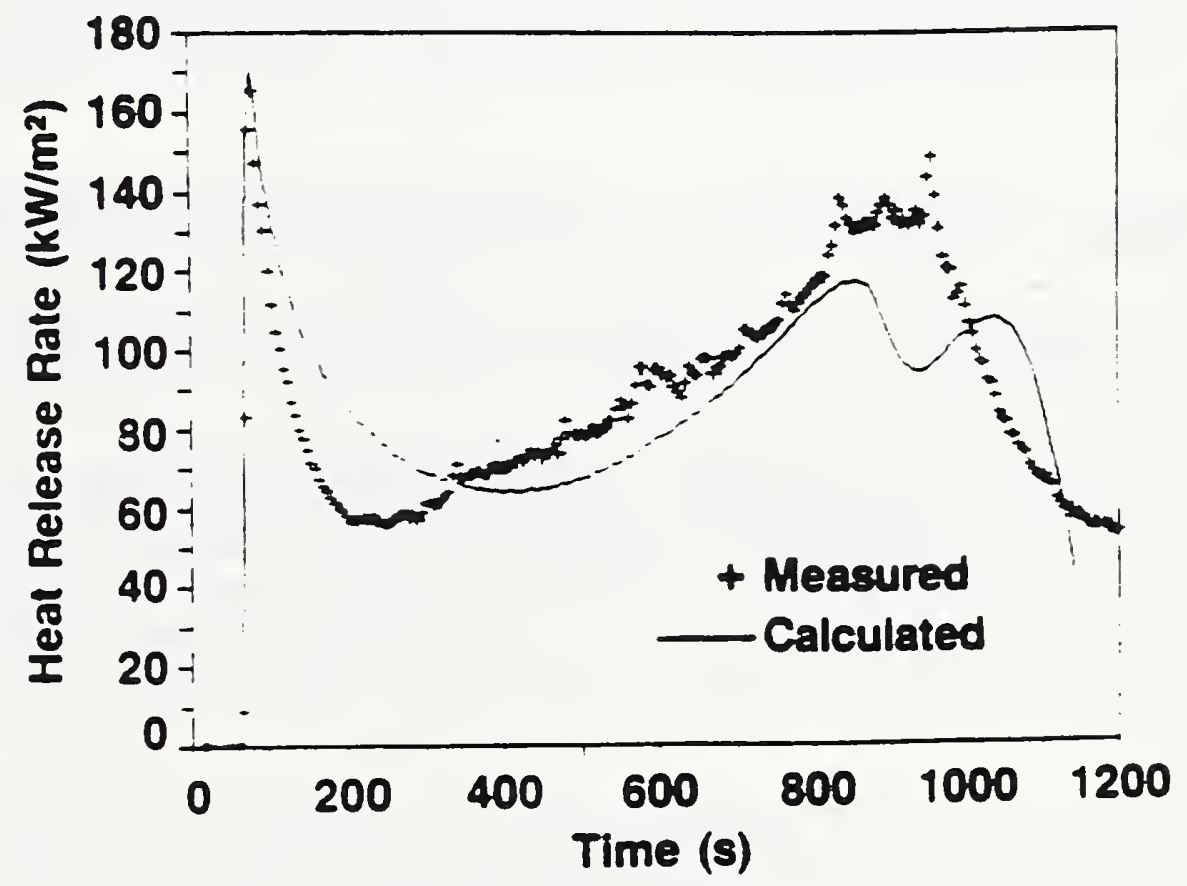

Figure 3. Comparison of Calculated and Measured Heat Release Rate. 
- Inaba, A. and Kashiwagi, T., "A Calculation of Thermal Degradation Initiated by Random Scission, Unsteady Radical Concentration" European Polymer J., 23, 871 (1987).

- Ito, A. and Kashiwagi, T., "Characterization of Flame Spread Over PMMA Using Holographic Interferometry, Sample Orientation Effects" Combustion and Flame, 71, 189 (1988).

- Inaba, A., Kashiwagi, T., and Brown, J.E., "Effects of Initial Molecular Weight on Thermal Degradation of Poly(methyl methacrylate) - 1. Model 1." Polymer Degradation and Stability, 21, 1 (1988).

- Kashiwagi, T., Omori, A., and Brown, J.E., "Effects of Material Characteristics on Flame Spreading" Second International Symposium on Fire Safety Science, 1988 (in press).

- Kashiwagi, T., and Omori, A., "Effects of Thermal Stability and Melt Viscosity of Thermoplastics on Piloted Ignition" Twenty-Second International Symposium on Combustion, 1988 (in press).

- Parker, W.J., "Determination of the Input Data for a Model of the Heat Release Rate of Wood," Mathematical Modeling of Fires, ASTM STP 983, Mehaffey, J.R.,Ed., American Society for Testing and Materials, Philadelphia, 1987, 105-115.

- Parker, W.J., "Prediction of the Heat-Release Rate of Wood", DSc thesis, George Washington, April 1988.

- Parker, W.J., "Prediction of the Heat Release Rate of Douglas Fir", Second International Symposium on Fire Safety Science, 1988 (in press).

\section{Related Grants}

"Flame Radiation", Chang-Lin Tien, University of California, Berkely.

"Interaction of Radiation and Conduction in Polymeric Materials", Wing Aung, University of Maryland.

"A Study of the Effects of Oxygen Transport and Temperature History on the Chemistry of PMMA and Cellulose Pyrolysis", Eric M. Suuberg, Brown University.

"Thermal Stabilization of Polymers by Cross-Link Formation", Charles A. Wilkie, Marquette University. 


\title{
CENTER FOR FIRE RESEARCH \\ NATIONAL BUREAU OF STANDARDS \\ FY 88
}

\author{
Institution: University of California-Berkeley \\ Grant No:: 60NANB4D0018 \\ Grant Title: Flame Radiation \\ Principal Investigator: Dr. Chang-Lin Tien \\ A. Martin Berlin Professor \\ Department of Mechanical Engineering \\ University of California \\ 6179 Etcheverry Hall \\ Berkeley, California 94720 \\ Telephone: (415) 642-0877
}

Other Professional Personnel:

S. Kumar, Post Doctoral

A.J. Stretton, Ph.D. student

S.H. Park, Ph.D. student

NBS Scientific Officer:

A. Tuntomo, M.S. student

Dr. Takashi Kashiwagi

\section{Technical Abstract:}

Introduction. The purpose of this research program is to establish a simple analytical basis for the calculation of radiative heat transfer in fires. From an experimental and theoretical investigation, approxim ate relations have been correlated for a practical engineering application and radiation interactions have been characterized for the verification of the heat transfer mechanism. The present work is focused on three primary topics: (1) experimental and theoretical determination of the infrared radiation properties of hydrocarbon gases pyrolyzed from burning condensed fuels; (2) development of a simplified methodology to investigate radiative transfer phenomena in pool fires and plumes; and (3) experimental and theoretical investigation of the radiation characteristics of agglomerated soot particles.

Radiation Properties of Hydrocarbon Gases. The infrared radiation properties of hydrocarbon gases evolved from burning condensed fuels are important to investigate their blockage effects on the thermal radiation feedback and thus the combustion mechanism of largescale fires. The experimental data of MMA vapor have been taken by a low-resolution infrared radiation measurement apparatus and analyzed to correlate the narrow, wide and super-band parameters [1]. The total emissivity and Planck mean absorption coefficient were calculated with a wide range of optical pathlengths and temperatures for engineering uses. The absorption spectra of the major infrared bands of ethylene have been measured and are being correlated into engineering models [2]. The five principal infrared bands of ethylene are centered near $949.2 \mathrm{~cm}^{-1}(10.5 \mu \mathrm{m}), 1443.5 \mathrm{~cm}^{-1}(6.9 \mu \mathrm{m}), 1889.6 \mathrm{~cm}^{-1}(5.3 \mu \mathrm{m})$, $2325 \mathrm{~cm}^{-1}(4.3 \mu \mathrm{m})$, and $2989.5 \mathrm{~cm}^{-1}(3.35 \mu \mathrm{m})$, as shown in Fig. 1.

Thermal Radiation in Fire Plumes. The thermal radiation heat flux from a pool fire and its associated smoke plume is one of major causes of fire spread and radiation induced ignition. The effects of radiation heat transfer on fire plumes are under investigation with the aid of simple integral formulations.

The importance of thermal radiation in a plume can be viewed by a "radiation" Peclet number $\mathrm{Pe}_{\mathrm{R}}$, which is the ratio of convection energy flux to radiation energy flux at the source of the plume. While thermal radiation plays a dominant role in the fire dynamics, its 
effects are negligible for plumes greater than $10 \mathrm{~m}$ in diameter due to the large value of $\mathrm{Pe}_{\mathrm{R}}$ [3]. Figure 2 shows the effects of cumulatively including various terms to the classical plume model [3] on the plume growth.

The Gaussian profile as an averaged temperature distribution in the conventional integral analysis of smaller plumes generates a false radiation blockage effect at the perimeter of the plume, since the approximate temperature close to the edge of plume structures is lower than the real temperature. The error trends inherent in using time-averaged turbulent properties to predict the radiant heat flux from a moving fluid structure can be demonstrated by a simple analysis of an infinite slab representing a flame layer [4]. Figure 3 shows the possible errors induced by using only the mean property profile for the calculation of radiant heat flux from an homogeneous slab oscillating with a constant amplitude and frequency.

Properties of Soot Agglomerates. The study of radiation properties of soot agglomerates is important to analyze radiative transfer in soot-laden systems and to introduce optical diagnostic methods for inferring particulate properties.

As soot particulates are strong absorbers and emitters of radiation, an accurate determination of their radiative properties is critical to the analysis and prediction of energy tran sport in flames and combustion systems. Due to the difficulties involved in the evaluation of the properties of such agglomerates, an equivalent sphere is introduced as one that exhibits similar radiative properties as the cluster of soot particles comprising the agglomerate [5,6]. A simple analytical reasoning establishes the ratio of the diameter of the effective sphere to the primary particle diam eter to be proportional to the cube root of the number of particles in the cluster [6].

The non-invasive laser diagnostics techniques employed to obtain soot characteristics are sen sitive to the an alytical model selected for the reduction of data. Previous an alyses for data reduction have used the independent assumption whereby each particle in the agglomerate is assumed to act independently of the others in its interactions with the laser beam. This analysis is incapable of predicting the morphological shape of the agglomerated soot particulate. By incorporating the dependent effects [7] in analyzing the scattering from agglomerates it is shown [6] that the scattering pattern from different morphologies is unique and can be used to identify the structure of the agglomerate, as shown in Fig. 4. Hence, the scattering patterns can be a good tool in the optical diagnostics of soot in flames and combustion system $s$ as a data reduction scheme.

\section{Reports and Papers:}

1. Park, S.H., Stretton, A.J., and C.L.Tien,, "Infrared Radiation Properties of Methyl Methacrylate Vapor," Comb. Sci. Tech. (submitted for publication).

2. Tuntomo, A., Park, S.H., and Tien, C.L., "Infrared Radiation Properties of Ethylene," (in preparation).

3. Stretton, A.J., Park, S.H., and Tien, C.L., "Radiation Heat Transfer in Fire Plumes," (in preparation).

4. Stretton, A.J. and Tien, C.L., "Structural-Averaging for Radiation Property Profiles," (in preparation).

5. Drolen, B.L. and Tien, C.L., "Absorption and Scattering of Agglomerated Soot Particulates," J. Quant. Spec. Radiat. Trans. 37, pp. 433-448 (1987).

6. Kumar, S. and Tien, C.L., "Effective Diameter of Agglomerates for radiative Extinction and Scattering," Comb. Sci. Tech. (submitted for publication).

7. Kumar, S. and Tien, C.L., "Dependent Scattering and Absorption od Radiation by Sm all Particles," ASME/AICHE 24th National Heat transfer Conf.(Pittsburg), pp. 9-12 (1987). 


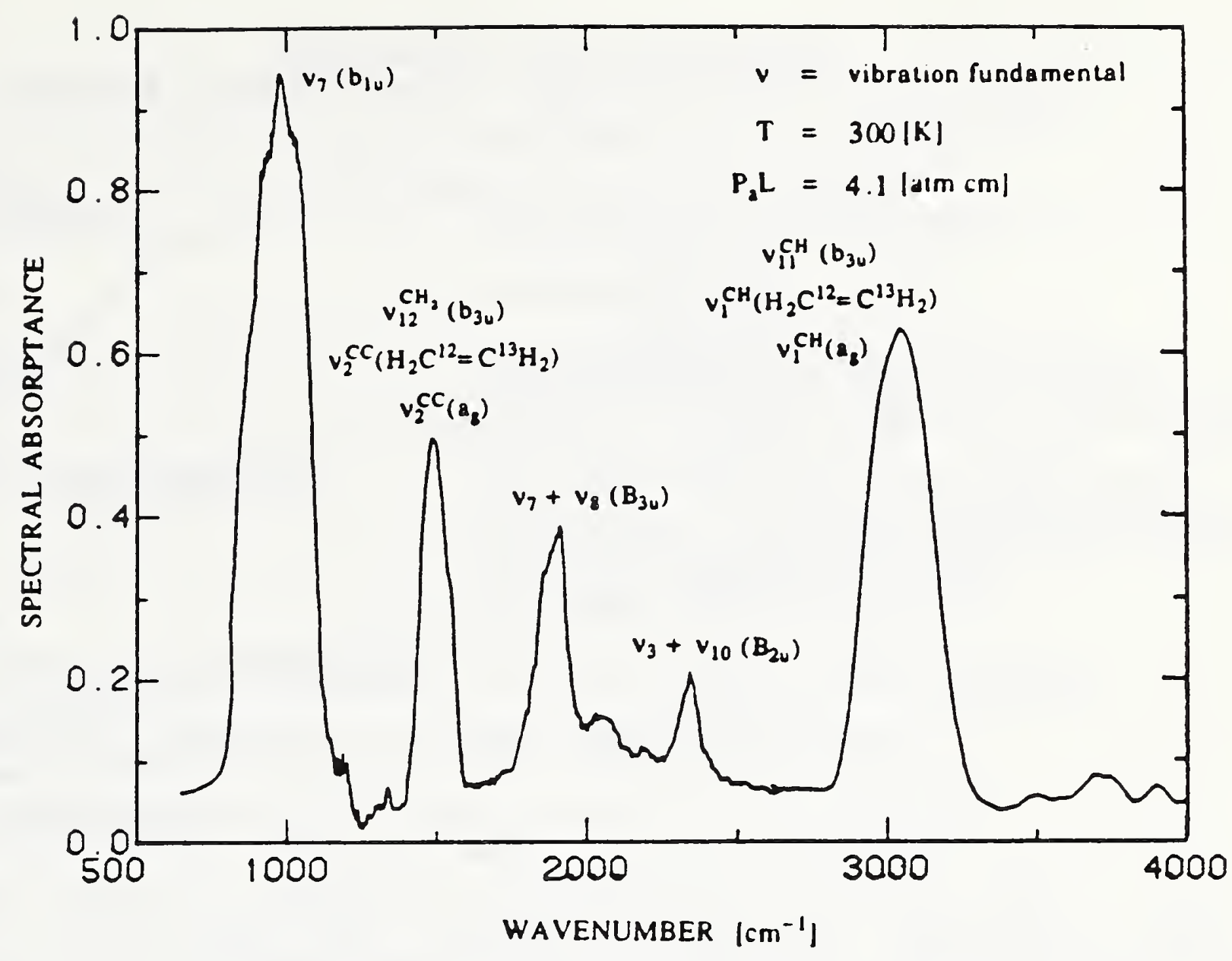

Fig. 1. Spectral absorptance of $\mathrm{C}_{2} \mathrm{H}_{4}$.

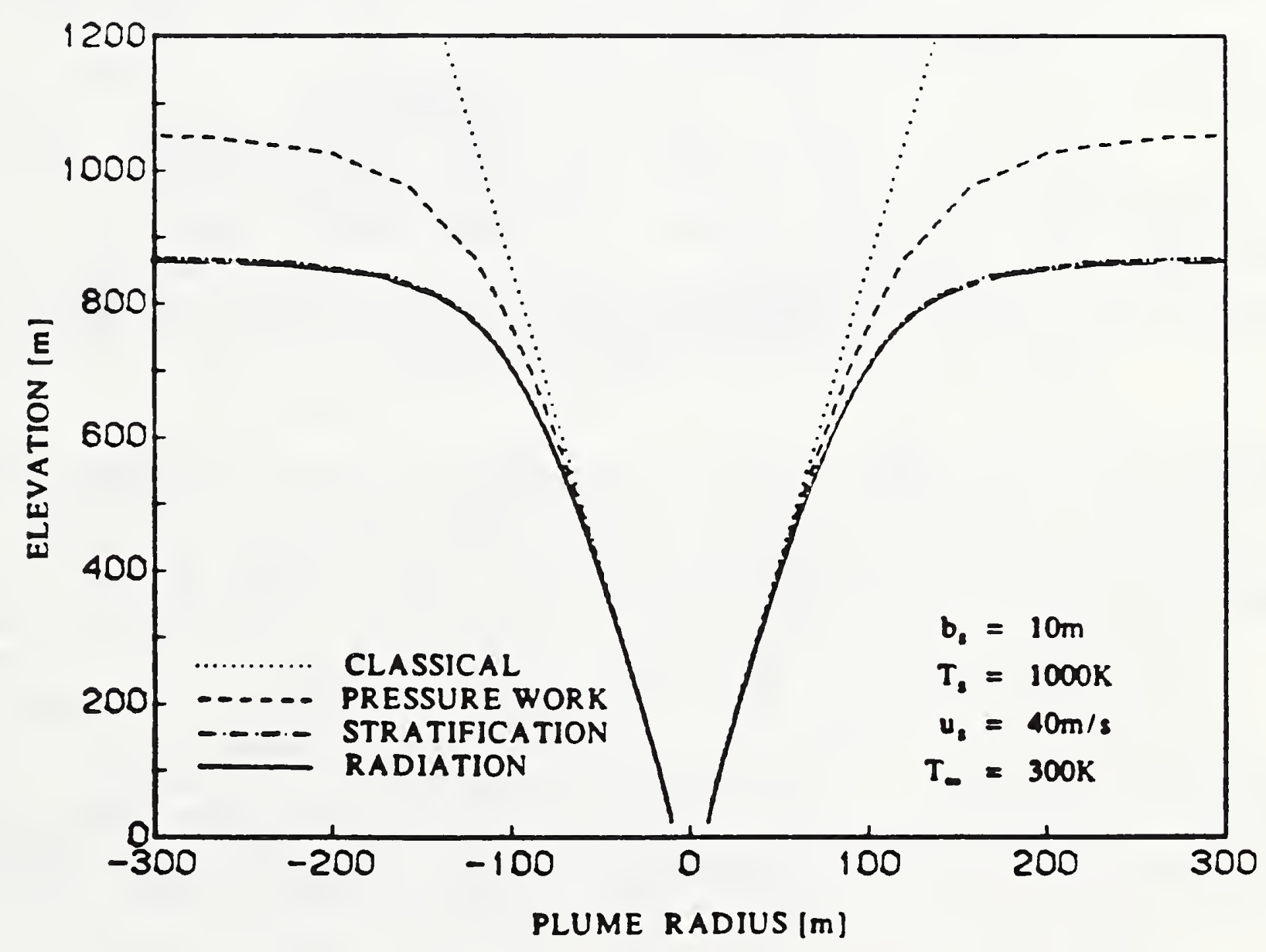

Fig. 2. Comparison of factors affecting fire plume dynamics. 


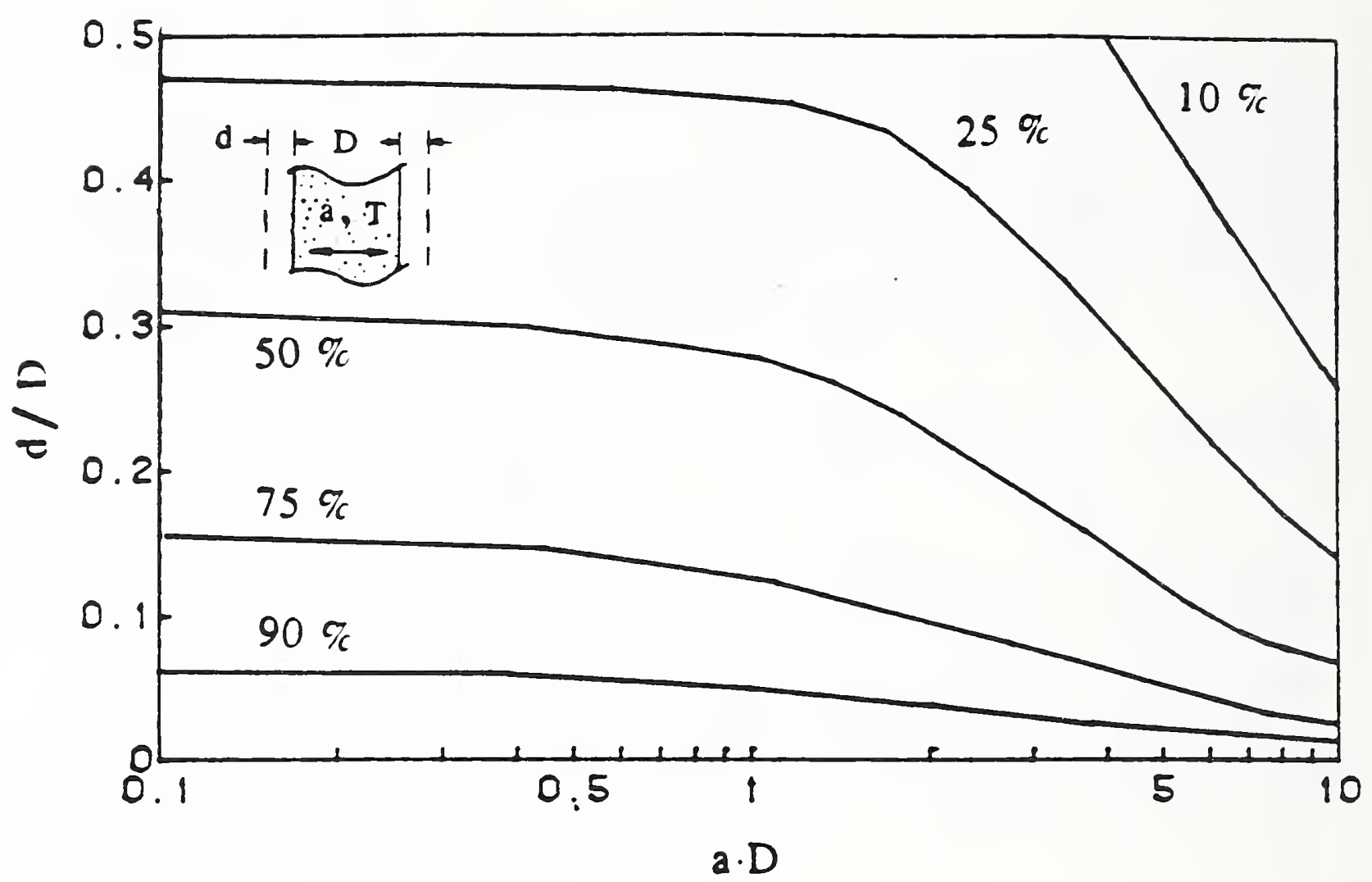

Fig. 3. Mean profile calculation errors for a slab.

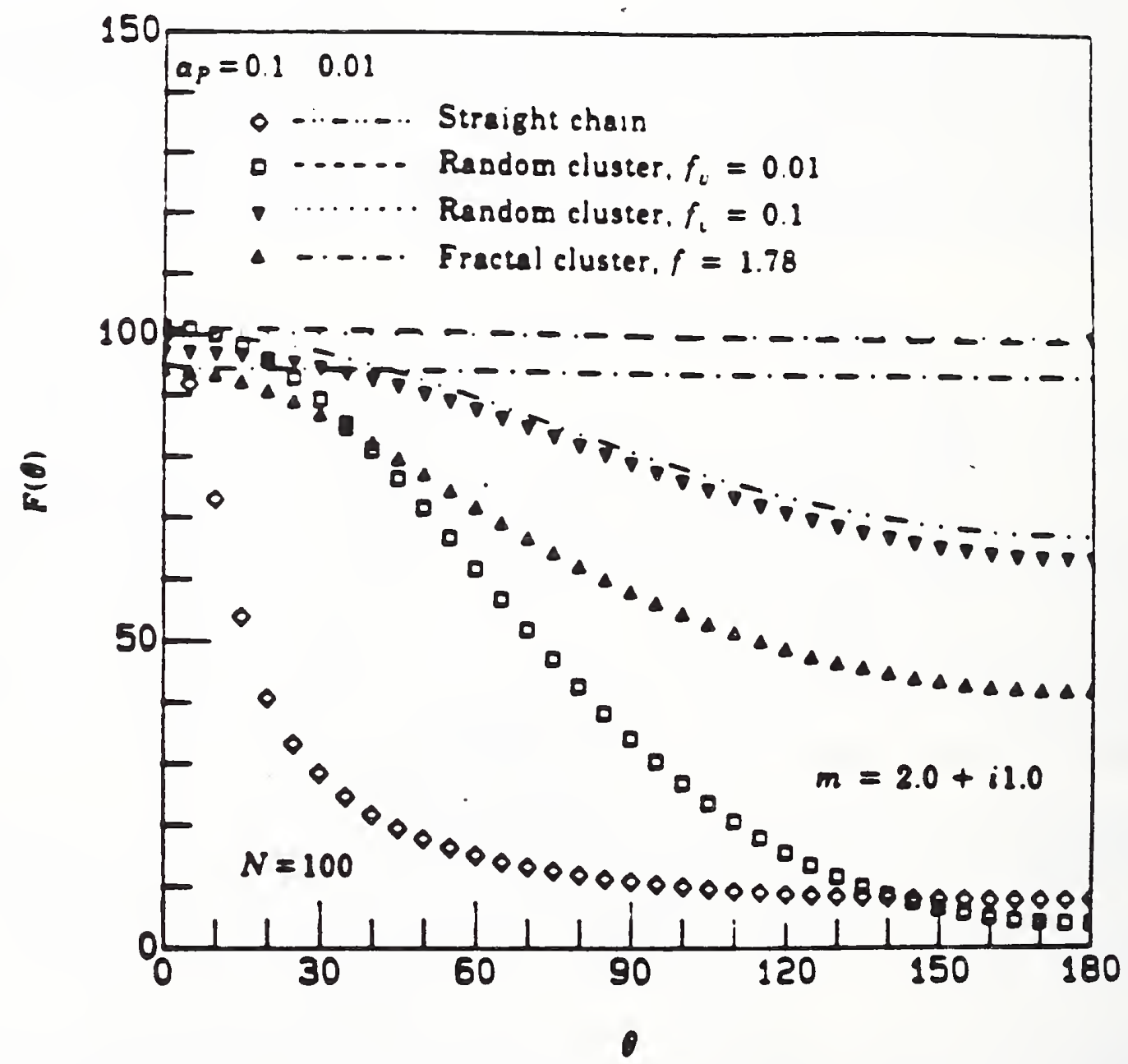

Fig. 4. Scattering pattern of agglomerates. 
Institution:

Grant No.:

Grant Title:

Principal Investigator:
University of Maryland

\section{NANB8H0853}

Interaction of Radiation and

conduction in Polymeric Materials

Dr. Win Aung (Adjunct Professor)

Mechanical Engineering Department

University of Maryland

College Park, MD 20742

Other Professional Personnel: Yen-hao Pan, Ph.D. Student

NBS Scientific Officer: Dr. Takashi Kashiwagi

Technical Abstract:

The objective of this research is to analyze the transient heating of semitransparent polymeric materials exposed to radiant fluxes from high temperature sources. The research is important in fire development because the thermal degradation of polymeric materials may lead to the liberation of flammable gaseous products. To simplify the analysis, we have focused on a onedimension problem. The general model we consider is a vertical plane layer of material with negligible scattering and in local thermodynamic equilibrium. The collimated flux at normal incidence at the irradiated surface of the solid is considered to be a known quantity. The transient energy equation for combined conduction and radiation is solved for boundaries subjected to natural convection and surface radiation. The effects of linearly varying thermal conductivity and specific heat are also considered.

From a heat transfer standpoint, the complete non-flaming, transient heating cycle for a polymeric solid consists of an initial, combined radiative-conductive heating phase involving a homogeneous solid, followed by heating with phase transition and gas-product liberation. Numerical solutions for the initial heating phase have been obtained resulting in development of opaque, cold and re-radiating models. The results of these studies have been summarized by Finlayson, Aung, and Kashiwagi (1987) with details given by Finlayson (1988). In these studies, the re-radiating model has been compared with experimental data taken with fused silica by means of holographic interferometry. Good agreement has been obtained. This model, which solves the coupled nonlinear energy equation and equation of transfer, requires relatively long calculation time, thus diminishing the model's usefulness in the development of the eventual thermochemical model for the second phase heating process. To 
get around this problem, simplifications afforded by the Rosseland approximation have been adopted and the Crank-Nicolson numerical scheme has. also been employed in the present study. Efforts are underway to extend the present transient heating computation scheme to the development of the thermochemical model that will account for phase transformation and chemical reaction.

Rosseland Approximation: A medium is said to be optically thick if the radiation photon mean free path is very small compared with the characteristic dimension of the medium. In the optically thick case, emitted radiation is largely attenuated: hence, energy transfer depends only on the conditions in the immediate vicinity of the position being considered. In the Rosseland approximation the energy transfer is described in terms of the gradient of the conditions at any given position.

Crank-Nicolson Scheme: One other reason for the large time consumption in previous calculations was the use of the implicit scheme. The implicit scheme is un-conditionally convergent, but very small time steps are necessary to maintain accuracy. With a view towards solving this problem, we have adopted the Crank-Nicohlson scheme. The discretization of the energy equation is as following:

$$
\begin{aligned}
\rho c \Delta x\left(T_{P}^{\prime}-T_{P}^{\circ}\right)= & f \Delta t\left[\frac{k_{e}\left(T_{E}^{\prime}-T_{P}^{\prime}\right)}{\Delta x}-\frac{k_{W}\left(T_{P}^{\prime}-T_{W}^{\prime}\right)}{\Delta x}\right] \\
& +(1-f) \Delta t\left[\frac{k_{e}^{\left(T_{E}^{\circ}-T_{P}^{\circ}\right)}}{\Delta x}-\frac{k_{W}\left(T_{P}^{\circ}-T_{W}^{\circ}\right)}{\Delta x}\right]+\int_{t}^{t+\Delta t} \int_{W}^{e} \frac{\partial F}{\partial x} d x d t
\end{aligned}
$$

For an explanation of the symbols used, please see Figure 1.

Results: Rosseland approximation is valid only for locations that are far from boundaries; therefore, the calculated temperature profiles near boundaries are not accurate. To improve this approximation, we have added exponential terms representing "external flux" as source terms in the energy equation. The external flux approach is also used in the cold model (Finlayson et al., 1987; Finlayson, 1988), so the present improved method is actually the "Rosseland + Cold" approach. As indicated in Figure 2 , Rosseland approximation produces acceptable results as compared with experiments. The results displayed in Figure 2 are for fused silica. Sample thickness is $310 \mathrm{~mm}$. The reflectivities of the external flux are determined by the Fresnel equation. The internally emitted radiated heat flux is assumed to be totally reflected at boundaries. The results indicate that the slight sacrifice in accuracy as compared with the reradiating model gives rise to a 50\% reduction in processor time $(800 \mathrm{sec}$ vs $2000 \mathrm{sec}$ on CYBER 205 computer). 
The above results concerned only Rosseland approximation. When the Crank-Nicolson method was coupled into the above, it was discovered that the time step sizes could be increased by up to a factor of ten without noticeable reduction in accuracy. Further research into this is needed, since changes of this magnitude may reflect over-conservativeness in the choice of time step heretofore.

Figure 3 shows a comparison of computations using the new "Cold + Rosseland" model with existing experimental results measured for fused silica. The experimental approach employed is holographic interferometry. The irradiated boundary is subjected to a heat flux of $1.98 \mathrm{~W} / \mathrm{Cm} * 2$. The figure displays the spatial temperature distribution at various times inside the sample. There are five groups of temperature profiles and they correrspond to $30,105,210,375,465$ seconds after the beginning of heating. The results show the both re-radiating and "Cold + Rosseland" models are acceptable as compared with experiments. The "Cold + Rosseland" model is not as accurate as re-radiating model, as mentioned in the previous paragraph, but the former model is certainly an acceptable approach. It is obvious from the figure that, at later times, the calculated temperature profiles are steeper than the experimental results. The discrepancies may be in part due to inaccuracy in specifying the thermal conductivity in the theoretical models, since a higher thermal conductivity leads to a flatter temperature profile. It should be noticed that there are temperature maxima inside the slab, according to the calculations, but this is not evident in the measurements.

Figure 4 shows heat from the front boundary $(x=0)$ for a radiative flux of $2 \mathrm{w} / \mathrm{cm} * 2$. The change in the sign the conducted heat is caused by a change in the direction of the temperature gradient. Initially, the maximum temperature is located at the front boundary itself, and heat conduction occurs from the boundary into the solid and is positive. Later, the location of the maximum temperature shift to the inside of the solid, and heat conduction at the boundary reverses direction. This figure clearly indicates but for a very short duration at the beginning of the heating period, heat conduction transfer occurs in the negative direction, in contrast to the assumption employed in many existing theoretical studies on gas generation in solids.

\section{References}

Finlayson, E.U., Aung, W., and Kashiwagi, T., "Theoretical Models for Combined Radiation-Conduction in semitransparent Solids Heated by External Radiative Flux," Proc. Second ASME/JSME Thermal Engineering Conference,_Vol. 1, pp. 427-432 (1987).

Finlayson, E.U., "Interaction of Conduction and Radiation in Polymeric Materials," M.S. Thesis, Department of Mechanical Engineering, University of Maryland (under preparation). 

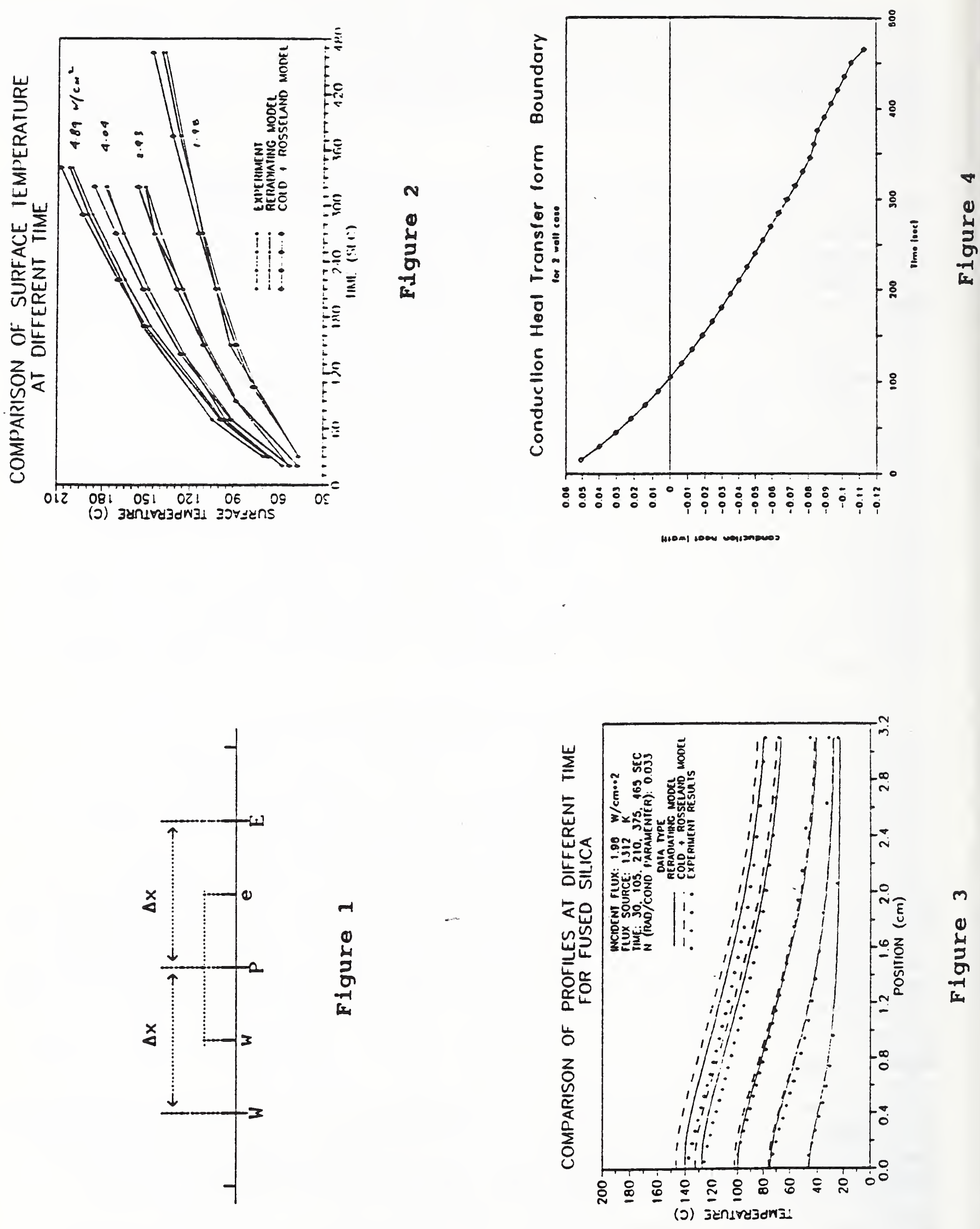
CENTER FOR FIRE RESEARCH
NATIONAL BUREAU OF STANDARDS

FY 88

Institution: Brown University

Grant No.:

60NANB 4D0036

Grant Title:

A Study of the Effects of Oxygen Transport and Temperature History on the Chemistry of PMMA and Cellulose Pyrolysis

Principal Investigator:

Professor Eric M. Suuberg

Division of Engineering

Brown University, Box D

Providence, Rhode Island 02912

Telephone: (401) 863-1420

ether Professional Personnel: Mr. William D. Lilly, Research Engineer

NBS Scientific officer:

Dr. Takashi Kashiwagi

Technical Abstract:

Introduction

The goal of this project is the elucidation of the effects of several key variables on the chemistry of pyrolysis of polymethyl methacrylate (PMMA) and cellulose. These materials are often chosen in fire research as models for vaporizing and charring solids, respectively. The variables studied include: the heating rate of the sample, the partial pressure of oxygen at the surface of the sample, and sample characteristics, such as thickness, or content of flame retardant. Detailed analyses are performed on both the vapor- and condensed-phase products of pyrolysis, and modeling of the processes is conducted in order to determine when they are controlled by mass transfer effects. In the case of PMMA, it is already well established that the major product of pyrolysis is simply the monomer, and that decomposition thus must proceed by a simple "unzipping" process. Gel permeation chromatography (GPC) of the unvaporized polymer residue provides valuable insights into the nature of the process, and the extent to which oxygen affects it at various heating rates.

The conclusions for the part of the project dealing with PMMA will not be discussed at length here, but they may be summarized as follows:

1. Heating rate is not important in determining the global kinetics of PMMA degradation, in the range from $5 \mathrm{~K} / \mathrm{min}$ to $1000 \mathrm{~K} / \mathrm{min}$.

2. The presence of gaseous oxygen at the surface of the sample affects the kinetics of pyrolysis if the heating rate is sufficiently low $(5 \mathrm{~K} / \mathrm{min})$, but when a sample

is heated at a rate of $100 \mathrm{~K} / \mathrm{min}$, the kinetics of decomposition are indistinguishable from those seen in an inert gas. 
In the case of the cellulose pyrolysis process, the products of pyrolysis are much more complex, and consist of many different gas species and tars or oils, as well as a char residue. The effect of the heating rate, oxygen content, and presence of retardant is seen in the mixture of products. The present report will focus on this aspect of the project. Previously, we have already reported the following conclusions concerning the kinetics of cellulose pyrolysis:

1. Variations in heating rates from $5 \mathrm{~K} / \mathrm{min}$ to $400 \mathrm{~K} / \mathrm{sec}$ have a rather minor effect on the global kinetics of cellulose pyrolysis.

2. Oxygen catalyzes decomposition (or more precisely, weight loss) during pyrolysis of thin samples of cellulose, but only at heating rates of $100 \mathrm{~K} / \mathrm{min}$ or less. At higher heating rates, there is apparently not enough opportunity for the oxygen to diffuse into the sample, so that it cannot affect the rate. Whether this is because the surface flux of products is too high to permit oxygen to reach the surface, or the diffusivity of oxygen in the sample is too low for it to be present in significant concentrations, is still under investigation.

\section{Experimental}

All pyrolysis experiments were carried out in a heated wire mesh reactor. The technique involves heating a thin strip of sample (or fine particles) inside of a wire gauze which is suspended between two electrodes in a controlled atmosphere chamber. The wire mesh is resistively heated at a rate which is set in advance and controlled by a microcomputer. The temperature of the mesh is measured by a fine thermocouple inserted in its folds, and this temperature is assumed to be equal to the sample temperature. The products of primary pyrolysis in the polymer (PMMA or cellulose) escape quickly from the porous mesh, and are quenched by mixing with the cold gas in the pyrolysis chamber-consequently the products collected at the end of an experiment are representative of what escapes the surface of the polymer, and are not altered by processes in the vapor phase. The gases are analyzed by gas chromatography. The tars and oils are collected by washing the chamber with an appropriate solvent, and are analyzed by GPC.

Cellulose from two different sources was examined and found to give similar behavior. The first is whatman cellulose powder CF11, medium length fibres, with an ash content of $<0.015 \%$. The diameter of the fibres was in the range 10 to $30 \mu \mathrm{m}$. The second sample was a low ash filter paper-- Munktell's OK/S2-80-200, with an ash content of $<0.007 \%$. This latter sample had a thickness of $168 \pm 8 \mu \mathrm{m}$, and thus was an order of magnitude thicker than the powder, in smallest sample dimension. Nevertheless, there was little significant difference between the powder and paper.

An experiment consists of a complete cycle of heating and cooling the sample. Unlike experiments in a standard thermogravimetric device, in which the mass of the sample is recorded continuously, in the 
present device, the sample mass loss is determined only at the end of the full temperature cycle (the sample mass is measured before and after the experiment). The derivation of kinetic constants requires that samples be heated to various maximum temperatures, and that integrated rate expressions be used for data fitting. The present procedure is thus a bit more tedious than those available for obtaining kinetic constants from TGA data, but the present experimental method has the major advantage that it allows examination of a much wider range of heating rates than can be studied by conventional TGA equipment.

\section{Results and Discussion}

Typical pyrolysis data for samples of unretarded cellulose are shown in Figure 1, obtained at heating rates from $5 \mathrm{~K} / \mathrm{min}$ to $1000 \mathrm{~K} / \mathrm{min}$. In all cases, the cooling rates of the samples were between 200 and $300 \mathrm{~K} / \mathrm{sec}$. Each datum represents the fraction of mass left after the sample was heated to the maximum temperature indicated by the abcissa temperature. Also shown on Fig.1 are calculated kinetic curves, assuming a single, first order global decomposition process, in which the rate of mass loss may be represented by:

$\mathrm{dM} / \mathrm{dt}=-\mathrm{MA} \exp (-\mathrm{E} / \mathrm{RT})$

where $M$ is the mass remaining at time $t, A$ is an Arrhenius pre-exponential factor, $E$ is an activation energy, $R$ the gas constant, and $T$ the temperature. After integration over the full time-temperature history of the sample, it is possible to obtain A and E from data such as shown in Fig. 1. The curves shown were actually calclated from the values of $A$ and $E$ suggested by Lewellwn, Peters, and Howard (16th Symp.on Combustion, p1471,1977), $A=6.79 \times 10^{9} \mathrm{sec}^{-1}$ and $E=33.4 \mathrm{kcal} / \mathrm{mol}$. These values had been obtained by lewellen et al. at heating rates of $400 \mathrm{~K} / \mathrm{sec}$, and show how invariant with heating rate the kinetice of cellulose decomposition are. To fit the data in this case, however required assuming a certain constant small fraction of cellulose always forms char-this fraction was roughly 0.1 .

Attention has recently been turned to the question of how retardant affects the observed pyrolysis behavior of the cellulose. Several experiments were performed at identical conditions, under inert gas, on retarded and unretarded cellulose. The retardant studied in this case was $\mathrm{NaOH}$, wet impregnated into cellulose. The results of the tests performed to date are shown in Table 1 (all at $40 \mathrm{~K} / \mathrm{min}$ heating rate).

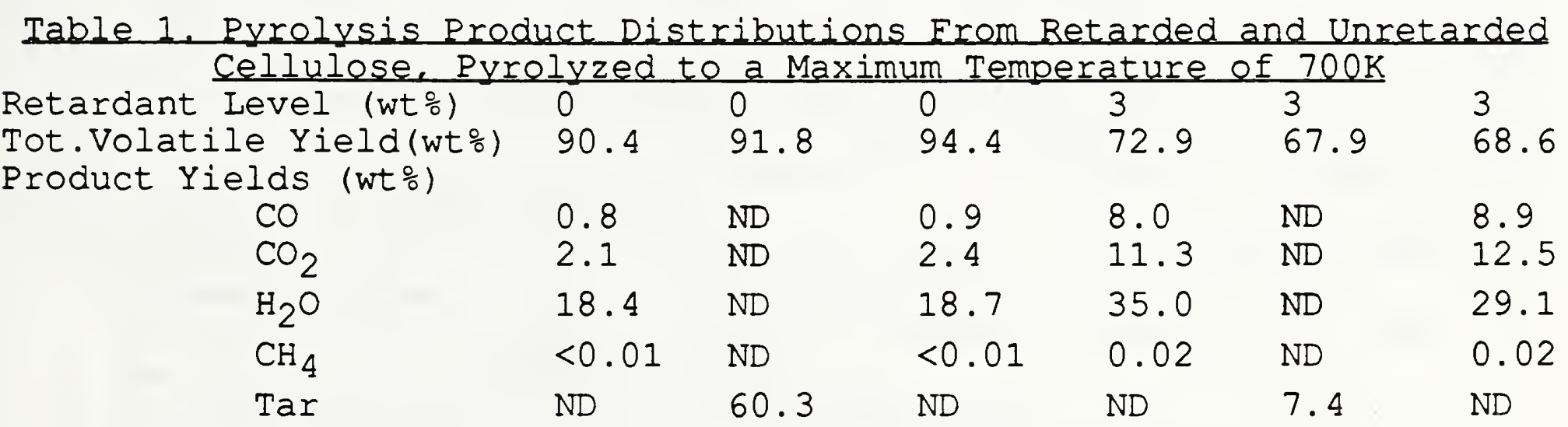

ND-Not Determined 
It is apparent that the data in Table 1 confirm the hypothesized role of retardant in shifting the pathway of pyrolysis away from one which favors formation of flammable tar vapors, to one in which gases with low (or zero) heating value predominate. Retarding a sample would also be expected to result in lowering yields of soot or smoke precursors during non-flaming combustion, because the vaporizing species have an inherently high $0 / C$ ratio in the retarded case.

work is continuing on several aspects of this problem.

\section{Reperts and Papers}

Suluberg,E.M. and Dalal, V., Rrec. Eastern Section of the Combustion Institute, Fall meeting, 1987, pp65-1 to 65-4, Nov.1987.

Suluberg,E.M. and Dalal,V.,"Effect of Heating Rates on the Global Kinetics of Cellulose Pyrolysis", submitted for review for journal publication.

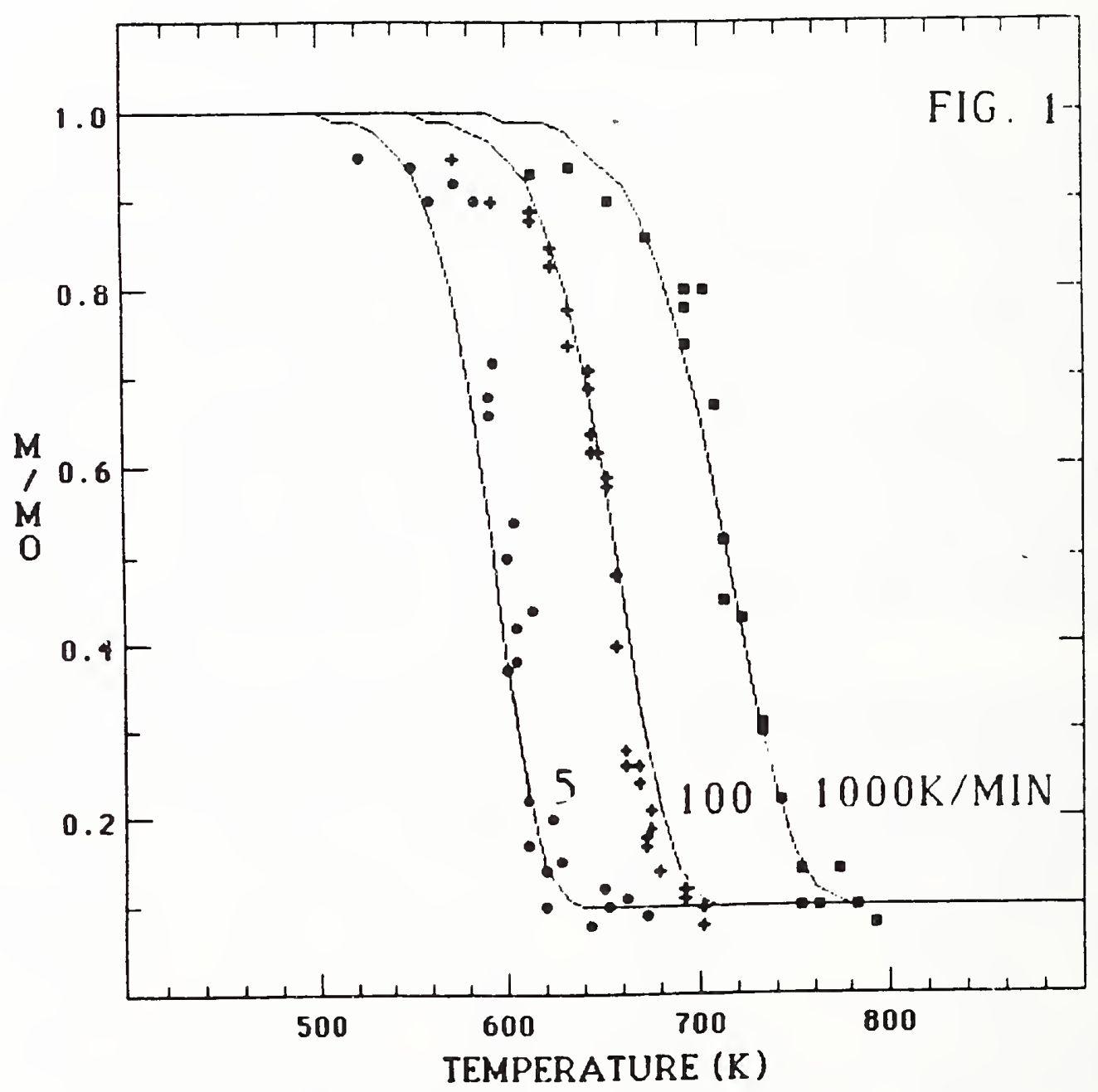




\section{CENTER FOR FIRE RESEARCH \\ NATIONAL BUREAU OF STANDARDS \\ FY88}

Institution:

Grant No.

Title:

Principal Investigator:

Other Personnel:

NBS Scientific Officer:
Marquette University

60NANB6D0602

Thermal Stabilization of Polymers by Cross-Link Formation

Charles A. Wilkie

Department of Chemistry

Marquette University

Milwaukee, WI 53233

Telephone: (414)224-7239

Po-Hui Chang, Graduate Assistant

James Brown

Technical Abstract:

Introduction: The objective of this research program is to understand the chemical basis of pyrolysis reactions of polymers and to use this information to develop suitable schemes for cross-linking of polymers to produce intractable chars. The production of char does not proceed spontaneously, rather additives are required to promote these reactions. Each polymer has a unique set of requirements for the crosslinking reactions and these must be elucidated for each polymer in question. The polymers that are addressed in this research are poly(methyl methacrylate) (PMMA) and poly(ethylene terephthalate) (PET).

\section{Progress to Date}

PMMA. The investigation of Wilkinson's salt, $\mathrm{CIRh}\left(\mathrm{PPh}_{3}\right)_{3}$, as an additive for PMMA has been completed. We can definitely state that this compound does effect cross-linking of the polymer and that this cross-linking leads to an increase in the oxygen index. The reaction proceeds by an oxidative insertion of the rhodium moiety into a carbon-oxygen bond of the polymer. When appropriately configured rhodiuminserted species encounter each other reaction proceeds with the elimination of rhodium-methyl and rhodium-methoxide species and the formation of anhydride. These encounters may proceed within different chains and thus lead to char. Due to problems with expense, color, and toxicity the rhodium compound will never be used as a flame retardant. Analysis of the requirements for an additive to function in this reaction leads to the following conclusions:

1. the additive must be able to interact with carbonyl, i.e. a transition metal compound is required,

2. the additive must be able to participate in oxidative insertion, i.e. it must be coordinatively unsaturated,

3. the compound should show some catalytic activity, and

4. the additives should be colorless and available at a reasonable price. 
Work is underway to identify compounds that fulfill these requirements. Efficacy of the additives will be assessed by oxygen index measurements and thermal analysis techniques as well as from reactions.

In a separate investigation we have probed the suitability of metal carbonyl derivatives as flame retardant additives. The additive that has been studied is $\left[\mathrm{CpFe}(\mathrm{CO})_{2}\right]_{2}$. The observation is that great quantities of gas are produced upon reaction between PMMA and the iron compound, the gas is CO and arises from thermolysis of the iron compound. This reaction is of interest since char formation is also observed. Since this reaction would be of great interest if the evolution of $\mathrm{CO}$ could be suppressed, we have investigated the thermolysis of the dimer. We have delineated the complete reaction pathway which involves an initial dissociation to monomer radicals. The complete reaction pathway is shown in Scheme 1. There are many potential reactants in the decomposition scheme which may interact with the polymer, we have just begun a thorough investigation of the reaction between $\left[\mathrm{CpFe}(\mathrm{CO})_{2}\right]_{2}$ and PMMA and PMMA models.

PET. Since there are no pendant groups on the PET chain, an initial reaction is required to generate some material which may be cross-linked. We have developed a four-step sequence for PET cross-linking, the steps are:

1. cleavage of the PET chain to produce a vinyl ester and a carboxylic acid,

2. vinyl polymerization,

3. chain-stripping to eliminate a polymeric carboxylic acid and generate unsaturation in the polymer chain, and

4. cyclization of the polyene produced above to yield cyclic cross-linked materials.

Since PET undergoes all of these reactions essentially simultaneously, it is necessary to investigate this series of reactions with a PET model, the model that we have chosen is vinyl methylterephthalate. We observe that vinyl polymerization proceeds thermally at a temperature as low as $200^{\circ} \mathrm{C}$. At this temperature the product is identical with that obtained by polymerization of the monomer by standard techniques except for the presence of a small amount of acids produced by the chainstripping process. When the thermolysis is carried out at higher temperatures, the products of the chain-stripping reaction are present in larger quantities and one may observe unsaturation of the polymer by infrared spectroscopy. At the highest temperatures that we have studied, $400^{\circ} \mathrm{C}$, cross-linking of the polymer chains to produce char is obvious by gelation experiments.

It is well-established that the initial step of PET decomposition is the formation of the vinyl ester. This material may undergo two reactions, it may be subject to further decomposition and lead to burning or this intermediate may be trapped by vinyl polymerization and lead to the cross-linking scheme noted above. This appears to be the key step in the design of a flame retardant for PET, the additive must effect the generation of the vinyl ester at as low a temperature as possible, in order to minimize decomposition, yet this must be above the processing temperature of the polymer. It is also required that this, or another, additive promote the vinyl polymerization reaction and thus feed into the above delineated scheme for PET cross-linking. Work continues in this area. 
$\left[\mathrm{CpFe}(\mathrm{CO})_{2}\right]_{2} \quad \rightleftharpoons \quad \mathrm{CpFe}^{\circ}(\mathrm{CO})_{2}$

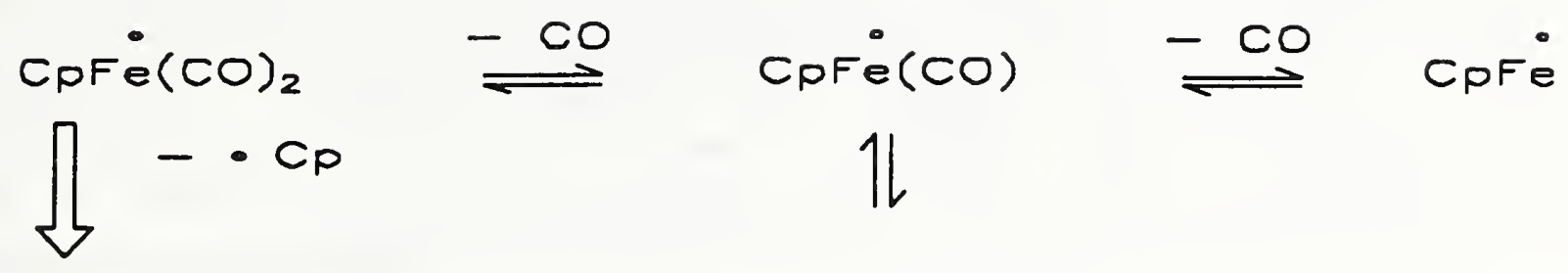

$: \mathrm{Fe}(\mathrm{CO})_{2}$

$[\mathrm{CpFe}(\mathrm{CO})]_{4}$

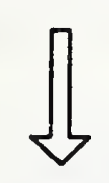

$\mathrm{Fe}+\mathrm{CO}+\mathrm{C}+\mathrm{CO}_{2}$

2 CpFe

$\mathrm{Fe}$

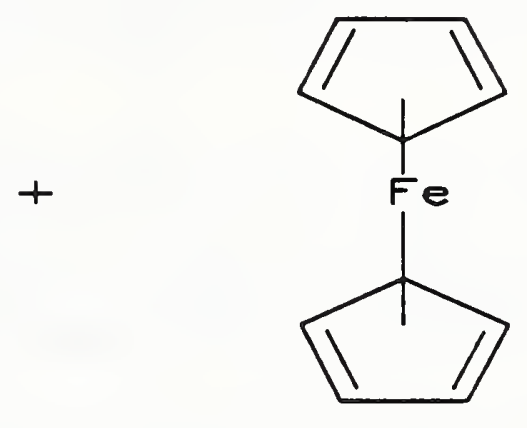

$\Longrightarrow \mathrm{HCP}+$

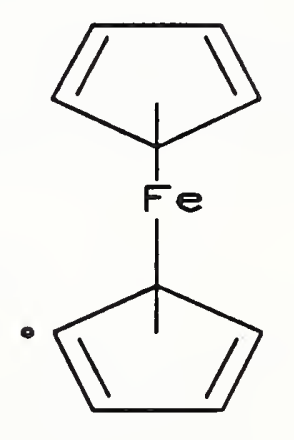

(4)

- $c_{p}+$

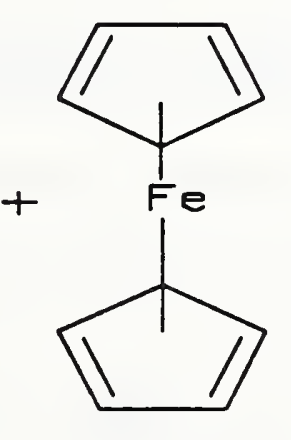

SCHEME 1

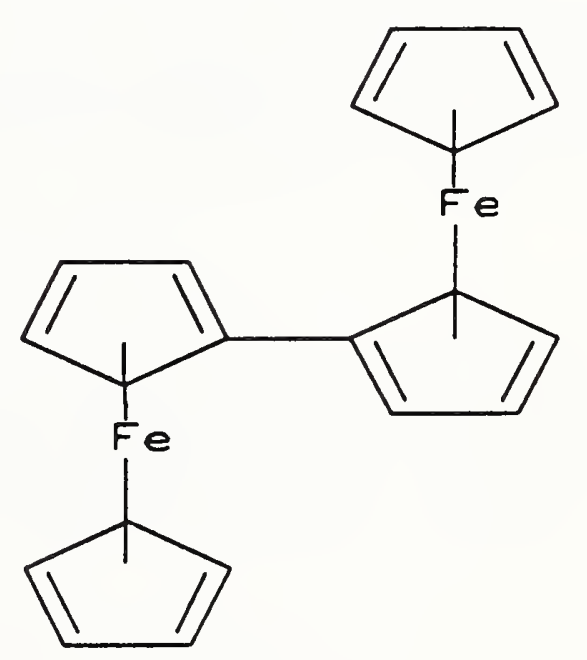


Utilization in Reducing Fire Loses. The work herein presented continues the investigation of the basic chemistry that occurs between polymers and putative flame retardants. Every step that we are able to take provides one more information in order to design an effective flame retardant for a particular polymer. The results of this work will be utilized to design additional experiments that will eventually lead to a complete understanding of the chemical reactions that are involved in flame retardation and thus permit the rational design of effective flame retardants.

\section{Reports and Papers}

1. T. Suebsaeng, C. A. Wilkie, and C. E. Brown, "Reaction of Triphenylphosphine with Poly(ethylene terephthalate) and Certain Model Compounds at Elevated Temperature," J. Polym. Sci., Polym. Chem. Ed., in press.

2. S. J. Sirdesai and C. A. Wilkie, "Wilkinson's Salt: A Flame Retardant for Poly(methyl methacrylate)," J. Appl. Polym. Sci., in press.

3. S. J. Sirdesai and C. A. Wilkie, "Mechanism of Poly(methyl methacrylate) fire retardation by Wilkinson's Salt," J. Appl. Polym. Sci., in press.

4. C. A. Wilkie and B. Huettl, "The Thermolysis of Cyclopentadienylirondicarbonyl Dimer," submitted for publication.

5. P. Chang and C. A. Wilkie, "A Mechanism for Flame Retardation of Poly(ethylene terephthalate)," manuscript in preparation. 


\section{Professional Personnel}

Marc R. Nyden, Project Leader

\section{Project Objective}

Develop techniques in the computer-aided design of materials for the purpose of achieving a better understanding of the relationships between thermal stability and the molecular chemistry of polymers.

\section{Scope}

The thermal degradation of polymers proceeds through a complex sequence of chemical reactions that frequently culminate in the formation of toxic and otherwise undesirable products. A computer model of this process would allow scientists to examine the feasibility of mechanisms proposed on the basis of limited experimental data and to explore the new degradation pathways which occur as the result of structural alterations in the model polymers. The initial studies have been directed to investigations of the kinetic and mechanistic aspects of initial events in the thermal degradation of polyethylene and related polymers.

\section{Technical Accomplishments}

1. The Distribution of Thermal Energy in the Polymer

Computer simulations of thermally induced movements in individual model polymers have demonstrated that the distribution of thermal energy is statistical. All simulations were initiated by supplying uniform distributions of atomic velocities which equilibrated to Boltzman distributions in less than 0.1 picoseconds. The heat capacities computed from these velocities were approximately equal to the statistical prediction of $5 / 2$ $N k_{B}$ and rates for the random scissions of carbon-carbon bonds in the model polymers were consistent with the predictions of statistical theories of unimolecular reactivity.

\section{Thermal Stability}

The thermal stability of the model polymers, as measured by the minimum temperature which results in at least one bond dissociation in a 10 picosecond simulation, was shown to increase with decreasing molecular weight. 
3. Mechanistic Studies

Studies of the detailed motions of model polymers have revealed pronounced coiling actions in the vicinity of bond dissociations which are consistent with proposed mechanisms for intramolecular hydrogen transfer.

\section{Reports and Publications}

"Molecular Dynamics of Initial Events in the Thermal Degradation of Polymers," Marc R. Nyden and Donald W. Noid, manuscript in preparation. 
FLAME SPREAD

\section{Professional Personnel}

James Quintiere, Project Leader

Margaret Harkleroad, Physicist

King Mon Tu, Mechanical Engineer

Robert Vettori, Engineering Technician

Steven Fischer, Post-doc

Anil Kulkarni, Guest worker

\section{Project Objective}

Develop the methodology to predict the flame spread on products and materials by using properties determined by practical test methods.

\section{$\underline{\text { Scope }}$}

This project on flame spread has concentrated its attention on vertical surfaces representative of walls. Nevertheless, the applicability of its results to other situations has been examined. For example, past work has demonstrated that results obtained under natural convection conditions for opposed flow lateral spread on a vertical surface have been applied with good accuracy to similar conditions in downward wall spread and axisymmetric horizontal spread. We have shown that the properties for lateral flame spread on a vertical wall surface can be derived from a radiant panel apparatus which we call LIFT (Lateral Ignition and Flame spread Test). Some of these properties are also applicable to other modes of flame spread such as upward spread. More recently we have been attempting to complete the identification of the general properties needed to predict upward flame spread by measuring the properties of materials and testing our ability to predict upward flame spread for these materials. The goal of this work is to establish a complete method for predicting the performance of a wall material in any compartment and environment.

\section{Technical Accomplishments}

1. Developing a Standardized Test Method for Lateral Flame Spread

Margaret Harkleroad has been leading the efforts to develop the LIFT apparatus as an ASTM standard, and in demonstrating its usefulness in the ISO TC 92 working group on flame spread. This test represents a distinct change from the tests on flammability that are based on indices of performance to a test that measures properties based on the physics of flame spread. A draft of a standard test method for the LIFT apparatus has been prepared and a first ballot has been taken by the ASTM task group, chaired by Margaret Harkleroad. This ballot yielded approximately 2 negatives out of nearly 65 votes cast, and the resolution of these results has led to improvements in the draft standard which 
will be balloted on again early this year. In ISO plans for a round robin of the LIFT procedure have been formulated, and we have been working with users of the test apparatus to insure uniformity and consistency in its use.

\section{Demonstrating the Applicability and Accuracy of LIFT}

The LIFT procedure allows the determination of several properties that would then enable the prediction of lateral or downward flame spread as a function of the local surface temperature of the material under arbitrary heating conditions. We have shown that for a number of widely different materials this has sufficient accuracy to discriminate among materials. Also, we have shown that results obtained for lateral spread in the LIFT apparatus are in agreement with the normal velocity to the flame front spreading away from the upper corner of a free standing vertical wall of PMMA, at 0.6 and $2.4 \mathrm{~m}$ high. In these experiments, measurements of the velocity of the flame front have been made along with detailed measurements of the surface temperature just ahead of the flame's heat transfer extent. The LIFT allows one to predict these results by a simple test procedure with no need to measure such temperatures. The results of these comparisons are shown (Figure 1) for PMMA at the three scales (the smallest in the LIFT), and for Mahogany taken solely in the LIFT apparatus.
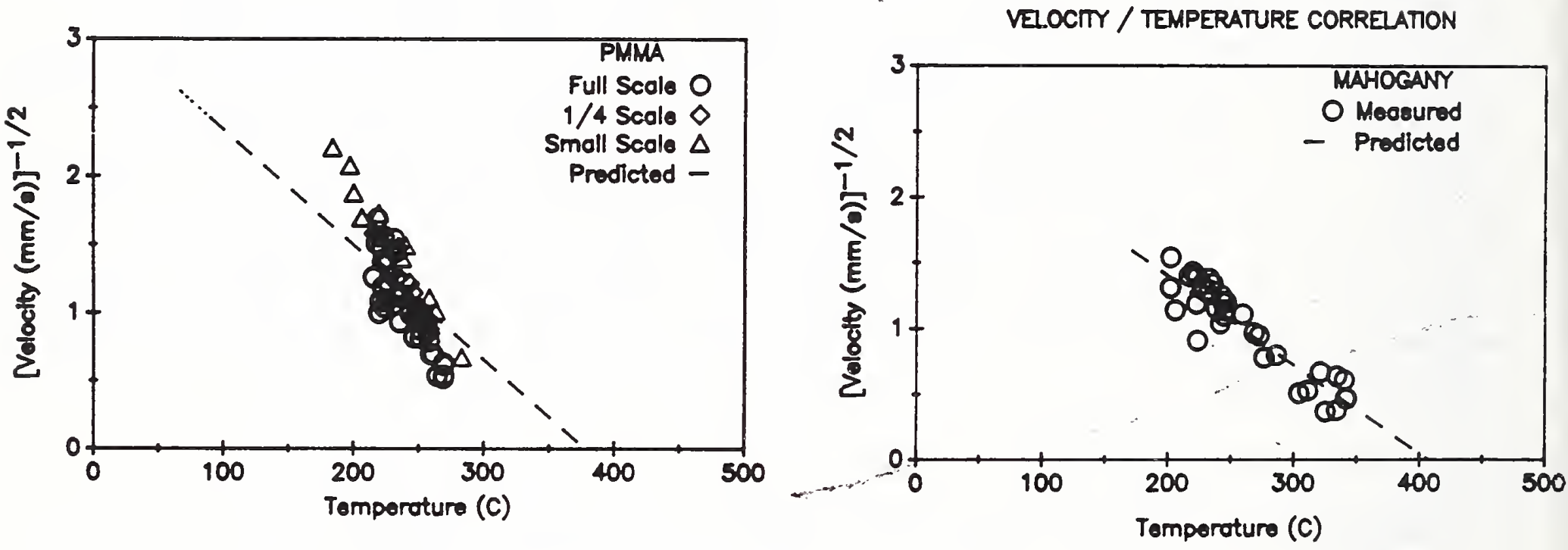

Figure 1. Applicability and accuracy of the LIFT predictions

\section{Correlating Flame Height for Burning Wall Materials}

It has been shown from theoretical analyses that the flame height for a wall fire is only a function of the energy release rate per unit width of wall burning raised to the $2 / 3$ power. Experimental results for line-burner fires against a wall give approximately this same result with the power ranging from roughly 0.6 to 0.85 . Data for unsteady wall fires were analyzed this year to investigate the applicability of this dependence of flame height on energy release rate. This 
characteristic is valuable because the height of a flame represents a distinct hazard on its own, the relationship can be inverted to use the observation to yield the energy release rate, and this relationship is needed in models proposed to predict upward wall flame spread. The flame height was measured for $0.3 \mathrm{~m}$ high PMMA and wood particle board burning under a range of radiant heating exposures. The energy release rates were determined from the instantaneous mass loss rates of the samples. These transient data for the various conditions and both materials are plotted in Figure 2. The results are within 25\% of theory and line-burner data which serve to give confidence in the use of this correlation to the modeling of the dynamic process of flame spread.

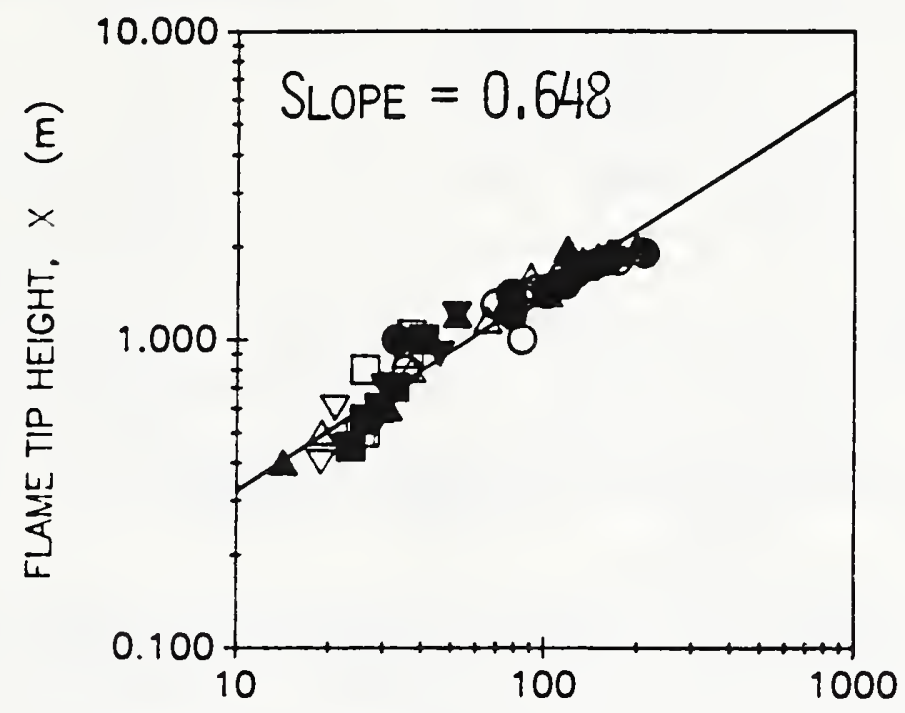

HEAT RELEASE RATE PER UNIT SAMPLE WIDTH, Q $(\mathrm{KW} / \mathrm{m})$

Figure 2. Flame height for transient wall fire of wood and PMMA

\section{Predicting Upward Flame Spread}

We have begun to evaluate our ability to predict upward flame spread on particular wall construction materials. This has been done through a modeling strategy, collecting the needed material fire property data for the model, and conducting tests on upward flame spread on samples 1.2 and 2.4 high. The results have been promising in two respects: (1) all of the data can be developed that is needed for the model (some comes from the LIFT apparatus), and (2) initial results for previous experiments on wood particle board were able to predict the extent of spread for various ignition flames. These results show that the model is capable of predicting the nonsustained spread case as well as the rate of spread in the sustained case. This is very important to the evaluation of a product's performance since it can reveal under what conditions sustained spread is possible. In a previous study we found that it is necessary to preheat this particle board to permit sustained upward spread. It required sufficient preheating at 0.42 $\mathrm{W} / \mathrm{cm} 2$ or approximately a minimum surface temperature of $100^{\circ} \mathrm{C}$. The predicted results for the particle board are shown in Figure 3 for two values of $\mathrm{K}$, a coefficient in the formula for flame height. The lower value considers a fixed flame heat flux over the continuous luminous flame height, and the higher value corresponds to the flame tip. A unique feature of this approach has been to derive a transient mass loss rate for the material directly from a small scale experiment that 
has been designed to simulate the flame heating conditions for the early part of the process. This has to be generalized to include a flame heat transfer model that includes the radiation component indicative of the material and size effect, and to establish a method for interpreting material calorimetry tests, such as the Cone Calorimeter, to permit the prediction of the transient mass burning rate of the particular material.

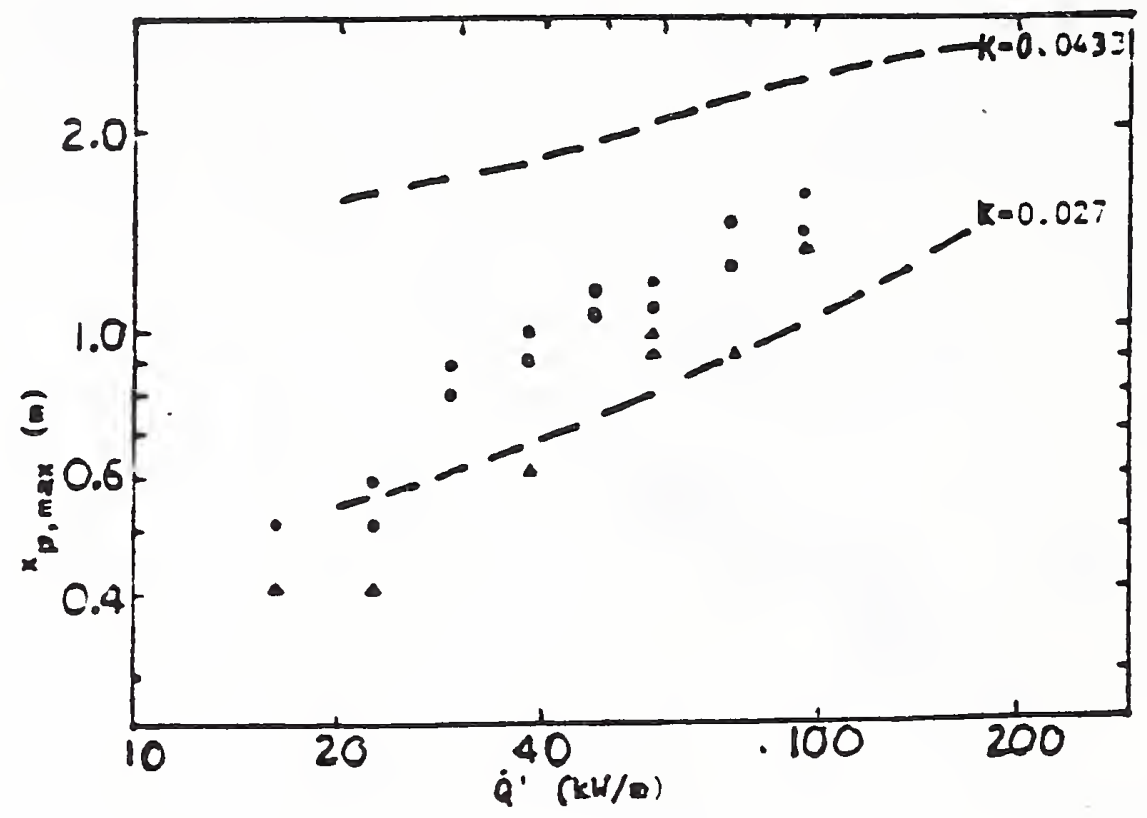

Figure 3. The maximum distance spread on wood for a range of igniting flame energy release rates, experimental results and theory.

\section{Reports and Publications}

"Upward Turbulent Flame Spread on Wood Under External Radiation", S. Saito, F. A. Williams, I. S. Wichman, and J. G. Quintiere, ASME Paper No. 87-HT-56, NTHC, Pittsburgh, PA, August 9-12, 1987 (to be published J. Heat Transfer)

"The Application of Flame Spread Theory to Predict Material Performance", J. G. Quintiere, J. of Research of the National Bureau of Standards, 93, 1, Jan - Feb, 1988.

"Ignition and Flame Spread Measurements of Aircraft Lining Materials", M. Harkleroad, Nat. Bur. Stand., NBSIR 88-3773, May 1988.

"State of Fire Research and Safety", J. G. Quintiere, Second Proceedings of the Fire Safety Science International Symposium", Tokyo, June $13-17,1988$.

\section{Related Grants}

"Prediction of Fire Dynamics", J. de Ris, R. L. Alpert, Factory Mutual Research Corp.

"Fire Propagation on Concurrent Flows", A. C. Fernandez Pello, U. of Cal. (Berkeley).

"Upward Flame Spread on a Vertical Wall", A. Kulkarni, Penn. St. Univ. "Wind-Aided Flame Spread", F. Fendell, TRW Space and Technology Group. 
CENTER FOR FIRE RESEARCH

NATIONAL BUREAU OF STANDARDS

FY 88

Institution: Factory Mutual Research Corporation

Grant No.: 60NANB5D0560

Grant Title: Prediction of Fire Dynamics

Principal Investigators:

John de Ris and Ronald L. Alpert

Other Professional Personnel:

G.H. Markstein

M.A. Delichatsios

L. Orloff

H.W. Emmons

NBS Scientific Officer:

Dr. Henri Mitler

Technical Abstract:

This work is divided into four tasks, each of which is designed to provide essential inputs for comprehensive models of burning and fire growth. Such predictive models will reduce fire losses through the development of sciencebased techniques for rating material flammability.

Task 1. Prediction of Fires in Buildings (H.W. Emmons)

Please see a separate summary under this title.

Task 2. Fire Radiation and Toxi c Product Release - An objective of the research conducted over the past year was to develop correlations that predict the radiation from buoyant turbulent jet flames for a wide range of fuels in terms of the respective fuel smoke point, chemical bonding structure, and the ambient oxygen concentration. This is an ongoing study in which our results will be extended into simulations of turbulent wall fires and the prediction of incomplete combustion product generation, particularly those that are corrosive or toxic. The correlations developed in this study will provide essential inputs on flame radiation for fire growth predictions.

The experiments were performed inside a $1.2 \mathrm{~m}$ dia water-cooled enclosure which allowed us to independently control the adiabatic stoichiometris flame temperature and stoichiometric mass ratio by adding: 1) either oxygen or nitrogen to the supplied ambient air, and 2) nitrogen to the supplied fuel. Measurements of the radiant fraction (of the theoretical heat release rate) were obtained with a wide angle radiometer with parallax corrections derived from a traversing horizontal slit radiometer. The burner nozzles were gold plated to minimize radiative emission from their hot surfaces. Considerable testing of various experimental conditions was performed to insure reproducibility and to minimize dependence on the specific experimental conditions.

For the measured $6-20 \mathrm{~kW}$ fires the radiant fractions were effectively independent of the: 1) overall heat release rate, 2) fuel-jet orifice diameter, 3) degree of oxidant overventilation, and 4) fuel-mixture supply temperature. Measurements were made over a very wide range of stoichiometric mass ratios, $S$, ranging from 2 to 20 , and adiabatic flame temperatures, $T_{a d}$, ranging from 
$1900 \mathrm{~K}$ to $2600 \mathrm{~K}$. For typical values of $S \geq 12$, the radiant fractions were independent of $S$ and all the fuels had a similar dependence of radiant fraction, $x_{R i}$, on $T_{\text {ad }}$ described by the formula:

$$
x_{R i}=\frac{\left(T_{a d}-T_{b}\right) x_{R i}^{*}}{\left(T_{a d}^{*}-T_{b}\right)+x_{R i}^{*}\left(T_{a d}-T_{a d}^{*}\right)}
$$

where $x_{\mathrm{R} i}^{*}$ is the reference value of $x_{\mathrm{Ri}}$ at "standard conditions" [ $\mathrm{T}_{\mathrm{ad}}^{*}=2200 \mathrm{~K}$ and $\left.S^{*} \stackrel{\mathrm{R} i}{=} 15\right]$, and $\mathrm{T}_{\mathrm{b}} \cdot=1675 \mathrm{~K}$ was a good empirical fit for all measured fuels $\left(\mathrm{CH}_{4}, \mathrm{C}_{2} \mathrm{H}_{2}, \mathrm{C}_{2} \mathrm{H}_{4}, \mathrm{C}_{2} \mathrm{H}_{6}, \mathrm{C}_{3} \mathrm{H}_{6}, \mathrm{C}_{3} \mathrm{H}_{8}, \mathrm{C}_{4} \mathrm{H}_{8}\right)$. The values of $x_{\mathrm{R}}^{*}$ were ranged in agreement with the independently measured classical laminar smoke-point values at the identical "standard condition".

For smaller values of $S<12$ the radiant fractions decreased with decreasing $\mathrm{S}$. The amount of decrease was considerably greater for the alkenes $\left(\mathrm{C}_{2} \mathrm{H}_{4}\right.$, $\mathrm{C}_{3} \mathrm{H}_{6}$ and $\left.\mathrm{C}_{4} \mathrm{H}_{8}\right)$ and alkyne $\left(\mathrm{C}_{2} \mathrm{H}_{2}\right)$ than for the alkanes $\left(\mathrm{CH}_{4}, \mathrm{C}_{2} \mathrm{H}_{6}\right.$ and $\left.\mathrm{C}_{3} \mathrm{H}_{8}\right)$. Thus for $S<12$, there is some additional effect of chemical structure on $x_{R}$ beyond that included in the classical smoke point. We are currently measuring the radiant fractions for more fuels to examine this more complicated dependence on the chemical structure of the fuels.

Publications

1. Orloff, L., de Ris, J., Delichatsios, M.A.: "Radiation from Buoyant Turbulent Diffusion Flames," Poster Session Paper P22-156, 22nd International Symposium on Combustion, Seattle, WA, Aug. 14-19, 1988.

Task 3. Wall Fire Flame Radiation - The objectives are extensions of previously established relationships for fire radiation to the case of vertical walls burning, comparison of the results with existing analyses of wall burning, and development of improved models of wall burning based on experimental findings. As in other related work the significance of studies of fire radiation rests on the fact that in many fires energy transfer from the flame to the fuel and to the surroundings occurs predominantly by thermal radiation. The rate of fire growth and the spread of fire to new fuel elements depends critically on this radiant energy transfer, and its quantitative assessment is thus essential for predicting fire behavior.

In the present task, wall fires of solid fuels are simulated by burning gaseous hydrocarbon fuels on a water-cooled vertical porous metal surface under steady-state conditions. The burner is subdivided into a number of panels of equal height, so that the simulated pyrolysis height can be varied by the choice of the number of fuel-supplying panels. In addition, the limiting case of zero pyrolysis height can be studied by supplying the fuel from a slot burner at the base of the wall.

Fuel is supplied to the burner by electronic flow controllers, and the heat transferred to each panel is determined from the temperature rise of the cooling water, measured by differential thermocouples. The total radiant emission from the fire is measured with a wide-view-angle radiometer employing a spectrally flat thermopile sensor. In addition, the vertical distribution 
of radiant power per unit height emitted by narrow horizontal slices across the width of the fire is measured with a scanning slit radiometer. The scan along the height of the fire is obtained by the use of an electromagnetically deflected plane front surface mirror operated in a linear ramp mode. A fastresponse spectrally flat pyroelectric sensor is used in this instrument. A new version of this instrument, improved by the use of spherical-mirror optics, has been recently evaluated, and the expected enhanced signal-to-noise ratio and vertical resolution has been confirmed.

Initial work under this task was performed with a previously developed burner consisting of six 380-mm wide and 220-mm high sintered-metal panels topped by a 520-mm high metal plate. Since the fine-grained sintered metal of the burner was susceptible to clogging by soot, the work was restricted to ethane, which produces relatively small amounts of soot. A new burner incorporating various design improvements has been assembled, and work with this burner is about to begin. One important new feature is the use of an outer layer of coarse-grained sintered metal that is expected to prevent permanent clogging by soot, so that future work can be extended to fuels of greater sooting tendency than ethane. The height of the individual panels has been reduced to $127 \mathrm{~mm}$ to provide improved resolution of the simulated pyrolysis height. As another new feature, heat transfer instrumentation has been incorporated in the metal plate above the burner panels.

Although the data obtained previously with the old burner are of a preliminary nature, they nevertheless provided valuable results which have recently been examined in greater detail. In this recent work, 1 correlations have been established between the radiative fraction and the convective $B$-number, and between normalized values of radiant flux and height. These relationships and their significance are being further investigated and it is planned to extend the correlations to the data to be obtained with the new burner. The results will also be compared with existing theoretical models of wall-fire combustion and will also be used to extend correlations between flame radiation and fuel smoke points to wall fires.

Concurrently with the study of wall fires, measurements of smoke points and of radiant emission from laminar diffusion flames have been performed. This continuing work, which is also partly supported by the Gas Research Institute, is of interest for the wall-fire study in connection with correlations between flame radiation and smoke points and is also of importance for the study of turbulent flames under Task 2. In recent work on laminar flames, 2,3 a new method for smoke-point determination based on measurement of radiant extinction by soot in the combustion products has been developed to replace the customary visual observation. The results of this work led to general correlations for the smoke point and for the radiant emission, which will be of value in the formulation of models for soot formation and radiant emission, both of laminar flames and of turbulent flame jets and wall fires.

Publications

1. de Ris, J., "Radiation from Diffusion Flames - Smoke Point," presented at the 9th Joint Panel Meeting of the UJNR Panel on Fire Research and Safety, May 4-8, 1987. 
2. Markstein, G.H., "Correlation for Smoke Points and Radiant Emission of Laminar Diffusion Flames," presented at the Spring Technical Meeting, Central States Section, The Combustion Institute, Argonne, IL, May 11-12, 1987 .

3. Markstein, G.H., "Correlations for Smoke Points and Radiant Emission of Laminar Hydrocarbon Diffusion Flames," to be presented at the 22nd International Symposium on Combustion, Seattle, Washington, Aug 14-19, 1988.

Task 4. Transient Pyrolysis and Flame Radiation from Charring Fuels - Measurements needed to handle the vertical fire growth problem include material pyrolysis rates (including charring materials) and flame radiation characteristics which are being obtained in the new flammability apparatus in the Basio Research Department of FMRC. Coupled with models for a) gaseous burning (developed for pool fires and walls at FMRC), b) heat transfer, c) pyrolysis rates, and d) flame spread, already developed as a part of this program, the measured flammability properties will lead to predicting the occurrence and the development of sustained flame spread on a charring, composite or laminated fuel.

The following results have been achieved during the previous reporting period:

1) The new material flammability apparatus has been successfully used to obtain pyrolysis rates for charring materials (results for two different particle boards, Douglas fir wood and PMMA, as a baseline measurement, are contained in Reference 2). Preliminary analysis of the data shows that the pyrolysis behavior of charring materials is consistent with semi-analytical models developed earlier at the Basic Research Department of FMRC.

2) An outline for a comprehensive upward flame spread model ${ }^{1}$ has been assembled. Moreover, a simplified reduction of the model has allowed identification of the parameters controlling flame spread. These parameters are:

$$
\begin{aligned}
& \chi_{R} \\
& \chi_{R} \frac{\Delta H_{c}}{\Delta H_{v}} \\
& \frac{k_{w}^{2} \pi\left(T_{p}-T_{s}\right)^{2}}{\alpha_{w}} \\
& \dot{Q}_{c h}^{\prime} \\
& \gamma
\end{aligned}
$$

: radiant fraction from the wall fire

: a parameter proportional to the ratio of flame height to pyrolysis length.

: a characteristic ignition parameter

: the chemical heat release rate from the fire

: ratio of any external heat flux to the flame radiation heat flux

Notice that the present program addresses the characterization of $\chi_{R}$ and $\dot{Q}_{c}$ ch (through the pyrolysis rate and heat of vaporization). 
A significant achievement during the previous reporting period was undoubtedly the successful operation of the new material flammability apparatus. over fifty tests ${ }^{2}$ have been conducted with the assistance of Prof. K. Saito for four materials (2 particle boards, Douglas fir wood, and PMMA) and heat fluxes ranging from 2 to $16 \mathrm{~W} / \mathrm{cm}^{2}$, which is a unique range of operation. In addition to the weight loss, temperatures at four locations inside the samples $(12 \mathrm{~cm}$ in diameter, 1 or $2 \mathrm{~cm}$ thick) were measured. Flame radiation was not measured during these tests because no steady buoyant flame-jet was established. For this reason the exit nozzle has been recently modified by using a conical diffuser with an exit nozzle diameter of 1 in., large enough to allow the establishment of a turbulent buoyant flame. In addition, provisions have been made to purge the quartz plate by using methane fuel instead of heated nitrogen through the annular sand box distributor (see Fig. 1). We have found in preliminary experiments that a purge by methane readily stabilizes the turbulent buoyant flame of the pyrolyzing gases on the exit nozzle's perimeter. We plan to unravel the effects of methane on the flame radiation of the pyrolyzing gases by measuring the radiation from turbulent flames of gaseous mixtures of methane and other gaseous fuels (e.g. propane, acetylene) whose radiation properties are known. Such measurements can be made in the material flammability apparatus by introducing fuel gases in place of a charring sample.

After we completed the experiments with the particle board sample, we found out that its density was not uniform: the density was thirty percent less at its center than near its top or bottom surface. To allow a direct comparison with theory, we plan to use a uniform charring fuel stock for additional tests by manufacturing, probably, our own cellulosic board. The assembled data are being analyzed, but it is worth sharing some of the results of a preliminary analysis:

1) By conducting tests with carbon blackened and pure particle boards, it was shown that spectral absorption selectivity affects the "ignition" time, but not the ensuing pyrolysis history;

2) It is encouraging that the measured pyrolysis histories are consistent with our semi-analytical model for the pyrolysis of charring and noncharring materials.

3) The time to "ignition" (i.e. copious pyrolysis) is controlled by surface reradiation losses, which are not properly accounted for in existing analytical models and methodologies. A new semi-analytical model has been developed to account for this important phenomenon. Our recent theoretical analysis and experimental results have indicated that in depth radiation absorption in "black" PMMA significantly affects the "ignition" or pyrolysis time. We have developed a procedure to uniformly cover the surface of all samples with a black paint which would absorb all radiation at the surface. Such a procedure will render results which would be easy to interpret because "ignition" times would depend only on conduction losses into the solid (plus surface reradiation, of course).

Our immediate plans include: a) analyze the numerous experimental pyrolysis data with particle board; b) repeat experiments with black PMMA covered 
with a thin layer of black paint; c) use cone calorimeter to document spectral radiation effects (in comparison with quartz heaters); and d) measure flame radiation from the burning of pyrolyzing gases.

Publications

1. Delichatsios, M.A., "An Outline for a Comprehensive Model for the Burning and Upward Flame Spread on Charring Materials in Wall Fires," Eastern Section Meeting of the Combustion Institute, NBS, November 1987.

2. Saito, K., et al., "Transient Pyrolysis Tests of Charring Material under External Radiation up to $16 \mathrm{~W} / \mathrm{cm}^{2}$, "Central Section Meeting of the Combustion Institute, Indianapolis, May 1988. 


\section{CENTER FOR FIRE RESEARCH \\ NATIONAL BUREAU OF STANDARDS \\ FY 88}

Institution: Factory Mutual Research Corporation

Grant No: 60NANB5D0560

Grant Title: Prediction of Fire Dynamics -- Task 1. Prediction of Fires in Buildings

Principal Investigator: $\quad$ Prof. Howard W. Emmons, Retired

Division of Applied Sciences

Harvard University

Cambridge, MA 02138

NBS Scientific Officer: $\quad$ Dr. Henri Mitler

Technical Abstract:

Introduction The computer Fire Model for calculation of the growth of fire in an enclosure has been under development at NBS for several years. This project has contributed to this effort as follows:

1. Improve the user-friendly nature of the latest update program "FIRST."

2. $\quad$ Examine a range of cases to find and remove "bugs."

3. To improve the required heat transfer relations for a ceiling jet.

1. The user friendly input. The input at user's choice can be run through completely or input specific data introduced through a menu. Many minor improvements have been made such as the use of the notation ( $\mathrm{Y} / \mathrm{N}]$ ) which answers a question with either caps or lower case letters and for which a simple <Return> selects the commonest response [].

2. Over 110 specific cases have been run. These runs examined the effect of many variables, e.g., size of room, size of vent, height of fuel, height of vent, location of fuel. A few of these results are presented in a paper at the second meeting of the International Association of Fire Safety Science. The most important effect on fire growth are those changes that affect the availability of oxygen.

During these runs, 18 specific problems were found. A list of all these needed fixes has been given to NBS for future incorporation into FIRST. These problems included items such as permit user to add or remove objects through the menu, there is a fraction of "hydrocarbons," $f_{(\mathrm{HC})}$, listed in the output but not in the input, sometimes a burning object is starved of $\mathrm{O}_{2}$ and unstarved every two seconds, if the user asks for a nonexistent object or vent, the program should not bomb but should return to the menu so the user can fix it, etc. (The full list is available on request.)

This series of runs also disclosed a long list (38) of specific needed permanent (or user selectable) phenomena additions. This list included such items as burning in the hot layer, wall burning (now being implemented at NBS), a variable property fire (e.g., initial fire spread and later char burning), sprinkler response and extinguishment, etc. (The full list is available on request.) 
The most severe "bug" is, of course, the failure of the program to complete a run because of "Divide by Zero," "Underflow," "Overflow," or failure to converge at the smallest time step. Time did not permit the clarification and permanent removal of most of these problems but three types of "cures" were often effective.

a. Set a smaller tolerance. This gave greater accuracy of a converged solution and sometimes the next time step came easier.

b. Relax the tolerance. Even a very poor tolerance as bad as 0.1 (instead of $10^{-5}$ ) gave answers to two percent except in critical regions (immediately after target ignition) where errors might be $20 \%$ (satisfactory for many practical fire purposes).

c. Make some small (1\%) change in heat of pyrolysis, heat of combustion, or move an object. This can remove an accidental coincidence of difficulties such as "Divide by Zero."

Various test results, furniture calorimeter data, etc., should be made available to FIRST via the following observations. An object or objects under test that are to be represented as a single object in FIRST should have properties determined by:

1. $\tau, k, \rho, c, T_{i g}$, set for item quickest to ignite (as for example a cellular plastic cushion).

2. $\varepsilon$ set high $(\sim 0.98)$ because char is generally carbon and is black.

3. $\mathrm{M}$ for each component set at fraction you expect to actually burn (there is often a lot of char unburned).

4. The initial burning radius depends on the size of the ignition source (see figure).

5. The fire spread parameter A depends upon the spread mechanism and is generally somewhat larger than the mass

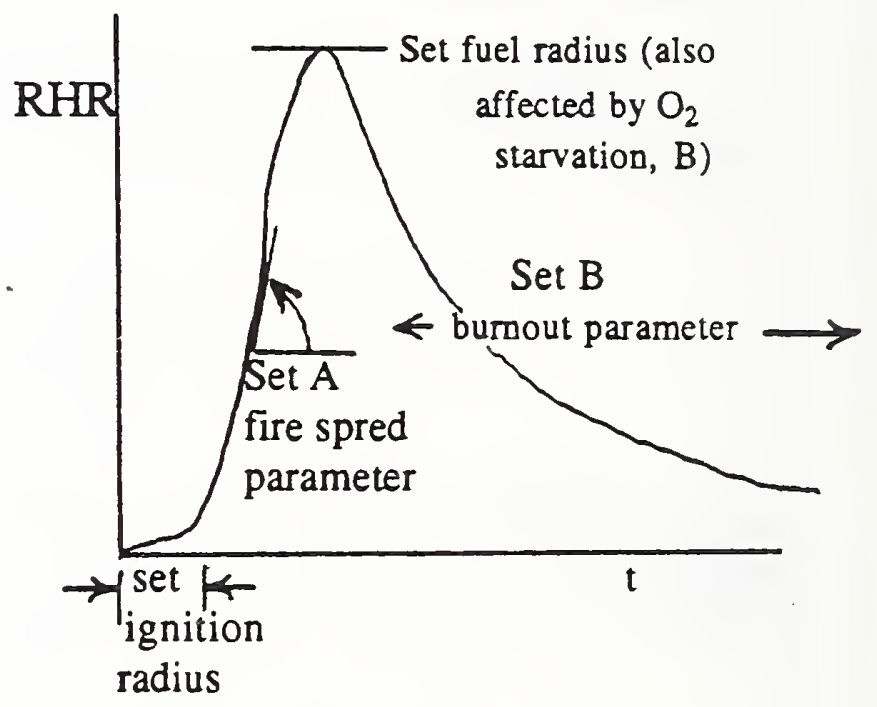
weighted average of component A's. It sets the slope of the rapid rise (see Figure).

6. The effective fuel area sets the maximum rate of heat release (it approximates the fuel projected area).

7. The burnout parameter B sets the time of char burnout.

8. All other parameters are the mass weighted average.

3. Ceiling jet heat transfer. When hot gas, without combustion, at temperature $T_{s}$ (initial temperature $T_{0}$ ) flows at constant rate $u$ along a ceiling at initial temperature $T_{w}$, it transfers heat to the ceiling at rate $h\left(T_{s}-T_{w}\right)$ and also loses heat to the ambient at the rate $h_{a}\left(T_{s}-T_{a}\right)$. The wall, too, loses heat to the ambient at the rate $h_{w a}\left(T_{w}-T_{a}\right)$. Thus, the ceiling jet is cooled while the ceiling is heated. If a corridor were very long, both the ceiling jet and ceiling would have temperature $T_{a}$ at large distances. 
The solution to this heat transfer process is:

$$
\begin{aligned}
& \theta_{s}(\chi, \tau)=e^{-\left(P_{s}+P_{2}\right) \chi}\left\{e^{-\left(P_{w}+P_{w 2}\right)(\tau-\chi)} I_{0}\left(2 \sqrt{P_{w} P_{s} \chi(\tau-\chi)}\right)\right. \\
& \left.+\left(P_{w}+P_{w a}\right) \int_{0}^{\tau-\chi} e^{-\left(P_{w}+P_{w 2}\right) t} I_{0}\left(2 \sqrt{P_{w} P_{s} \chi t}\right) d t\right\} u(\tau-\chi) \\
& \theta_{w}(\chi, \tau)=\left(P_{w}+P_{w a}\right) e^{-\left(P_{s}+P_{a}\right) \chi} \int_{0}^{\tau-\chi} e^{-\left(P_{w}+P_{w a}\right) t} I_{d}\left(2 \sqrt{P_{w} P_{s} \chi t}\right) d t u(\tau-\chi) \\
& \theta_{s}-\theta_{w}=e^{-\left(P_{s}+P_{a}\right) \chi} e^{-\left(P_{w}+P_{w a}\right)(\tau-\chi)} I_{0}\left(2 \sqrt{P_{s} P_{w} \chi(\tau-\chi)}\right) u(\tau-\chi)
\end{aligned}
$$

where $\theta_{s}=\frac{T_{s}-T_{a}}{T_{0}-T_{a}}, \theta_{w}=\frac{T_{w}-T_{a}}{T_{0}-T_{a}}, \chi=x / L, \tau=t u / L$,

$$
u(\tau-\chi)= \begin{cases}0 & (\tau<\chi) \\ 1 & (\tau>\chi)\end{cases}
$$

$P_{s}=\frac{h L}{\rho_{s} u \delta_{s} C p_{s}}, P_{w}=\frac{h L}{\rho_{w} u \delta_{w} C p_{w}}, P_{a}=\frac{h_{a} L}{\rho_{s} u \delta_{s} C p_{s}}, P_{w a}=\frac{h_{w a} L}{\rho_{w} u \delta_{w} C p_{w}}$,

$I_{0}(z)$ is the modified Bessel function of the first kind. $\chi$ is the fractional length. $\tau$ is the fractional location of the leading edge of the ceiling jet. If the ceiling jet has not reached the point of interest $(\tau<\chi), \theta_{s}=\theta_{w}=0$, i.e., the jet and wall are both at ambient temperature.

While these formulas appear complex, they can be and will be precalculated and made available as simple algebraic expressions. 
Institution: University of California, Berkeley

Grant No: 60NANB7D0737

Grant Title: Fire Propagation in Concurrent Flows

Principal Investigator: Professor A.C. Fernandez-Pello

Department of Mechanical Engineering University of California

Berkeley, CA 94720

Other Professional Personnel: B. Amos, Doctoral Graduate Student

L. Zhou, Doctoral Graduate Student

NBS Scientific Officer: Dr. H.R. Baum

\section{Technical Abstract:}

A research program is being conducted to study the mechanisms controlling the spread of flames over the surface of a solid combustible in an oxidizing gas flow. The objective of the research is to provide fundamental information about the spread of fire for its use in the development of models of fire growth in rooms, and of test methods of the flammability of materials.

Research performed during this reporting period includes two theoretical studies (one on ignition and the other on flow assisted flame spread) and an experimental study of turbulent flame spread. The ignition work consists of a numerical analysis of the ignition and flame establishment over a combustible solid that is vaporizing in a stagnation point air flow due to an externally applied irradiance. The flame spread work is a numerical model of the spread of flames over a thin charring combustible in a gas flow moving in the direction of spread. The experimental work is a study of the effect of flow turbulence on the rate of opposed flow flame spread.

\section{Stagnation Flow Ignition of a Vaporizing Surface}

In this work, an analysis is developed of the ignition of a vaporizing flat combustible solid and of the subsequent establishment of a diffusion flame over it, in a stagnation point oxidizing gas flow. The gasification of the solid is produced by an external radiant flux applied perpendicular to the surface.

The vapor ignition process is modeled as follows. A planar combustible solid at its vaporization temperature is in a stagnation point air flow at ambient temperature. At the initial time the solid surface is suddenly subjected to a constant radiant flux perpendicular to the surface. The solid absorbs the radiation at its surface and immediately begins to vaporize, mixing with the ambient air. The vaporized fuel absorbs some of the incoming radiation and eventually becomes hot enough to initiate the ignition of the fuel/air mix- 
ture. After the onset of ignition, the external radiation is removed and the chemical reaction is let to evolve until it becomes a self-sustained, steady, diffusion flame. In the analysis it is assumed that the adsorption of radiation by the gas is independent of the temperature, but that is directly proportional to the fuel concentration. The combustion of the mixture is modeled using a one step chemical reaction of the Arrhenius type.

The problem governing equations are solved numerically using an implicit method except for the temperature in the reaction rate term which is handled explicitly. A movable grid is used to track the propagation of the flame after ignition. The predicted evolution of the temperature and species profiles shows the existence of three regimes. The first regime corresponds to the period from the instant when the external irradiance is first applied to the onset of ignition. The evaporation of the fuel causes the generation of two opposing stagnation point flows, one of fuel and the other of oxidizer, at whose interface the fuel and oxidizer mix by diffusion. During this period the fuel vapor absorbs radiation and heats up eventually causing the ignition of the mixture. After ignition, the combustion reaction propagates through the mixture away from the solid surface. This premixed propagation process leads to the generation of a diffusion flame once all the fuel ahead of it is consumed. The diffusion flame adjusts its position until finally it reaches its steady state position near the wall. A characteristic example of the predicted evolution of the temperature distribution is shown in Fig. 1 .

\section{Model of Concurrent Flame Spread Over a Thin Charring Solid}

A theoretical model is developed of the spread of a flame over the surface of a thin combustible solid in a gas flow moving in the direction of flame propagation. The combustion reaction and the solid pyrolysis are modeled respectively with a one-step, second order, and with a zero order Arrhenius rate. The charring of the solid is modeled by treating the solid as a two component material, the combustible component that gasifies, and the char that remains occupying the initial solid volume. To simplify the mathematical solution of the problem, a Hagen-Poiseuille flow is prescribed, thus eliminating the need for using the momentum conservation equations. The transient, reactive, gas phase balance equations of energy and species coupled at the interface to the solid phase balance equations are solved numerically to describe the evolution of the flame and solid properties, and through them to predict the variation with time of the flame, pyrolysis and burn-out distances and their dependence on the flow velocity.

The model is applied to the description of the spread of flames over paper sheets in a concurrent air flow. In Fig. 2 the predicted variation with time of the flame, pyrolysis and burn out distances are presented for several flow velocities. The experimental data of Loh and Fernandez-Pello (1st Int. Symp. Fire Saf. Sci., 65, 1986) are included for comparison. It is seen that the predictions of the model agree well with the experiments. Particularly important is the description of the transition of the flame spread process from an initial accelerative stage to a final steady state. The transition is determined by the interaction between the progress of the pyrolysis and the burn out fronts, and their effects on the flame length. The model also predicts a flame extinction limit due to increased concurrent velocity that is very close to the experimentally determined value. 
Experiments are currently underway to study the effect of flow turbulence on the rate of flame spread over a solid combustible. The experiments are carried out in a combustion tunnel facility that has been described in a previous report. The turbulence intensity in the tunnel test section is varied by means of grids of different sizes placed at the exit of the tunnel converging nozzle. The velocity and turbulence intensity of the flow field are measured with a Laser Doppler Velocimeter. The flame spread rate is obtained from the temperature history of thermocouples placed at fixed distances along the combustible surface.

Currently we are performing experiments to measure the rate of flame spread over the surface of thick (1/2") PMMA sheets as a function of the velocity and turbulence intensity of an air flow opposing the direction of flame spread. The opposed flow mode of flame spread was selected to do the initial experiments because, it being easier to measure accurately the spread rate, it permits better familiarization with the experimental technique. Once this initial study is completed, the study of the effect of turbulence on the concurrent mode of flame spread will follow.

Preliminary results of the measurements of the opposed flow flame spread rate over PMMA as a function of the flow velocity for turbulent intensities of $1 \%, 15 \%$ and $20 \%$ are presented in Fig. 3. The results, although preliminary, show that for a given flow velocity the spread rate decreases moderately as the turbulence intensity increases. This result is explained by the fact that as the flow turbulence intensity increases the transfer of heat upstream from the flame front decreases, and consequently, so does the flame spread rate.

$\underline{\text { Reports and Papers }}$

1. Kodama, H., Miyasaka, K. and Fernandez-Pello, A.C., "Extinction and Stabilization of a Diffusion Flame on a Flat Combustible Surface with Emphasis on Thermal Controlling Mechanisms," Combustion, Science and Technology, 94, 1-6, pp. 37-50 (1987).

2. Amos, B., Kodama, H. and Fernandez-Pello, A.C., "An Analysis of the Ignition by Vapor Radiation Absorption of Vaporizing Fuel at Zero Gravity," presented at the AIAA 25th Aero. Sci. Meeting, Reno, Nevada, 1987. Also Progress in Aeronautics and Astronautics (in press), 1988.

3. Amos, B., "Ignition and Flame Propagation Studies Over a Flat Fuel

Surface," Ph.D. Thesis, Department of Mechanical Engineering, University of California, Berkeley, August 1987.

4. Di Blasi, C., Cresticelli, S., Russo, G. and Fernandez-Pello, A.C., "Predictions of the Dependence on the Opposed Flow Characteristics of the Flame Spread Rate Over Thick Solid Fuels," 2nd Int. Symp. of Fire Safety Sci., (accepted) Japan, June 1988.

5. Di Blasi, C., Cresticelli, S., Russo, G. and Fernandez-Pello, A.C., "Model of the Flow Assisted Spread of Flames Over a Thin Charring Combustible," accepted for presentation at the 22nd Symposium (Int.) in Combustion, Seattle, WA, August 1988 . 


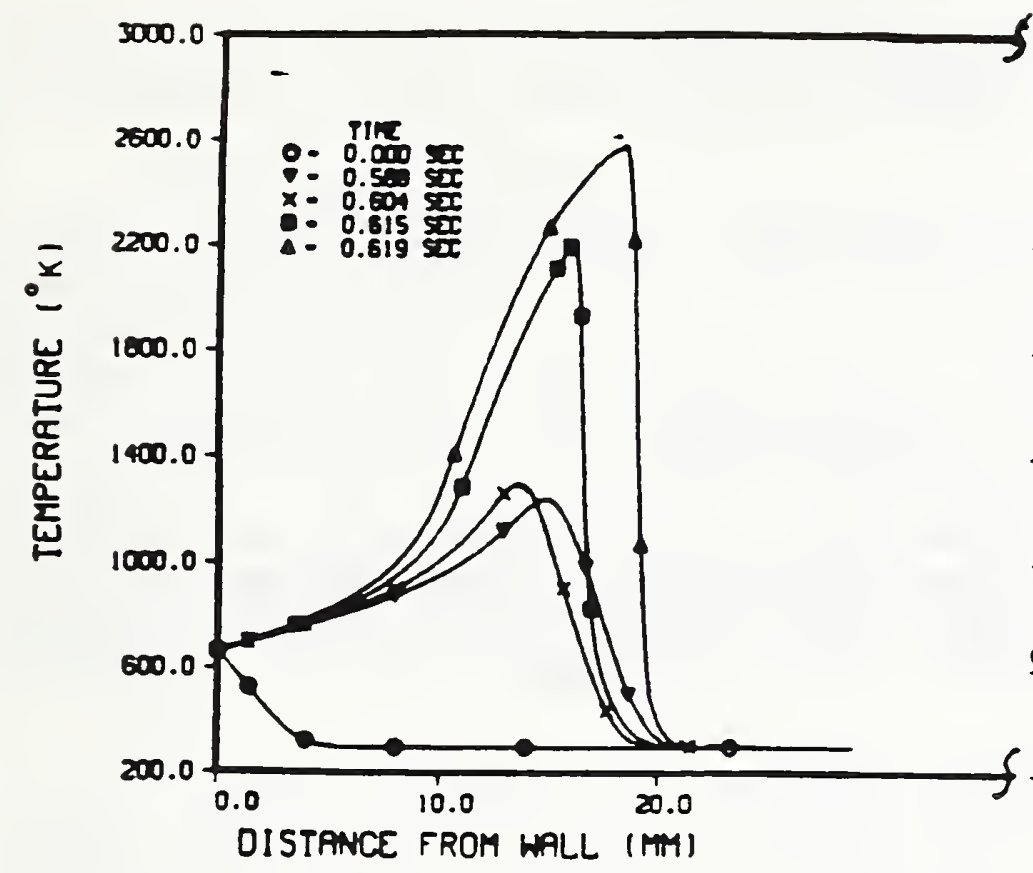

(a)

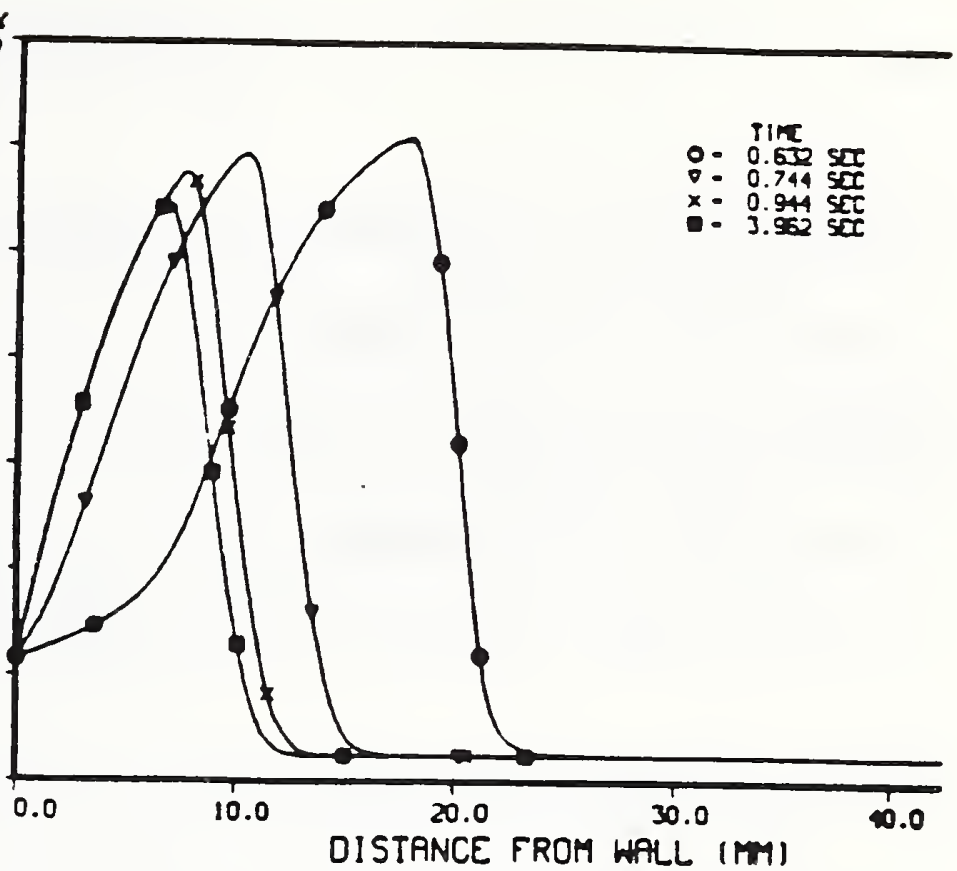

(b)

FIGURE 1.

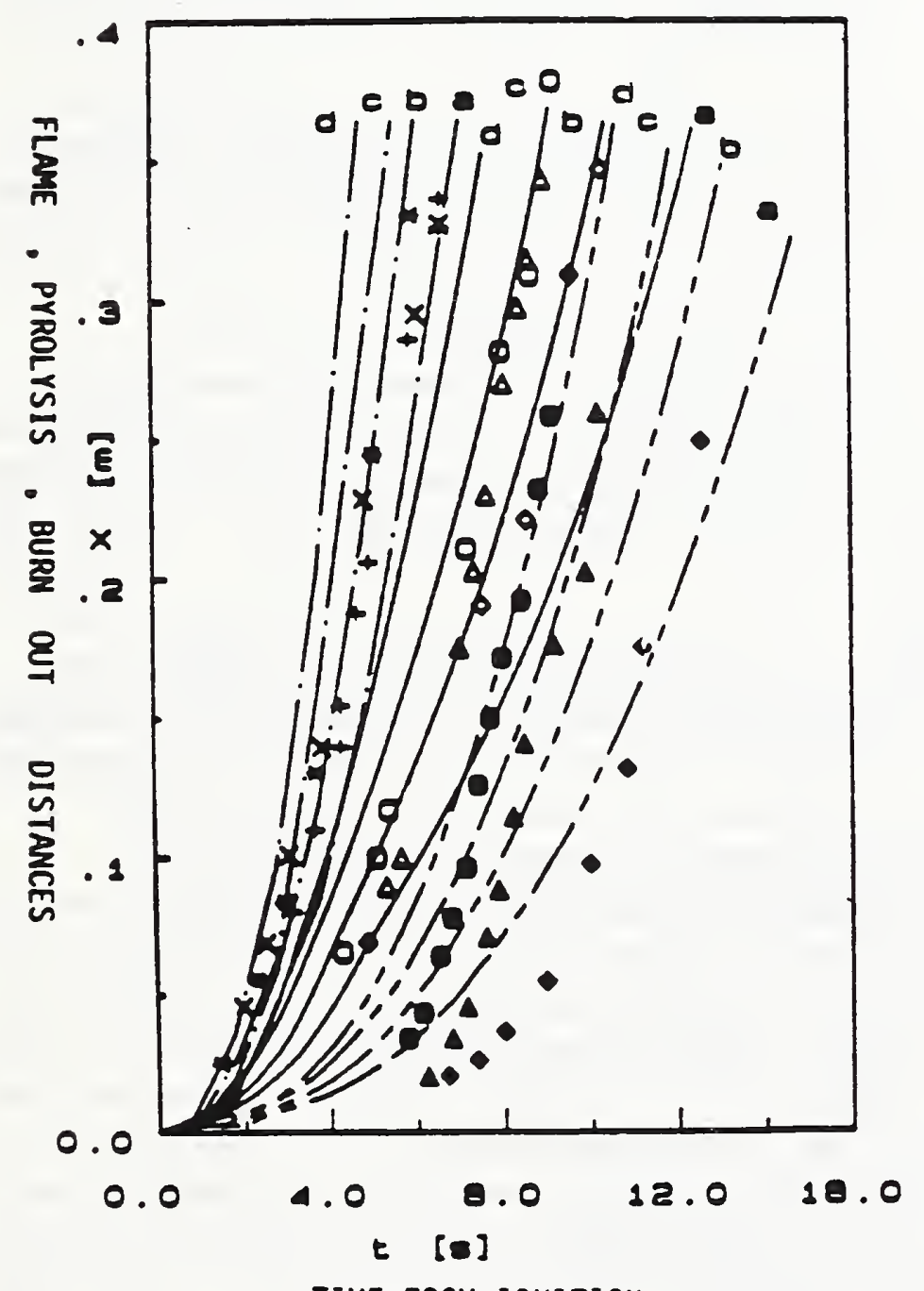

TIME FROM IGNITION

FIGURE 2.

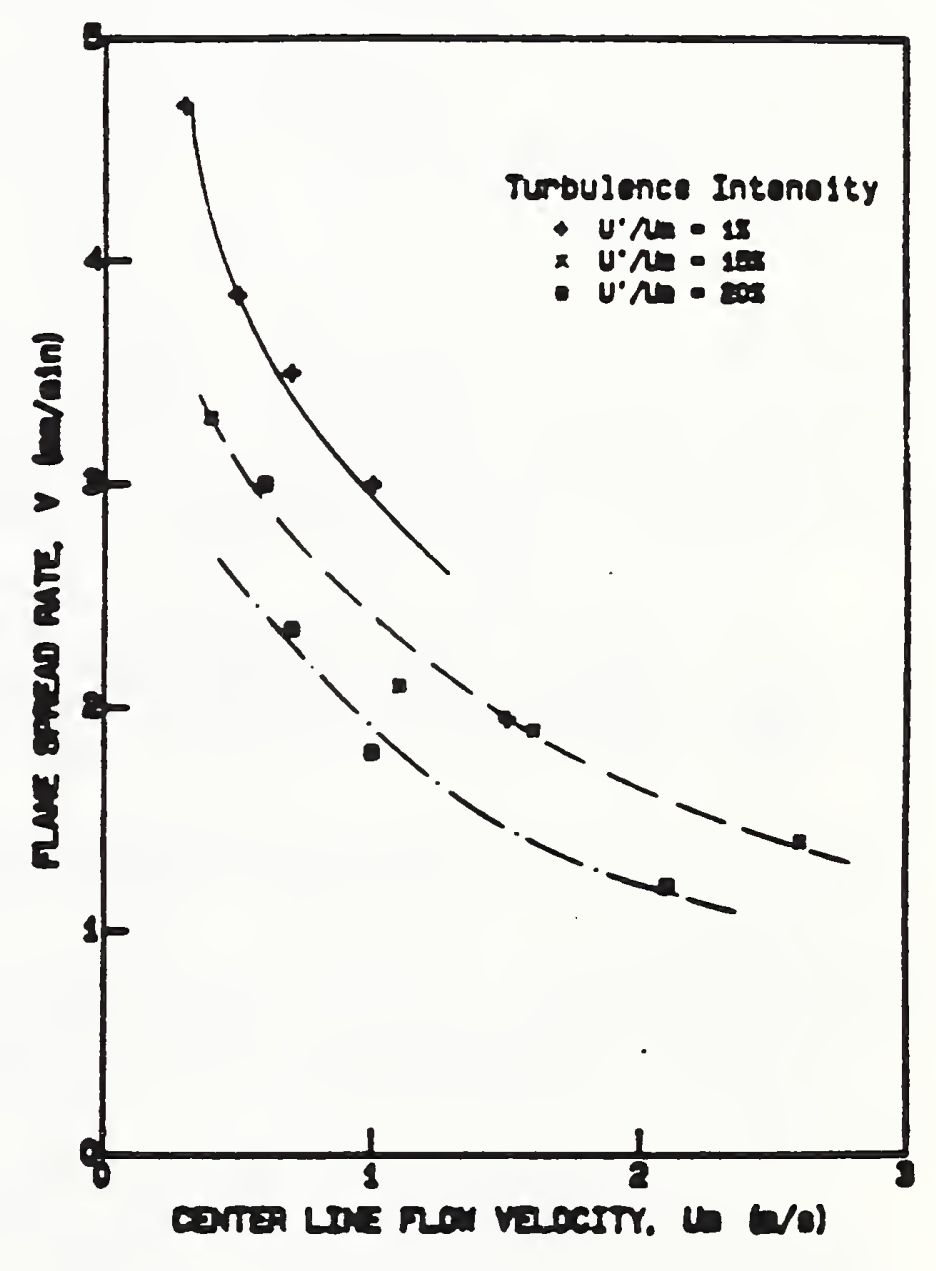

FIGURE 3. 


\section{CENTER FOR FIRE RESEARCH \\ NATIONAL BUREAU OF STANDARDS \\ FY 88}

Institution: The Pennsylvania State University

Grant No.: 60NANB4D0037

Grant Title: Upward Flame Spread on Vertical Wall

Principal Investigator: A. K. Kulkarni

Department of Mechanical Engineering

The Pennsylvania State University

University Park, PA 16802

Other Professional Personnel: S. Fischer, NBS

R. Vettori, NBS

Scientific Officer: $\quad$ K. D. Steckler

Technical Abstract:

Introduction: Upward flame spread is highly significant for fire growth because it is more rapid than lateral or downward spread in wall fires. An experimental and theoretical study of upward flame spread on vertical walls was initiated this year. The overall objective is to develop a predictive methodology to determine if there is sustained upward spread on a given material, and if so, to calculate the upward flame spread velocity using the known or easily measurable properties for that material. Specifically, a model for upward spread is developed and experiments are designed and conducted in order to provide proper input to the model and to validate the model

capabilities. Such an algorithm for the prediction of upward flame spread will allow zone models to evaluate the fire growth and hazard in buildings and compartments more accurately and also it will allow a quantitative assessment of flammability of materials burning in vertical orientation.

Development of Mathematical Model: Concepts introduced in the upward flame spread models of Saito et al.(1985) and Quintiere et al. (1986) have been developed further to formulate a model that accepts certain measurable "fire properties" obtained from small scale experiments in order to predict the upward propagation of fire on a vertical wall made of a combustible material. The present formulation differs from the previous models in primarily two respects, (a) the present model accepts an arbitrarily specified local mass loss rate as a function of time, unlike the previous models which assumed $\dot{m}^{\prime \prime}(t)$ to be either a constant or an exponentially decaying function, and (b) the present model allows as input a flame height correlation in the form of $x_{f}=K Q^{\prime}$ (where $x_{f}$ is flame height, $\dot{Q}^{\prime}$ is energy release rate per unit length, and $K$ and $n$ are constants), unlike previous models which assumed $n=l$ and simplified the analysis considerably. The other quantities required as input to the model are, (a) the ignition temperature and the product of thermal conductivity, density, and heat capacity for the material, which are obtained from the LIFT apparatus (Quintiere et al. 1986), and (b) a numeical value for flame heat feedback. Model equations are solved numerically on a personal computer to obtain the upward flame velocity, $V(t)$, and the 
progression of pyrolysis and burnout fronts ( $x_{p}$ and $x_{b}$ ) as a function of time. Development of the model is in progress.

Mass Loss Rate Measurements: The mass loss rate should be determined under similar conditions as the upward flame spread situation. Specifically, the sample must burn in the same orientation (vertical) and be uniformly covered with flames, similar to the unburnt material above the pyrolysis front. In order to obtain such data, a small scale test apparatus was designed and fabricated as illustrated in Fig. 1 . A $20 \mathrm{~cm} \times 7.5 \mathrm{~cm}$ sample is held flush in an inert, surrounding wall. The sample is subjected to flames from a natural gas line burner positioned parallel to the lower edge of the sample, well below the sample. Flames from the burner uniformly cover the sample surface, simulating wall material which is covered by flames issued by the upstream, burning surface.

One of the objectives of this series of experiments is to systematically study the influence of burnout time, $\tau_{b}$, on the process of upward flame spread. This means samples are thin for a small $\tau_{b}$. However, in order to simulate a typical wall fire, the samples must burn as "thermally thick" solids. (For example, a thin wall panel burns on the exposed surface with a heat loss into the interior of the wall, and therefore it behaves approximately like a thermally thick solid.) Hence, the thin samples (such as a 1/16" PMMA sheet, a cardboard, a textile material, or a wall panel) are first mounted on a thicker substrate, typically a 1/2" marinite panel.

The weight of the sample is continuously recorded as $m(t)$. The data are smoothed using a 31-point averaging scheme and curvefitted to a fifth order polynomial. The mass loss rate per unit area, $\dot{m}^{\prime \prime}(t)$, is then expressed as,

$$
\dot{m}^{\prime \prime}(t)=a_{4} t^{4}+a_{3} t^{3}+a_{2} t^{2}+a_{1} t+a_{0}
$$

Materials tested in this apparatus so far are, PMMA slabs of 1/16", 1/8" and $1 / 2 "$, particle board, polystyrene, and polyvinylchloride. Some of these samples did not yield satisfactory results because of dripping or delaminating problems. Additional samples to be tested are, textile materials of various types, cardboards of various thicknesses, wall panels, and foam materials.

Larger Scale Experiments: In order to verify the model, upward flame spread experiments are being conducted on $45 \mathrm{~cm} \times 240 \mathrm{~cm}$ samples. Here the important tasks are:

(a) to measure $V(t)$ and $V\left(x_{p}\right)$ for various materials

(b) to measure forward heat flux (ahead of the pyrolysis front), q", as a function of time and distance

(c) to conduct a systematic study with the burnout time as a parameter

(d) to classify materials that sustain/do not sustain upward propagation (Go/No go tests) and compare the results with predictions.

Figure 2 shows a schematic of the setup. Ignition is achieved by a linear gas burner. Heat flux to the wall is measured by five total heat flux gages. The test run is video-taped and $x_{f}$ is inferred from the recording. The pyrolysis front is followed visually during the test run, indicated with a pointer, and recorded on the video camera. These experiments are currently in progress. 
Mass loss rate for a $0.5^{\prime \prime}$ thick particle board was measured in the small scale apparatus described above and plotted in Fig. 3 after smoothing and curvefitting. Using available properties and the measured mass loss rate as input to the model described earlier, upward flame spread velocity and pyrolysis height were calculated as a function of time. The maximum pyrolysis height, $x_{p, m a x}$, as a function of the igniter strength is compared with the data of Saito, et al. (1985) in Fig. 4. Comparisons are made for two different values of $K$ (the constant appearing in the flame height correlation). The value of $\mathrm{K}$ for 50 f flame tip intermittency height has been reported to be 0.0433 , and for continuous flame it is reported to be 0.027 , with the same exponent of $n=2 / 3$ (all units in meters) (Quintiere et al. 1986). Using these two values of $K$, predictions are found to overestimate and underestimate the data, respectively. At 50 intermittency, the prediction of $x_{f}$ is high because the heat flux to the wall starts dropping off before $x=x_{f}$; for continuous flame, $\dot{q}^{n}$ is significant at least up to $x=x_{1}$. The present model assumes a constant $\dot{q}$ " up to $x=x_{f}$ and then zero thereafter. Therefore, the prediction of $x_{p}, x^{2}$ is consistent with respect to the $k$ values and the model appears to predict data well.

\section{References :}

Quintiere, J. and Harkelroad, M. (1984) New Concepts for Measuring Flame Spread Properties. NBS report no. NBSIR84-2943.

Quintiere, J., Harkelroad, M., and Hasemi, Y. (1986) Comb. Sci. Tech. 48, 191.

Saito, K., Williams, F. A., and Quintiere, J. (1986) Upward Turbulent Flame Spread. Fire Safety Science, Proc. First Int'l. Symp., Hemisphere Pub., 75.

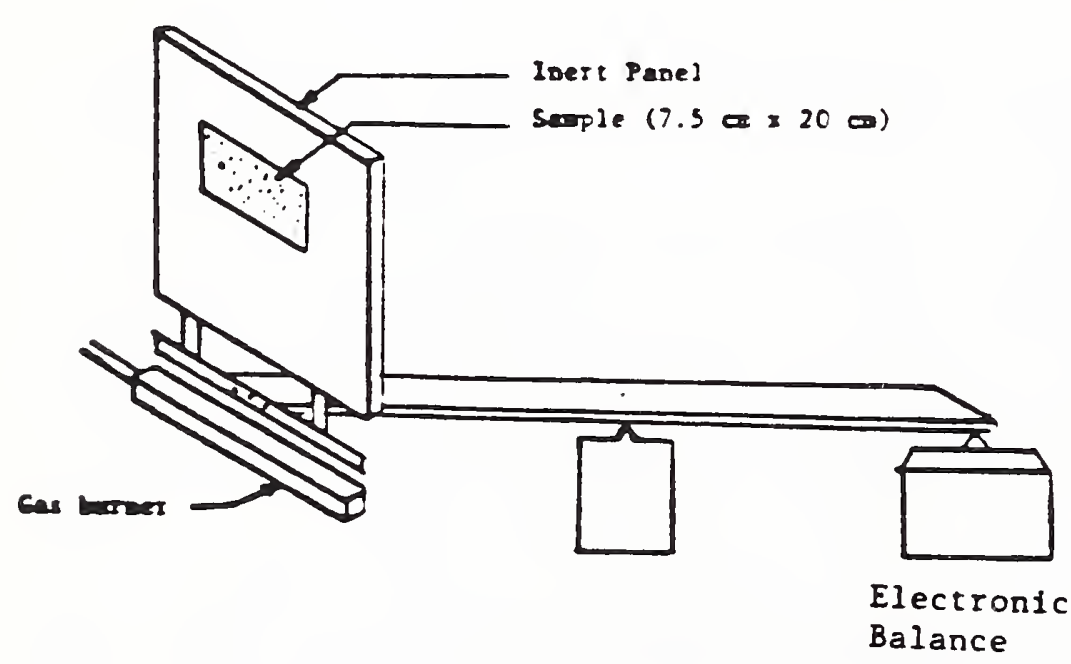

Fig. 1 : A schematic of mass loss rate experimental setup

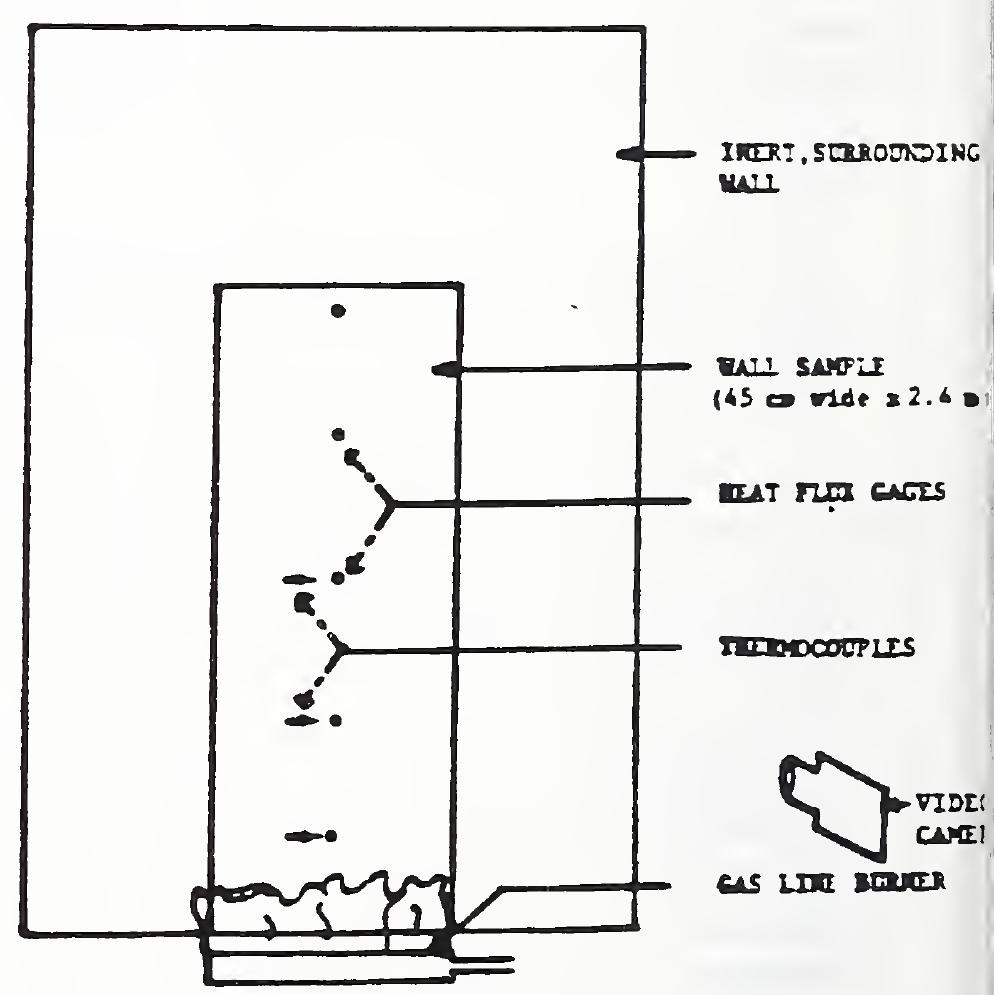

Fig. 2 : Apparatus for upward spread experiments 


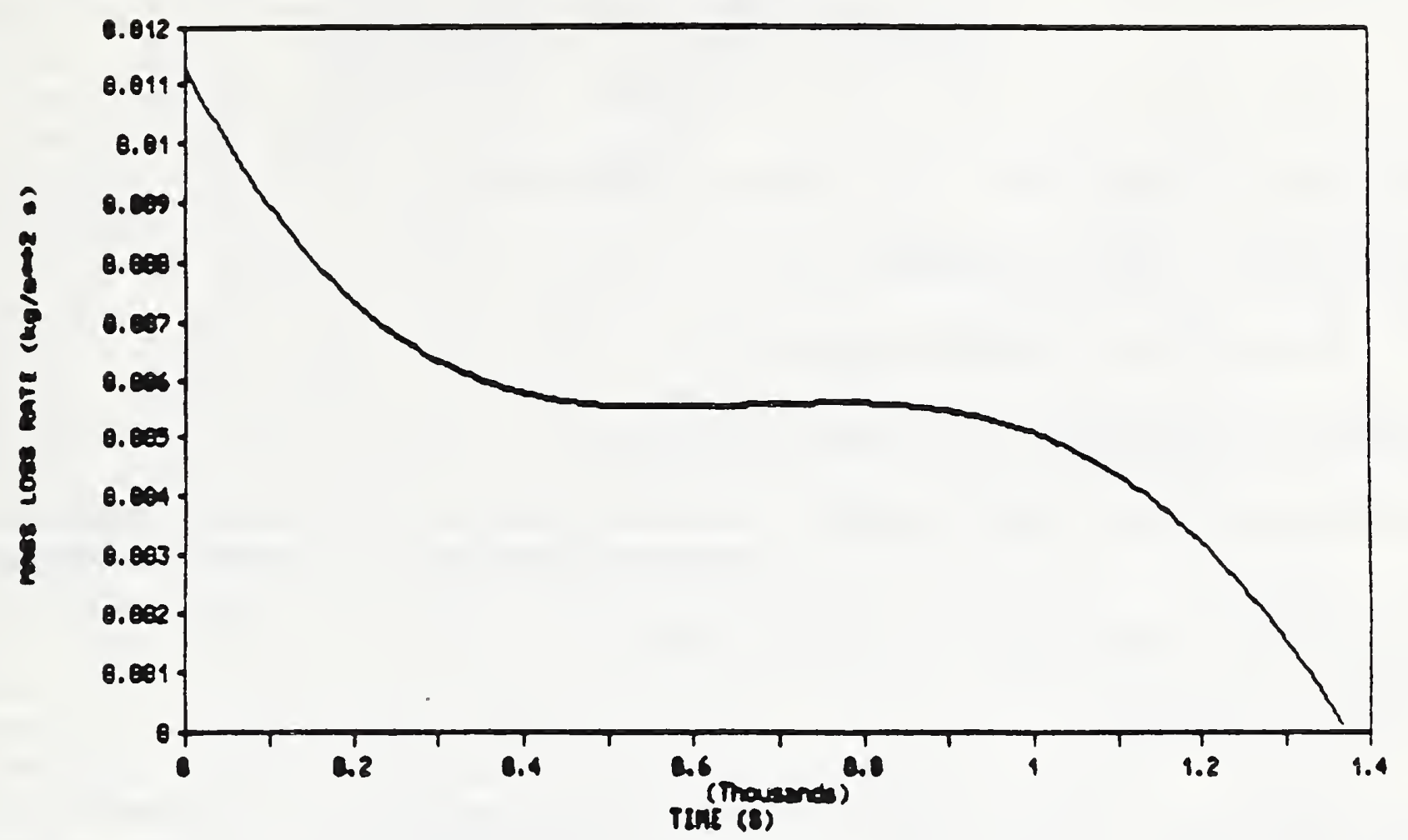

Fig. 3 : Mass loss rate as a function of time for particle board. (Polynomial curve fit)

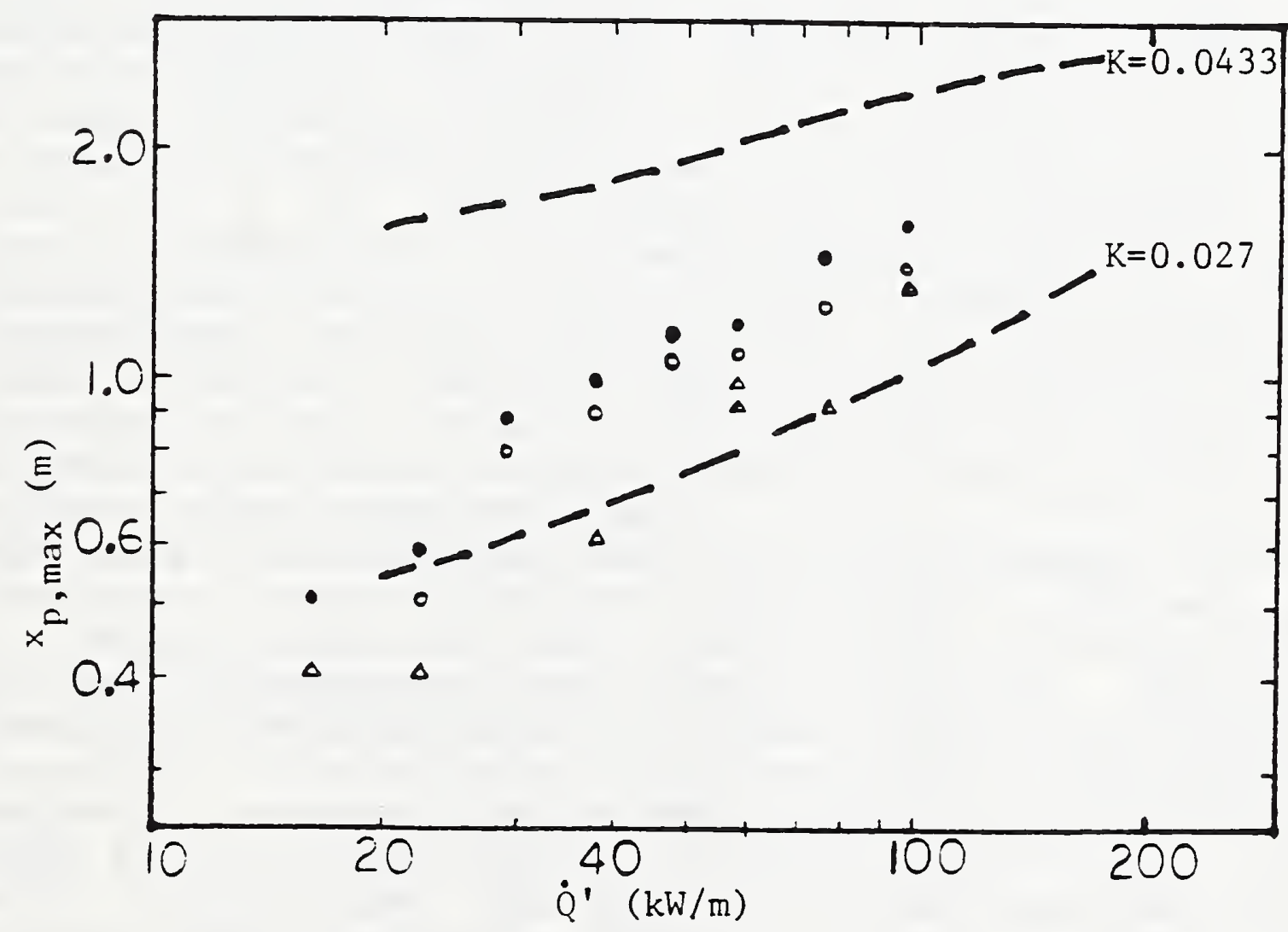

Fig. 4 : Pyrolysis front height as a function of burner energy supply rate for particle board. Symbols are data of Saito et al. (1985) for three different definitions of pyrolysis front; dashed lines are predictions using present model. 
CENTER FOR FIRE RESEARCH

NATIONAL BUREAU OF STANDARDS

FY 88

Institution: TRW Space and Technology Group

Contract NO.: 6ONAN37D0742

Title: Wind-Aided Flame Spread

Principal Investigator: Francis Fendell

other Professional Personnel: Gerald Butler, Co-Participant George Carrier, Co-Participant

NBS Scientific Officer: Howard Baum

Technical Abstract:

Objectives A definitive (numerical) solution is sought to a prototypical formulation of wind-aided flame spread over a solid fuel slab. In particular, the rate of increased involvement is sought as a function of the (given) ambient thermohydrodynamic environment (temperature, wind speed, air vitiation, etc.) and of the (given) physicochemical properties of the fuel slab (density, heat capacity, thermal conductivity, temperature for onset of pyrolysis, endothermicity required for pyrolysis, exothermicity released by combustion of the gaseous pyrolyzate, etc.). The formulation is a straightforward generalization, for ignition at the "leading edge" of a thermally thick slab, of the classical treatment by Emmons (1956) of the steady-state diffusion-flame burning of a fully involved slab; accordingly, the solution to the initial/boundary-value problem treated here evolves in time to the steady-state Emmons solution by contruction.

The motivation for pursuing careful finite-difference solution to this three-independent-variable problem [time since ignition, distance downwind from the leading edge, distance transverse to the two-phase (gas-solid) interface) are several. First, it is of interest to ascertain precisely what such a "simplest reasonable" formulation of the physics predicts about the rate of fire spread under forced-convective aiding of the fire propagation. This is one of the most escape-limiting scenarios associated with fires in structures. Second, experimental results obtained by Loh and Fernandez-Pello (1984) are available for the problem under theoretical study. Ioh and Fernandez-Pello suggested that the above-described formulation adequately incorporates the physics, so that the formulation yields results consistent with the measurements reported for noncharring (PMMA-type) polymers. The present investigators are concerned whether indeed the status of this particular cornerstone of fire science and technology is in such a satisfactory state: perhaps a careful solution of the formulation will prove to be at odds with their rough approximate solution, which agrees with the experiment. If so, then is the execution of the experiment or the adequacy of the formulation deficient? 
other motivations are as follows. First, this problem is highly reminiscent of the ASTM E84 protocol, executed in a steiner tunnel. Abuse and misuse of data from this protocol is irrelevant to the present discussion. What is relevant is that the pursuit of this modeling supports by example the contention that a useful companion model can be developed for even seemingly intricate protocols. Such models establish what physicochemical properties of the test sample are central to the material performance in the protocol. That is, the model helps to elucidate what the E84 protocol does, and does not, reveal about the fire behavior of a sample. Ultimately, in the distant future, such models may become sufficiently predictive to obviate the need for some testing. second, it is logical to have the relatively simplistic case of a noncharring material well in hand before pursuing the additional complications of (I) a charring and soot-forming material, with the attendant complications of significant radiative transfer of heat, or (2) the more closely coupled dynamics and energetics of fire spread up a vertical wall, another wind-aided-fire-propagation scenario. Third, fire spread under forced-convective-flow assistance arises in many ominous structure fires, often in connection with ceiling-type spread along a long corridor-like expanse, owing to effluents from flashover in an adjoining room (e.g. the MGM Grand casino fire).. Thus, a capacity for parameterization of this "subscaled" phenonomenon into multicompartment-structure-fire hydrocodes is highly desirable.

Approach A so-called stefan problem arises from the formulation of the gas-phase and solid-phase energetics, coupled by constraints holding at the two-phase interface. A stefan problem entails a moving boundary; identifying the position of this boundary as a function of time is a key output of the solution. Here the moving boundary is a "separatrix" at the two-phase, gas-solid interface. Downwind of the separatrix is the preheating zone: the wash of hot gases raises still-uninvolved portions of the solid slab toward the temperature at which occurs the outgasing of combustible fuel vapor from the solid. Upwind of the separatrix is the pyrolysis zone: the endothermic requirement for generation of fresh pyrolyzate is met by the exothermicity from the gas-phase diffusion flame. Thus, the separatrix is the furthest-downwind position along the two-phase interface at which the temperature is the pyrolysis temperature. Alternatively, the separatrix is the furthest-upwind position along the two-phase interface at which the interphase mass transfer is zero.

In the present formulation, the problem admits a similarity solution, such that the three independent variables may be reduced to two independent variables. Explicitly, the solution depends on two ratios of the three independent variables: the ratio of streamwise coordinate to time, and the ratio of transverse distance to the square root of streamwise distance. This similarity property is not exploited, but rather is reserved as a check on the accuracy of numerical results obtained with a three-independentvariable formulation. The reason is that numerical techniques are being developed robust enough to treat in the future more difficult situations in which the simplications of a similarity property no longer are available. However, the similarity property assures that the ratio of the propagation speed of the separatrix 
(hereafter identified as the fire-spread rate) to the ambient wind speed is (conveniently) constant in space and time for the particular cases examined in this discussion. The normalized (nondimensionalized) -rate of fire spread depends on ten dimensionless parametric ratios. Ultimately one would like to identify the dependence of the normalized spread rate on each of these ten parametrical ratios, but, in the interim, attention is concentrated on those parameters varied in the available experimental data. In fact, only one dimensionless ratio is varied in the available data, the ambient oxygen mass fraction (in other words, the degree of vitiation of the aiding wind). The magnitude of the aiding wind is also varied in the experiments, but this dimensional parameter enters into the nondimensionalization of the spread rate only, and into none of the dimensionless ratios (in the present formulation, which adopts inviscid flow). Thus, the experimentally observed linear increase of spread rate with increase in the ambient wind speed follows directly from dimensional analysis (without explicit solution) of the current formulation.

Results Thus, a large fraction of the twenty executed computational cases are dedicated to identifying how the fire-spread rate varies with the ambient oxygen mass fraction, with the nine other dimensionless ratios of parameters held invariant. According to the experimental observations of Loh and Fernandez-Pello and also according to their approximate solution of the inital/boundary-value problem posed here, the dependence of the spread rate on the ambient oxygen mass fraction is very nearly quadratic (over the range for which a finite rate of fire spread is observed). Numerical results previously reported (Butler, Carrier, and Fendell 1985) for the present model yielded a linear increase of spread rate with ambient oxygen mass fraction, but these results pertained to a sample of far less density than the density of the PMMA examined in the experiment.

\section{References}

Butler, G., Carrier, G., and Fendell, F. 1985 Modeling of wind-aided flame spread. Report 39641-6001-UT-00. Redondo Beach, CA: TRW Space and Technology Group.

Carrier, G. F., Fendell, F. E., and Feldman 1980 wind-aided flame spread along a horizontal fuel slab. Comb. Sci. Tech. 23, 41-78.

Emmons, H. W. 1956 The film combustion of liquid fuel. Z. Angew. Math. Mech. 36, 60-71.

Loh, H.-T., and Fernandez-Pello, A.C 1984 A study of the controlling mechanisms of flow assisted flame spread. (International) on Combustion, 1575-1582. Twentieth symposium Combustion Institute. Fittsburgh, PA: 


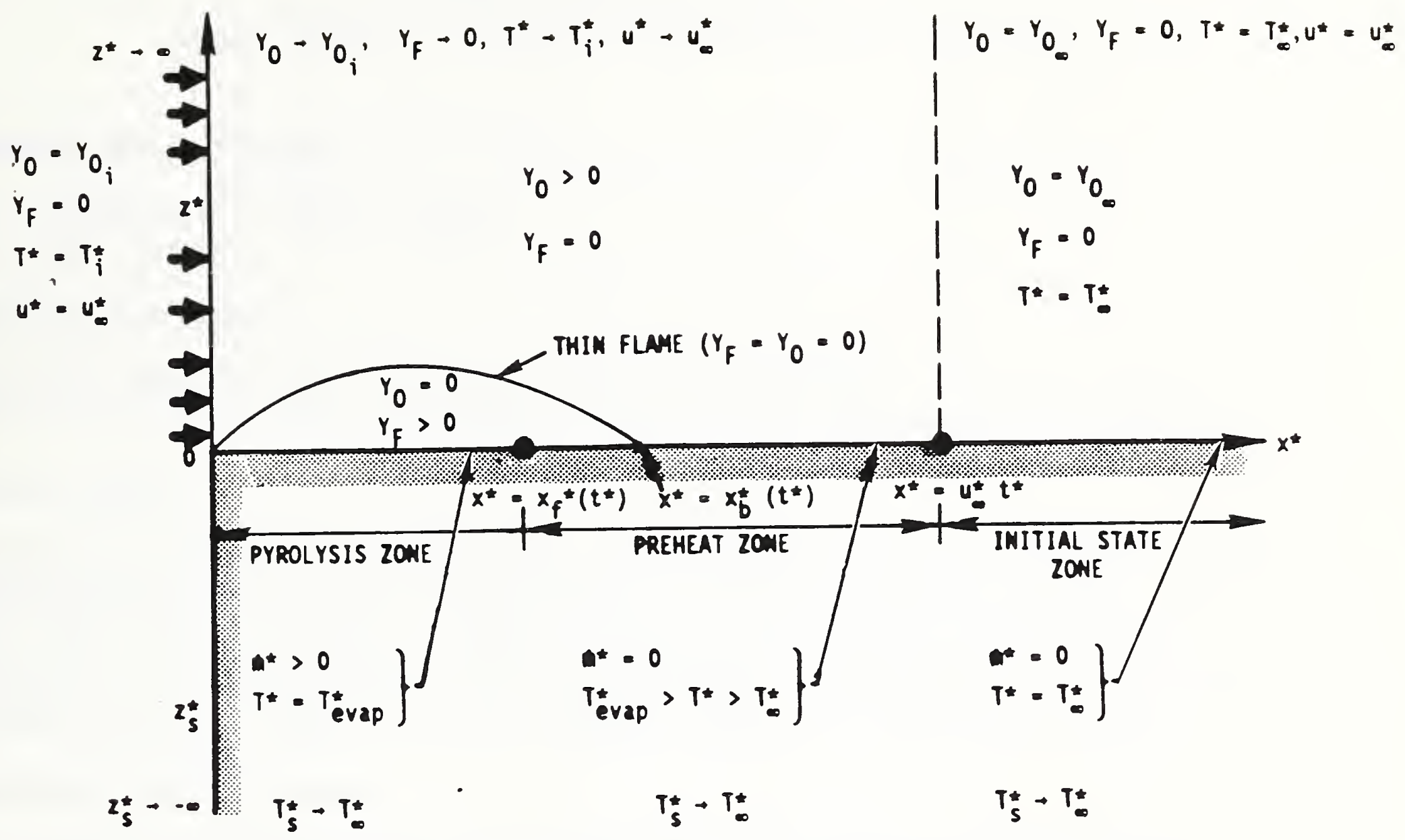

Figure 1. A schematic of a fundamental model of wind-aided tire spread across a solid slab, semi-infinite in length and thickness. A super asterisk denotes a dimensional quantity; absence of the asterisk, a dimensionless quantity. The streamwise coordinate is $x^{*}$; the (densityweighted) transverse coordinate in the gas phase is $z *(>0)$ and $z *(<0)$ in the solid phase; the time since onset of heated flow is $t^{*}$. In the interfacial plane $z *=z^{*}=0$, the preheat zone extends from the hot-gas front $x *=u * t * t 0^{s}$ the pyrolysis front $x *=x *(t *)$. The pyrolysis zone extends from the pyrolysis front to the leading edge of the solid slab $x^{*}=0$. The pyrolyzed-vapor-sustained thin gas-phase diffusion flame attaches to the interface at $x *=0$ and at $x^{*}=x_{b}^{*}\left(t^{*}\right)$, where in general $u_{\infty}^{*} t *>x_{b}^{*}(t *)>x_{f}^{*}(t *)>0$. The flame locus is defined by $Y_{F}=Y_{O}=0$, where $Y_{F}$ and $Y_{O}$ are the stoichiometrically adjusted mass fraction for fuel vapor and oxygen, respectively. The temperature in the gas phase is denoted $T *$, and in the solid phase, $\mathrm{T}_{\mathrm{s}}^{*}$. The ambient gas and solid are both at $\mathrm{T}_{\infty}^{*}$. The fuel-vapor-free heated vitiated-air flow is characterized by values $\mathrm{Y}_{\mathrm{O}_{1}} \mathrm{T*}$, and $u *{ }_{\infty}$ the speed of the inviscid flow. The local values of the interfacial mass transfer $\dot{m} *$, in units of mass per area per time, and interfacial temperature are noted, where $\mathrm{T}_{\text {evap }}^{*}$ is the pyrolysis temperature. The rate of flame spread is associated with $\mathrm{dx} \underset{\mathrm{f}}{*} / \mathrm{dt*}$. 



\section{Professional Personnel}

T. J: Ohlemiller, Project Leader

S. Dolan, Chemist

\section{Project Objectives}

To further our fundamental understanding of smoldering combustion phenomena while, at the same time, providing input data for hazard analysis models.

\section{Project Scope}

Smoldering combustion is responsible for a large fraction of residential fire deaths and it appears that $C O$ poisoning is a major underlying cause. We have thus undertaken a program to characterize the emissions from this mode of combustion for some common residential fuels. At the same time, we are exploring the smoldering behavior of these fuels between the extinction and flaming limits in order to obtain insights into the controlling factors.

\section{Technical Accomplishments}

The initial work has focused on the smoldering of solid wood since this dovetails with prior work for DOE. A first set of experiments has been completed in which samples of red oak and white pine were made to undergo self-sustained smolder under conditions of controlled air supply. Achievement of self-sustained smolder over solid wood requires a configuration which limits the heat loss rate from the oxidizing char surface; propagation along the interior surface of a U-shaped channel was used here. The first results are all for the case in which the smolder zone propagates in the same direction as the air flow along the channel. The range of air flow rates for which stable propagation is possible in the current configuration is rather narrow - - about 8 to $18 \mathrm{~cm} / \mathrm{sec}$ referred to the initial channel cross section. Transition to flaming occurs with higher flow rates and extinction occurs with lower flow rates. In the range of stable smolder, CO comprises 3 to 4 mole 8 of the exhaust gases evolved from the combustion chamber and 10 to 20 weight $\%$ of the evolved mass from the wood. Other products have also been characterized and compared with those evolved from the same woods in other modes of gasification. In addition we have obtained estimates of the rate of heat release from this smolder situation on the basis of oxygen consumption. Analysis of temperature profiles in the wood during smolder propagation leads to the inference that radiation along the channel axis is a major heat transfer mode driving the propagation. Future work includes expanding the range of configurations for solid wood and examination of $C O$ from the smoldering of other residential fuels, particularly upholstery materials. Plans also include the development of a model for smolder propagation in twodimensional slab configurations.

Reports and Publications 
Ohlemiller, T. and Shaub, W., "Products of Wood Smolder and Their Relation to Wood-Burning Stoves", National Bureau of Standards NBSIR 88-3767, May, 1988

\section{Related Grant}

"Kinetics of Oxygen Chemisorption and Desorption of Combustion Products in the Smoldering Combustion of Cellulosic Material", G. Richards, U. of Montana 


\section{CENTER FOR FIRE RESEARCH \\ NATIONAL BUREAU OF STANDARDS}

FY 88

Institution: University of Montana

Grant. Number: 60NANB5D0554

Grant Title: Kinetics of Oxygen Chemisorption and Desorption of Combustion Products in the Smoldering Combustion of Cellulosic Material

Principal Investigator: Professor Geoffrey N. Richards

Wood Chemistry Laboratory

University of Montana

Missoula, Montana 59812

(406) 243-4435

Other Professional Personnel: Dr. F.-Y. Hshieh, Postdoctoral Research Associate

Mr. William F. DeGroot, Research Specialist

NBS Scientific Officer: Dr. Thomas Ohlèmiller

Technical Abstract:

Introduction. Smoldering combustion is a major source of ignition of highly destructive residential and forest fires. Depending on the prevailing conditions, smoldering is propagated by oxidation of a stable char formed either by nonoxidative thermal degradation (pyrolysis) or by oxidation occurring simultaneously with the initial thermal degradation reactions. In either case, the process must involve combination of oxygen with the substrate and evolution of both pyrolysis and combustion products. The tendency of a material to support smoldering combustion has previously been correlated with the kinetics of oxygen chemisorption at low temperatures on stable chars formed from cellulose under pyrolytic conditions.

The main purpose of this research was to study the kinetics of pyrolysis and of oxygen chemisorption and desorption of combustion products under conditions which occur in the smoldering combustion of lignocellulosic materials, which were expected to behave differently from pure cellulose. Previous work (FY 87) was concerned with (1) first chemical events in pyrolysis and oxidative pyrolysis of wood at $250^{\circ} \mathrm{C}$, and (2) influence of indigenous and of added metal ions in pyrolysis and oxidative pyrolysis of wood from room temperature to $550^{\circ} \mathrm{C}$ at low heating rate $\left(3^{\circ} \mathrm{C} / \mathrm{min}\right)$. During the present period of study, work was carried out in the following areas: (1) factors influencing chemisorption of oxygen and ignition of wood chars, (2) factors influencing chemisorption of oxygen and ignition of chars from newsprint (newsprint was studied because it is frequently the raw material for manufacture of cellulosic insulation).

Factors Influencing Oxygen Chemisorption and Ignition of Wood Chars. Solid discs of cottonwood have been pyrolyzed under nitrogen to an appropriate temperature (HTT), and then cooled under nitrogen to 
chemisorption temperature for study of oxygen chemisorption activity (CSA). Presorption of nitrogen slowed the initial rate of oxygen chemisorption, but had no effect on long-term ( 4 hours) chemisorption. Variation of HTT lead to a pronounced maximum CSA at $\operatorname{HTT} 450-500^{\circ} \mathrm{C}$; the causes of this effect are assumed to be chemical in origin and have been studied further by FTIR of the chars. Chars have also been prepared for collaborative solid-state NMR at the University of Utah and publication of the results is anticipated in the future. Removal of indigenous metal ions from the wood and replacement with individual metal ions resulted in small but significant changes in CSA.

Ignition of the char samples prepared by pyrolysis in nitrogen has been studied without removal of the char from the thermal balance. The ignition temperatures of chars were dramatically influenced by the nature of the cations present. The complete removal of cations was effective in raising ignition temperature. The physical form of the wood samples (i.e. fine and coarse powders and solid discs) also had considerable influence on ignition temperatures, with the chars from solid wood samples igniting at lower temperatures than corresponding chars from wood powder, either in oxygen or in air.

Factors Influencing Oxygen Chemisorption and Ignition of Chars from Newsprint. The oxygen chemisorption behavior of newsprint chars is similar to that of wood chars and significantly different from cellulose chars. Maximum chemisorption activity occurred with chars prepared at $500^{\circ} \mathrm{C}$. The black ink on newsprint has a retarding effect on the ignition of chars, but color in newsprint enhances the ignition of chars due to the concomitant increase in metal ion content. The lowest "minimum ignition" temperature (i.e. without prior low temperature oxygen chemisorption) occurred with chars of $\mathrm{HTT} 375^{\circ} \mathrm{C}$. For higher temperature chars (HTT $\geq 375^{\circ} \mathrm{C}$ ), the ignition temperature increased as heat treatment temperature increased. These effects are interpreted in terms of the more ready oxidation of aliphatic than aromatic components of chars and of the influence of HTT on chemisorption activity.

This project was completed on April 30, 1988. A new project, "Chemical Factors Influencing Ignition and Combustion in Lignocellulosics" began May 1, 1988. Research funded by this project will involve (1) contribution of cellulose, hemicelluloses and lignin to combustion and ignition properties of lignocellulosic chars, (2) properties of low temperature lignocellulosic char ("protochar"), and (3) interaction and effects of oxygen chemisorption and metal ions on ignition and combustion of lignocellulosics.

\section{Reports and Papers}

1. W.F. DeGroot and G.N. Richards, "The Influence of Pyrolysis Conditions and Ion-Exchanged Catalysts on the Gasifization of Cottonwood Chars by Carbon Dioxide," Fuel, 67 (1988) 352-360.

2. W.F. DeGroot and G.N. Richards, "The Effects of Ion-exchanged Cobalt Catalysts on the Gasification of Wood Chars in Carbon Dioxide," Fuel, 67 (1988) $345-351$.

3. T.L. Lowary and G.N. Richards, "Vacuum Pyrolysis of Bark from Pinus 
Ponderosa," J. Wood Chem. \& Tech., in press.

4. G.N. Richards, "Effects of Impurities on Degradation of Sucrose under Processing Conditions," book chapter in "Chemistry and Processing of Sugar Cane," Ed. Margaret Clarke (10/19/87).

5. T.L. Lowary and G.N. Richards, "Effects of Impurities on Hydrolysis of Sucrose in Concentrated Aqueous Solution," Int. Sugar Journal, in press.

6. M. Essig, T. Lowary, G.N. Richards and E. Schenck, "Influences of "Neutral" Salts on Thermochemical Conversion of Cellulose and of Sucrose," Proceedings from the Biomass Conversion Conference, Phoenix, 1988 .

7. F.-Y. Hshieh and G.N. Richards, "Factors Influencing Chemisorption and Ignition of Wood Chars," submitted to Combustion and Flame, 2/88.

8. W.F. DeGroot, T.H. Osterheld and G.N. Richards, "The Influence of Natural and Added Catalysts on the Gasification of Wood Chars," Proceedings from the Biomass Conversion Conference, Phoenix, 1988.

9. W.F. DeGroot, M.P. Kannan and G.N. Richards and 0 . Theander, "Gasification of Agricultural Residues (Biomass); Influence of Inorganic Constituents," submitted to J.Agric. Food Chem. 3/88.

10. F.-Y. Hshieh and G.N. Richards, "Factors Influencing Oxygen Chemisorption and Ignition of Chars from Newsprint," submitted to Comb. and Flame 3/88.

11. M.D. Rahman and G.N. Richards, "Interactions of Starch and Other Polysaccharides with Condensed Tannins in Hot Water Extracts of Ponderosa Pine Bark," J. Wood Chem. and Tech., 8(1) (1988) 111-120.

12. J.D. Blake, M.L. Clarke and G.N. Richards, "Determination of Organic Acids in Sugar Cane Process Juice by High-performance Liquid Chromatography: Improved Resolution using Dual Aminex HPX-87H Cationexchange Columns Equilibrated to Different Temperatures," J. Chromat., 398 (1987) 265-277.

13. F.-Y. Hshieh and G.N. Richards, "Factors Influencing Chemisorption of Oxygen and Ignition of Wood Chars," Prepr. Pap., Combined Meetings, Eastern Section: The Combustion Institute 20 th Fall Technical Meeting, Chemical and Physical Processes in Combustion and the National Bureau of Standards Annual Conference on Fire Research, Washington, D.C., November 1987.

14. G.N. Richards, "Kinetics of Oxygen Chemisorption and Desorption of Combustion Products in the Smoldering Combustion of Cellulosic Materials," Final Report, NBS Grant No. 60NANB5D0554, Wood Chemistry Laboratory, University of Montana, Missoula, Montana, April 1988. 
PART I. Basic Research and Tools for "Engineered" Fire Safety (In-House Projects and Associated Grants Funded by , NIST)

B. FLAME DYNAMIC PROCESSES 



\section{TURBULENT COMBUSTION SIMULATION}

Professiorial Personnel

Howard R. Baum, NIST Fellow, Project Leader

Daniel M. Corley, Physicist

Ronald G. Rehm, NIST Fellow

Objective

Develop a fundamental understanding of the mechanisms which control gas phase combustion processes in fires and a predictive capability which will allow the evolution of these processes to be calculated from basic principles.

\section{$\underline{\text { Scope }}$}

A theoretical and computational approach to the study of the transport, mixing, diffusion, and reaction processes in an enclosure fire context. Each process is considered in detail at the appropriate level of description. Since these phenomena occur at widely different length and time scales, a large part of this effort is devoted to providing a set of overlapping analyses in frames of reference (see figures on next page) which permit the phenomena to be coupled together.

\section{Support}

Part of this work, (see items 2. and 3. below) is supported by the Air Force Office of Scientific Research.

\section{Technical Accomplishments}

\section{Large Scale Eddy Simulations}

A three dimensional time dependent inviscid large eddy simulation of the buoyant convection induced by an enclosure fire developed earlier has been revived and ported to a Convex C120 mini supercomputer acquired jointly with the Center for Applied Mathematics to support this project. The simulation, a sample of whose output is shown in the upper figure, resolves the largest (geometric) scales of the turbulent flow. It also calculates the hydrodynamic transport of all subscale processes using the particles shown in the figure. Use of the new computational facility permits flow resolution over a factor exceeding fifty in length and time scales.

\section{Combustion in a Turbulent Eddy}

The small scale mixing and diffusion processes which provide the actual local environment for the chemical heat release is calculated in the local frame of reference. The local mixture fraction variable, representing the fraction of gaseous material present that was once fuel, is determined as a function of the local large scale flow quantities. The distortion of an initially 


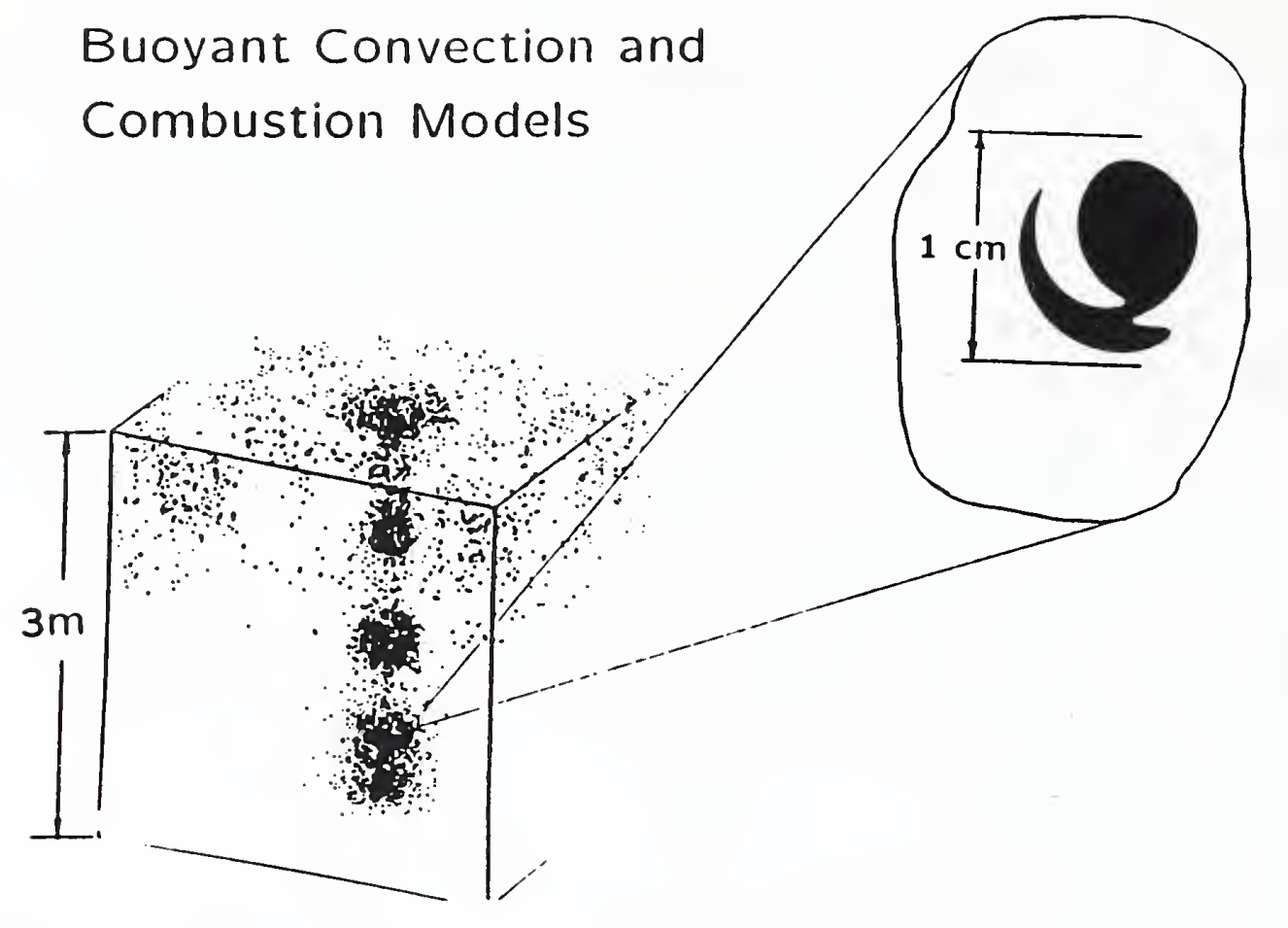

Turbulent Eddy and Chemistry Models

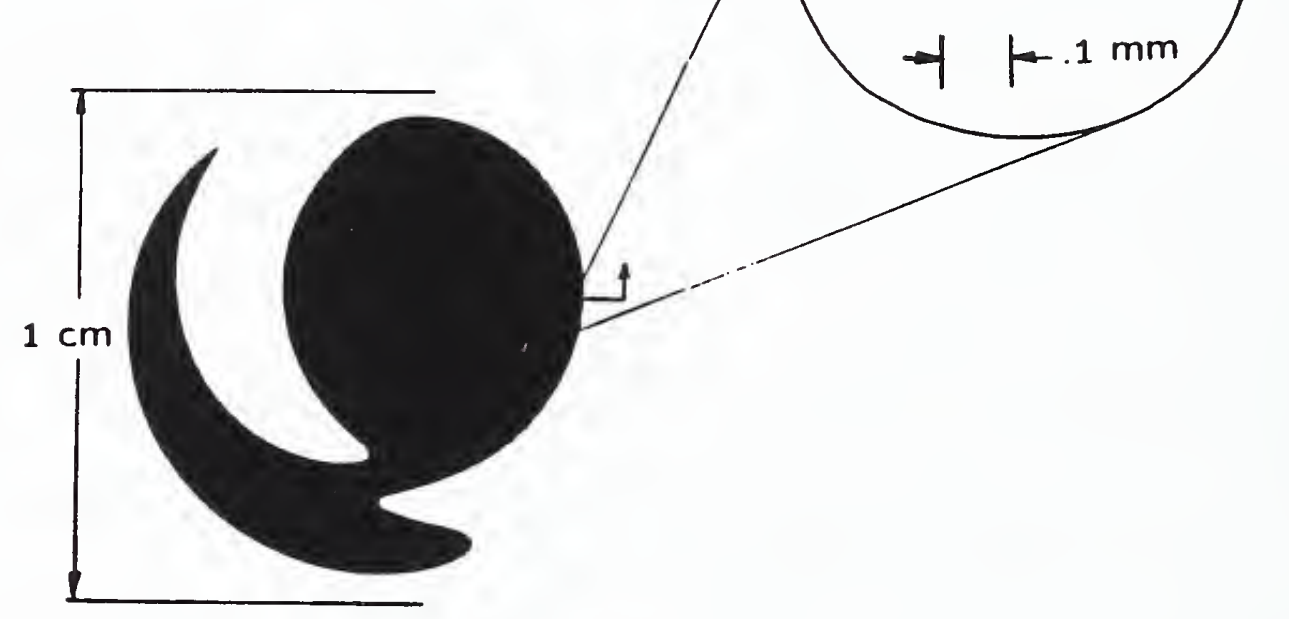


spherical fuel blob under the influence of local mixing is shown in the upper and lower figures. It provides a transition from the room scales down to those where the chemistry occurs. The model on which the solution is based contains the effect of intense local vorticity, feedback from the local heat release, and large density and temperature variations. It is itself a three dimensional time dependent calculation which has also been ported over to the new machine.

\section{Finite Rate Chemistry}

Work has been initiated in collaboration with $\mathrm{Dr}$. John de Ris of Factory Mutual Research Corp. (FMRC) to develop a finite rate hydrocarbon chemistry model for use in conjunction with this program. There are two tasks to be carried out. The first, being performed by Dr. deRis, is to develop an appropriate time dependent solution scheme using the mixture fraction variable as the space-like coordinate. The second, being carried out at NIST, is to imbed a given finite rate chemistry model into the turbulent eddy as indicated in the lower figure.

\section{Reports and Publications}

"Time Dependent Simulation of Small-Scale Turbulent Mixing and Reaction" by Baum, H.R., Corley, D.M., and Rehm, R.G', Twenty First International Symposium on Combustion, The Combustion Institute, Pittsburgh, PA, 1263, 1988.

"Transient Combustion in a Turbulent Fire" by Baum, H.R. and Rehm, R.G., Ninth Joint Meeting of the U.S. - Japan Natural Resources (UJNR) Panel on Fire

Research and Safety, N.H. Jason and B.A. Houston, Eds., National Bureau of Standards Report NBSIR 88-3753, April 1988.

"Diffusion-Controlled Reaction in a Vortex Field" by Rehm, R.G., Baum, H.R., and Lozier, D.W., Symposium in Honor of C.C. Lin. Proceedings, D.J. Benney, F.H. Shu, and C. Yuan, Eds World Scientific, 24l, 1988.

\section{Related Grants}

1. "Subgrid Modeling of the Structure of Transient Diffusion Flames', J. de Ris, FMRC. *

2. "Radiation from Turbulent Lumincies Fires", G.M. Faeth, U. of Michigan. (See p. 130 for description)

*New grant with work just beginning. It is not discussed in this report but will be described in the 1989 publication. 



\section{CENTER FOR FIRE RESEARCH \\ PRIORITY PROJECT - 1988 \\ SOOT FORMATION AND EVOLUTION}

\section{Professional Personnel}

George W. Mulholland, Project Leader

Kermit C. Smyth, Project Leader

Nelson P. Bryner, Chemical Engineer

Paul J.H. Tjossem, Physicist

James Gentry, Guest Scientist

J. Houston Miller, Associate Professor

Thomas Cleary, Guest Scientist

\section{Project Objectives}

Develop scientifically sound principles, metrology, data, and predictive methods for the formation/evolution of smoke components in flames for use in understanding and modeling general fire phenomena.

\section{$\underline{\text { Scope }}$}

This work embraces broad areas underpinning CFR programs with focussed study in the areas of hot gas physics and chemistry. Effects are directed toward improved understanding of the chemical and physical processes which underlie macroscopic fire phenomena and include development of new techniques and methods for studying these processes.

\section{Technical Accomplishments}

During hydrocarbon combustion the exothermic chemical reactions which lead to the formation of water and carbon dioxide consume most of the fuel. However, in many cases a significant fraction of the hydrocarbon fuel is converted into species which participate in chemical growth reactions. These processes lead to dramatic consequences in numerous combustion environments. For example, the formation of intermediate hydrocarbons, such as polycyclic aromatic hydrocarbons (PAH), occurs under fuel-rich conditions and poses a potential long-term health hazard since many $\mathrm{PAH}$ are carcinogenic. In addition, such compounds are also involved in further growth reactions to form soot particles. Radiation from soot dominates energy transfer from large fires, and thus soot formation plays a key role for combustion efficiency in furnaces and for flame spread. In turn, particle formation and radiative energy transfer control the amount of smoke produced, which is important in fire detection and pollutant emission. In contrast to the oxidation of simple hydrocarbons, which is well understood, the detailed mechanisms for producing large hydrocarbons during combustion have not been established. The elucidation of chemical growth mechanisms continues to be one of the most challenging research problems in combustion science today.

While the chemical growth reactions play a key role in regard to the total amount of smoke produced, the actual structure of the smoke leaving the flame region is determined by a physical agglomeration process of particles sticking 
structure. The light extinction coefficient and light scattering coefficient, both of which affect the visibility through smoke, are not well characterized for agglomerate structures. The aerodynamic properties, which control smoke deposition in the respiratory tract, are also poorly characterized. Understanding the relationship between the geometric structure of an agglomerate and its properties is a key research topic in both aerosol research and condensed matter physics.

\section{Soot Formation}

This is the ninth year of a long-term study of the fundamental chemistry of soot formation, carried out jointly with the NBS Thermal Processes Division and also in collaboration with the Chemical Kinetics Division. Detailed flame structure measurements (species concentrations, temperature, and velocity) have been made in a laminar methane/air diffusion flame using a variety of laser-based optical techniques as well as mass spectrometric sampling. By using all of these data, the species profiles have been analyzed to determine production and destruction rates of intermediate hydrocarbons. The results show that acetylene plays the major role in surface growth on particles (as found in premixed flame studies). Furthermore, direct tests of proposed models of chemical growth can now be made which strongly suggest that a pathway involving vinyl radical addition to acetylene is the fastest route to form benzene. Experiments have also investigated fuel structure effects on chemical growth pathways by the addition of small amounts of ethylene, butadiene, and toluene to the base methane flame.

Recently the first quantitative $\mathrm{OH}$ concentration measurements have been made for a hydrocarbon diffusion flame. These results will enable us to determine the most rapid production pathways for forming vinyl and ethynyl radicals, which are thought to be key precursors in chemical growth, and to greatly expand our analysis of proposed mechanisms for soot formation. New results have also been obtained for detection of $\mathrm{H}, \mathrm{O}, \mathrm{C}, \mathrm{CH}, \mathrm{O}_{2}$, and $\mathrm{CO}$ by means of multiphoton ionization spectroscopy. Profiles of these species are being measured in our methane/air diffusion flame.

\section{Smoke Agglomerates}

A combined experimental and theoretical effort is focussed on the characterization of the optical properties of smoke agglomerates. The theoretical effort has consisted of generating simulated smoke agglomerates of various sizes and then computing the light scattered by the agglomerates using Rayleigh-Debye scattering theory. The most noteworthy result of this analysis is the prospect of inferring information about the concentration and size of the soot from the scattering measurements. It is shown that from the scattering measurements one can infer the overall size of the agglomerate, the size of the primary units in the agglomerate, the number of primary units in the agglomerate, and the number concentration of agglomerates.

To study the optical properties of smoke, a combined transmission cellreciprocal nephelometer has been developed. This device allows the simultaneous measurement of the extinction coefficient and the total scattering coefficient of smoke. These are the key quantities needed for studying radiation transport through a smoke cloud. From the difference of these two quantities, the absorption coefficient of smoke can be determined. 
The specific scattering and absorption coefficient for acetylene smoke are found to be $2.2 \mathrm{~m}^{2} / \mathrm{g}$ and $6.9 \mathrm{~m}^{2} / \mathrm{g}$, respectively. It was found that the smoke can be changed to a nearly spherical structure by exposing the smoke to a condensation/evaporation cycle. This "cloud processing" mechanism results in a doubling of the scattering coefficient while the absorption coefficient is unchanged. These results are consistent with the Berry-Percival theory regarding the optical properties of smoke clusters.

A Smoke Aging/Dilution chamber has been fabricated for use in large scale fire tests involving the furniture calorimeter. The facility allows rapid filling of a $1 \mathrm{~m}^{3}$ chamber within a few seconds with smoke collected just above the fire. The chamber walls can be heated up to $150^{\circ} \mathrm{C}$ allowing the matching of wall temperature to the smoke temperature as it rises in the stack.

Preliminary measurements have been performed regarding the effect of aging over a one hour period on the extinction coefficient of crude oil smoke for three wavelengths spanning the visible range. The results indicate that while the agglomerates increase in size during the aging, the specific extinction coefficient is not affected. An extensive series of smoke aging experiments are planned for the next year including the determination of the agglomeration coefficient, the change in the size distribution function, as well as optical properties measurements. These measurements will be performed both at ambient and elevated temperatures.

\section{Reports and Publications}

"Chemical Production Rates of Intermediate Hydrocarbons in a Methane/Air Diffusion Flame" by Miller, J.H., Mallard, W.G., and Smyth, K.C., Twenty-First Symposium (International) on Combustion 1057 (1986).

"Multiphoton Ionization Detection of $\mathrm{CH}$, Carbon Atoms, and $\mathrm{O}_{2}$ in Premixed Hydrocarbon Flames" by Tjossem, P.J.H. and Smyth, K.C.

"Concentrations and Production Rates of the Hydroxyl and Radical in a Laminar Methanel Air Diffusion Flame" by Miller, J.H., Tjossem, P.J.H., Hamins, A., and Smyth, K.C. submitted to Combustion and Flame.

"Methyl Radical Concentrations and Production Rates in a Laminar Methane/Air Diffusion Flame" by Miller, J.H., and Taylor, P.H., Combustion Science and Technology, 52, 139 (1987).

"Measurement and Rate Law Analysis of $\mathrm{D}_{2}$ Q-Branch Line Broadening Coefficients for Collisions with $\mathrm{D}_{2}$, $\mathrm{He}$, Ar, $\mathrm{H}_{2}$, and $\mathrm{CH}_{4}$ " by Smyth, K.C., Roscasco, G.J., and Hurst, W.S., J.Chem. Phys. , 87, 1001, (1987).

"Chemistry of Molecular Growth Processes in Flames" by Smyth, K.C. and Miller, J.H., Science, 236, 1540 (1987).

"Soot Particle Formation in Laminar Diffusion Flames" by Santoro, R.S. and Miller, J.H., Langmuir, 3, 244 (1987).

-"Smoke Production and Properties" by Mulholland, G.W., Society of Fire Protection Engineers Handbook, 1988. 
"Fractal Analysis of Soot Agglomerates" by Samson, R.J., Mulholland, G.W., and Gentry, J.W., Langmuir, 3, 272 (1987).

"Cluster Size Distribution for Free Molecular Agglomeration" by Mulholland, G.W., Samson, R.J., Mountain, R.D., and Ernst, M.H., Energy and Fuels (in press).

"The Effect of Scale on Smoke Emission" by Mulholland, G.W., Henzel, V., and Babrauskas, V., presented to 2nd International Fire Safety Science Symposium.

"Light Scattering from Simulated Smoke Agglomerates" by Mountain, R.D. and Mulholland, G.W., accepted for publication in Langmuir.

\section{Related Grants}

1. "Soot Dynamics in Flames", R.A. Dobbins, Brown University.

2. "Chemical Pathways to Soot Formation in Diffusion Flames", J.H. Miller, George Washington University.

3. "Soot Particle Formation and Destruction in Diffusion Flames", R.J. Santoro, Pennsylvania State University. (See p. 126 for description)

4. "Cooperative Research in the Kinetics of Agglomerate Formation", James W. Gentry, The University of Maryland.

5. "Fire Modeling", P.J. Pagni, University of California, Berkeley. 


\section{CENTER FOR FIRE RESEARCH \\ NATIONAL BUREAU OF STANDARDS \\ FY 88}

Institution: Brown University

Grant No: 60NANB6D0643

Grant Title: Soot Morphology in Flames

Principal Investigator: Professor R. A. Dobbins

Division of Engineering

Brown University

Providence, R. I. 02912

Other Professional Personnel: Dr. C. M. Megaridis

Post Doctoral Research

Investigator

NBS Scientific Officer: Dr. George W. Mulholland

Technical Abstract:

Introduction: An improved knowledge of soot properties has application to the use of smoke as an early detector of fire, to the improved prediction of the radiation properties of flames, and through the latter, to a better understanding of the spread of fires. The object of this study has been the determination of the morphological character of the soot particles in diffusion flames and the interpretation of this information in terms of the soot dynamic processes. To achieve this goal we have developed a thermophoretic sampling technique in which the sampling probe is rapidly inserted into the flame, held for a controlled brief interval (ca. $30-100$ ms), and withdrawn rapidly. The captured sample is then placed directly in the electron microscope for TEM examination without handing or transfer operations. The details of this technique have been reported(1,2). To quantitatively interpret the morphological observations we have developed a moment integral solution to the derosol dynamic equation with simultaneous particle inception, surface growth, and coagulative growth. Using this novel experimental method and modeling by the aerosol dynamic equation, we have made significant progress in understanding the production, transport, and oxidation and/or release of soot to the environment. These results are recapitulated briefly herein and are described in fuller detail in the reports and publications cited below.

1. Thermophoretic Sampling: The technique provides soot particle samples for the determination of the primary particle size of agglomerated chains. Primary particle size is a durable property which can provide valuable information on the history of the soot field. The state of agglomeration of the soot particle field can also be determined but agglomerate samples are fragile and are subject to damage during handling procedures. The variation 
of the particle morphology - primary particle size and extent of agglomeration - along a particle path line in the flame provides information on the particle field and the dynamic processes to which it is subject.

Our observations have been conducted mainly in the ethene coannular diffusion flame, and they confirm previous reports that the primary particles from any one point of origin within the flame are highly monodisperse. This result has important consequences in the modeling of the optical properties of the agglomerates. Optical observations do provide an accurate measure of the particle volume fraction which, when combined with the knowledge of the primary particle size, can yield both the primary particle number density and the total surface area per unit volume of space. We find values of surface area of 6 to $15 \mathrm{~cm} 2 / \mathrm{cm} 3$ typical of the annular region of the ethene diffusion flame.

The thermophoretic sampling technique provides excellent data on the primary particle diameter vs. height in the flame along a particle path line. The size information can be combined with data on the local gas velocity to yield specific surface growth rates of the soot particles. Our results(3) obtained using the flame velocity measurements of santoro et al.(R1) are shown in Figure 1 below. Our measurements are intermediate to the corresponding data by Santoro(R2) for the same flame and the data reported by Harris and Weiner(R3) in a premixed ethene flame that produces lower soot concentrations.

Along a given height in the ethene diffusion flame we find the maximum primary particle size at the radius of maximum particle volume fraction. Primary particles are smaller at the larger radii where the local temperature and oxygen concentration is higher.

The decrease in soot particle size with height in the upper portion of the nonsmoking ethene flame can be used to find the order of magnitude of the oxidation rates. We find the soot particle burnout rate to be $10-4 \mathrm{gm} / \mathrm{cm} 2 \mathrm{sec}$ in regions where the flame temperature ranges from 1500 to $1800 \mathrm{~K}$. Values of 10-8 to 10-3 gm/cm2 $\mathrm{sec}$ have been reported in the literature for this quantity which strongly depends on the particular combustion environment.

2. Soot Aerosol Dynamics: To interpret the experimental results we have formulated a bimodal integral solution to the aerosol dynamic equation (2-4). The experimental data are used to the maximum extent to quantify the processes which control the soot aerosol dynamics. Some results of this modeling are as follows:

-Soot inception is extremely intense in an annular region in the lowest portion of the ethene diffusion flame. In this region we infer particle inception rates of about $1 \times 10+16 \mathrm{~cm}^{-3 \mathrm{~s}^{-1}}$. These rates are consistent with previous estimations in various combustion environments.

-A criterion for the development of collisional growth is formulated that provides a test for the likehood of the presence or absence of agglomeration when the primary particle size and particle volume fraction are known. This test applied to our flame indicates that the presence of agglomerates is consistent with the observed primary particle size.

-The aerosol dynamic model shows that larger particles within the free molecular flow regime will cause more rapid agglomerative growth. This result can explain the anomalous lower values of the soot particle number 
concentration at the same radial location in the soot field where both the particle volume fraction and the mean particle size possess their maxima. In this region the intense surface growth explains the high particle volume fraction. The larger sizes cause a more intense agglomeration and reduce the local soot particle number concentration.

-The moments of the volume equivalent diameters of the agglomerates are controlled by diverse effects such as agglomerative and surface growth. Our observations indicate that the various moments have ratios that are well approximated by the values obtained under the self-preserving size distribution assumption.

3. Soot Morphological Development: The morphology of agglomerates on the path line corresponding to the maximum soot volume fraction has been studied in detail(3). We find that the primary particle size increases to a maximum just above the midpoint of the flame. Agglomerative growth is sustained throughout this region including into the upper portion of the flame where oxidation dominates. Oxidation reduces the primary particle size and burns the agglomerates completely at the top of the flame in the case of the nonsmoking flame. This description is displayed schematically in Fig. 2.

Only a limited statistical analysis of the agglomerates has been performed because of the cost of this type of analysis. At $z=15 \mathrm{~mm}$ in the nonsmoking ethene flame we find the fractal dimension to be 1.73 and 1.85 for the two samples which were analyzed. These values of the fractal dimension are consistent with the occurrence of agglomerative growth by cluster-cluster collision processes.

The investigation of a lightly sooting methane flame showed slight agglomeration and modest amounts of surface growth.

4. Optical Properties of Polydisperse Agglomerates: A description of the optical properties of agglomerates with an emphasis on polydispersity, i.e., variable chain length with monodisperse primary particles, has been formulated(5). This model provides the optical properties of interest in the prediction of radiative properties and interpretation of laser scattering experiments, as well as the prediction of agglomerate albedo and specific extinction. This model relies on the calculations by Felske et al.(R4) for monodisperse agglomerates. It also agrees with the model by Mountain and Mulholland (R5) in the limit of small ratio of radius of gyration to wavelength of light. Further comparisons of these methods with one another and with the available experimental data are being conducted.

\section{References:}

R1. Santoro, R. J., et al., Combustion Sci. and Tech. 53, 89, 1987.

R2. Santoro, R. J., Twentieth Fall Technical Meeting, Eastern Section, Paper 19, The Combustion Institute, 1987.

R3. Harris, S. J., and Weiner, A. M., Combustion Sci. and Tech. 31, 155, 1983. 
R4. Felske, J. D., et al., J. Quant. Spectrosc. Radiat. Transfer 35,447 , 1986.

R5. Mountain, R. D. and Mulholland, G. W., "Light scattering from Simulated Smoke Agglomerates", NBS manuscript submitted for publication, May 1988 .

Reports and Publications:

1. Dobbins, R. A. and Megaridis, C. M., "Morphology of Flame Generated Soot as Determined by Thermophoretic Sampling", Langmuir 3, 254, 1987.

2. Megaridis, C. M., "Thermophoretic Sampling and Soot Aerosol Dynamics of an Ethene Diffusion Flame", National Bureau of Standards Report, NBS-GCR-87-532, December 1987.

3. Megaridis, C. M. and Dobbins, R. A., "Soot Aerosol Dynamics in a Laminar Ethylene Diffusion Flame", to appear in the 22nd International Symposium on Combustion, 1988.

4. Megaridis, C. M., and Dobbins, R. A., "An Integral solution of the Aerosol Dynamic Equation Including Surface Growth Reactions", to appear in Combustion science and Technology.

5. Dobbins, R. A., and Megaridis, C. M., "Absorption and Scattering of Light by Flame Generated Polydisperse Agglomerates", manuscript in preparation.

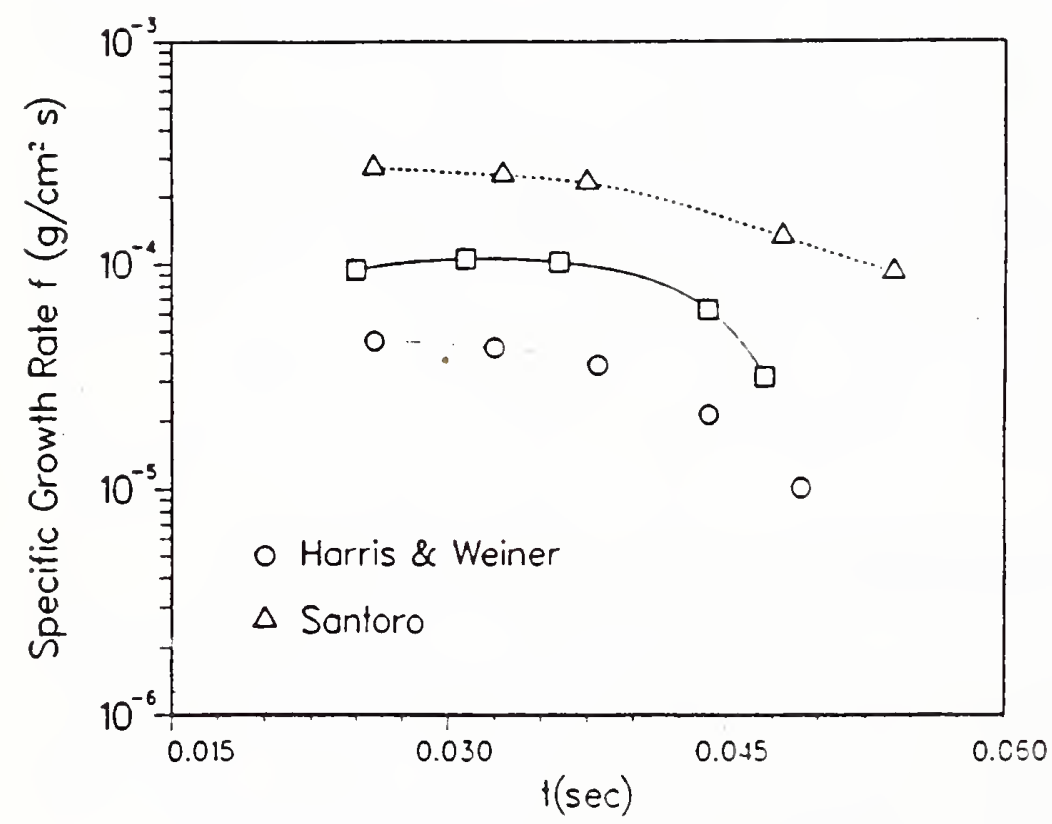

Fig.i specific soot surface growth rate

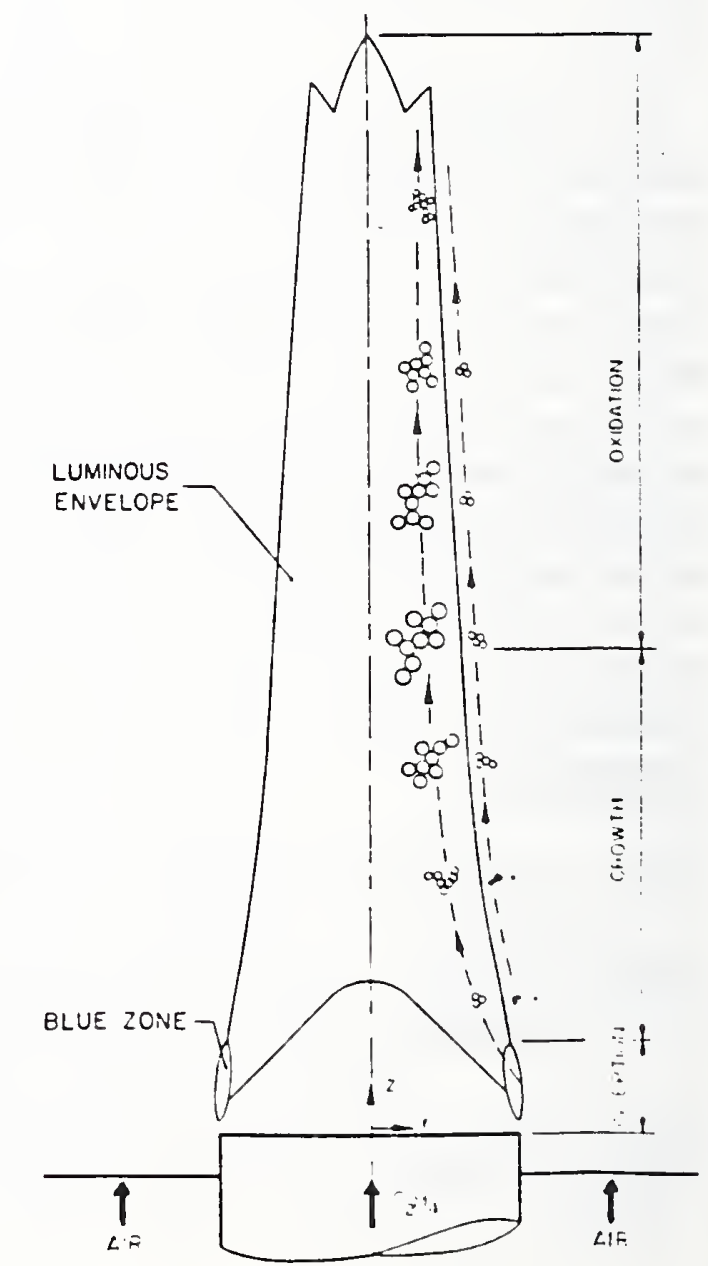

Fig.2 Soot morpholoay in a flame 


\section{CENTER FOR FIRE RESEARCH \\ NATIONAL BUREAU OF STANDARDS \\ FY 88}

Institution: The George Washington University

Grant Number: 60NANB6D0625

Grant Title: Chemical Pathways to Soot Formation in Diffusion Flames

Principal Investigator: Professor J. Houston Miller

Department of Chemistry

The George Washington University

Washington, DC 20052

(202) $994-7474$

Other Professional Personnel: Dr. Anthony Hamins, Postdoctoral Research Assoc. NBS Sclentific Officer: Dr. Kermit C. Smyth

\section{Technical Abstract \\ Introduction}

Chemical growth reactions which occur during hydrocarbon combustion lead to the formation of polycyclic aromatic hydrocarbons and soot particles. As a consequence, many pollutants and smoke may be emitted from flames. Soot is involved in determining energy transfer and the temperature field within the flame environment. It is now believed that the early steps of chemical growth, the soot inception phase, are rate-limiting and control the later stages of growth.

The chemical steps of molecular growth from small hydrocarbon molecules to aromatic species and eventually soot particles are not well established for flame conditions. From several studies performed in premixed flames [1-9] and in shock tubes [10-13], specific mechanisms have been proposed and predictions have been compared to measured species concentrations profiles. Available kinetic models successfully reproduce the experimental results for the major species but serious discrepancies arise for radicals and intermediate hydrocarbons $[8,9]$.

Despite these shortcomings of the theory, consensus is developing on the general scheme for this radical-based chemical growth mechanism. Fuel molecules are rapidly converted into a number of precursor species, predominately acetylene. After these pyrolyses reactions have run their course, the precursor species may react together to form larger hydrocarbon species, eventually leading to aromatic structures. Formation of subsequent aromatic rings has been suggested to proceed by continual addition of acetylene to a growing aromatic radical.

There have been few attempts to validate models for this subsequent $P A H$ growth chemistry through comparison with experimental data. An exception has been the recent work of Frenklach and Warnatz [10], who modelled the low pressure acetylene oxygen flame of Bockhorn et al. [13]. Although the agreement between peak concentrations and profile shapes is less than completely satisfactory in this work, it represents an exciting beginning to the application of detailed chemical models to studies of flame data. Unfortunately, such comparisons are not possible for more flame systems 
because of the lack of species-specific data on the concentrations of larger molecular systems.

Cooperative research by our group at George Washington University and workers at the National Bureau of Standards has concentrated on extending the study of early growth chemistry described above to a laminar, methane/air diffusion flame by making extensive concentration, temperature, and velocity measurements [15-20]. Because a laminar flame is a steady state system, changes in a species' concentration at given flame location balance one another. Therefore, concentration changes due to chemical processes are exactly the same magnitude as changes due to transport processes (convection and diffusion). We have exploited this relationship to calculate the net rates of chemical processes for intermediate hydrocarbons in our flame system [17] and can now compare these rates with the predictions of the mechanism for benzene formation presented above [20]. In last year's report, our experimental approach and results from the first few years of effort were summarized. The discussion which follows will concentrate on new results from the past year, organized according to research topics in last years proposal.

Scavenger Probe Sampling with Deuterium

The of the iodine scavenger probe which was used to profile the concentration of methyl radicals in our methane/air flame system has the disadvantage that iodine is somewhat corrosive. Recent literature suggested that a superior scavenging agent might be deuterium, which had been used to determine flame concentrations of $\mathrm{H}$ and 0 atoms and hydroxyl radicals [21]. We have modified our iodine scavenger probe for use with deuterium. Unfortunately, the results from our initial work with $D_{2}$ were unsatisfactory. In these experiments, it was found that profile signals at mass 3 (indicative of $\mathrm{H}$ atom scavenging) and 19 (indicative of hydroxyl radicals) showed evidence of isotopic scrambling within the probe. Profile signals at mass 17, thought to be indicative of methyl radical scavenging, had the same appearance as methane profiles. Although this might also be attributable to isotopic exchange within the probe, a more reasonable explanation would be interference from natural abundance ${ }^{13} \mathrm{C}$ (approximately 18 of ${ }^{12} \mathrm{C}$ ). Unfortunately, this interference will be present when applying deuterium scavenging to any hydrocarbon radical. A solution to the problem would be to try the experiment in a diffusion flame burning isotopically enriched ${ }^{12} \mathrm{C}$ methane. We are pursuing sources of funding to explore this experimental possibility.

\section{The Effect of Fuel Additives on the Formation of Aromatics}

The data set which is available from the GWU/CFR studies of a methane/air diffusion flame represents the most complete description of a hydrocarbon diffusion flame system. An appropriate extension to the methane flame studies would be a series of experiments in which small additions of materials to the methane fuel flow could be used to perturb the well-known flame structure. If the additions are small enough, their effects on flame temperature and convective velocities will be inconsequential, and it will not be necessary to repeat these difficult measurements. We have performed some of the hydrocarbon addition experiments (ethylene and toluene) in the last year. Addition of 1 to $3 \%$ ethylene to the fuel flow had very little effect on the concentrations of acetylene and benzene, the most prevalent intermediate hydrocarbons in the pure methane flame. However, the addition of one percent toluene to the fuel flow had dramatic effects on the flame structure: 
The concentration of benzene, phenyl acetylene, styrene, and naphthalene were much higher in the toluene-doped flame.

- Bibenzyl, benzaldehyde, and ethyl benzene were not observed in appreciable concentrations.

- Concentrations of acetylene and diacetylene were not appreciably different in the flame with toluene added.

A consistent picture for the fate of toluene in this diffusion flame emerges from this data. Low concentrations of benzaldehyde, ethyl benzene, and bibenzyl suggest that toluene decomposition via the benzyl radical is a minor pathway in this flame. In contrast, toluene is rapidly converted into significant concentrations of the phenyl radical via unimolecular processes or bimolecular reactions with radicals. If the phenyl were to decay further, dramatic increases in the concentrations of acetylene and diacetylene (from $\mathrm{C}_{4} \mathrm{H}_{3} \cdot$ ) would be observed. Instead, our data suggests that phenyl radicals are either rapidly converted into benzene (the dominant route) or react with acetylene to form either phenylacetylene or styrene. These species may then participate in subsequent growth processes which lead to the formation of the second aromatic ring, as indicated by the marked increase in the concentration of naphthalene.

Further work is necessary to clarify the evolution from one to two ring systems in our flame. In the case of the toluene addition experiments, this includes evaluating this proposed reaction sequence quantitatively through a determination of the production rates for species which might be involved in aromatic ring formation processes. Preliminary results show dramatic differences in the observed production rates for such important intermediates as phenylacetylene in the methane/toluene flame when compared to the pure methane flame. Additional experimental work is also required and will be discussed below.

It might be possible to distinguish between phenyl and benzyl radical based chemistries by repeating the addition experiments with fully deuterated toluene. If phenyl radical is formed quickly from toluene, and is rapidly converted into benzene, it should be possible to observe a signal indicative of the presence of $\mathrm{C}_{6} \mathrm{D}_{5} \mathrm{H}$ from the reaction of deuterated phenyl with $\mathrm{H}_{2}$ or $\mathrm{H}_{2} \mathrm{O}$. It may also be possible to follow the growth of the deuterated additive through detection of $\mathrm{C}_{6} \mathrm{D}_{5}-\mathrm{C}_{2} \mathrm{H}$ (via $\mathrm{R} 14$ ) and partially deuterated naphthalene (through R15-R17). We presently have on hand approximately $200 \mathrm{ml}$ of toluened6 and will soon try this experiment.

If it is correct to view the role of toluene in the methane flame as a source of phenyl radicals, it should be possible to add other species to the flame which might more directly perturb phenyl radical concentrations. One possibility is the addition of nitroso-benzene $\left(\mathrm{NO}-\mathrm{C}_{6} \mathrm{H}_{5}\right)$, which is often used as a phenyl precursor in kinetic studies of phenyl chemistry. The vapor pressure of this compound is relatively low (approximately one torr at $298 \mathrm{~K}$ ). However, the conversion of this compound into phenyl is nearly quantitative at $600 \mathrm{~K}$. It is expected that the concentration of phenyl in the pure methane flame is quite small (less than $10 \mathrm{ppm}$ ). Thus, addition of only one torr of nitroso-benzene should have dramatic effects on hydrocarbon growth chemistry. If necessary, this concentration could be boosted by heating the nitrosobenzene in the fuel line. 


\section{References}

1. J.D. Bittner and J.B. Howard, Eighteenth Symposium (International) on Combustion, p. 1105, The Combustion Institute, 1981.

2. J.D. Bittner and J.B. Howard, Particulate Carbon: Formation During Combustion (D.C. Siegla and G.W. Smith, Eds.), P. 109, Plenum Press, New York, 1981.

3. J.B. Howard and J.D. Bittner, Soot in Combustion Systems and its Toxic Properties (J. Lahaye and G. Prado, Eds.), P. 57, Plenum Press, New York, 1983.

4. J.D. Bittner, J.B. Howard, and H.B. Palmer, Soot in Combustion Systems and its Toxic Properties (J. Lahaye and G. Prado, Eds.), p. 95, Plenum Press, New York, 1983.

5. J.D. Bittner, D. Sc. Dissertation, Massachusetts Institute of Technology, 1981.

6. J.D. Bittner and J.B. Howard, Nineteenth Symposium (International) on Combustion, p. 211, The Combustion Institute, 1982.

7. J.A. Cole, J.D. Bittner, J.P. Longwe11, and J.B. Howard, Comb. \& Flame 56, 51 (1984).

8. R.J. Hennessy, C. Robinson, and D.B. Smith, Twenty-First Symposium (International) on Combustion, The Combustion Institute, 1986 (in press).

9. P.R. Westmoreland, J.B. Howard, and J.P. Longwell, Twenty-First Symposium (International) on Combustion, The Combustion Institute, 1986 (in press).

10. M. Frenklach and J. Warnatz, Comb. Sci.\& Tech. 51, 265 (1987).

11. T. Tanzawa and W.C. Gardiner, Jr., J. Phys. Chem. 84, 236 (1980).

12. M. Frenklach, D.W. Clary, W.C. Gardiner, Jr., and S.E. Stein, Twentieth Symposium (International) on Combustion, p. 887, The Combustion Institute, 1984.

13. M.B. Colket III, Twenty-First Symposium (International) on Combustion, The Combustion Institute, 1986 (in press).

14. H. Bockhorn, F. Fetting, and H.W. Wenz, Ber. Buns. Phys. Chem. 87, 1067 (1983).

15. K.C. Smyth, J.H. Miller, R.C. Dorfman, W.G. Mallard, and R.J. Santoro, Comb. \& Flame 62, 157 (1985).

16. J.H. Miller and P.H. Taylor, Comb. Sci.\& Tech. 52, 139 (1987).

17. J.H. Miller, W.G. Mallard, and K.C. Smyth, Twenty-First Symposium (International) on Combustion, The Combustion Institute, 1986, p. 1057.

18. K.C. Smyth and J.H. Miller, Science 236, 1540 (1987).

19. J.H. Miller, P.J.H. Tjossem, A. Hamins, and K.C. Smyth, manuscript in preparation.

20. A. Hamins and J.H. Miller, manuscript in preparation,

21. J.V. Volponi, W.J. McLean, and R.M. Fristrom, Combust. Flame 65, 243 (1986).

\section{Recent Publications}

"Mechanistic Studies of Toluene Destruction in Diffusion Flames." A. Hamins, D. T. Anderson, and J.H. Miller, Chem. Phys. Processes Comb. 1987, Paper \#4.

"Concentrations and Productions Rates of Hydroxyl Radicals in a Laminar Methane/Air Diffusion Flame." J.H. Miller, P.J.H. Tjossem, A. Hamins, and K.C. Smyth, manuscript in preparation. 
CENTER FOR FIRE RESEARCH

NATIONAL BUREAU OF STANDARDS

FY 88

Institution: The University of Maryland at College Park

Grant No.: COMM 70 NAN $85 \mathrm{HO} 0517$

Grant Title: Kinetics of Aggregate Formation

Principal Investigator: Dr. James W. Gentry

Department of Chemical Engineering

University of Maryland

College Park, MD 20742

Other Professional Personnel:

T. G. Cleary, Graduate

Research Assisitant

NBS Scientific Officer:

Dr. George W. Mulholland

Technical Abstract:

Introduction Soot agglomerates produced by the combustion of acetylene in a coannular diffusion burner are studied. The agglomerates consist of primary particles which have a mean diameter of about 30 nanometers. They appear to be attached in a random fashion yielding a tenuous non-compact structure. Single agglomerates may contain up to thousands of primary particles. The aerodynamic properties determined from particle motion and deposition can be related to the agglomerate structure. The structural properties and the distribution of sizes affects light scattering and absorption of the soot aerosol. Characterization of the aerosol stream from the burner provides a basis for the use of the burner in other studies and yields information about soot agglomerates in general.

Aerosol Generation The coannular diffusion burner has been described in detail by Santoro [1]. An integral part of the soot generating system is a chimney assembly designed to eliminate the effects of room air currents and to allow the operation of the burner at elevated pressure (up to $100 \mathrm{~cm}$ of water above atmospheric pressure). The chimney constructed of brass includes a viewing port for visual inspection of the flame. The output from the generator is stable day after day. By varying the fuel flow rate from lower to higher flow rate, the flame conditions change from non-sooting to visibly sooting conditions. Particles formed in both regions have been studied.

Primary Particles In the pre-sooting conditions, experimental measurements of the diffusion coefficients of the particles at different fuel flow rates indicate that the source is highly monodisperse with diffusion coefficients corresponding to spherical particles of 15-20 nanometers. At higher fuel flow rates particle size does not increase, but the number concentration does increase. The particle number concentration then drops off as the visible sooting point is approached. Measurements of mass and number concentration just below the visible sooting point yields an average volume equivalent diameter of 25 nanometers. Some agglomeration appears to be occuring. The unagglomerated primary particles have not been observed by electron microscopy 
yet, but individual primary particles can be sized from electron micrographs of the agglomerates.

Size Distribution of Clusters At the visible sooting point, the mass concentration of the aerosol increases by a factor of twenty above the presooting mass concentration. A multistage cascade impactor separates the soot particles by inertial effects. Data analysis yields a mass mean diameter and standard deviation corresponding to spherical unit density particles with the same inertia as the agglomerates. From the visible sooting point to a highly sooting flame, the mass mean diameter given by the impactor varies from 0.4 to greater than 5 micrometers.

A sample of the soot, with the burner operating at the vislible sooting point, was collected on transmission electron micrograph grids using a thermal precipitator. The thermal precipitator collects the particles on a grid by a force field driving particles to a surface due to a temperature gradient that the aerosol stream passes through. Three different magnifications were used when performing size distribution analysis. The size was defined as the longest length times the width of the cluster, then the square root of the productis taken. Figure one is a histogram of the results. Each calculated size falls into a size range and over 650 particles were measured. At the lowere magnification, only larger clusters were measured in order to obtain better statistics of the larger clusters with out loking at cluster sizes where significant numbers of clusters were counted at higher magnification. Using the results of fractal analysis previously performed [2], the relationship between the number of primaries and the size of the cluster (as defined above) allows for the mass distribution of the sample to be calculated. Figure 2 is a histogram of the mass distribution of the collected soot.

Cluster Friction Coefficient Individual clusters were examined to determine how the agglomerate structure affects the friction coefficient of the cluster. Odumade in his $\mathrm{PhD}$ study at Minnesota separated clusters by electrical mobility [3]. With electron microscopy the number of primary particles and the mean primary particle size for a cluster was calculated for separate clusters. Samson has collected samples of soot classsified by electrical mobility. Electron micrographic analysis of the clusters yields the number of primary particles in each cluster. Figure 3 is a plot of both odumade's and Samson's data. The volume equivalent sphere diameter of the cluster is plooted against the diameter of a sphere with the same friction coefficient as the cluster. Solid lines are theoretical curves. Further work is being performed to extend the range of the measured values.

Conclusions The primary particle size in the pre-sooting range was measured as a function of acetylene flow rate, and was found to be fairly constant in diameter. The size distribution analysis by impaction and electron microscopy was performed. Experimental measurements of cluster friction coefficients are being compared to theoretical models based on rarefied gas dynamics.

\section{$\underline{\text { References }}$}

1. Santoro, R. J. et al., Combustion and Flame, 51, 203-218 (1983). 
2. Samson, R., M.S. Thesis, University of Maryland (1986).

3. Odumade, O.A., Ph.D. Dissertation, University of Minn. (1983).

\section{Reports and Papers}

1. Cleary, T., R. Samson, G. Mulholland, and J.W. Gentry, "The Relationship Between Structure and Aerodynamic Measurements for Clusters", Proceeding G Ae F Conference, Hanover, September 1987

2. Gentry, J. W., and T. Cleary, "Diffusion Coefficients of Clusters", Annual Conference on Fire Research, NBS-Gaithersburg, MD, 1987

3. Gentry, J.W., G. Mulholland, R. Samson, and T. Cleary, "Methodology for Fractal Analysis of Combustion Aerosols and Particle Clusters", Accepted by Journal of Aerosol Science and Technology, 1988

4. Cleary, T., G. Mulholland, and J.W. Gentry, "The Experimental Measurements of Clusters Formed From Soot Agglomerates Near the Sooting Limit", Paper to be presented at 12 th International Conference on Atmospheric Aerosols and Nucleation, Vienna, Austria, 1988

5. Cleary, T., G. Mulholland, J.W. Gentry, "Correlations of Aerodynamic Diameters and Diffusion Coefficients with Geometric Structure for Soot Agglomerates", Paper submitted for AAAR Annual Meeting, Research Triangle, NC. 1988

Figure 1.

\section{Size Distribution of Soot Agglomerates}

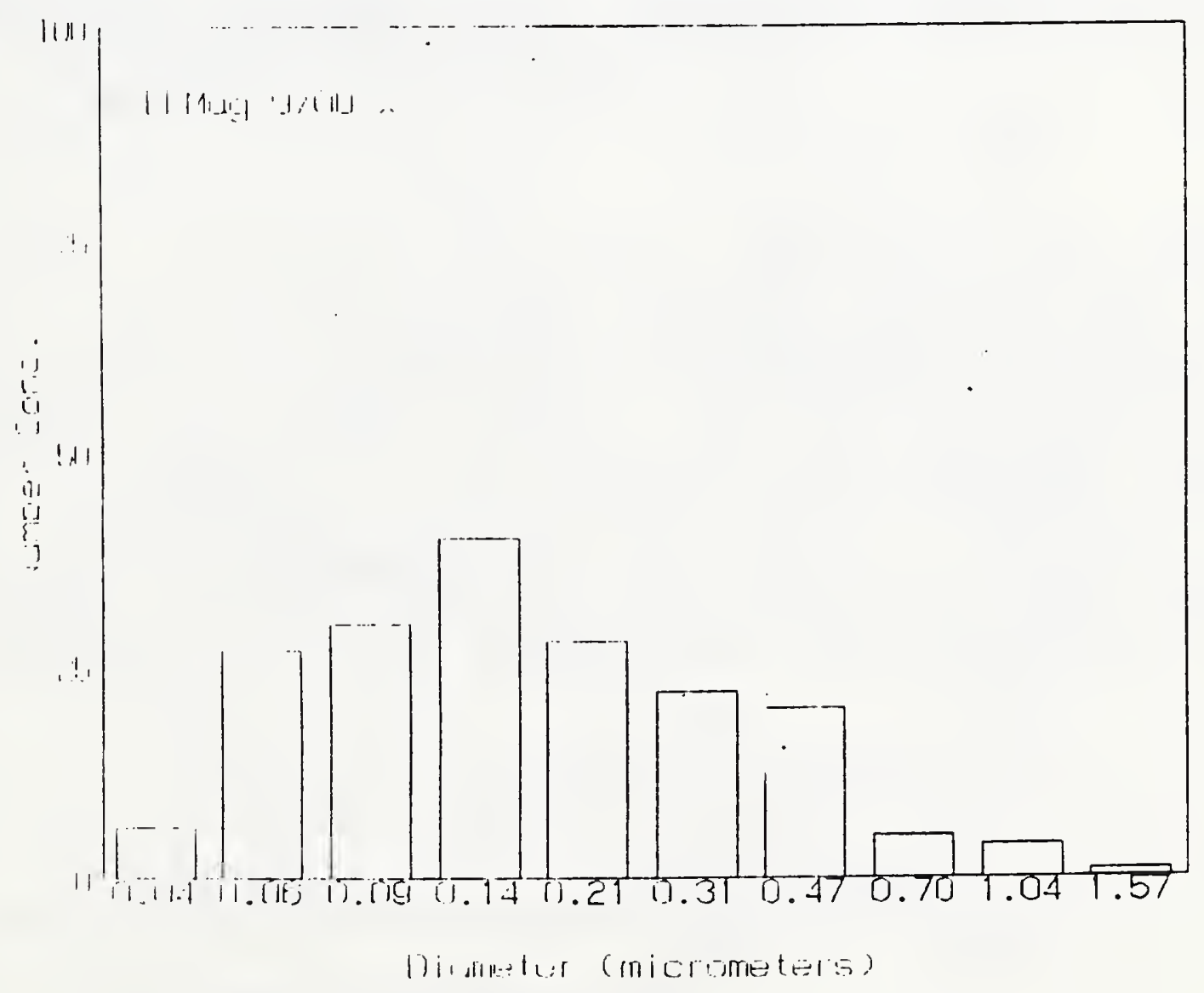


Figure 2 .

Size Distribution of Soot Agglomerates

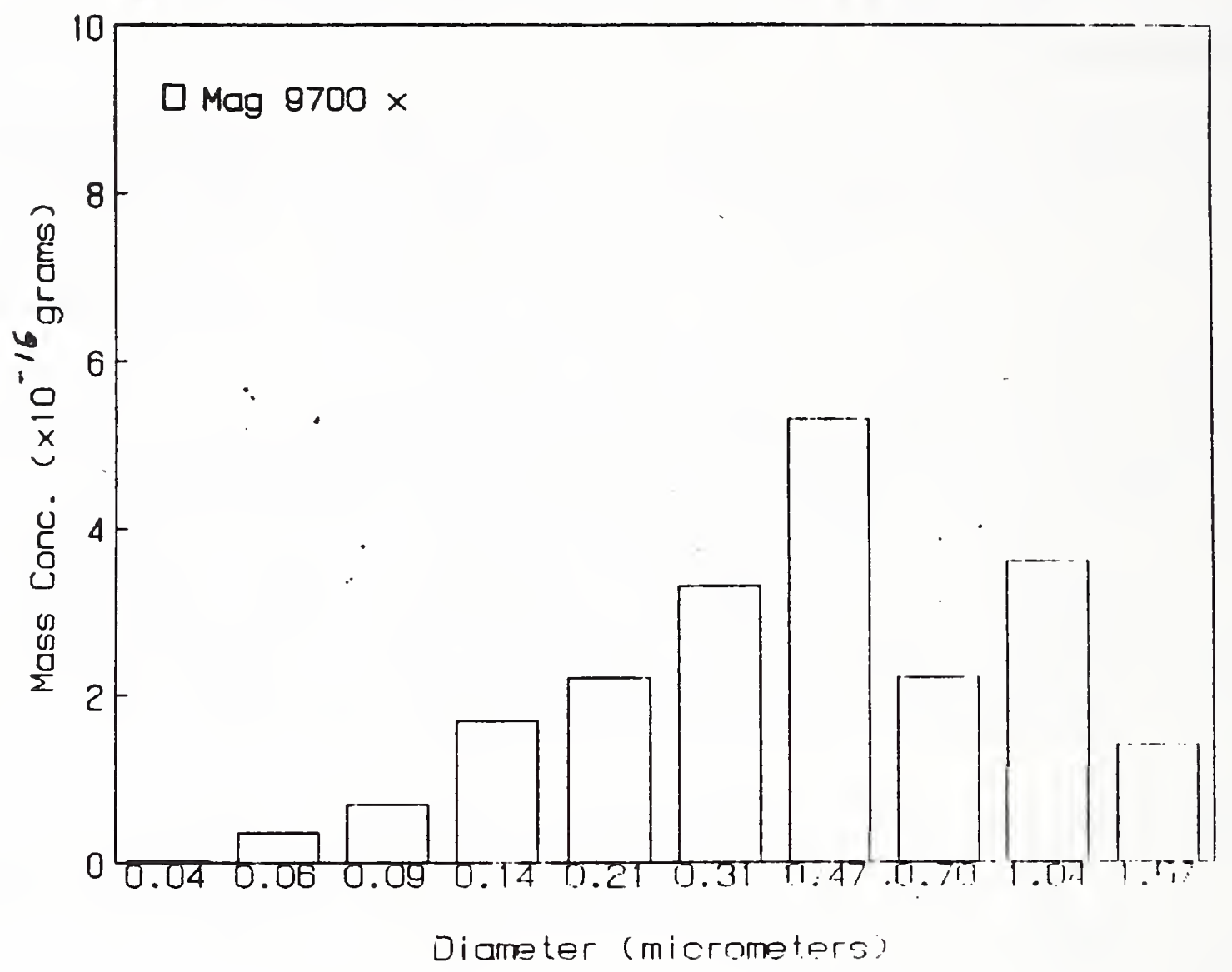

Figure 3. Dve is the volume equvalent sphere size of the cluster, Fc is the friction coefficient.

\section{Dve of Cluster vs Dp Sphere with same Fc Friction coefficient for data points from electrical mobility measurements}

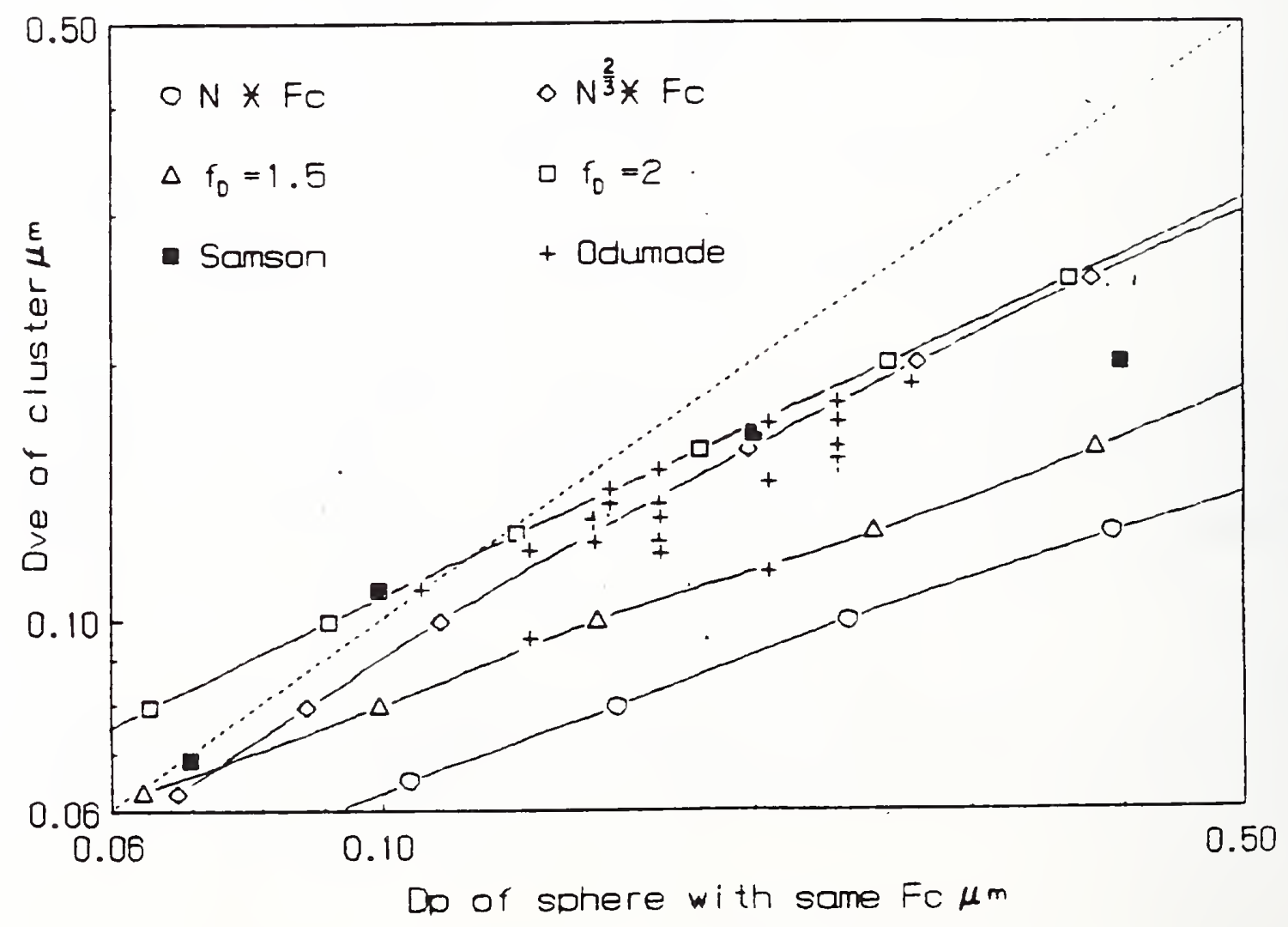




\section{CENTER FOR FIRE RESEARCH NATIONAL BUREAU OF STANDARDS \\ FY 87}

Institution: University of California, Berkeley

Grant No.: $\quad$ 6ONANB8D0848

Grant Title: Fire Modeling

Principal Investigator: Professor Patrick J. Pagni

Vice Chairman-Graduate Study

Mechanical Engineering Department

University of California

Berkeley, CA 94720

Telephone: (415)642-0729

other Professional Personnel: Nabil Elkouh (Ph.D Candidate) Joseph Viray (M.S. Candidate)

NBS Scientific Officer: Dr. Howard Baum

\section{Technical Abstract:}

The overall goal of this project is to develop chemical, physical and mathematical models of the detailed combustion phenomena which control a fire's growth. our emphasis recently has been on measurement and prediction of the soot volume fraction, $f_{v}$, in a variety of flames, on window breaking in compartment fires and on an overview of fire physics.

Fire Radiation:

In flame radiation, the most important and least known parameter is $f_{v}$, the soot volume fraction, defined as the volume occupied by soot per unit of flame volume. Previous summaries have described a multi-wavelength laser extinction technique for in-situ measurement of the local flame $f_{v \prime}$ in free and forced boundary layer diffusion flames(1), pool fires(2) and premixed flames $(3,4)$. Detailed experimental comparisons with alternative singlewavelength scattering/extinction measurement methods have been reported $(3,4)$. Limited data on small pool fire mean soot volume fractions suggest $f_{v}$ scales with optical thickness, $k L$, as

$$
\begin{array}{lll}
f_{V} / f_{V_{\text {max }}}=1.5(k L)^{1 / 3} & , k L \leqslant 0.3 \\
f_{V} / f_{V \text { max }}=1 & , . k L \geqslant 0.3
\end{array}
$$


If this correlation holds for all fuels, it will permit scaling of the mean $f_{v}$ in pool fires of any size from laboratory scale measured $f_{v}(2)$.

\section{Window Cracking in Fires:}

Following Professor Emmons' description of window breaking, we developed an estimate of the typical increase in window temperature required to break it(5). An exposed window heats and expands placing its cooler shaded edge in tension until it cracks at a small defect, usually at the top inner edge. Glass can only stand a small tensile stress, $\sigma \sim 4 \times 10^{7} \mathrm{~N} / \mathrm{m}^{2}(\sim 6000$. Psi) . The stretch modulus for glass is $E=7.8 \times 10^{10} \mathrm{~N} / \mathrm{m}^{2}\left(1.1 \times 10^{7} \mathrm{psi}\right)$. So the strain, $\epsilon=\sigma / E$, required to crack the glass is, $\epsilon \sim 0.05 \%$ - The temperature rise needed to produce that strain is $\Delta T=t / a$ $\sim 58^{\circ} \mathrm{C}\left(104^{\circ} \mathrm{F}\right)$, since the coefficient of linear thermal expansion for glass is $\alpha=9.2 \times 10^{-6} \cdot c^{-1}\left(5.1 \times 10^{-6} \cdot \mathrm{F}^{-1}\right)$. These results appear to be independent of scale. So compartment modelers may assume windows act as vents after the glass reaches $T \sim 80^{\circ} \mathrm{C}$. The energy transport between flame, smoke layer and glass remains to be modeled.

\section{Table 1. FIRE PHYSICS}

\section{FUUID DYNAMICS}

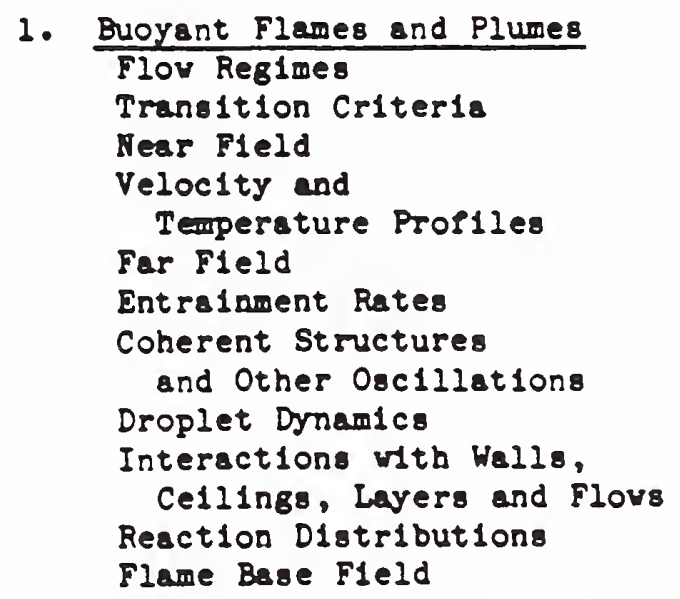

2. Flows Within Compartments

Celilng Layer Formation Two-Layer Temperatures Extended Fire Plumes Celling Jets

Strat1flcation Corridors Mixing

3. Flovs at Openings

Doors

Windows

Celling Holes

Multiple Openings

HVAC Systems

Mixing

4. Nondimensionalization
II. DIFFUSION FLAMES
1. Pyrolys18 Rates/Flame Shapes Laminar Boundery Layers Free, Forced and Mixed Burning distinct from Pyrolysing Heat Relense Rate Databsses Turbulent Boundary Lavers Free, Forced and Muxed Radiant Augmentation Blockage Effects L1quid and Solld Pools Walls, Cellings and Corners Cribs, Carpets and Furniture Charring Melting

2. Excess Pyrolyzate

Production

Composition Accumulation and Flamability Limits

3. Flame Radiation Temperature, Geometry and Composition Effects Heat Release Rate Fraction Wavelength Dependence Soot as \% or Fuel Carbon Soot Volume Fraction Messurement Correlations Analyses

4. Nondimensionalization 
Table 1 FIRE PHYSICS continued

III. FLANE SPREAD

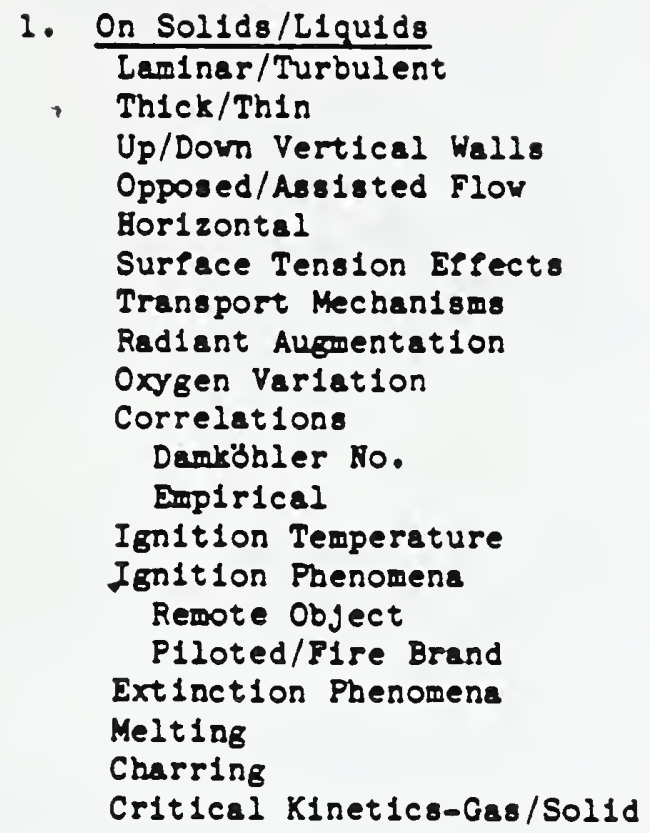

1. On Sol1ds/L1quids Laminar/Turbulent

- Thick/Thin

Up/Down Vertical Walls

Opposed/Assisted Flow

Horlzontal

Surface Tension Effects

Transport Mechanisms

Radiant Augmentation

Oxygen Varlation

Correlat1ons

Damröbler Ko.

Empirical

Ignition Temperature

Ignition Phenomena

Remote Object

Plloted/Fire Brand

Extinction Pbenomens

Melting

Charring

Critlcal Kinetics-Gas/Sol1d

2. In Sol1ds (Smoldering)

Porous Media Flov

Beat and Mass Transport

Counter-Current (Difrusion)

Co-Current (Premixed)

Self-heating to Igaltion

Surface Reactions

3. In Gases

Deflagrations/Detonetions

Consined/Unconsined

Pure Mixtures

Vit1sted Mixtures

Celling Layer Limits and Propagation Speeds

Ignition/Extinction

Stratification

4. Grouth Models

Exponent1al/Power Law

Doubling Time

Flre Growth Rate Parameter

Growth Classification

Intensity Classification

Threet Varlables

Escape Time

5. Nondimensionalization
IV. COMPARTMENT MODELING

1. Heat Transfer

Conduction Losses

Thermal Inertia

Convection Losses

Convection Between Layers

Flame Impingement

Smoke Layer

Visibility

Rediation

Surface Radiant Exchange

Accurately Described

Approximations

Radiat1on Rate Pool

2. Pyrolysis and Burning Rates

Ventilation Control

V1t1ation

Radiat1ve Feedbeck

3. Growth to Flashover

Vent1lat1on

Free, Forced, Hone

Pressure

Characteristic Times

Temperature Fleld H1story

Species Field History

Computetion

Zone

Field

Scale Effects

Stratification

Osc1llations

4. Flashover

Definitions

Criteria

Approximetions

Stabllity Analyses

Windows Bresking

Venting

Backdrefts

A15 Flames

5. F1re Spread to Adjacent Spaces

Postflashover Intensity

Flame Helghts Out Windows

Flame Propagation and Lengths

along Adjacent Cellings

Multiple Rooms

6. Nondimensionalization

Fire Physics Overview:

Fire physics is defined as the study of the physical mechanisms by which fire is initiated, grows throughout a system, and is extinguished. An inclusive list of topics within each of four broad categories: I.) Fluid Mechanics, II.) Diffusion Flames, III.) Flame Spread and IV.) Compartment Modeling, presented in Ref. 5 is given in Table 1. 
other studies related to fire are underway at Berkeley with separate support in the areas of smoldering(6) and compartment fire characteristic times(7). A invited review paper was presented at the Second International symposium on Fire Safety science. We are assisting the publications Committee with the proceedings of the Second International symposium(8). During this grant period I also attended the Tenth Meeting of the United States-Japan Conference on Development and Utilization of Natural Resources (UJNR) Panel on Fire Research and Safety in Tsukuba, Japan in June 1988. We are looking forward to hosting the Eleventh Panel Meeting here at the University of California at Berkeley in October 1989.

Reports and Papers:

1. J.A. Ang, P.J. Pagni, T.G. Mataga, J.M. Margle and V.J. Lyons, "Temperature and Velocity Profiles in Sooting Free convection

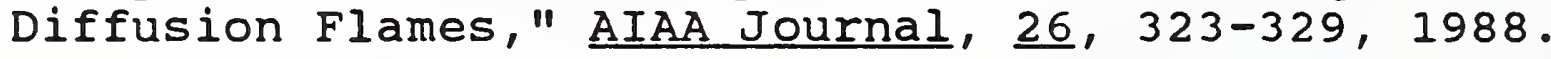

2. S. Bard and P.J. Pagni, "Spatial Variation of soot Volume Fractions in Pool Fire Diffusion Flames," Fire Safety science - Proceedings of the First International Symposium, pp. 361369, Hemisphere Publishing Co., New York (1986).

3. V.J. Lyons and P.J. Pagni, "A Comparison of Optical Measurements of soot in Premixed Propane/Oxygen Flames," Paper No. 46, Central section of the Combustion Institute, 1988 Spring Meeting, Indianapolis.

4. V.J. Lyons and P.J. Pagni, "Optical measurements of soot and Temperature Profiles in Premixed Propane-oxygen Flames," Poster Paper No. 40, p. 135, in the Book of Abstracts of the Twenty-second Symposium (International) on combustion, Seattle, Washington, August 14-19, 1988.

5. P.J. Pagni, "Fire Physics - Promises, Problems and Progress," Fire Safety science - Proceedings of the second International Symposium, pp. 49-66, Hemisphere Publishing Co., New York (1989).

6. J.L. Newhall, A.C. Fernandez-Pello and P.J. Pagni, "Experimental observations of the Effect of Buoyancy on Cocurrent Smoldering," Paper No. 88-50, Western section of the Combustion Institute, 1988 spring Meeting, salt Lake city, Utah.

7. P.J. Pagni, N.J. Alvares and K.L. Foote, "Defining Characteristic Times in Forced Ventilation Enclosure Fires," Mathematical Modeling of Fires - STP 983, J.R. Mehaffey, ed., pp. 68-82, American Society for Testing and Materials, Philadelphia (1988).

8.- T. Wakamatsu, Y. Hasemi, A. Sekizawa, P.G. Seeger, P.J. Pagni, and C.E. Grant, eds., Fire safety Science - Proceedings of the Second International symposium, Hemisphere Publishing Co., New York, 1989. 
PART I. Basic Research and Tools for "Engineered" Fire Safety (In-House Projects and Associated Grants Funded by NIST)

C. COMPARTMENT FIRE MODELING 



\section{CENTER FOR FIRE RESEARCH \\ PRIORITY PROJECT - 1988 \\ WALL FIRE GROWTH IN ENCLOSURES}

\section{Professional Personnel}

K. Steckler, Project leader

H. Mitler, Physicist

W. Davis, Physicist

M. Harkleroad, Physicist

\section{Project Objective}

To develop and experimentally validate algorithms which predict the behavior of a burning wall in a room and which are usable in a zone-type compartmentfire model.

\section{$\underline{\text { Scope }}$}

This project addresses the flammability aspect of wall fires in enclosures. Both theoretical and experimental efforts are involved in developing and validating algorithms for predicting the pyrolysis rates per unit area and the flame spread rates of these materials. The algorithms are then embedded in FIRST, a mathematical compartment-fire zone model which provides for interaction between the burning wall and the environment it creates within the enclosure.

\section{Technical Accomplishments}

\section{Reduced-Scale Experiments}

The experiments conducted during the past year were designed to study the pyrolysis rate of a fully-ignited fixed-area vertical surface in a reducedscale $(1.0 \times 1.0 \times 0.75 \mathrm{~m})$ enclosure. Flame spread effects were purposely minimized. The experimental results provide a data base with which to compare the results of the theoretical model (described below) for the pyrolysis rate of a fixed-area wall fire in an enclosure. Since the theoretical effort has focused on non-charring, homogeneous materials, the fuel in each experiment was a $0.18 \times 0.73 \times 0.025 \mathrm{~m}$ slab of PMMA. The experimental results include the pyrolysis rate of the wall panel; incident radiant flux; oxygen, carbon dioxide, and carbon monoxide levels in the upper gas layer; oxygen level in the lower gas layer; and gas temperatures ... all as a function of time. The sole independent variable in the experiments was the width of the door opening to the compartment. Door widths ranging from 2.6 to 100 percent of the room width produced a wide range of ventilation conditions and pyrolysis rates.

\section{Pyrolysis Model}

\section{Description}

A steady-state model was developed to predict the pyrolysis rate of a noncharring, homogeneous material in a vertical orientation, given the dimensions of the pyrolyzing zone, material properties of the wall material, some 
properties of the flame and plume, incident external radiant flux, and oxygen level in the adjacent gas layer. The pyrolysis rate, $\dot{m} "$, is obtained from the steady-state energy balance at the surface of the material;

$$
\dot{\mathrm{m}}^{\prime \prime} \mathrm{H}_{\mathrm{v}}=\alpha_{\mathrm{w}} \phi_{\mathrm{in}}+\phi_{\mathrm{c}}-\phi_{\mathrm{rr}}
$$

where $H_{v}$ is the effective heat of vaporization of the wall material, $\alpha_{w}$ is the absorptivity of the wall surface, $\phi_{i n}$ the incident radiant flux, $\phi_{c}$ the convective flux, and $\phi_{\mathrm{rr}}$ the reradiation flux from the surface. The incident radiant flux is the sum of the radiant flux from the flame and the external flux from surrounding hot walls and/or hot gases. Since the radiation from the flame depends on the mean absorption coefficient, a significant result of the current work is the finding that this coefficient varies with height, $z$, and it does so in a simple way (i.e. linear in $1 / z$ ). The convective flux is expressed in terms of the spalding mass transfer number. The reradiation flux, which varies as the fourth power of the surface temperature, is formulated in terms of an Arrhenius expression to account for variations of surface temperature with pyrolysis rate. More complete details of the model and its derivation have been reported by Mitler [1,2].

\section{Comparison with Experimental Data}

The model, as described above, applies in the case of uniform conditions (i.e. external flux, oxygen level, and gas temperature). A wall fire within a compartment, however, produces a stratified environment which, in turn, establishes local conditions at the wall that vary with position. In the context of a two-layer zone model, the pyrolyzing wall panel can be viewed as the sum of two pieces; one in the upper layer and the other in the lower layer. Each piece is exposed to the uniform conditions characteristic of the layer in which it is located. The pyrolysis model can then be applied to each piece independently.

This approach was used to predict the pyrolysis rates measured during the reduced-scale PMMA wall-fire experiments. The measured fluxes, oxygen concentrations, and gas temperatures were manipulated to produce representative average values for each layer. These values were then input to the pyrolysis model and the pyrolysis rate calculated. Figure 1 shows the calculated and experimental results for a compartment with a door width of $0.33 \mathrm{~m}$. Results are displayed for the period beginning at the time of complete-area involvement and ending when the sample collapsed. Figure 2 summarizes the results obtained for the entire series of door widths. Discrepancies between experimental and calculated values are believed to be connected to the downward flow of melted material along the face on the burning panel. Molten flows were observed, to some degree, in each of the experiments.

\section{Insertion of Pyrolysis Model into FIRST}

FIRST, an NBS compartment-fire model, was chosen to be the testbed for the wall-fire-growth algorithm. The immediate goal of this task is to predict the results of the reduced-scale wall-fire experiments, given the properties and 
geometries of the fuel and the enclosure. Unlike the calculations described above, in which the environmental conditions (external flux, etc.) were input parameters, FIRST will calculate these conditions and the pyrolysis rate simultaneously.

The pyrolysis madel has been inserted into FIRST. Algorithms for calculating wall-plume mass and energy transfers, external radiant flux to the wall, and floor heating also have been inserted. Debugging is underway.

\section{Upward Flame Spread and Burnout Model}

A purely numerical model was developed to predict the upward spread rate of a pyrolysis front on a vertical slab or wall that has been ignited at the bottom. One of the advantages of a numerical approach is that heating fluxes which vary with time and position along the surface of the slab can be taken into account in a straightforward manner.

The first step is to divide the height of the slab into a series of equallyspaced points or nodes. At each node, the surface temperature is determined from an energy balance at the node. This balance is between energy gained from the wall flame and hot gases and walls of the enclosure, and energy lost due to radiation and conduction from the surface of the slab. It is assumed that the pyrolysis front reaches a particular node when the surface temperature of the node reaches the critical temperature, $\mathrm{T}_{i \mathrm{~g}}$, of the wall material. Once ignited, the pyrolysis model (described above) is activated and the regression rate and burnout front can be determined.

The principal assumptions are that there is a well-defined $\mathrm{T}_{\text {ig }}$, a welldefined heat of vaporization, no diffusion of heat parallel to the surface, and no charring or melting. Additional information is available in a report by Mitler [3].

\section{Reports and Publications}

1. Mitler, H.E., "Algorithm for the Mass Loss Rate of a Burning Wall", IAFSS, Tokyo, Japan, June 1988 .

2. Mitler, H.E., "Algorithm for the Mass Loss Rate of a Burning Wall", NBSIR 87-3682, December 1987.

3. Mitler, H.E., "Upward Spread Algorithm", NBSIR in review.

\section{Related Grants}

Prediction of Fire Dynamics, J. deRis and R. Alpert, Factory Mutual Research Corporation. (See page 31 for description) 


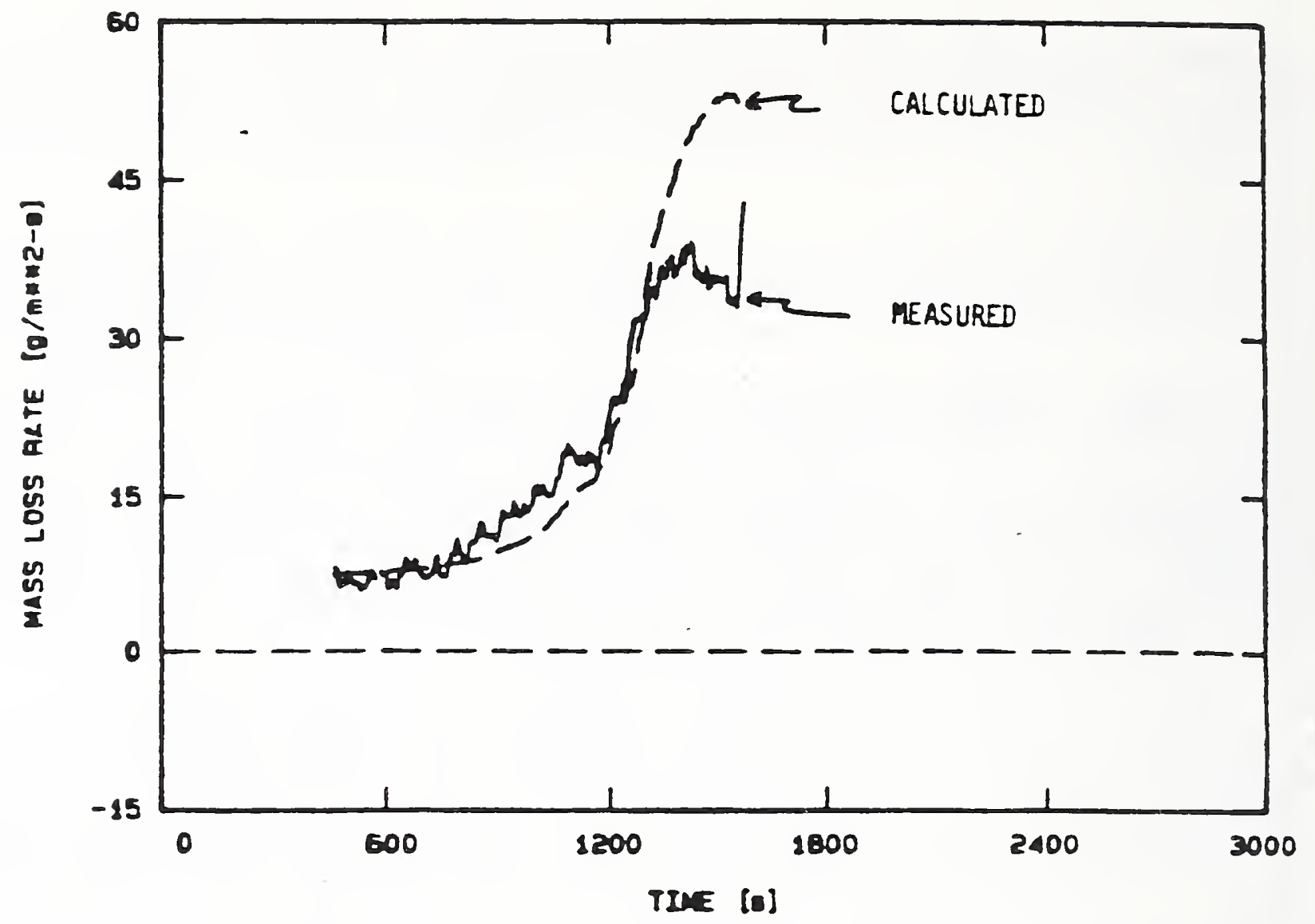

Figure 1. Comparison of calculated and measured mass loss rates for a PMA wall burning in a reduced-scale enclosure with a 0.33 mide doorway opening.

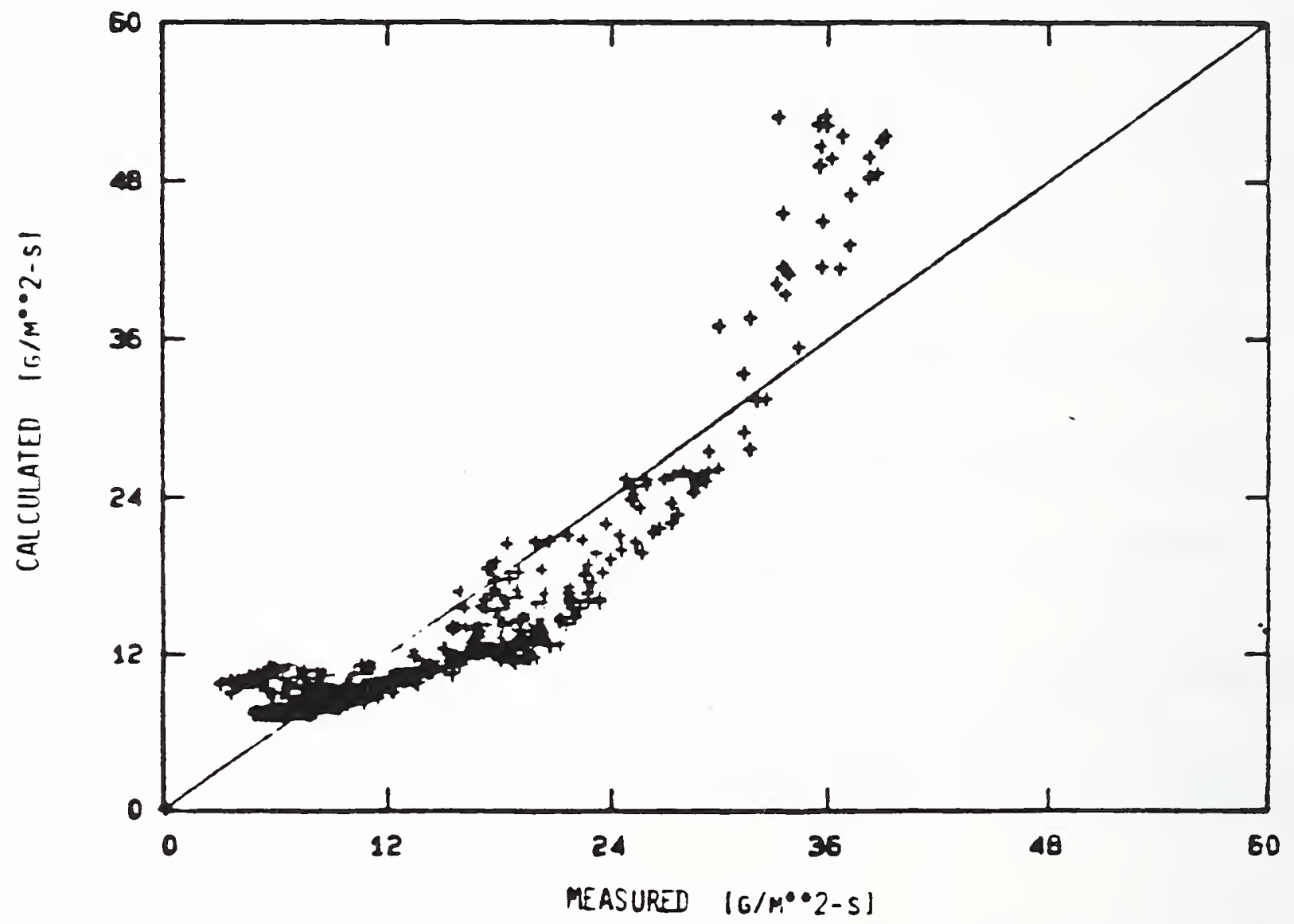

Figure 2. Comparison of calculated and mersured mass loss rates of PMA walls burning in a reduced-scale enclosure with variable door widths. 
CENTER FOR FIRE RESEARCH

PRIORITY PROJECT - 1988

\section{CONSOLIDATED COMPARTMENT FIRE MODEL}

\section{Professional Personnel}

Leonard Y. Cooper, Head, Building Fire Modeling Group and Project Leader John H. Klote, Mechanical Engineer

Glenn P. Forney, Computer Scientist

William Davis, Physicist

Daniel Alvord, Computer Specialist

Duy Q. Duong, Guest Worker (National Building Technology Center, Australia)

\section{Program Objectives}

Develop a comprehensive fire model computer code to simulate all physical and chemical aspects of fire in complex structures.

\section{$\underline{\text { Scope }}$}

The work of the project focuses mainly on the development of the Consolidated Compartment Fire Model (CCFM) computer code which consolidates past progress in zone-type compartment fire modeling, and which allows readily for integration of future advances with the greatest possible flexibility. It is envisioned that the project will lead to a series of well-documented, userfriendly, and numerically-robust CCFM products. These would be versatile in the sense that they would provide a capability of analyzing a particular compartment fire problem by using any one of a range of physical-phenomenamodeling sophistication, from the most basic to the most comprehensive. One final goal of the work is a Reference Fire Model (RFM) which, within the context of the zone-fire-model concept, would provide the best possible mathematical simulation of the fire environment. The project includes the effort of developing mathematical submodels of the separate compartment fire phenomena to be simulated. In this, concise algorithms and associated modular computer subroutines are developed which are readily useable in zone-type compartment fire model computer codes, in general, and in the CCFM/RFM, in particular.

\section{Technical Accomplishments}

The Consolidated Compartment Fire Model/Reference Fire Model (CCFM/RFM) computer code development process was initiated by establishing a generic CCFM equation set. This and other preliminary CCFM ideas were tested on the computer model, CCFM.HOLE, which simulates the fire environment in a single room with a hole of arbitrary area and elevation. Work then moved to multiroom model development. This has led to a prototype, multi-room code, called CCFM.VENTS which is now being completed and which we expect to document and release this coming year. This first-stage code involves a basic model formulation and code structure that allows for the required CCFM/RFM future growth flexibility. At this time the code is supported by a preliminary-draft user guide. Although it is being developed and tested on the NIST mainframe CYBER computer, the code runs also at comparable speeds on personal-computer 
hardware. Features of CCFM.VENTS include: simulations of concentrations of oxygen and an arbitrary number of other products of combustion; robust numerics; unforced vent flows under both low- and high-cross-vent pressure difference conditions; forced ventilation via simple fan/duct systems; and wind effects. Finally, the code uses the simplest possible, point-sourceplume, smoke-filling fire physics in the rooms-of-fire-origin and a very simple heat transfer calculation there and in other spaces. Throughout the course of the year, progress has been made on developing new and improved submodel algorithms and subroutines. These will be added eventually to future CCFM/RFM products.

\section{Funding from Other Agencies/Institutions}

In addition to CFR Priority Project funding the above activities were carried out with support from the following Other Agency funding:

Sponsor: Department of the Navy, Naval Sea Systems Command Title: Evaluation of Smoke Control on Navy Ships

\section{Reports and Publications}

"A Plan for the Development of the Generic Framework and Associated Computer Software for a Consolidated Compartment Fire Model Computer Code," G.P. Forney and L.Y. Cooper, NBSIR 86-3500, Jan. 1987.

"A Program for the Development of a Benchmark Compartment Fire Model Computer Code," L.Y. Cooper, J.A. Rockett, H.E. Mitler and D.W. Stroup, Special Technical Publication (STP) 983, pp 116-127, American Society for Testing and Materials, Philidelphia, PA (1988).

"The Compartment Fire-Generated Environment and Smoke Filling," L.Y. Cooper, presented at the International Symposium and Roundtable on Fire Protection Engineering Methods, Sept. 13-16, 1988; Section 2/Chapter 7 of The SFPE Handbook of Fire Protection Engineering, SFPE, Boston, MA and NFPA, Quincy, MA, 1988 .

"Comparisons of NBS/Harvard VI Simulations and Full-Scale, Multi-room Fire Test Data," J.A. Rockett, M. Morita, and L.Y. Cooper, NBSIR 87-3567, July 1987; presented at 2nd International Conference on Fire Safety Science, June 1988, and to be published in the Conference Proceedings.

"Fire in a Room with a Hole: A Prototype Application of the Consolidated Compartment Fire Model (CCFM) Computer Code," Cooper, L.Y. and Forney, G.P., presented at the Combined 1987 Fall Technical Meeting of the Eastern Section of the Combustion Institute and Annual CFR Research Conference, Nov. 2-5, 1987, and published in the Proceedings.

"Thermal Response of Unconfined Ceilings Above Growing Fires and the Importance of Convective Heat Transfer," L.Y. Cooper and D.W. Stroup, Journal of Heat Transfer, Vol.109, pp.172-178, Feb. 1987.

"Transient Ceiling Jet Characteristics," V. Motevalli, C.H. Marks, B. McCaffrey and L.Y. Cooper, presented at the Combined 1987 Fall Technical 
Meeting of the Eastern Section of the Combustion Institute and Annual CFR Research Conference, Nov. 2-5, 1987, and published in the Proceedings.

"Ceiling Jet-Driven Wall Flows in Compartment Fires," L.Y. Cooper, NBSIR 87 3535, April 1987; to appear in Combustion Science and Technology.

"Calculating Flows Through Vertical Vents in Zone Fire Models Under Conditions of Arbitrary Cross-Vent Pressure Difference," Cooper, L.Y., NBSIR 88-3732, May 1988; also "A Note on Calculating Flows Through Vertical Vents in Zone Fire Models Under Conditions of Arbitrary Cross-Vent Pressure Difference," Cooper, L.Y., submitted for outside publication.

"An Experimental Study of the Transient Thermal Response of Unconfined Ceilings Above Fire Plumes," A. Woodhouse, C.H. Marks and L.Y. Cooper, presented at the 2nd ASME-JSME Thermal Engineering Joint Conference, March 1987, and published in the Proceedings.

"Heat Transfer in Compartment Fires Near Regions of Ceiling Jet - Wall Impingement," L.Y. Cooper, presented at the 1987 ASME/AIChE National Heat Transfer Conference and published in Proceedings; to appear in the Journal of Heat Transfer.

"Test Results and Predictions for the Response of Near-Ceiling Sprinkler Links in Full-Scale Compartment Fires, L.Y. Cooper and D.W. Stroup, NBSIR 87-3633, Sept. 1987; presented at 2nd International Conference on Fire Safety Science, June 1988, and to be published in the Conference Proceedings.

"Before the Smoke Clears - Heat and Mass Transfer in Fires and Controlled Combustion," Kennedy L.A. and Cooper, L.Y., Mechanical Engineering, Vol. 109/No. 4, pp62-67, April 1987.

"A Catalog of Compartment Fire Model Algorithms and Associated Computer Subroutines," D.W. Stroup, NBSIR 87-3607, August 1987.

Proceedings of Sessions on Heat and Mass Transfer in Compartment Fires, Cooper, L.Y. and Farouk, B., Eds, Vol. 1, pp. 309-440, Proceedings of the 2nd ASME-JSME Thermal Engineering Joint Conference, American Society of Mechanical Engineers, New York, NY, March 23-27, 1987.

"Enclosed Buoyant Convection in a two-Layer Stratified Fluid," Rehm, R.G., Lozier, D.W., Baum, H.R., and Cooper, L.Y., to be presented at the 1988 Technical Meeting of the Eastern Section of the Combustion Institute, Dec. 57, 1988, and published in the Proceedings.

"An Algorithm for Modeling Flow Through a Simple Fan-Resistance Ventilation System and Its Application to Fire Modeling," Cooper, L.Y., Klote, J.H., and Duong, D.Q., to appear as NISTIR.

"Model of a Simple Fan-Resistance Ventilation System and Its Application to Fire Modeling," Klote, J.H. and Cooper, L.Y., to appear as NISTIR.

"Negatively Buoyant Wall Flows Generated in Enclosure Fires, Jaluria, Y, and Cooper, L.Y.," invited paper for publication in Progress in Energy and Combustion Science, under review. 


\section{Related Grants}

Compartment Fire Combustion Dynamics, C.L. Beyler, Fire Science Technologies, and R. J. Roby, Virginia Polytechniic Institute.

Experimental Study of the Environment and Heat Transfer in a Room Fire, Zukoski, E.E., California Institute of Technology.

Negatively-Buoyant and Penetrative Flows Generated in Enclosure Fires, Jaluria, Y., Rutgers - The State University of New Jersey.

Numerical Analysis Support for Compartment Fire Modeling Code Development, W.F. Moss, Clemson University.

Prediction of Fire Dynamics, J deRis - Factory Mutual Research Corporation. See p. 31 for description)

Transient Ceiling Jet Characteristics, Marks, C.H., The University of Maryland. 


\section{Center for Fire Research \\ National Institute of Standards and Technology \\ FY 88}

Institution: Virginia Polytechnic Institute and State University

Grant No: 60NANB8D0829

Grant Title: Compartment Fire Combustion Dynamics

Principal Investigators:
Richard Roby
Department of Mechanical Engineering
Virginia Polytechnic Institute and S.U.
Blacksburg, VA 24061
Tel. (703) 961-5846

\author{
Craig Beyler \\ Fire Science Technologies \\ 3215 Donnybrook Lane \\ Cincinnati, OH 45251 \\ Tel. (513) 522-4688
}

Other Professional Personnel: Michael Skelly, Graduate Assistant

NIST Scientific Officer: Dr. H.E. Mitler

Technical Abstract:

As most fire fatalities are the result of exposure to toxic products of combustion such as carbon monoxide, it is essential that methods be devised to evaluate the toxic hazards posed by specific materials in varying building designs. While toxic products are produced during both smoldering and open combustion modes, the rate of generation of toxic products such as carbon monoxide and hydrogen cyanide is greatest under conditions where the compartment flow dynamics create oxygen deficient combustion conditions.

The investigation will experimentally 1) determine the effect of realistic compartment fire flows on species generation rates and their correlation with equivalence ratio, 2) establish major toxic gas and smoke generation rates for important fuels such as wood and TDI-based polyurethane, and 3) determine the efficiency of external flames in destroying major toxic gases produced in the compartment under oxygen deficient compartment fire combustion.

The apparatus currently being constructed for this investigation includes a $1.2 \mathrm{~m} \mathrm{X} 1.2 \mathrm{~m} \mathrm{X} 1.5 \mathrm{~m}$ compartment with two ventilation paths, a normal window style exhaust vent and a ducted air inlet vent (see Figure 1). Separation of the two flows allows an unambiguous measurement of the air inflow, while leaving the exhaust flow dynamics undisturbed. Combined with a measurement of fuel supply rate, this system will allow an accurate determination of the compartment equivalence ratio. Exhaust from the compartment will be collected by a hood system instrumented to allow the measurement of gas and smoke generation rates. Routine measurements 
will include determination of species concentrations for carbon monoxide, carbon dioxide, oxygen, total unburned hydrocarbons, and smoke. In addition concentrations of other important species, such as hydrogen cyanide and hydrochloric acid, will be determined by either gas chromatography or FTIR analysis.

In addition to exhaust measurements, detailed measurements of species and temperature will be made in the compartment and in the external flame. These measurements will support species generation modeling, modeling of the external flame based on a detailed knowledge of the vent flow rate and composition, and evaluation of the existing model for layer ignition under realistic compartment fire conditions. The apparatus can be modified to study flames from external windows or flames from vent openings to internal corridors.

Work on this project was initiated in the final two months of FY 1988 and has focused on construction of the experimental apparatus, modification of the experimental facility, and development of species measurement capabilities.

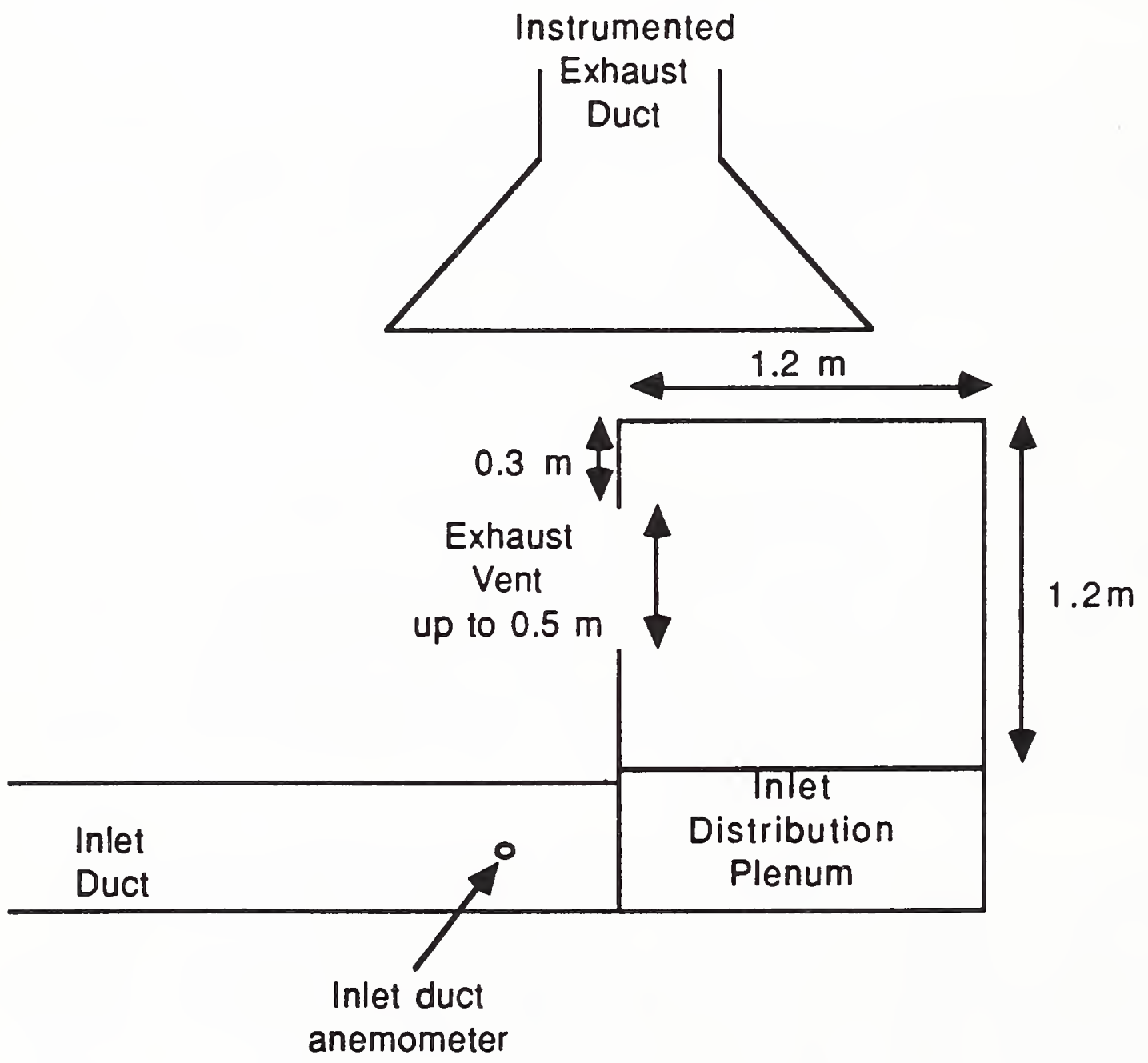

Figure 1. Compartment Layout. 


\title{
CENTER FOR FIRE RESEARCH \\ NATIONAL BUREAU OF STANDARDS \\ FY 88
}

\author{
INSTITUTION: California Institute of Technology \\ GRANT NO.:" NBS5 60NANB600638 \\ GRANT TITLE: Experimental Study of Environment and Heat \\ Transfer in a Room Fire
}

Principal Investigator:

Other Professional Personnel:

NBS Scientific Officer:

\author{
Professor Edward E. Zukoski \\ Jet Propulsion Center \\ 301-46 \\ California Institute of Technology \\ Pasadena, CA 91125
}

Professor Toshi Kubota

Richard Chan, PhD Candidate

James Morehart, PhD Candidate

Dr. Walter Jones

Technical Abstract:

Introduction. Smoke which is produced by a fire spreading through a building can act to aid in the spread of the flame by the transport of hot and perhaps combustible gas, and can be harmful to occupants because of its temperature and toxicity or because its opacity hinders the rapid movement of the occupants through the building. Under certain circumstances, the combustion of the fuel produced in the fire can occur under conditions in which the concentration of oxygen in the ambient gas is far below normal and a fuel $r$ ich mixture can be produced which will burn later in an adjacent space and aid in the spread of the fire. We are interested in developing models for the production and movement of smoke in complex structures and have been studying several fluid dynamic processes which will be described below. These processes are difficult to describe analytically because the flows are turbulent and are strongly influenced by turbulent mixing between streams in which buoyancy forces are predominant and because the chemical processes in turbulent-buoyant flames are not understood completely.

Gravity Currents. The flow of hot gas in a gravity current in hallways or across very wide rooms allows for very rapid transport of gas which may be toxic and which may also be combustible. Our aim is to understand this transport process and, in particular, the influence of heat transfer between the current and the ceiling on the current and on the subsequent development of the ceiling layer in the hallway or room. Gravity currents in hallways which are horizontal and inclined up to 30 degrees below the horizontal are being investigated.

Two types of apparatus are being used: the first is a saltwater/ water facility which is a 2.4 meter long channel with a $15 \mathrm{~cm}$ square cross section. This is being used to investigate the dependence of the gravity current parameters on the Reynolds number and the inclination angle, and to determine the nature of the return flow which develops after the gravity current has impinged on a vertical wall 
closing the downstream end of the channel.

Flow visualization techniques are used to measure the velocity of the head of the current, the depth of the current, and the nature of the return flow. Fluorescent dye techniques are being developed to measure the concentration profiles in the flow and hydrogen bubble techniques, and to determine the velocity profiles.

The work in this facility has now been completed and a description of results is included in the Final Report for this grant year.

The second apparatus is an air facility $8.6 \mathrm{~m}$ long and $0.5 \mathrm{~m}$ square. This apparatus and the experimental results obtained in it are described in detail in the papers by Chobotov et al. Experiments in this apparatus have shown that the rate of heat transfer between the current and the ceiling is much higher than rates calculated for forced convection over flat plates because of a buoyancy controlled instability in the wall boundary layer. The rates are high enough to reduce the overheat in the hot gas to very small values within a length corresponding to 50 times the current depth. The effect on the velocity of the head is smaller but the influence of the heat transfer could lead to a strongly stratified hot gas layer in long hallways.

This apparatus has been used to investigate heat transfer effects on currents with an initial depth of 10 to $15 \%$ of the channel deep. Earthquake damage to this duct is now being repaired and it will be used to study currents which initially occupy between 20 to $30 \%$ of the depth of the channel and the reverse flow produced when the current impinges on the closed end of the hall.

Motion of Smoke in Vertical Shafts. We are also beginning to study the influence of heat transfer on the motion of hot gas in vertical shafts initially filled with cooler air. We plan to use experimental approaches similar to the two discussed here for horizontal flows in these new experiments.

Combustion in Two-Layer Mode1s: We are also studying the products of combustion and flame geometries produced by large buoyancycontrolled diffusion flames which extend from a lower layer, which contains unvitiated air, into an upper layer which contains products of combustion mixed with air. The results of these tests are described in detail in Toner et al (1986) for steady state flows from the plume into the upper vitiated layer.

Toner's measurements were made for steady flows in which the equivalence ratio for the gas entering the upper layer in the fire plume $\phi_{p}$ is equal to that for the gases which make up the upper layer $\phi_{\ell}{ }^{\circ}$

However, in most fires these equivalence ratios are not equal and in general the values of $\phi_{l}$ will be less than the corresponding values for $\phi_{p}$ due the transient development of the fire plume and the upper layer. An important question here concerns the possibility that excess fuel or oxygen in the fire-plume will react with excess oxygen or fuel entrained from the ceiling layer into the fire plume. 
In order to study this situation we have constructed a new apparatus which consists of a hood, $1.83 \mathrm{~m}$ square and $1.22 \mathrm{~m}$ deep with windows on one side which allow photographic and visual observations of the flame within the hood. To allow the modeling of unsteady flows, this apparatus was built so that additional gas can be added to the hood through an axisymmetric array of pipes located in the upper $20 \mathrm{~cm}$ of the hood. The gas is injected through a large number of $1 / 16$ th inch holes to insure rapid mixing between the injected gas and the recirculating flow produced within the hood by the fire plume. The new hood is large enough to allow burners up to at least $0.5 \mathrm{~m}$ in diameter to be used in contrast to the $20 \mathrm{~cm}$ burners used in the steady state measure ments made by Toner in the old $1.2 \mathrm{~m}$ square hood.

Because the added gas is injected outside of the region of flaming combustion, we believe that it can be used to model the unsteady situation since the equivalence ratio for the gas recirculated into the fire plume by the entrainment process will have a different value than that in the plume at the interface.

A typical result of preliminary measurements are shown in the accompanying figure where data for mole fractions for oxygen and carbon monoxide measured for the ceiling layer gas are plotted as a function of the layer equivalence ratio, $\phi_{l}$, and for a wide range of values of $\phi_{p}$. $0 p \in \mathbb{n}$ points were obtained without air injection and closed points with air injection; the crosses are earlier steady state data obtained by Toner.

Values of $\phi_{p}$ were fixed by setting the distance between the burner and the interface, and the fuel flow rate; values of $\phi_{\ell}$ were reduced from the corresponding steady state values by adding air to the upper layer. We have not yet investigated the effect of adding fuel to the upper layer.

Several interesting features are shown here: first, the measured values of the mole fractions for oxygen and carbon monoxide obtained for a number of fixed values for $\Phi_{p}$ lie on a single curve regardless of the values for $\phi_{p}$. Thus, the species formed are independent of $\phi_{p}$ and depend only on $\phi_{\ell}$.

This is a very welcome conclusion because it greatly simplifies the modeling of this process and it may result from the fact that the entrainment of upper layer gas into the fire plume is much larger than the flow at the interface supplied by the fuel flow rate and entrained air.

Second, the mole fraction for oxygen does not go to zero for values of $\phi_{\ell}$ greater than one but remains a few percent above zero even when $\phi_{l}$ is greater than 2.0. This is in contrast to the data obtained in the smaller hood by Toner, shown in these figures as the crosses. We believe that this difference is due to the presence of surface reactions on ceramic fiber used as insulation in Toner's experiments and the absence of these reactions in the present hood which has bare metal walls. 
These experiments were carried out with a $20 \mathrm{~cm}$ burner and with natural gas as the fuel. We plan to extend the work to include $50 \mathrm{~cm}$ burners and several other fuel types including propane and an alcohol.

\section{Reports and Papers}

Zukoski, E., Toner, S., Morehart, J., Rubota, T. (1988), "Species Production in Two-Layer Fire Plumes," Fire Safety Sciences, Proceedings of the Second International Suymposium.
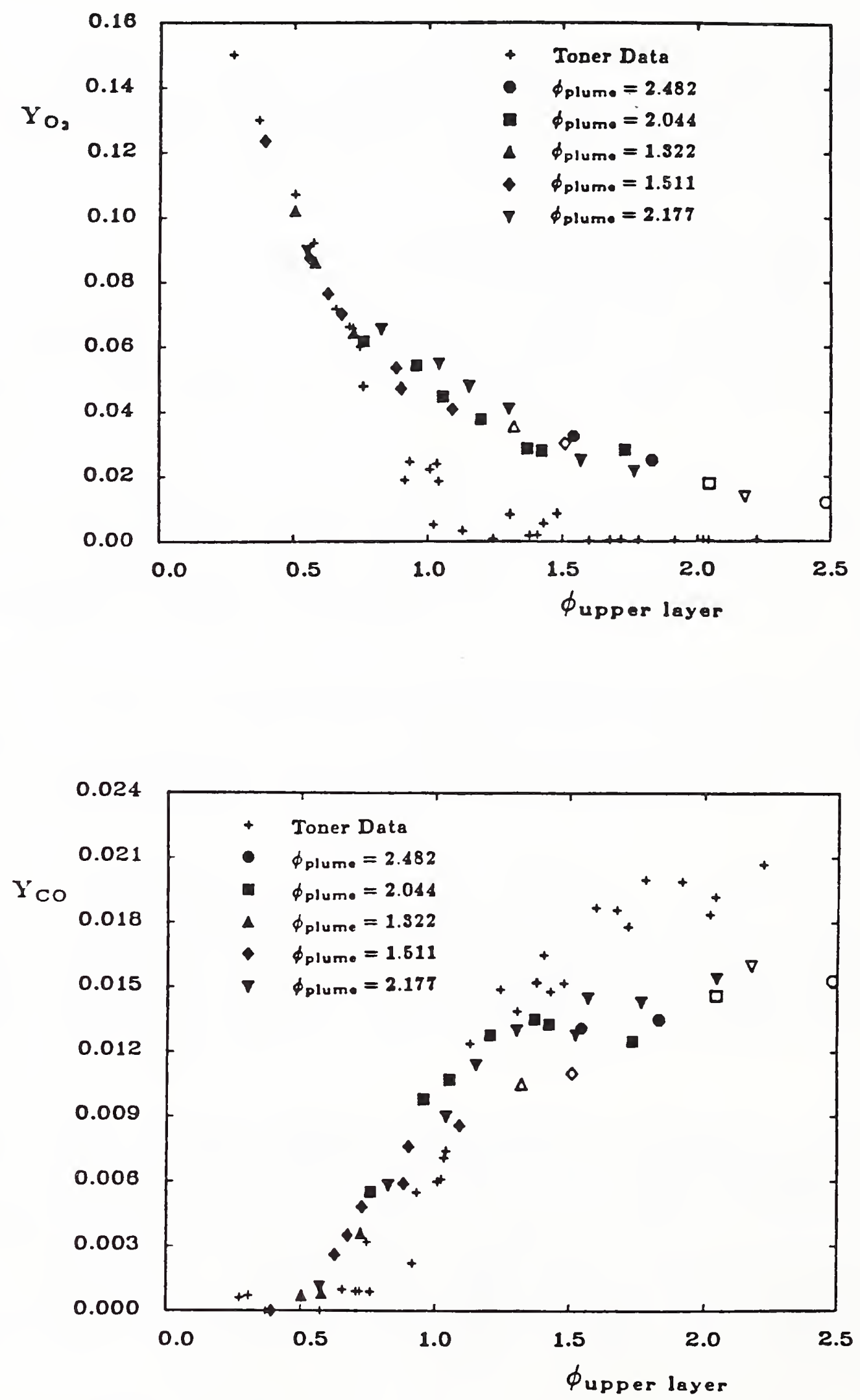


\section{CENTER FOR FIRE RESEARCH \\ NATIONAL BUREAU OF STANDARDS \\ FY88}

Institution:

Grant No:

Grant Title:

Principal Investigator:

Other Professional Personnel:

NBS Scientific Officer:
Rutgers, The State University of New Jersey

New Brunswick, New Jersey

\section{NANB7D0743}

Negatively Buoyant Flows Generated in Enclosure Fires by the Downward Turning of Ceiling Jets

Professor Yogesh Jaluria

Department of Mechanical \& Aerospace Engineering

Rutgers, The State University of New Jersey

New Brunswick, New Jersey 08903

Telephone: (201) 932-3652

K. Kapoor (Post-Doctoral Fellow)

A. Abib (Ph.D. Student)

Dr. Leonard Y. Cooper

Technical Abstract:

Introduction. The present research program extends and continues the earlier work on negatively buoyant and penetrative flows that arise in enclosure fires, with emphasis on the downward turning of the ceiling jet at the corners. Employing and modifying the experimental systems and facilities developed earlier, the research effort considers the flow of a heated two-dimensional jet over a horizontal, isothermal or adiabatic, surface, simulating the ceiling of a compartment, and the downward turning at the corners to give rise to a negatively buoyant wall flow. Of particular interest are the penetration distance, entrainment in to the flow and wall heat transfer in this flow. These are to be correlated with the results obtained earlier for a negatively buoyant wall jet in an isothermal medium. Extensive data have been taken on the flow under consideration. Correlating equations have also been derived for the heat transfer, flow rate and penetration depth as functions of the inlet conditions for the heated jet. The observed trends are similar to those obtained earlier for a negatively buoyant wall jet and quantitative relationships between the two flows are being derived to provide inputs in the modeling of the transport at the early stages of the fire.

Negatively Buovant and Penetrative Wall Flows. A considerable amount of work has been done on negatively buoyant wall jets in extensive media, stratification of an enclosed region in to two essentially isothermal zones, penetration of a wall jet across the interface between the two zones and generation and penetration of a buoyancy induced flow across the interface. Correlating equations have been derived for the penetration distance $\delta$, heat transfer Nusselt number $\mathrm{Nu}$ for an isothermal wall at temperature $\mathrm{T}_{S}$ and the total entrainment into the flow. These equations have been obtained for a wall jet in an extensive medium at temperature $T_{\infty}$ as well as for a jet in a two-layer, stably stratified, environment at upper layer temperature $T_{u}^{\infty}$ and lower layer temperature $T_{\ell}=T_{\infty}$. Figure 1 shows the penetration distance as a function of the Richardson number $\mathrm{Ri}$, based on the inlet slot width $D$, temperature $T_{0}$ and velocity $U_{o}$ for a jet in a two layer environment. Similarly, Figure 2 
shows the corresponding heat transfer in terms of the local Nusselt Number. Several other results were obtained and have been reported in the papers and reports listed at the end of this summary.

Downward Turning of Ceiling Jets. This problem has been under detailed investigation over the current year. Figures 3 and 4 show the resulting isotherms and the surface heat transfer. It is seen that the downward flow is very similar to a negatively buoyant wall jet in an extensive isothermal medium. The basic flow is sketched in Figure 5 and interest lies in correlating the inlet conditions at (c) with those at (1) and (2), so that the ceiling jet flow may be coupled with the negatively buoyant wall jet that arises. The present effort has also been directed at surface heat transfer rates and the entrainment into the flow. It is found that the entrainment is a strong function of the conditions at position (2). The ceiling jet loses thermal energy and momentum as it flows from (c) to (2). Thus, the penetration distance $\delta_{\mathrm{p}}$, heat transfer and the flow rate $\dot{\mathrm{m}}_{\text {out }}$ are determined by the transport occurring at the horizontal surface and in the downward turning. This is the main concern in the present effort and the extensive data taken so far are being analyzed to obtain a quantitative description of the flow and transport processes as the fire-plume-driven ceiling jet spreads over the ceiling and turns downward to generate a negatively buoyant wall flow.

\section{$\underline{\text { Reports and Papers }}$}

1. Jaluria, Y, "Buoyancy-Driven Wall Flows in Enclosure Fires," Proc. 21st Symp. (Int.) Combust, Combust. Inst., Pittsburgh, PA, 151-157, 1986.

2. Jaluria, Y. and Kapoor, K, "Experimental Simulation of Penetrative Flows in a Compartment Fire," Proc. Fall Tech. Meeting. Eastern Sect. Combust. Inst. Gaithersburg, MD, Paper No. 89, 1987.

3. Jaluria, Y. and Kapoor, K. "Importance of Wall Flows at the Early Stages of Fire Growth in the Mathematical Modeling of Enclosure Fires," Combust. Sci. Tech. 59, 355-369, 1988.

4. Kapoor, K. and Jaluria, Y. "Heat Transfer from a Negatively Buoyant Wall Jet," NBS Report NBS-GCR-88-541, 1988, also, Int. J. Heat Mass Transfer, 1988, to appear.

5. Abib, A.H. and Jaluria, Y. "Numerical Simulation of the Buoyancy-Induced Flow in a Partially Open Enclosure," Num. Heat Transfer, 1988, to appear.

6. Kapoor, K. and Jaluria, Y., "An Experimental Study of the Generation and Characteristics of a Two-Layer Thermally Stable Environment," Int. Comm. Heat Mass Transfer, 1988, to appear.

7. Jaluria, Y, "Natural Convection Wall Flows," SFPE Handbook, Sec. 1, Ch. 7, 1988. 


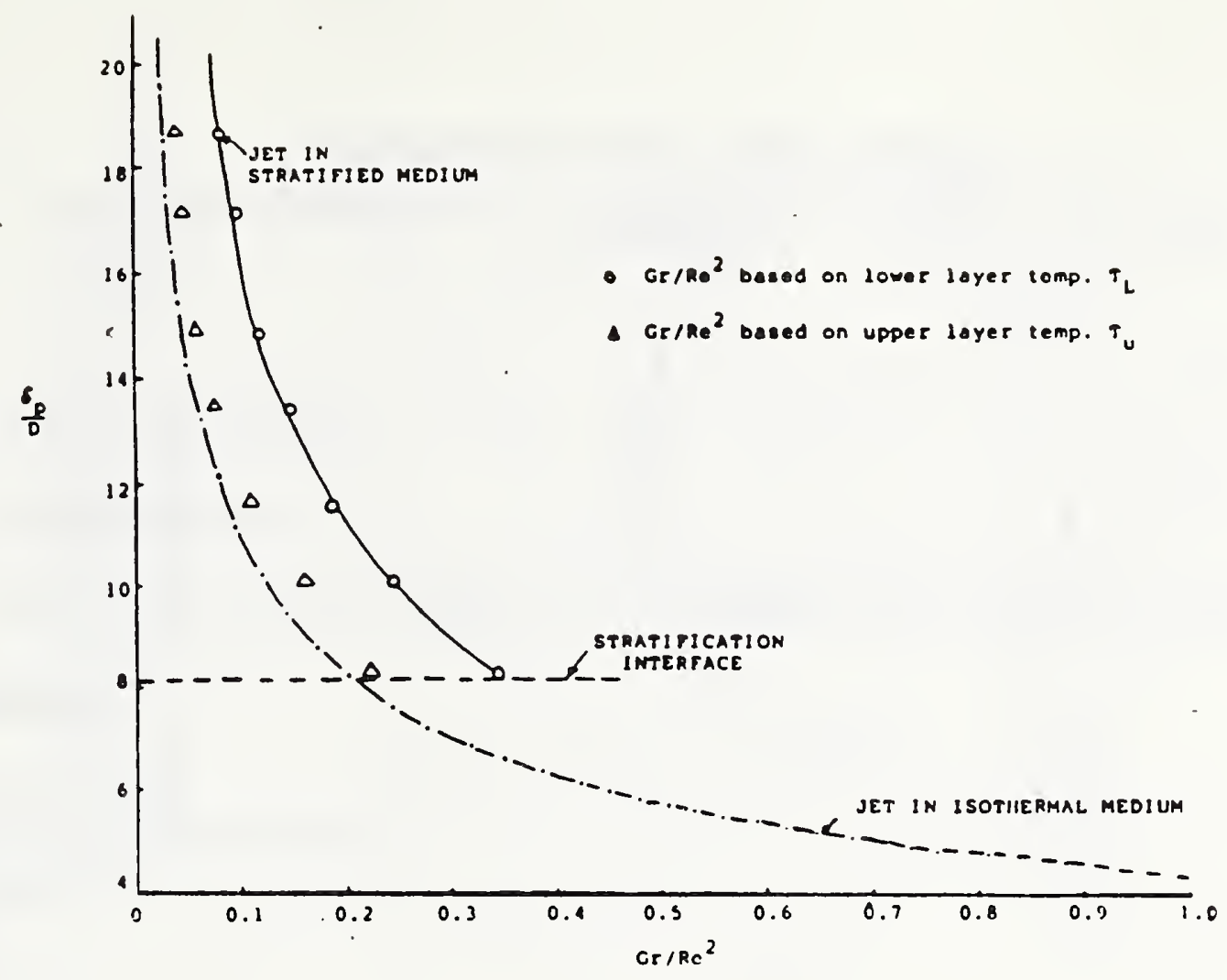

Figure 1. Penetration depth of a negatively buoyant jet in an isothermal and a stably stratified two-layer environment.

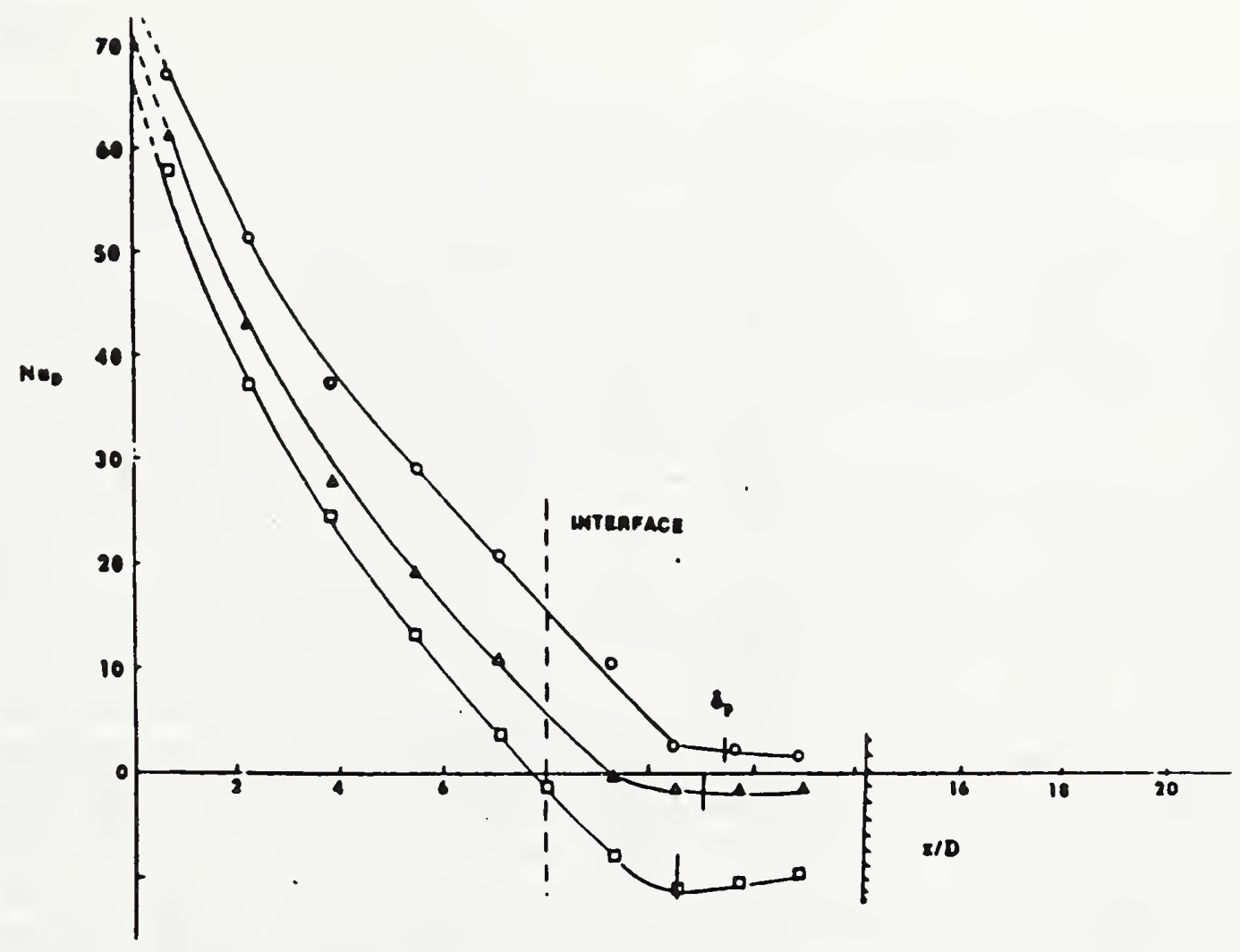

Figure 2. Local heat transfer rate to an isothermal wall due to a wall jet in a two layer environment at $\mathrm{Ri}=0.148 . \quad 0, \mathrm{~T}_{\mathrm{S}} \mathrm{T}_{\infty}=$ $-0.5^{\circ} \mathrm{C} ; \Delta, 12^{\circ} \mathrm{C} ; \square 24^{\circ} \mathrm{C}$. 


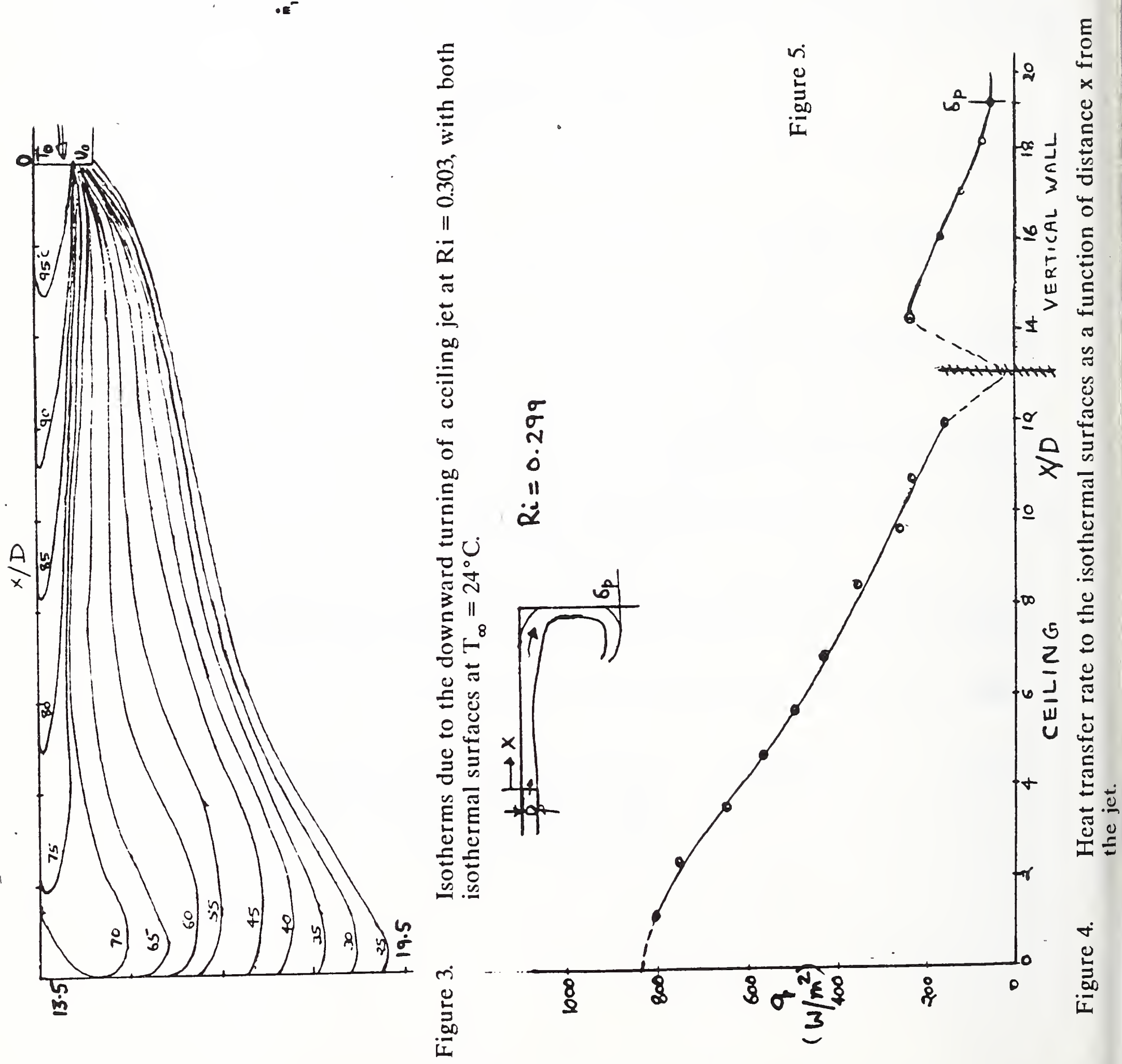




\section{Center for Fire Research \\ National Institute of Standards and Technology \\ FY 88}

Institution: Clemson University

Grant No.: $\quad 60 N A N B 8 D 0857$

Grant Title: Numerical Analysis Support for Compartment Fire Modeling Code Development

Principal Investigators:

William F. Moss

Department of Mathematical Sciences

Clemson University

Clemson, SC

Tel. (803) 656-5225

Other Professional Personnel: Karen Dunlap, Research Assistant

NIST Scientific Officer: $\quad$ Dr. G.P. Forney

Technical Abstract:

The computer code CCFM.VENTS, currently under development by the Building Fire Modeling group, is based on past advancements in compartment fire modeling. This computer code simulates the conditions which develop during the course of an enclosure fire. One or more rooms or compartments are connected together or to the outside by vents such as doors, windows, and holes. Execution of the model requires the solution of a system of ordinary differential equations which solve for pressure, densities, smoke layer height and combustion product concentrations in each room. This investigation will carry out research in support of the numerical and computer science aspects of CCFM code development.

It has already been established that for some problems the system of ordinary differential equations solved by CCFM.VENTS is stiff; that is, if the system is linearized in a neighborhood of a point in time, then the solution of this linear system has terms which are changing rapidly with time and terms which are changing slowly. For such systems, it is essential to use an implicit solver. This means at each step in time a nonlinear system of equations must be solved. Most ordinary differential equation solvers handle this nonlinear problem by using a quasi-Newton method which requires the estimation of the Jacobian matrix associated with the right hand side of the system of differential equations. Most of the work of implicit solvers is involved in approximating the Jacobian matrix and solving a linear system of algebraic equations with this matrix as the coefficient matrix. Methods for exploiting the structure of the Jacobian will be investigated in order to improve the efficiency of CCFM.VENTS. This will allow more complex fire scenarios to be simulated than otherwise would have been possible.

The ODE's in CCFM.VENTS do not always fit the classical definition of stiffness, however. For many problems the Jacobian has eigenvalues with positive real parts. Alternate solution techniques will be investigated to exploit this property of the ODE's.

As illustrated by the peaks in Figure 1 the computer code, CCFM.VENTS, uses a disproportionate amount of computational resources during certain simulation events such as layers crossing vent boundaries. These peaks can also be caused by the use of tabular data such as fire size data that is represented using piece-wise linear interpolation. Both of these phenomena introduce discontinuities into the model equations. Techniques for reducing the impact of these 
discontinuities on the solver will be investigated.

Another source of solution inefficiency is the presence of round-off error or numerical noise that is magnified by sub-model algorithms (plumes, vents, etc.). An error analysis of CCFM.VENT sub-model algorithms will be performed to analyze their error propagation characteristics. For example, if the inputs to an algorithm for computing vent flows have five digits of precision how many digits can one expect from the outputs of the algorithm? This type of cancellation error present in some of CCFM.VENT sub-model algorithms is another source of the peaks found in Figure 1.

Work on this project was initiated in the final month of FY 1988 and has focused on investigating the numerical properties of the CCFM.VENT differential equations.

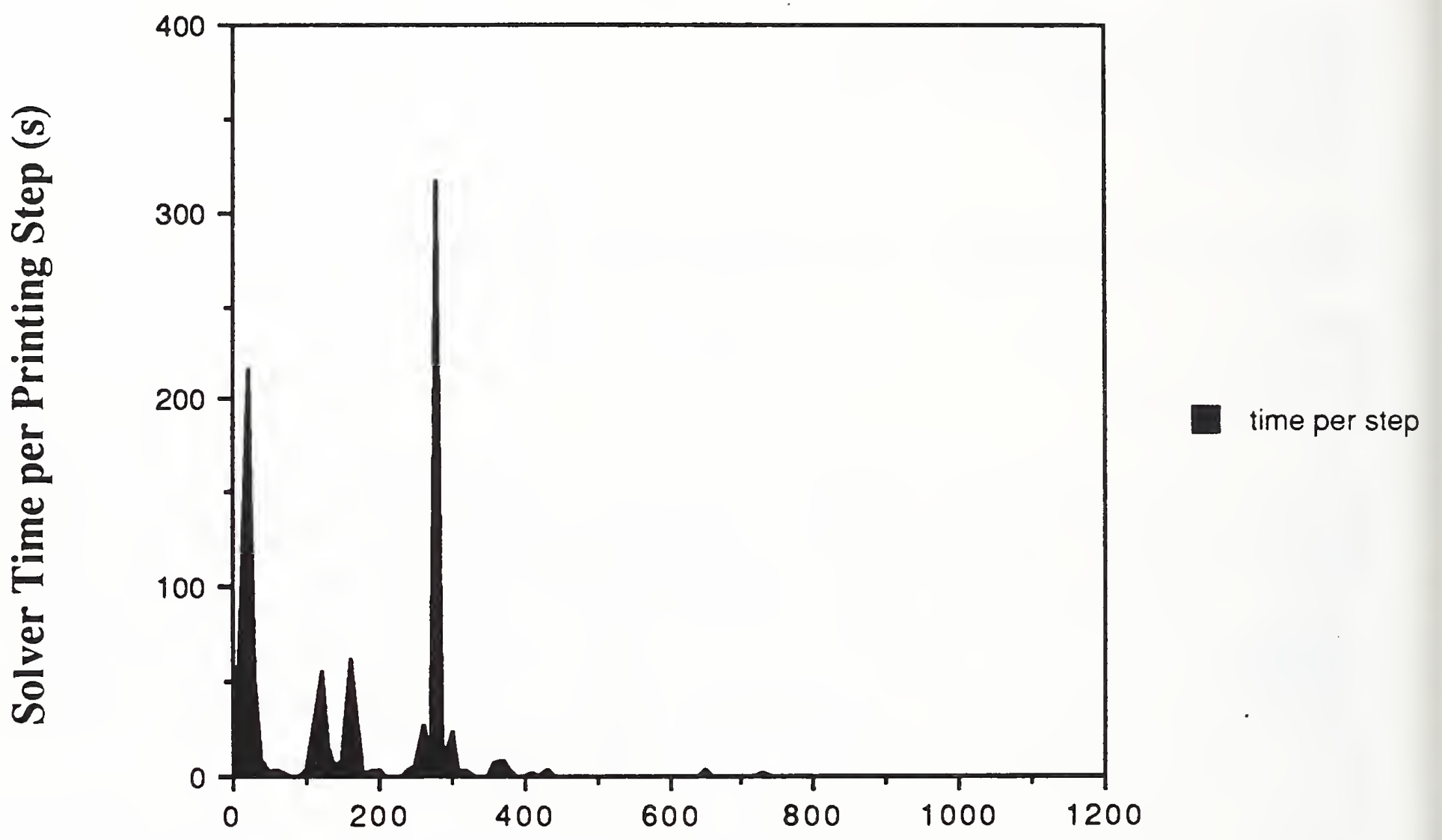

Simulation Time (s)

Figure 1 Plot of Computer time required vs. simulation time 
CENTER FOR FIRE RESEARCH

NATIONAL BUREAU OF STANDARDS

FY 88

Institution: The University of Maryland at College Park

Grant No.: $\quad$ 70NANB 5H0551

Grant Title: Transient Ceiling Jet Characteristics

Principal Investigator: Dr. Colin H. Marks

Mechanical Engineering Department

University of Maryland

College Park, MD 20742

Other Professional Personnel: Vahid Moteval1i, Graduate Student and Guest Worker

NBS Scientific Officer: Dr. David Evans

Technical Abstract:

Introduction This research has been concerned with the experimental determination of transient velocity and temperature profiles in the axisymmetric jet formed by the gases from a fire plume spreading across a ceiling. The purpose of the research is to obtain and provide data for the verification and improvement of compartment fire models, and to aid in the design of sprinkler and smoke detector systems.

The experiments have been on a small-scale with the heat source consisting of a flame produced by the combustion of a premixed stoichiometric mixture of methane and air. The fire strengths ranged from $0.5 \mathrm{kw}$ to $2 \mathrm{kw}$, and the burnerorifice-to-ceiling heights were $0.5 \mathrm{~m}$ and $1 \mathrm{~m}$; this combination of five strengths and heights produced a range of non-dimensional five strength $Q^{*}\left(Q^{*}=\right.$ $Q / \rho_{a m b} C_{p} T_{a m b g} 1 / 2^{5 / 2}$ ) of from $1.85 \times 10^{-3}$ to $1.047 \times 10^{-2}$. During a given run, the fire strength was kept fixed. The transient effect was caused by the variation of the ceiling temperature as it increased from room temperature to its steadystate value. The ceiling in these experiments consisted of a $2.13 \mathrm{~m}$-diameter, $1.27 \mathrm{~cm}$ thick disk of fiberboard. It was insulated on its upper side with a $8.26 \mathrm{~cm}$-thick layer of fiberglass insulation and painted black on its lower surface with an ultra-flat black paint to yield a stable and known emissivity of 0.9 .

Velocities were determined from the time shift in the temperature-time records obtained from pairs of fine ( $1 \mathrm{mill}$ diameter) thermocouples with one thermocouple in each pair mounted a short distance downstream from the first. Temperatures were obtained directly from the thermocouple readings. A more detailed description of the instrumentation and the data-reduction techniques is given in NBS-GCR-88-540. 
Discussion The data obtained are far too voluminous to be presented herein; hence, only a few salient features of them will be presented. Typical temperature and velocity profiles for unconfined ceilings were shown in last years' summary (NBSIR 87-3650) and will not be repeated here. Velocity profiles at a given position were found to be independent of time, whereas temperature profiles reflected the increase of ceiling temperature with time. Both profiles have a maximum point located some distance below the ceiling. The location and magnitude of these maxima is important in the design of sprinkler and smoke alarm systems. Figure 1 shows the distance from the ceiling of the maximum velocity, $\delta_{\mathrm{vm}}$, as a function of radial distance, $r$, from the plume center. Both of these quantities have been normalized with respect to the burner-orifice-toceiling distance, $H$. The data shown are the average for all five strengths for two different heights and for two different times. Some difficulty exists in obtaining $\delta_{\mathrm{vm}}$ exactly because of the scatter in the velocity data, and because the velocity curves are not sharply peaked; however, within the scatter in the data, it appears that $\delta_{\mathrm{vm}} / \mathrm{H}$ depends on $1 \mathrm{y}$ on $\mathrm{r} / \mathrm{H}$, and is independent of time and of H. Cooper's model (1), which is based on momentum-driven wall jets, agrees we11 with the data even in the region beyond $r / H=l$ where buoyancy effects start to become important.

The location of the temperature maximum in the ceiling jet is a function of many more variables than the location of the velocity maximum since the thermal properties of the ceiling must be considered in addition to the condition in the plume. The normalized distance of the temperature maximum, $\delta \mathrm{Tm}$, from the ceiling is shown in Figure 2 as a function of $\mathrm{r} / \mathrm{H}$ for various times. It is clear that $\delta_{\mathrm{Tm}}$ increases with increasing $\mathrm{r} / \mathrm{H}$ and that it decreases with increasing time. Effort is being expended to construct a model which will correlate the $\delta_{\mathrm{Tm}}$ data and so enable a better prediction of the location of the temperature maximum location.

The maximum steady-state velocity, $V_{\max }$ and maximum steady-state temperature difference, $\Delta \mathrm{T}_{\max }$, between the jet and the ambient air are plotted in Figures 3 and 4. As mentioned earlier, the velocity is independent of time. The data have been normalized in a form used by Byler(2). The normalized ordinate in each case is not non-dimensional since the fluid properties and gravity have been left out to simplify the normalized form. kgs units have been used in these figures. The data in each figure correlate we11, and curve.fitting yields the following equations for $r / H \geqslant 0.26$.

$$
\begin{aligned}
& \mathrm{V}_{\max } / \mathrm{Q}^{1 / 3} \mathrm{H}^{-1 / 3}=0.017(\mathrm{r} / \mathrm{H})^{-2}+0.113(\mathrm{r} / \mathrm{H})^{-1}+0.097 \\
& \Delta \mathrm{T}_{\max } / \mathrm{Q}^{2 / 3} \mathrm{H}^{-5 / 3}=0.12(\mathrm{r} / \mathrm{H})^{-2}+5.43(\mathrm{r} / \mathrm{H})^{-1}+2.48
\end{aligned}
$$

The normalized velocity and normalized temperature decrease rapidly with increasing $\mathrm{r} / \mathrm{H}$ at small values, but become almost constant at large $\mathrm{r} / \mathrm{H}$.

Also shown in these figures are correlations due to Alpert(3), Heskestad and Delichatsios(4) and Cooper(1). Our maximum velocity data seem to be in better agreement with Alpert's results than the correlations of Cooper or those of 
Heskestad and Delichatsios, and seem to generally fall between the curves. The maximum temperature data have the same general shape as the curves of the other investigators but have higher values. Differences are to be expected due to different ceiling materials and different conditions on the ceilings upper surface.

Normalized steady-state velocity and Temperature Profiles The steady-state ceiling jet velocity and temperature profiles, with $\mathrm{V}$ and $\Delta \mathrm{T}$ normalized in the same way as $V_{\max }$ and $\Delta \mathrm{T}_{\max }$ according to Eqs. $(3 \& 4)$, are shown for the $\mathrm{r} / \mathrm{H}$ location of 0.26 in Figures 5, and 6 . The distance away from the ceiling, $y$, is normalized by the floor-to-ceiling height. The velocity profiles appear to collapse very well onto a single curve. There is some scatter in the data which is generally due to changes in the room and variations in the experimental conditions. Some of the scatter may be due to the resolution in selecting the time shift. The normalized temperature profiles in Fig. 6 show relatively good agreement between the results for the range of $Q^{\prime}$ 's and $H^{\prime}$ s used.

\section{References}

1. Cooper, L.Y., "Ceiling Jet-Driven Wall flows in Compartment Fires", NBSIR 87-3535, April, 1987.

2. Beyler, G.L., "Fire Plumes and Ceiling Jets", Fire Safety J., 11, 53-75, 1986.

3. Alpert, R.L., "Fire Induced Turbulent Ceiling Jet", Technical Report, Factory Mutual Corp., FMRC Serial No. 19722-2, May 1971.

4. Heskestad, G. and Delichatsios, M.A., Proc. 17th Int'1 Symp. on Combust., Pittsburg, 1113, 1978.

\section{Reports and Publications}

1. Motevalli, V., C. Marks and B. McCaffrey, "Measurement of Velocity and Temperature Profiles in Low-Speed, Turbulent, Non-Isothermal Flows", ASME paper 非7-WA/HT-16, 108th ASME Winter Annual Meeting, Boston, Mass., Dec. 1987. Also published by National Bureau of Standards as report 非BS-GCR$87-535$.

2. Motevalli, V., C. Marks and B. McCaffrey, "Transient Ceiling Jet Characteristics", Eastern Section: Combustion Institute, 20th Fal1 Technical Meeting, Gaithersburg, MD, Nov. 1987, pp. 88-1 to 88-4.

3. Marks, C.H. and V. Motevalli, "Transient Ceiling Jet Characteristics", Summaries of Center for Fire Research In-House Programs and Grants - 1987, NBSIR 87-3650, National Bureau of Standards, Gaithersburg, MD, Oct. 1987, pp. $137-140$.

4. Motevalli, V. and C.H. Marks, "Transient Characteristics of Unconfined FirePlume-Driven Ceiling Jets", Annual Report for National Bureau of Standards grant 非ONANB5H0551. Also published as NBS-GCP-88-540. 


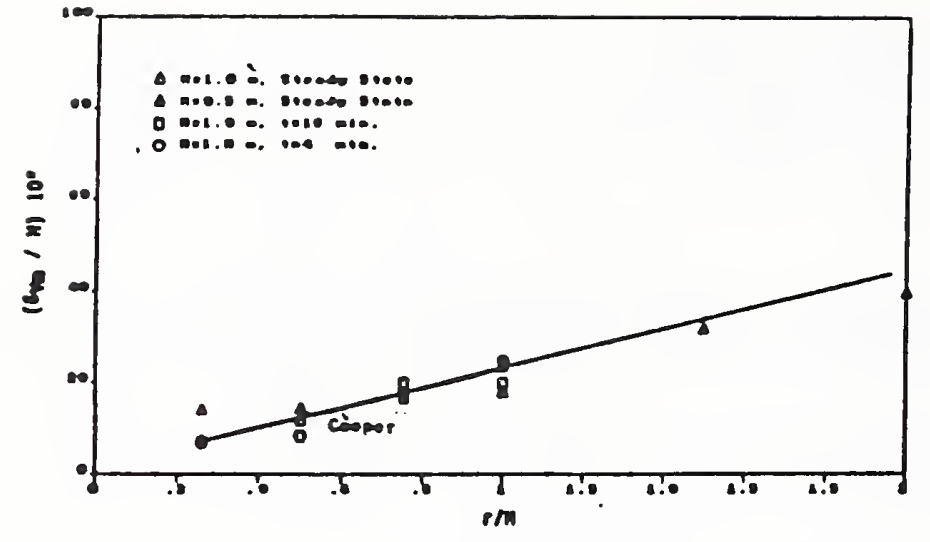

Fig. 1 Position of maximum velocity

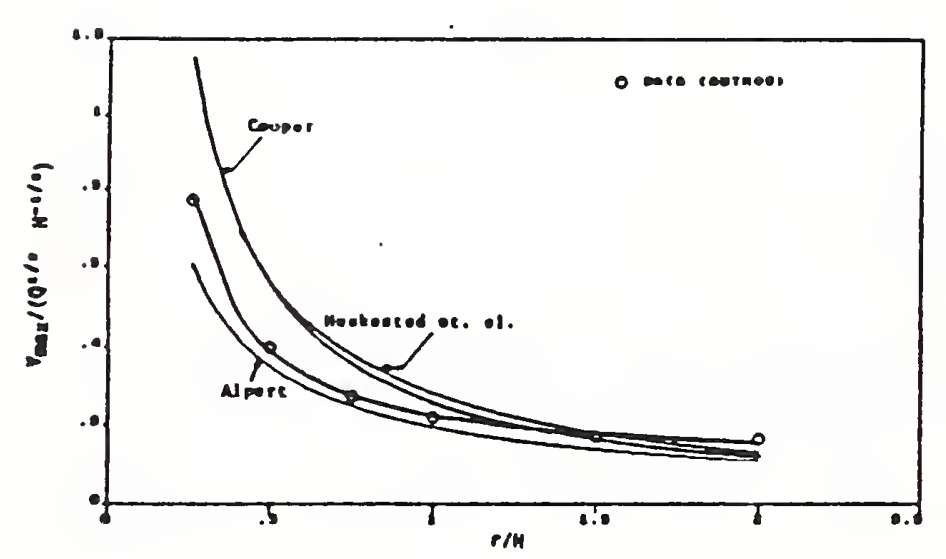

Fig. 3 Maximum velocity as function of radial position

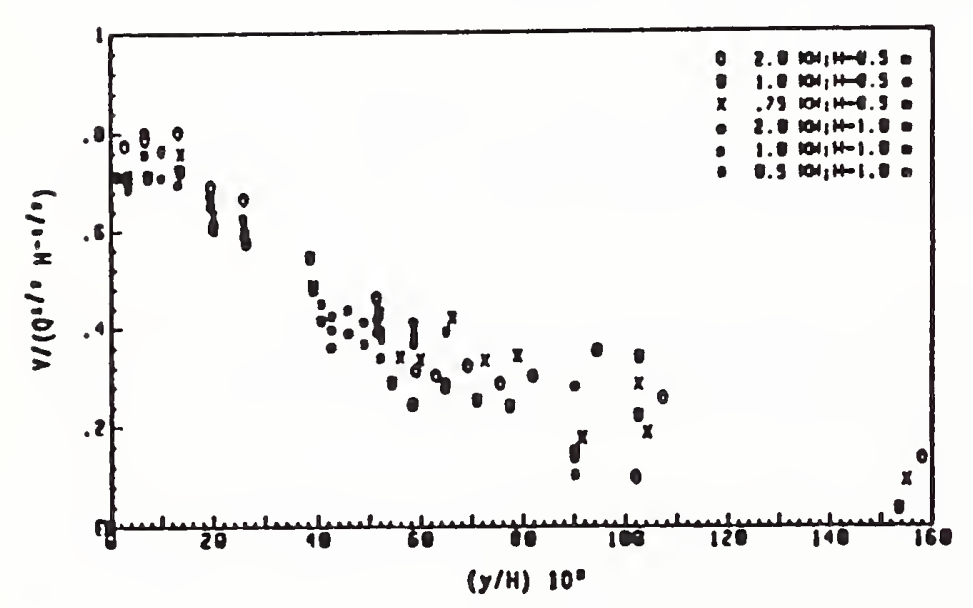

Fig. S Normallzed velocity proflle for $\mathrm{z} / \mathrm{H}=0.26$

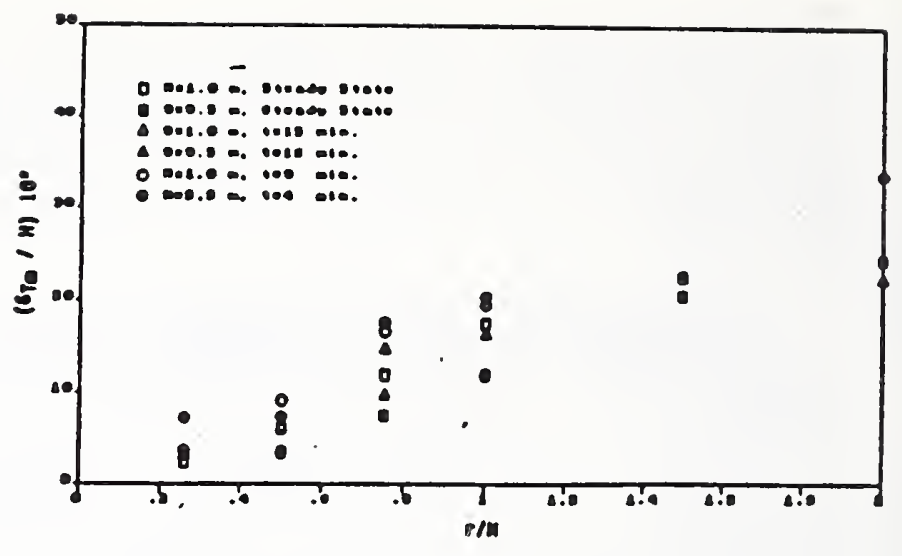

Flg. 2 Position of maxlmum temperature

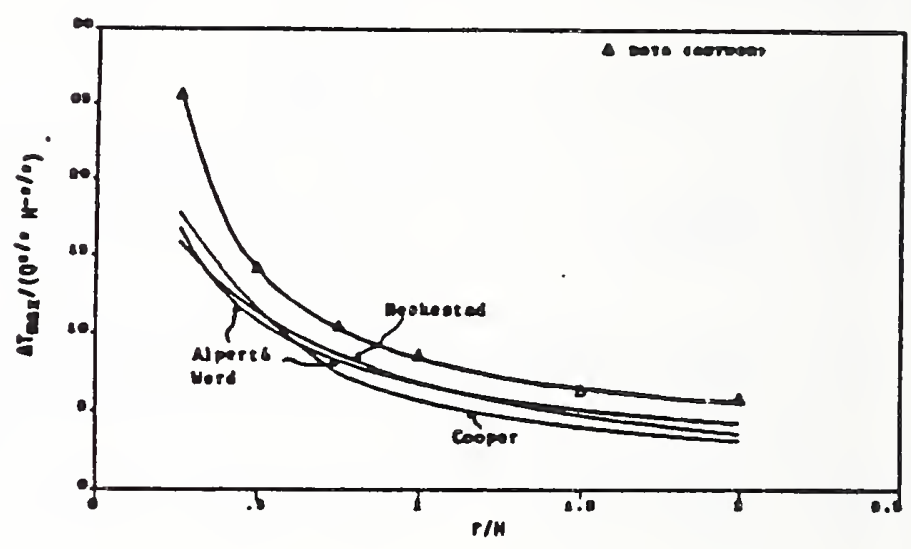

Fig. 4 Maximum temperature difference as a function of radial position (steady state)

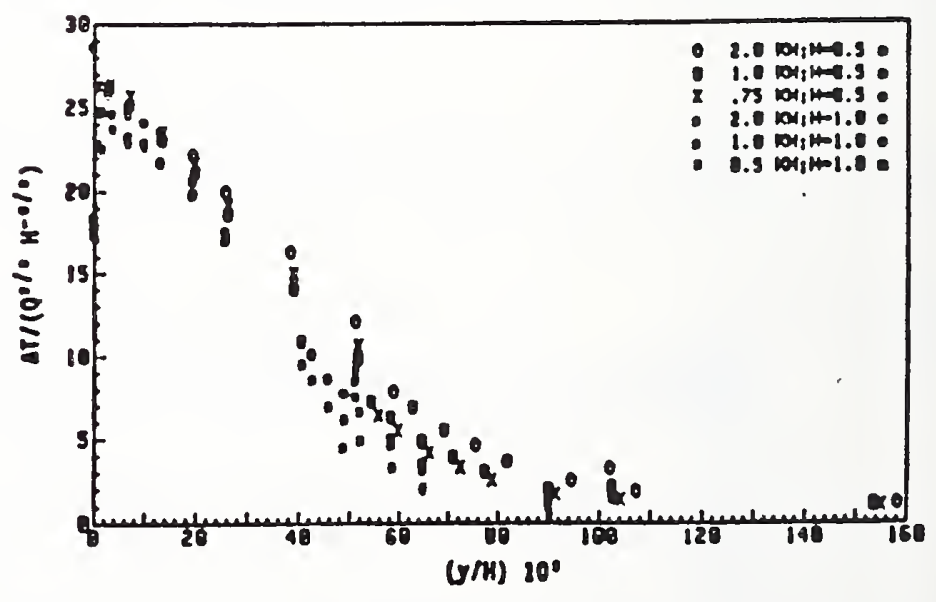

Fig. 6 Normallzed temperature difference proflie for $r / H=.26$ (steady state) 


\section{CEILING FLAME LENGTH}

Professional Personnel

Daniel Gross, Project Leader

\section{Project Objective}

To measure the extension of flames beneath ceilings under buoyancy-controlled conditions.

\section{$\underline{\text { Scope }}$}

The two principal configurations examined were (1) an axisymmetric gas burner flame with and without a horizontal ceiling and (2) a gas burner flame adjacent to a corner-wall and below a confining horizontal ceiling. The natural gas supply range corresponded to energy input rates up to $400 \mathrm{~kW}$ using burners of 0.61 and $0.91 \mathrm{~m}$ diameter in the axisymmetric configuration and a $0.54 \mathrm{~m}$ square burner in the corner-wall configuration. The mean flame tip location was estimated using several techniques: (a) visual (b) $35 \mathrm{~mm}$ time-exposure color photography (c) video photography (d) temperature gradients using thermocouples and (e) oxygen and carbon dioxide concentration gradients.

\section{Technical Accomplishments}

Measurements were made of luminous gas flame lengths with and without ceilings under steady burning conditions and expressed in dimensional and nondimensional terms. The combined vertical and horizontal extension of the intermittent flame tip in the presence of a ceiling was found to be approximately $20 \%$ less than the vertical flame height for the corresponding energy release rate without a ceiling. The accompanying temperature and gas concentration measurements suggest that this was a result of higher temperatures and therefore more complete burning, although no analysis was made of air entrainment or of the overall heat balance.

\section{$\underline{\text { Reports and Publications }}$}

"Measurements of Flame Lengths Under Ceilings", D.Gross, National Institute of Standards and Technology Internal Report NISTIR 88-3835 August 1988 (also submitted to Fire Safety Journal).

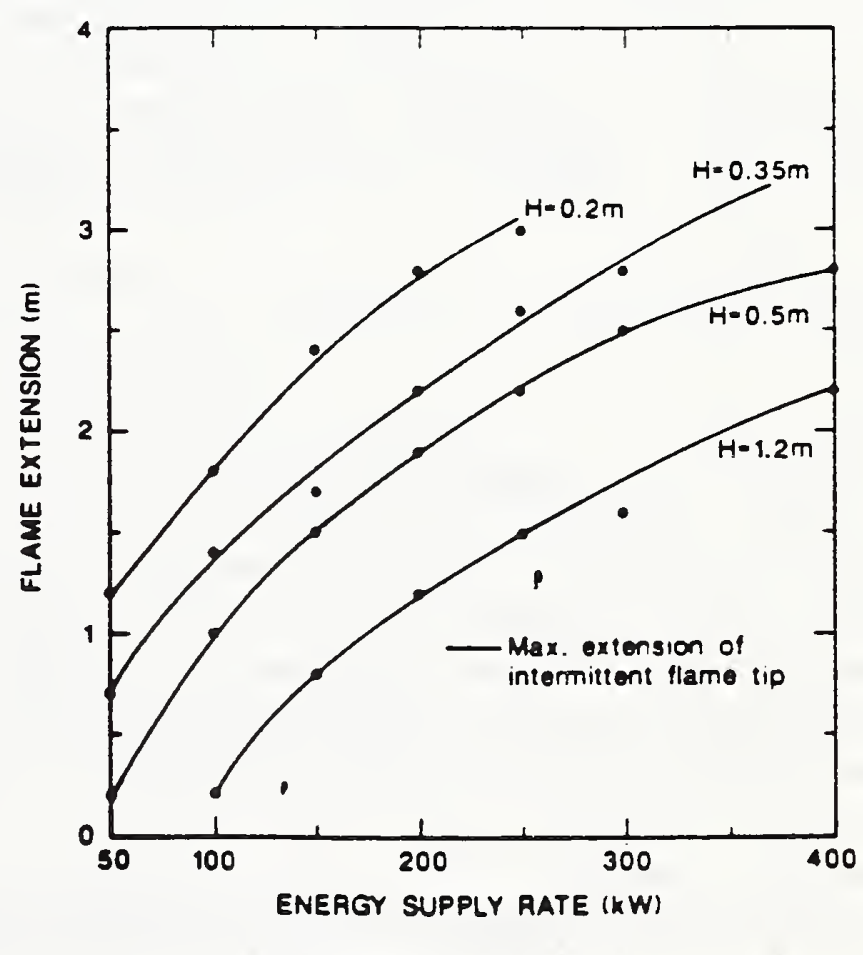

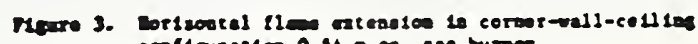

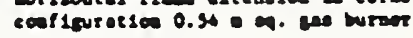




\section{MODEL ACCURACY ASSESSMENT}

\section{Professional Personnel}

Richard D. Peacock, Project Leader

Emil Braun, Physicist

Sanford Davis, Chemist

Walter W. Jones, General Physical Scientist

$J$. Newton Breese, Computer Programmer

C. Lynn Forney, Mathematician

\section{Project Objective}

The objective is to develop the generic methodology to provide a quantitative assessment of the accuracy and limitations of computer based fire models.

\section{$\underline{\text { Scope }}$}

The Center for Fire Research has been developing mathematical models for predicting the conditions which occur as a fire develops and spreads within a room as well as to the rooms connected to it. These models must be validated in order to become useful tools in determining and solving problems related to fire safety. Currently work is being done to establish a methodology for assessing the validity of the mathematical models developed. This assessment problem is likely to require as much time and effort as the model development itself. It includes four broad steps: 1) conceptual model validity - checking that the theory and underlying assumptions are correct and/or reasonable, 2) computerized model verification - ensuring that the computer programming and implementation of the conceptual model are correct, 3) operational validity - determining that the model's output behavior has the accuracy required for the model's intended purpose, and 4) data validity checking that the data used to develop and validate the model are adequate and correct.

\section{Technical Accomplishments}

During the current year, activity focused in two areas: 1) continued development and refinement of the experimental data to be compared with the models, and 2) an analysis of the sensitivity of one of the simpler models which serves as a precursor to the analysis of the more complicated models.

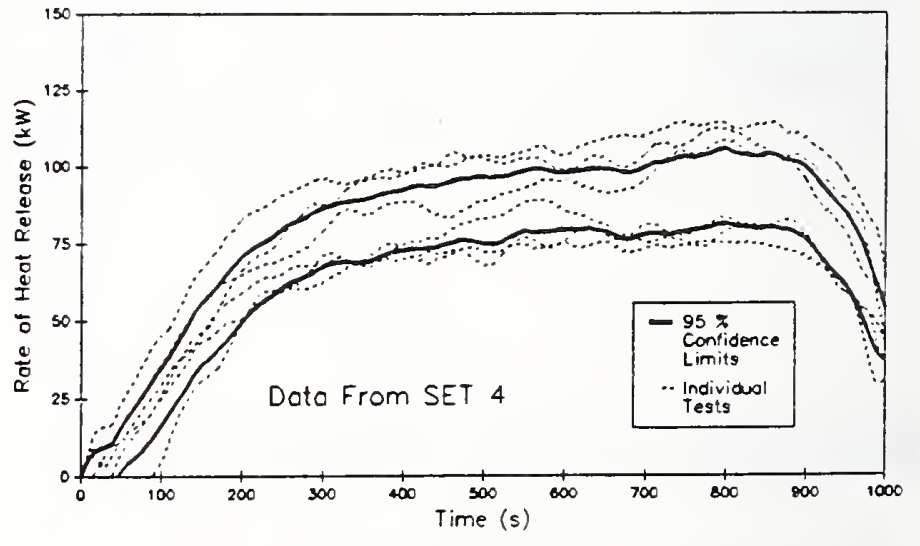

Figure 1

Measured rate of heat release measured by oxygen consumption calorimetry during several tests along with estimated repeatability. 
Several full scale experiments have been performed for testing two-zone fire growth and / or spread models. The experimental arrangement consisted of a burn room and a 12.19-

m long corridor with a target room on one side. A diffusion flame burner using natural gas served as the fire source. In an attempt to develop statistically meaningful data for each test situation, each test combination of fire size and corridor configuration was repeated numerous times. The primary data from the experiments was provided by thermocouples, smoke meters and pressure probes. Details of the methodology used in the experiment are discussed in a
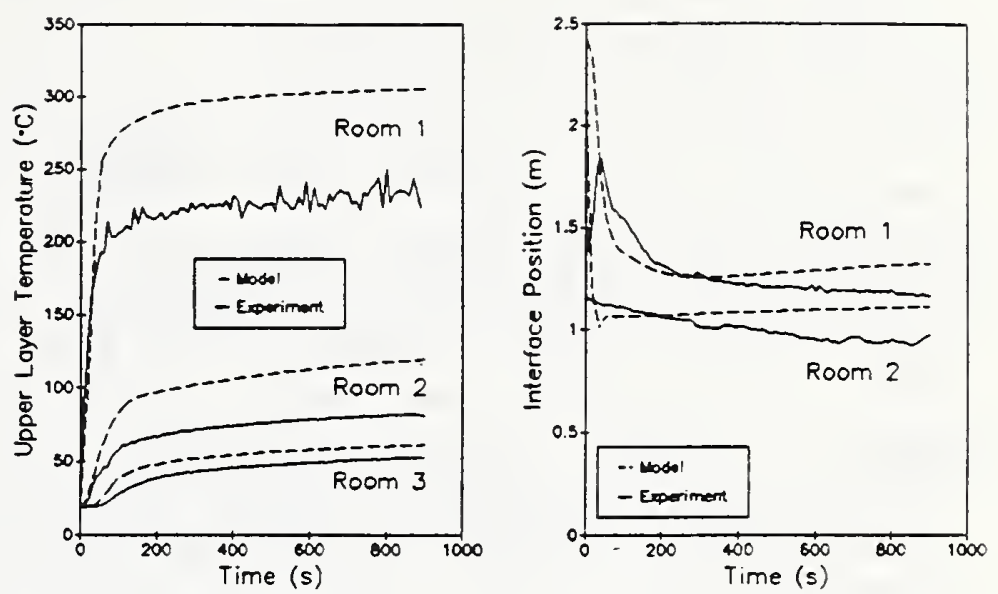

Figure 2

Comparison of measured and predicted upper layer temperature and layer interface position in a three room test facility. report on the experiments. Although it is unreasonable and not particularly useful to present all of the data collected from every test conducted, figure 1 shows some of the data from one data set and illustrates the data from the individual tests together with the average and standard deviation of the set of tests ( 8 in this case) as a function of time. The complete set of data from these experiments provide an independent set of experimental data for comparison with multiple room two-zone fire growth and spread models. As a sample of the use of the data, figure 2 shows the measured upper layer temperature and interface height as a function of time for the three rooms in the test facility and compares it to predictions from one of the current models.

In a sensitivity analysis, the model outputs are examined to see how they respond to perturbations in the input parameters. Since it is often the case that the model inputs are not known precisely, it is important to know how much the outputs can be changed by errors in the input. To investigate methods of determining model sensitivities, the model ASET-B was selected. ASET-B is a fairly simple single compartment, two-zone fire model with five input parameters and two time-dependent output variables. The relatively small size of this model makes the investigation of sensitivity analysis methods tractable. In the future, more complex models will be studied.

ASET-B is fairly insensitive to the uncertainty in the room area and height above the burner, but can be quite sensitive to the uncertainty in the value of $\lambda_{c}$, the fraction of energy from the fire which is transferred by convection. Since the objective of ASET is to be able to determine available safe egress time, $\lambda_{c}$ can be selected according to whether time to detection or time to hazardous conditions is desired. (ASET=time to hazardous conditions-time of detection.) As Cooper ${ }^{1}$ suggests, select $\lambda_{c}$ near .6 to obtain a conservative estimate of the time until hazardous conditions occur and .9 to obtain a conservative estimate of the time until detection (by occupants or detectors) occurs because the fire progresses more quickly as $\lambda_{c}$ becomes smaller. In general, if $\lambda_{c}$ is thought to

1 Cooper, L. Y., A Mathematical Model for Estimating Available Safe Egress Time in Fires, Fire and Materials, Vol. 6, Nos. 3\&4, pp 135-144, September/December 1982. 
be within $x$ of $\lambda$, then choose $\lambda_{c}$ to be $(1-x / 100) \lambda$ for a conservative estimate of the time to hazardous conditions and $(1+\mathrm{x} / 100) \lambda$ for a conservative estimate of the time until detection.

The decoupled direct method selected for the ASET-B analysis seems to provide reasonable error bands on the ASET-B solution given a 108 change in area, $\lambda_{c}$, or height above the burner and also can provide error bands on the time to reach a particular temperature or height. The cost to provide these error bands is that of deriving the sensitivity equations and solving a matrix equation. For ASET-B this effort was small compared to the information provided. For other models, it may be too difficult and complicated to derive the sensitivity differential equations analytically; however, it may be reasonable to compute the Jacobian of $f$ and the partial derivatives of $f$ with respect to the parameters numerically instead. A shortcoming of this method is that it only provides error bands for the case where one input is perturbed. However, a crude approximation for perturbation of more than one variable (for the interface height in ASET-B, $\zeta\left(t, v+v_{1} e_{i}+v_{j} e_{j}\right)$ or for the temperature $\left.\phi\left(t, v+v_{i} e_{i}+v_{j} e_{j}\right)\right)$ can be obtained with $\zeta(t, v)+\left(\partial \zeta / \partial v_{i}\right) v_{i}+\left(\partial \zeta / \partial v_{j}\right) v_{j}\left[\phi(t, v)+\left(\partial \phi / \partial v_{i}\right) v_{i}+\left(\partial \phi / \partial v_{j}\right) v_{j}\right]$. To improve this estimate an additional set of differential equations can be derived from the first order sensitivity equations that would provide the second order sensitivity coefficients. To do this, however, may be impractical as it requires solving $\mathrm{m}^{2} \mathrm{n}$ additional equations where $\mathrm{m}$ is the number of parameters and $\mathrm{n}$ is the number of original equations.

It was seen that the sensitivities of the interface height to area, height above the burner and $\lambda_{c}$ seem to behave (with respect to shape) in the same manner across the parameter space considered. As expected, the interface height is initially sensitive to the height above the burner, but becomes less and less sensitive with time. Initially the interface height appears to be insensitive to $\lambda_{c}$. At time $t=180$ seconds, the fuel source is jumped from $1 \mathrm{kw}$ to $25 \mathrm{kw}$ making the effect of $\lambda_{c}$ more pronounced and the sensitivity of interface height with respect to $\lambda_{c}$ appears to become linear in time. The interface height sensitivity to area rises rapidly to .6-1.5 meters initially. It then levels off with a slight downward trend until the interface height reaches the burner height where it begins to rise. When $\lambda_{c}$ is at a value near .6, the interface height is less sensitive to $\lambda_{c}$ than to area; however, when $\lambda_{c}$ is near .9, the functions of sensitivity with respect to $\lambda_{c}$ and area cross each other with $\lambda_{c}$ becoming the

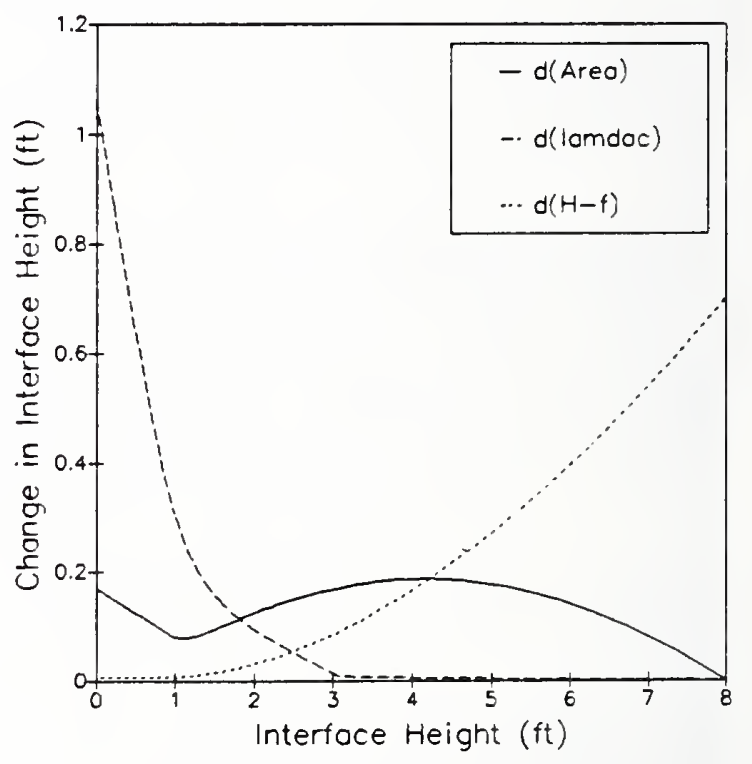

Figure 3.

Effect of a $10 \%$ change in the input parameters of the ASET-B model on the predicted height of the layer interface. dominant parameter. The study indicates that the error in interface height due to a 108 change in area is bounded by approximately $.1 \mathrm{~m}$. The error do to a $10 \%$ uncertainty in height above the burner is bounded by $10 \%$ until the interface height reaches the burner height. From this point on the error is bounded by $.01 \mathrm{~m}$. 


\section{Reports and Publications}

Jones, W. W., and Peacock, R. D., Refinement and Experimental Verification of a Model for Fire Growth and Smoke Transport, paper presented at the Second Annual Symposium on Fire Safety Science, Tokyo, Japan (June 1988).

Peacock, R. D., Davis, S., and Lee, B. T., An Experimental Data Set for the Accuracy Assessment of Room Fire Models, Nat. Bur. Stand., (U. S.), NBSIR 883752 (April 1988).

Peacock, R.D., Status of Predicting Real Fires and Their Effects, States Symposium on Fire Toxicity, Salt Lake City, UT, 29-35 (November 19-20 1987).

Davies, A. D., Some Tools for Fire Model Validation, Fire Technology, Vol. 23, No. 2, May 1987 pp 95-114.

Braun, E., Davis, S., Forney, C. L., and Peacock, R. D., Procedure for the Evaluation of Computer Fire Models, NBSIR in preparation.

Davis, S., and Braun, E., Validation of Fire Models, paper presented to International Symposium on Mathematical Modeling and Related Fire Test Methods, American Society for Testing and Materials, December 1986. To be published in The Journal of Fire Sciences.

Forney, C. L., A Sensitivity Analysis of a Simple Fire Growth Model, to be published

\section{Related Grant}

J. P. Jarvis and M. Kostreva - Clemson University, "Graphical Presentation and Numerical Analysis of Fire Data for Model Validation." 
CENTER FOR FIRE RESEARCH

NATIONAL BUREAU OF STANDARDS

FY 88

Institution: Clemson University

Grant No: $\quad$ 60NANB6D0627

Grant Title: Graphical Presentation and Numerical Analysis

of Fire Data for Model Validation

Principal Investigator: James P. Jarvis. Professor

Department of Mathematical Sciences

Clemson University

$0-104$ Martin Hall

Clemson. SC 29634-1907

Telephone: (803) 656-5214

Other Professional Personnel: Michael M. Kostreva. Associate Professor Cheryl L. Forney, Research Associate

NBS Scientific Officer: Richard Peacock

Technical Abstract:

Introduction. The objective of this work is the development of tools and procedures for validation of computerized fire models. The areas of emphasis have been (1) dimensional analysis and determination of core parameters and (2) sensitivity analysis for physical parameters. The research has been done in large part in the context of specific models developed at the Center for Fire Research (CFR) at the National Bureau of Standards. The ASET model

(Available Safe Egress Time), developed by Cooper [1,2] has been of particular interest.

The validation process involves the comparison of experimental results with model predictions. We are primarily concerned with operational validity determining whether a model's output behavior has the accuracy required for the model's intended purpose. The use of dimensional analysis and determination of core parameters relate to issues of efficiency and simplification of a computer model. Sensitivity analysis deals with the nature of changes in a model's output as a function of changes in its input.

Dimensional Analysis and Core parameters. The main idea in dimensional analysis is to exploit relationships among a model's physical parameters to produce a smaller, but still complete, set of "core" parameters. As a simplistic example, if two parameters appear in a model only as a ratio, those two parameters can be replaced by a single new parameter, the ratio. The advantages of dimensional analysis and reduction to core parameters are in enhanced model simplicity and fundamental understanding of model dynamics. In addition, computational efficiency is increased through avoidance of unnecessary duplication in ranging parameter values when applying the model. It should be noted that the choice of a minimal set of core parameters is not unique and that their choice is not well defined.

These concepts have been applied to the ASET model. ASET is a model for the upper layer height and temperature in a single room fire. These two variables can be used to estimate the available safe egress time for a single room 
fire. In the original formulation, roughly a dozen physical parameters were reduced to four dimensionless core parameters. The current effort has further reduced that set to two core parameters. The primary physical parameters are:

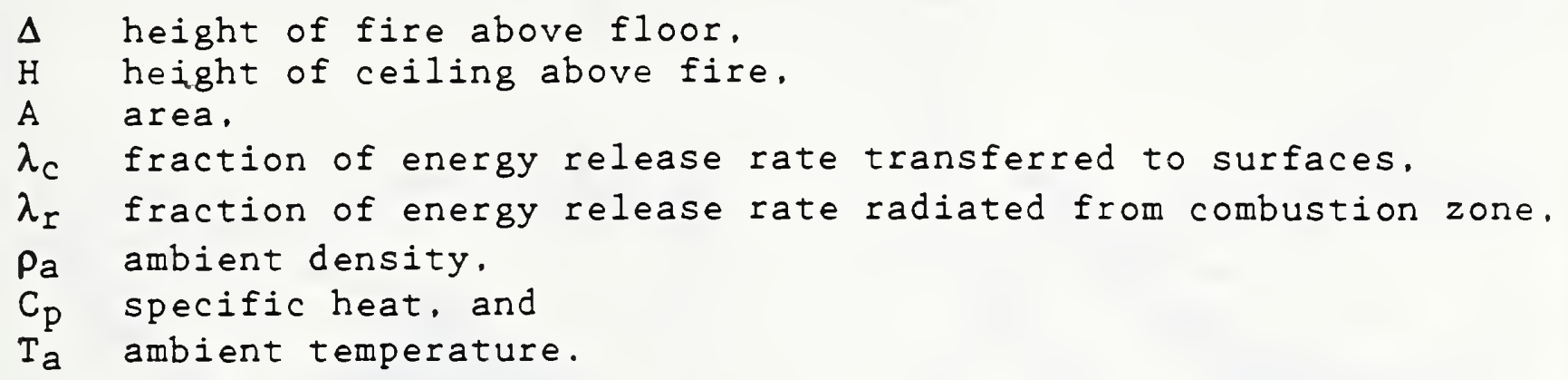

These are combined to produce core parameters $\alpha_{1}$ and $\alpha_{2}$ where $\alpha_{1}=\Delta /(\Delta+H)$ and

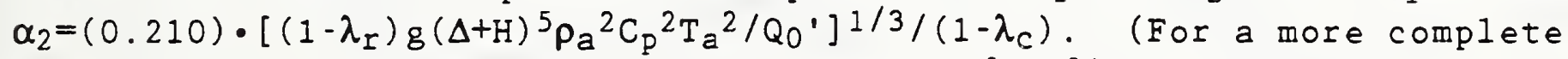
description of the physical parameters, see [1,2]).

To illustrate the advantages of the core parameter representation, we consider the effects of changing two physical parameters in the ASET model: room height and area. Figure la shows how these two parameters might be ranged in order to apply the ASET model in a variety of conditions. Figure lb illustrates the same values of height and area when translated to the two core parameters. Note that the apparent uniform coverage of the physical parameter space illustrated in Figure $1 a$ is not actually realized when viewed in terms of the core parameters.

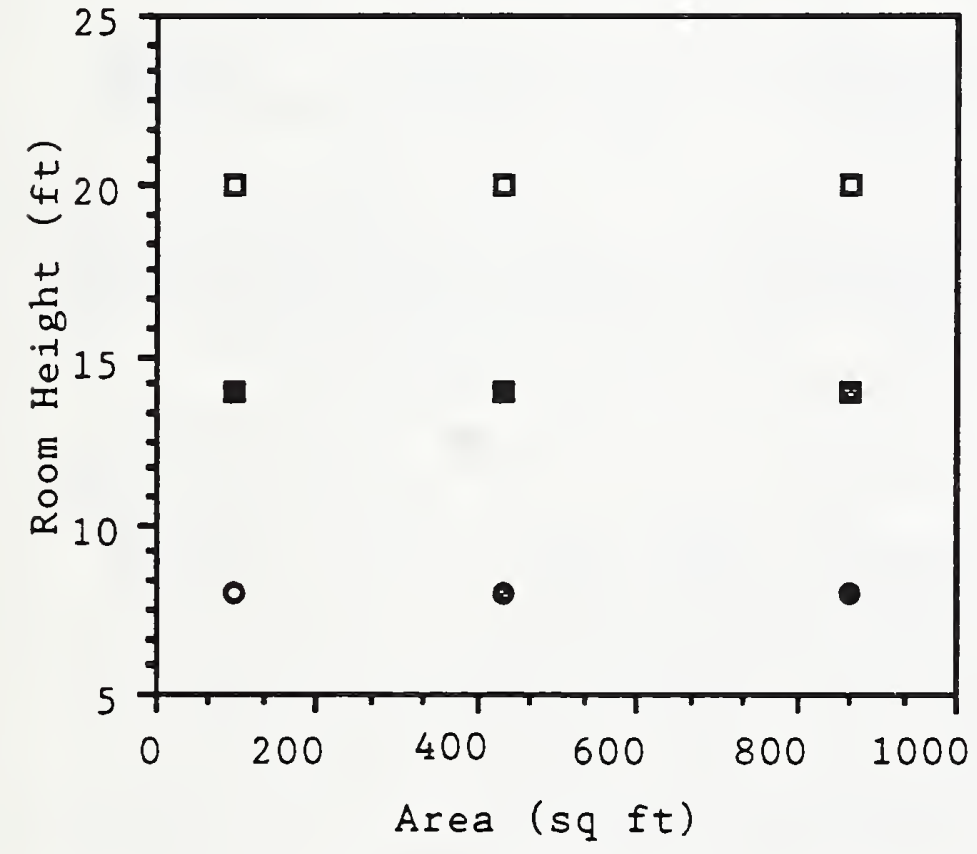

(a)

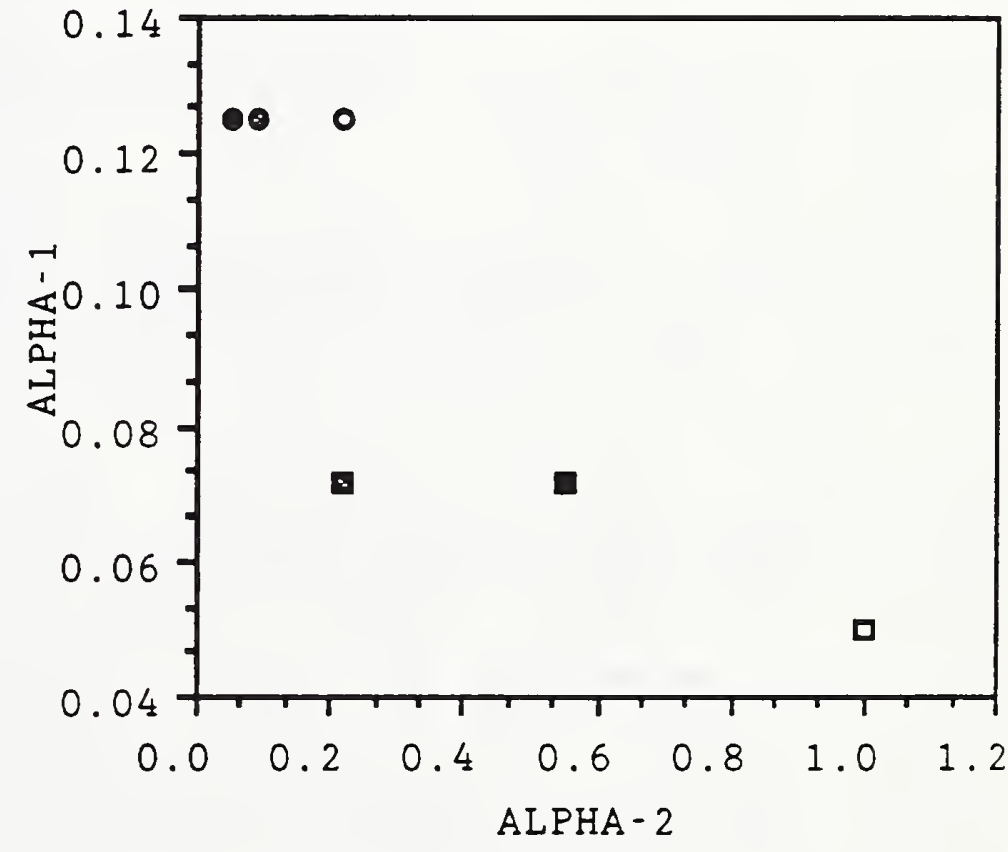

(b)

Figure 1 .

Correspondence between physical parameters (a) and associated core parameters (b).

Uniform coverage of the parameter space is accomplished using the core parameters. However, uniform coverage of the model's response surface might not be the most appropriate method of analysis. As illustrated in Figure 2a, only certain values in the core parameter space might correspond to realizable physical parameters. In addition, in describing the response surface of a 
model, it is more efficient to utilize a few points in a region where the response surface is uniform with a larger number of points where the model's output changes more rapidly (Figure 2b). All of these techniques can be applied to a model to provide greater efficiency in computation and greater understanding of the model's output.

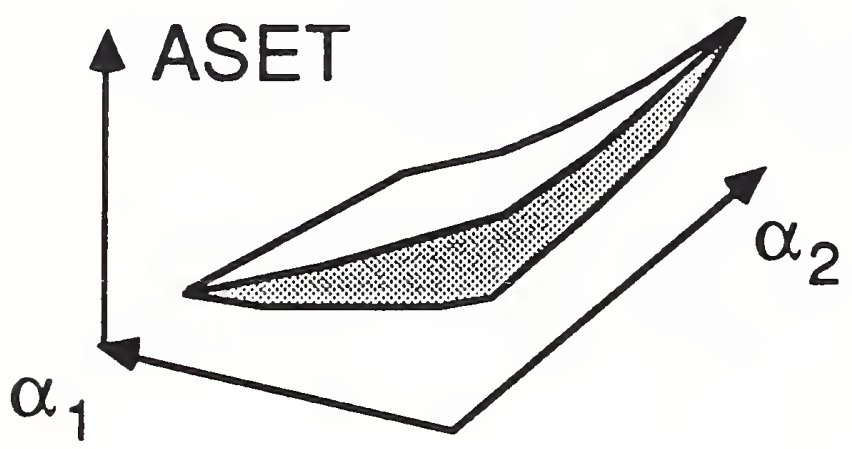

(a)

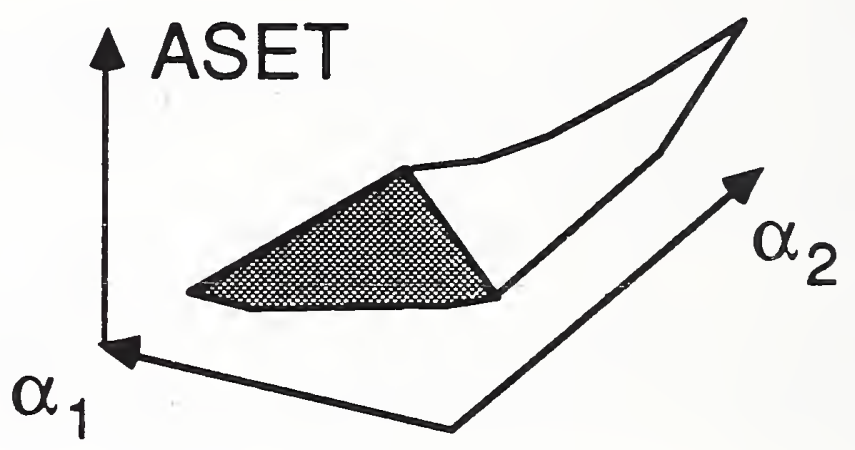

(b)

Figure 2.

(a) Non-rectilinear region in core parameter space corresponding to physically realizable parameters.

(b) Regions of uniform versus rapid change in model output.

Sensitivity Analysis. Sensitivity analysis for a model generally involves identification of dominant variables. feasible output range. and the output range generated by a specified input range. Specifically. this information is used in determination of the accuracy required of input variables. the number of input variables to be studied, and the basis of comparison between model and experimental data.

For a model having an $n$ dimensional state vector and $a \mathrm{k}$ dimensional parameter vector. the sensitivity analysis problem can be stated as an estimation problem for the nk "sensitivity coefficients" - the time-varying partial derivatives of the output vector with respect to the input parameters. Several approaches for estimating these sensitivity coefficients have been investigated using the ASET model. These include finite differences and both direct coupled and decoupled methods [3.4]. The use of finite differences is fairly standard and will not be discussed here. The direct coupled method combines the original system of $\mathrm{n}$ differential equations with an additional nk differential equations (one for each sensitivity coefficient) and solves the resulting system directly. The direct decoupled method involves solving the original system of $n$ differential equations and then using an interpolation scheme to estimate the sensitivity coefficients from the time-dependent output vector. For ASET, the latter is equivalent to solving $k$ systems of $n$ linear equations.

Figures $3 a$ and $3 b$ illustrate the results obtained via the direct decoupled approach as applied to ASET. These figures show the dependence and sensitivity of both upper layer height and temperature to a $10 \%$ variation in room height and area respectively. Again. typical sensitivity analysis information is obtained: in this case. the output variables are relatively insensitive to changes in room height (Figure $3 a$ ) and the upper layer temperature is fairly sensitive to changes in room area (Figure $3 \mathrm{~b}$ ). 


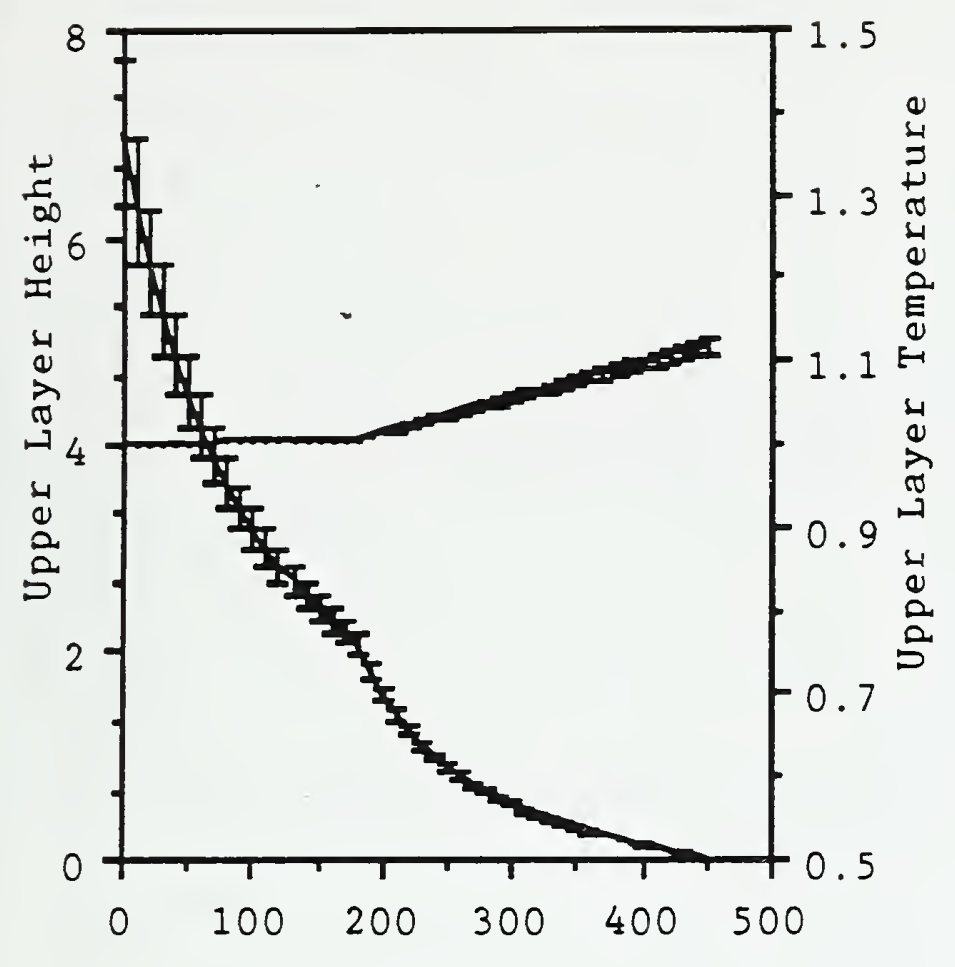

Time

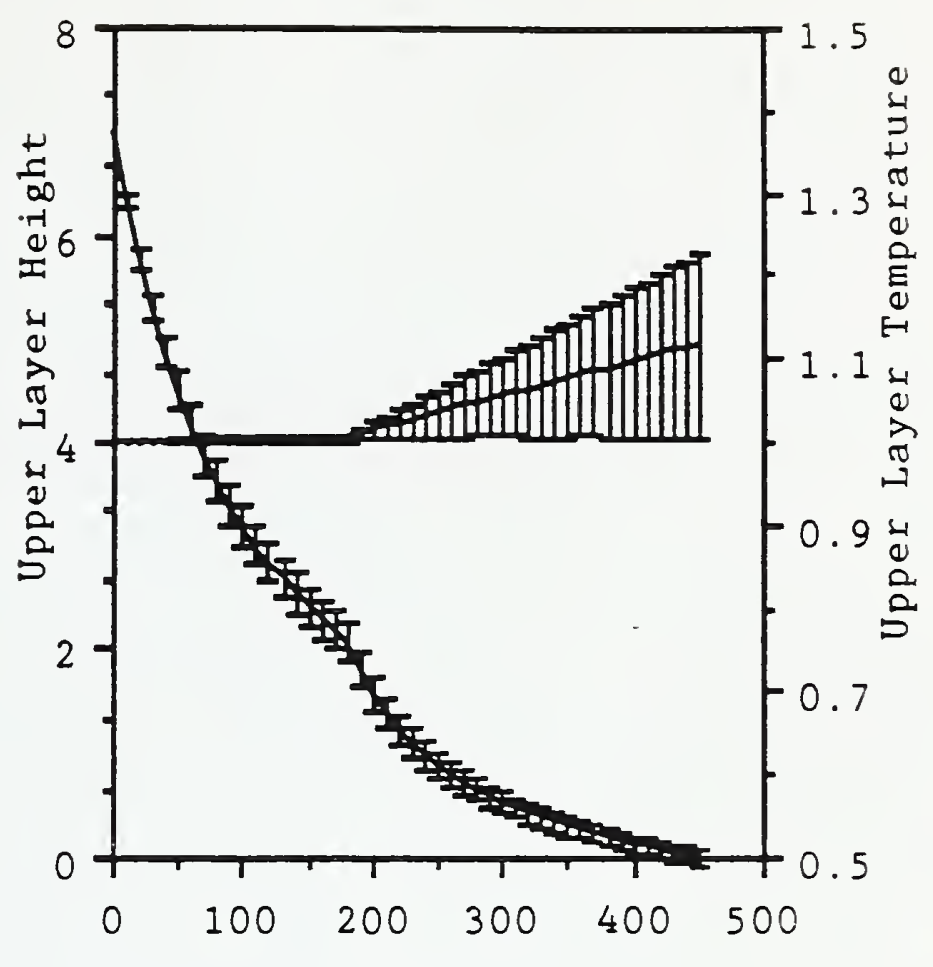

Time

(a)

(b)

Figuré 3 .

(a) Change in upper layer height/temperature with a $10 \%$ change in room height.

(b) Change in upper layer height/temperature with a $10 \%$ change in room area.

Summary. A variety of techniques are being examined for their applicability to the fire validation problem in the context of the ASET model. Future areas for examination include incorporation of the use of core parameters into the sensitivity analysis. further comparison of the advantages of the different approaches to sensitivity analysis, and combining these tools and techniques into procedures and software to enhance their more general applicability.

\section{References}

1. Cooper. L. Y.. "A Mathematical Model for Estimating Available Safe Egress Time in Fires." Fire and Materials 6, 135-142 (1982).

2. Cooper. L. Y. and Stroup. D. W. "ASET-A Computer Program for Calculating Available Safe Egress Time." Fire Safety Journal 9, $29-45$ (1985).

3. Dickinson, R. P. and R. J. Gelinas, "Sensitivity Analysis of Ordinary Differential Equation Systems - A Direct Method," Journal of Comp. Physics 21. $123-143(1976)$.

4. Dunker. A. M., "The Decoupled Direct Method for Calculating Sensitivity Coefficients in Chemical Kinetics," I. Chem. Phys, 81-5, 2385-2393 (1984).

\section{Reports and Papers:}

J.P. Jarvis, M.M. Kostreva, and C.L. Forney, "Tools for Validation and Analysis of Fire Models," Combined Proceedings, 20 th Fall Technical Meeting of the Eastern Section of the Combustion Institute \& Annual Conference of the Center for Fire Research. November 1987. 
PART I. Basic Research and Tools for "Engineered" Fire Safety (In-House Projects and Associated Grants Funded by NIST)

D. HAZARD MODELING 



\section{Professional Personnel}

William M. Pitts, Project Leader

George W. Mulholland, Head, Smoke Dynamics Group

Nelson P. Bryner, Chemical Engineer

\section{Project Objective}

Develop scientifically-sound principals, metrology, experimental data, and calculational procedures for measurement and prediction of carbon monoxide (CO) production by fires and to utilize this information to assess the hazard due to this toxic species.

\section{$\underline{\text { Scope }}$}

This program is designed to assess the importance of $C O$ in fire toxicology and to provide the scientific background required to allow the prediction of $\mathrm{CO}$ in real fires. Efforts range from purely empirical studies such as assessments of $C O$ production in full scale fire tests to fundamental studies designed to improve the understanding of the chemically reacting turbulent flows which ultimately produce $C O$. New information which is generated is incorporated into existing CFR models of fire behavior.

\section{Technical Accomplishments}

\section{CO Production in Full-Scale Enclosure Fire Tests}

Results of several series of full-scale fire tests have been analyzed to assess the concentrations of $\mathrm{CO}$ produced by large enclosure fires. The production of $\mathrm{CO}$ is characterized by the $\mathrm{CO} / \mathrm{CO}_{2}$ ratio. The importance of ventilation parameter on the burning behavior is clear. If a fire is fully ventilated the $\mathrm{CO} / \mathrm{CO}_{2}$ ratio is generally low $(<0.01)$. However, as the fire becomes underventilated $\mathrm{CO}$ levels increase greatly and the $\mathrm{CO} / \mathrm{CO}_{2}$ ratio can approach 0.3. The final levels of Co detected for positions well away from underventilated fires appear to depend on whether or not additional burning of combustion products occurs outside of the enclosure. The conditions necessary for additional burning are not well characterized and this lack of knowledge probably represents the greatest uncertainty concerning the conditions necessary for the production of high $C O$ concentrations at positions far from a fire source.

\section{Effects of Vitiation on Production of $\mathrm{CO}$}

The cone calorimeter is widely utilized for investigating the combustion of small fuel samples under conditions of heat irradiation characteristic of large fires. Combustion products produced are collected and analyzed. The $\mathrm{CO} / \mathrm{CO}_{2}$ ratio is one of the parameters which is determined. In the past, measurements have been performed under fully ventilated conditions which generally produce low levels of $\mathrm{CO}$. Modifications are in progress which will 
allow control of the amount and composition of the "air" provided to the combustion region of the cone calorimeter. The new design features include an adjustable total flow through the chamber with flow straightening, variable $\mathrm{O}_{2}$ concentration, load cell separated from combustion chamber, overpressure panel on back of cone, and a radiation shield for the specimen. In this way it will be possible to investigate the production of $\mathrm{CO}$ for underventilated and partially-vitiated conditions. Systematic studies utilizing this modified cone calorimeter will provide new insights into the conditions which result in the formation of high concentrations of $\mathrm{CO}$ in fires. Wood has been chosen as the first material to be investigated because of its wide use and because of the known effect of reduced ventilation on combustion product yields. Large scale tests performed by Rasbash at the Fire Research Station and by Williamson at Berkeley indicate that both $\mathrm{CO}$ and smoke yield increase by more than a factor of ten as the ventilation rate is decreased.

\section{Fundamental Investigations of Turbulent Combustion}

The production of $\mathrm{CO}$ is ultimately due the entrainment, mixing behavior, and chemical reactions occurring within the turbulent buoyant plume of a fire. Due to the heat release there is a strong coupling of the reactions occurring in the fire and the turbulent structure of the plume. This part of the CO priority project is an effort to characterize the turbulent structures responsible for mixing within buoyancy-driven plumes. Measurements are made in isothermal, buoyancy-driven flows generated by using gases which are lighter than air (e.g., helium or methane). During the past year flow diagnostics developed for multi-point measurements of concentration in buoyant plumes have been significantly improved by modifying existing equipment and improving data acquisition capabilities. A cylindrical enclosure having a height and diameter of $2.4 \mathrm{~m}$ has been designed and is under construction. It is to be utilized to provide a well-characterized environment for the characterization of the buoyant flows.

\section{Reports and Publications}

"Development of a Line Camera for Real-Time Measurements of Concentration in Turbulent Flow Fields, W. M. Pitts, Proceedings of the Fifth International Congress on Applications of Lasers and Electroptics, Volume 58, The Laser Institute of America, 1987, pP. 7-14.

"The Effects of Global Density and Reynolds Number Variations on Mixing in Turbulent, Axisymmetric Jets--Implications for Turbulent Jet Diffusion Flames,"W. M. Pitts, Proceedings of the 1987 ASME/JSME Thermal Engineering Joint Conference, Vol. 1, Eds. P. J. Marto and I. Tanasawa, The American Society of Mechanical Engineers, 1987, pp. 123-135.

"Mixing in Variable Density, Isothermal Turbulent Flows and Implications for Chemically Reacting Turbulent Flows," W. M. Pitts and T. Kashiwagi, National Bureau of Standards Internal Report, NBSIR 87-3550, May, 1987.

\section{Related Grants}

"Soot Particle Formation and Destruction in Diffusion Flames," Robert J. Santoro, Pennsylvania State University. 
"Radiation From Turbulent Luminous Fires," Gerald M. Faeth, University of Michigan.

"Experimental Studies of the Environment and Heat Transfer in a Room Fire," Edward E. Zukoski, California Institute of Technology. (See p. 97 for description) 


\title{
CENTER FOR FIRE RESEARCH \\ NATIONAL BUREAU OF STANDARDS \\ FY 88
}

Institution: The Pennsylvania State University

Grant No: $\quad$ 60NANB7D0706

Grant Title: Soot Particle Formation and Destruction in Diffusion Flames

\author{
Principal Investigator: $\quad$ Dr. Robert J. Santoro \\ Department of Mechanical Engineering \\ 313A Mechanical Engineering Building \\ University Park, PA 16802
}

$\begin{array}{ll}\text { Other Professional Personnel: } & \text { T. F. Richardson, Doctoral Student } \\ & \text { R. Puri, Doctoral Student } \\ \text { NBS Scientific Officer: } & \text { Dr. Kermit C. Smyth }\end{array}$

Technical Abstract:

Introduction The formation of soot particles has important effects on energy transfer and combustion product emissions from fires which dramatically impact loss of life and property. The objective of the present study is to provide a fundamental understanding of the soot formation and destruction processes which impact fire growth, spread and emissions.

The approach adopted emphasizes two elements of the problem. The first element concentrates on the processes responsible for the conversion of fuel carbon to soot (i.e. particle inception and surface growth) and particularly stresses the importance of fuel molecular structure. The second element examines the destruction stage of the process which reduces the soot concentration in the later parts of the flame. In particular, specific information on the relationship between soot and $\mathrm{CO}$ production is sought. A basic thrust of the work is to identify the overall mechanism relating the emission of $\mathrm{CO}$ and soot particles from flames. In addition, fundamental information on soot oxidation processes as well as the role of soot particles in the competition for oxidizing species such as $\mathrm{O}_{2}$ and $\mathrm{OH}$ may be forthcoming.

The results of this study are of direct relevance to the problems of fire research. Soot particle formation and destruction along with other basic phenomena such as ignition, hydrocarbon chemistry, turbulence, radiative transfer and plume dynamics, constitute the fundamental elements of the fire problem. Soot particles, once formed, are not passive species in the evolution of the fire situation. Through their radiative properties, soot particles can strongly enhance flame spread rates or the conditions necessary for "flash over" to occur. Soot particles present other complications through their role in visual obscuration and potential effects on carbon monoxide formation. These effects represent serious problems in terms of victim escape from fire situations and significantly contribute to fire mortality rates. However, with a proper understanding of the fundamental controlling mechanisms, significant improvements in the predictive capabilities of fire science modeling can dramatically improve the present situation. 
Experimental Approach These studies are carried out using a coannular diffusion flame facility to which extensive laser-based diagnostics can be applied for particle and velocity measurements. The burner, which has been previously described in detail [1], consists of a 1.1 $\mathrm{cm}$ diameter fuel tube surrounded by a $10 \mathrm{~cm}$ diameter air annulus. Flow conditioning of the air and fuel flows is provided by a series of glass beads and fine screens. A ceramic honeycomb at the exit of the air annulus is used to provide a uniform flow field. The fuel flow can consist of up to three metered gases which provides for the study of fuel mixtures as well as nitrogen dilution of the fuel for temperature control. To protect the flame from room disturbances, a metal chimney has been incorporated into the burner facility. This chimney translates horizontally with the burner, which slides vertically within the chimney. Slots machined in the chimney allow for optical access. The burner is mounted on a pair of computer controlled motorized stages which provide for vertical and horizontal motion. These motorized stages are used to traverse the burner through the laser beam to obtain measurements over the cross section of the flame at a particular height.

In order to accomplish the objectives of this study, a number of measurement capabilities are required. These include laser light scattering for particle sizing, laser velocimetry for velocity measurements and intrusive probing for temperature and species concentration measurements. Figure 1 shows a schematic representation of the laser scattering/extinction system used to characterize the soot particle field throughout the flame. In addition to the single scattered light detector shown in this figure, light scattering measurements can also be obtained at $45^{\circ}$ and $135^{\circ}$. Signal detection from the photodetectors is accomplished using separate computer controlled lock-in amplifiers. This approach allows for a high degree of user independent data acquisition and significantly eases the experimental procedures. A similar arrangement is used for the laser velocimetry measurements. A probe system to obtain $\mathrm{CO}, \mathrm{CO}_{2}$ and $\mathrm{O}_{2}$ concentration measurements is presently near completion.

Instrumentation for continuous measurement of these species is available using standard online techniques (e.g. NDIR for $\mathrm{CO}$ and $\mathrm{CO}_{2}$ measurements). In addition, gas chromatography and mass spectrometry techniques are available for more detailed measurements.

Technical Accomplishments The study of the effects of fuel molecular structure on the formation of soot is one of the major elements of the present work. However, a difficulty in studying fuel structure effects has been the wide differences in the flame size and shape which results when various fuels are burned at, for example, a characteristic condition such as the soot point. These differences result in important variations in the temperature and velocity fields which make quantitative comparisons impossible between the flames. In the present studies, this difficulty is addressed by adopting a fuel addition approach involving well characterized baseline flames in which the soot particle field is characterized in detail. The fuel molecular structure effects are then investigated by adding an additional fuel component to the baseline flame. However, the total carbon flow rate is held constant. This means that an appropriate fraction of the baseline fuel is replaced with the additive fuel. With the soot contribution from the baseline fuel known, the effect of the additive fuel on the formation of soot particles can be determined. Under these conditions, the flame size and shape remains similar for all flames.

Two fuels, methane and ethene, have been selected to serve as the baseline flames. The methane flames have relatively low soot particle formation and thus provide greater sensitivity for measuring changes introduced by varying the fuel molecular structure. The ethene flames represent the best studied set of diffusion flames presently available and provide a significant comparative data base [1-2]. Comparisons between the two baseline systems will also provide a basis for examining potential synergistic effects between the fuel addition species and the baseline fuel. Studies of the ethene and methane baseline flames have been completed and studies of a specific series of fuels have been initiated. These studies involve comparisons between an alkane, alkene and alkyne fuel added to a methane baseline flame. Specifically 
butane, 1-butene and 1,3-butadiene have been investigated.

These initial studies have concentrated on soot particle measurements using the laser scattering/extinction approach. Analysis of the results of these measurements indicates a significant increase in the amount of fuel converted to soot as the fuel added to the flame is varied. Based on the mass of fuel added, the fuel conversion percentage increases from $12 \%$ for butane to $35 \%$ and $44 \%$ for butene and butadiene, respectively, at the location of maximum soot concentration in the flame. A more detailed comparison is given in Fig. 2 where the integrated soot volume fraction is plotted as a function of axial location. The integrated soot volume fraction, $F_{v}$, is given by

$$
F_{v}=2 \pi \int_{0}^{R} f_{v}(r, z) r d r
$$

where $f_{v}$ is the soot volume fraction at $r$ and $z$, the radial and axial positions respectively. Thus, $F_{\mathrm{V}}$ represents a measure of the total amount of soot at a particular axial position. These results clearly indicate a systematic decrease in the axial position where soot is first observed as a more sooty fuel is introduced into the flame. This indicates that the reactions leading to the first soot particles occur more rapidly in these flames. More detailed comparisons utilizing the velocity and temperature fields should offer the potential for comparison with overall chemical kinetic rate information describing the pyrolysis and oxidative pyrolysis mechanisms established for these fuels. Recent work using probe sampling techniques have demonstrated that such comparison approaches are quite promising [3].

The results shown in Fig. 2 are limited to regions of the flame where particles are present. As an approach to investigating the preparticle and inception regions of the flame, laser fluorescence measurements have also been obtained. These measurements can be viewed to be indicative of the presence of large hydrocarbon species which may be involved in the particle inception chemistry. The objective of these studies is to provide a relative measure of the hydrocarbon chemistry which can be compared to more fundamental chemical modeling approaches [3].

In addition to providing a basis from which to examine fuel molecular structure effects on soot particle inception and growth, these studies will be used to examine the effects of soot concentration on $\mathrm{CO}$ emission from these flames. As the previous results demonstrate, a wide variation in soot loading can be achieved through appropriate fuel addition.

Measurements of the $\mathrm{CO}$ concentration will be made above these flames in order to assess the effect of soot loading on the $\mathrm{CO}$ emission from fires.

The significance of these results lies in the potential to provide fundamental insights into the soot formation and destruction chemistry. Additionally, such data can be used to identify the important processes controlling actual fire situation. From these insights, approaches to handling the more complicated multicomponent fuel conditions of real fires can be addressed.

\section{$\underline{\text { References }}$}

1. Santoro, R. J., Semerjian, H. G. and Dobbins, R. A., Combustion and Flame, 51, p. 208 (1983).

2. Santoro, R. J., Yeh, T. T., Horvath, J. J. and Semerjian, H. G., Combustion Science and Technology, 53, p. 89 (1987). 
3. Miller, J. H., Mallard, W. G., and Smyth, K. C., Twenty-First Symposium (International) on Combustion, The Combustion Institute, pp. 1057-1065 (1986).

\section{Reports and Papers}

R. J. Santoro and H. G. Semerjian, "The Effect of Fuel Structure on the Formation and Growth of Soot Particles in Diffusion Flames", The Twenty Eighth Biennial Conference on Carbon, Worcester Polytechnical Institute, (July 18-24, 1987), Worcester, MA.

R. J. Santoro, "Soot Particle Formation in Diffusion Flames", American Chemical Society Meeting, Advances in Soot Chemistry, (Aug. 30-Sept. 4, 1987), New Orleans, LA.

R. J. Santoro, "Fuel Molecular Structure Effects on Soot Particle Growth in Diffusion Flames", Eastern Section Meeting of the Combustion Institute, Twentieth Fall Technical Meeting, Nov. 2-5, 1987, Gaithersburg, MD.

Laser Scattering/Extinetion System

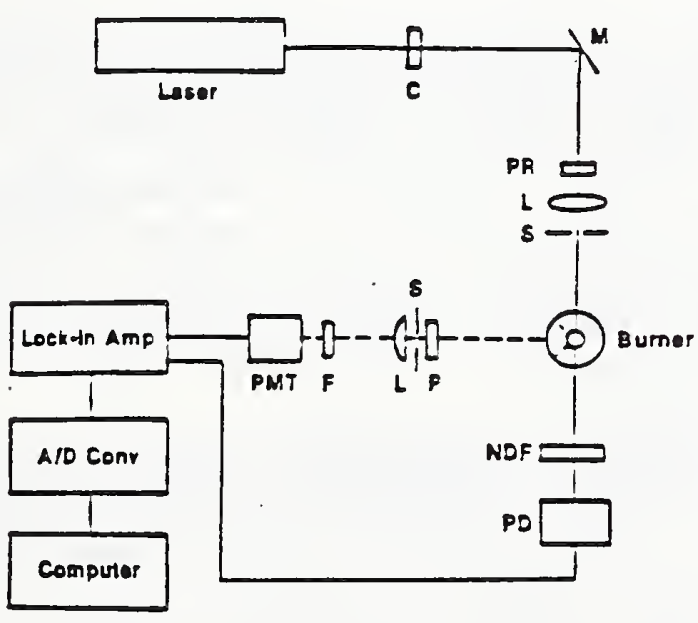

C - ehoppor, M - mirror, PR - polartzation rotator, L - Lns, 8 - spatial niter NDF - neotral dansity filer, PD - photodiode, P - pobrizar, F - natrow band niter, PMT - photomulisplier,

Fignre 1 Scbematic of leser scatrering/extinction apparatus.

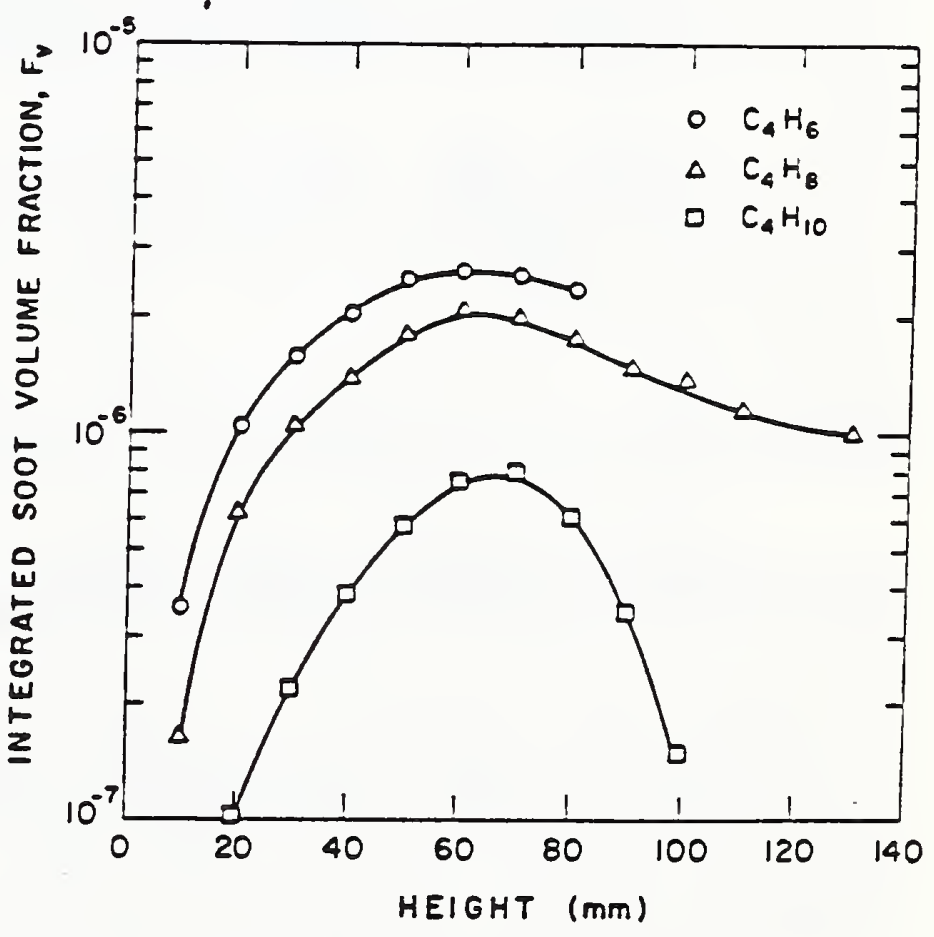

Figere 2 Comparisos of the integrated soo: volume fraction. Fu, for the buane, 1-butene and 1.3-buadiene fuel addiuoo studies. The flames had a methape flow rate of $5.6 \mathrm{~cm}^{3} / \mathrm{s}$ and a fuel addition fion nite of $1.05 \mathrm{~cm}^{3} / \mathrm{s}$. 


\section{CENTER FOR FIRE RESEARCH \\ NATIONAL BUREAU OF STANDARDS \\ FY 88}

Institution:

Grant No:

Grant Title:

Principal Investigator:

Other Professional Personnel:

NBS Scientific Officer:
The University of Michigan

60NANB5D0576

Radiation from Turbulent Luminous Flames

Professor Gerard M. Faeth

Department of Aerospace Engineering

218 Aerospace Engineering Building

Ann Arbor, MI 48109-2140

M. A. Kounalakis, Graduate Assistant

Y.R. Sivathanu, Graduate Assistant

Dr. David D. Evans

\section{Technical Abstract:}

Introduction. This investigation is considering two aspects of thermal radiation from unwanted fires, as follows: (1) the scalar structure of turbulent flames, emphasizing the radiation properties of soot in luminous flames; and (2) effects of turbulence/radiation interactions, emphasizing nonluminous flames to simplify interpretation of results. The findings have application to modeling fires in structures, developing materials test codes, and developing fire detectors. Progress on the two phases is briefly discussed in the following, additional details can be found in the papers and reports listed and the end of this report.

Luminous Flames. The laminar flamelet concept of turbulent nonpremixed flames implies that scalar properties can be correlated solely as functions of mixture fraction, called state relationships, independent of local values of flame stretch and the past history of the flow. When this concept applies, predictions of the scalar structure of turbulent flames are vastly simplified since only governing equations for mixture fraction properties must be solved. The laminar flamelet concept is widely accepted for gas species concentrations in turbulent diffusion flames except near points of flame attachment where effects of finite-rate chemistry prevail. The objective of the present investigation is to determine the conditions, if any, where the concept can be extended to soot volume fractions - the soot property that primarily influences thermal radiation.

Measurements of soot volume fraction state relationships are being undertaken for a variety of burner sizes (5, 50 and $250 \mathrm{~mm}$ diameter), fuel flow rates, and fuels. Work thus far has been limited to the overfire region. Figure 1 is an illustration of the soot volume fraction state relationship measured for acetylene/air diffusion flames. The data represents measurements of soot volume and mixture fractions at various points in the overfire region of these flames. The correlation in the rich region is based on earlier measurements in laminar flames, and in the lean region by assuming a constant soot generation efficiency (defined as the fraction of fuel carbon converted to soot). The correlation in the lean region implies that soot reaction quenches at a fixed soot volume and mixture fraction, followed by passive mixing in the overfire region. The measurements agree quite closely with the correlation, implying a wide range of conditions where the laminar flamelet concept for soot volume fractions can be used for radiation analysis of acetylene flames.

Tests have also considered propylene, ethylene and propane flames. It has been found that soot volume fraction state relationships become progressively less satisfactory as the propensity of the fuel to soot decreases. In effect, the soot generation efficiency appears to be constant for a given flame conditions but the soot generation efficiencies tend to increase as flow residence times in the flame increase. These results are illustrated in Fig. 2, where soot generation efficiencies are plotted as a function of estimated characteristic residence times for all the fuels. Clearly, soot generation efficiencies are relatively independent of residence time (for the range tested) for acetylene, which accounts for the reasonably good soot volume fraction state relationship seen in Fig. 1. However, the other fuels exhibit residence time effects - particularly when their soot generation efficiencies are low - although soot generation efficiencies appear to approach an asymptotic value at long residence times. If this proves to be true, the laminar flamelet concept for soot volume fractions would still be a viable approach for treating a wide range of practical fires. 
Current work is extending measurements to the $250 \mathrm{~mm}$ diameter bumer so that the tendency to reach asymptotic values of soot generation efficiencies at long residence times can be studied. Direct measurements of characteristic residence times are also being undertaken so that conclusions will not be based on uncertain estimates. The next phase of the work will involve evaluating the laminar flamelet concept for soot volume fractions in the fuel-rich region of turbulent diffusion flames.

Nonluminous Flames. Past work has shown that radiation from turbulent flames is generally several times larger than estimates based on mean scalar properties within the flames. This is an effect of turbulence/radiation interactions, i.e., the fact that radiation properties are a very nonlinear function of scalar properties. Several ad hoc stochastic methods have been developed, which have yielded encouraging predictions of effects of turbulence/radiation interactions. The objective of present work is to extend these concepts, improving their physical basis and providing methods for predicting the properties of radiation fluctuations.

Experiments concerning radiation properties are being undertaken in round turbulent hydrogen/air and carbon monoxide/air flames. Measurements include mean and fluctuating spectral radiation intensities for chord-like paths through the flames. Predictions are based on stochastic simulation of mixture-fraction variations along the radiation paths, seeking to simulate probability density functions and spatial and temporal correlations of mixture fraction fluctuations along the path. Given the mixture fraction distribution along the path at any instant, the state relationships yield all other scalar properties and a narrow-band radiation analysis yields the spectral radiation intensity. Continuing the simulation in time provides predictions of the temporal variation of spectral radiation intensity. Thus far, only a first-order temporal and spatial simulation has been studied, with points along the radiation path spaced at the integral length scale and being statistically independent. This approach yields reasonably good predictions of mean and fluctuating spectral radiation intensities.

Typical predictions and measurements for hydrogen/air flames are illustrated in Figs. 3 and 4. These results are for horizontal radiation paths passing through the flame axis. Figure 3 is an illustration of predicted and measured probability density functions of radiation fluctuations: predictions are quite encouraging, including correctly representing the increased skewness of the distribution with increasing height above the burner. Figure 4 is an illustration of power spectra of radiation fluctuations. These predictions are also encouraging except for overestimation of the high frequency portion of the spectra due to use of a simple first-order simulation.

Currently, measurements of radiation fluctuations are being undertaken for chord-like paths at various distances from the axis and the stochastic analysis is being extended to higher orders. Subsequent work will involve measurements of the statistical properties of mixture fraction along radiation paths in the flames, so that uncertainties of predictions of these properties can be avoided while the stochastic method is being developed.

\section{Reports and Papers:}

1. M.-C. Lai and G. M. Faeth, "Turbulence Structure of Vertical Adiabatic Wall Plumes," L. Heat Transfer, Vol. 109, No. 3, pp. 663-670, 1987.

2. J. P. Gore and G. M. Faeth, "Structure and Spectral Radiation Properties of Turbulent Ethylene/Air Diffusion Flames," Twenty-First Symposium (International) on Combustion, The Combustion Institute, Pittsburgh, PA, pp. 1521-1531, 1986.

3. G. M. Faeth, J. P. Gore, S. G. Chuech and S.-M. Jeng, "Radiation from Turbulent Diffusion Flames," Ann. Rev. Num. Fluid Mech. and Heat Trans. Hemisphere Publishing Corp., Washington, in press.

4. J. P. Gore and G. M. Faeth, "Structure and Radiation Properties of Luminous Turbulent Acetylene/Air Diffusion Flames," L. Heat Trans, Vol. 110, No. 1, pp. 173-181, 1988.

5. Y. R. Sivathanu, J. P. Gore and G. M. Faeth, "Scalar Properties in the Overfire Region of Sooting Turbulent Diffusion Flames," Combust. Flame, in press.

6. G. M. Faeth, J. P. Gore and Y. R. Sivathanu, "Radiation from Soot-Containing Flames," 70th AGARD Symposium on Combustion and Fuels in Gas Turbine Engines, NATO, Paris, in press.

7. M. E. Kounalakis, J. P. Gore and G. M. Faeth, "Turbulence/Radiation Interactions in Nonpremixed Hydrogen/Air Flames," Twenty-Second Symposium (International) on Combustion, The Combustion Institute, Pittsburgh, in press. 
8. J. P. Gore, M. E. Kounalakis and G. M. Faeth, "Temporal Properties of Radiation Fluctuations from Nonluminous Turbulent Diffusion Flames," Proceedings of the Twentieth Fall Technical Meeting, Eastern Section of the Combustion Institute, Pittsburgh, pp. 71.1-71.4, 1987.

9. M. E. Kounalakis, J. P. Gore and G. M. Faeth, "Turbulence/Radiation Interactions in Nonluminous Diffusion Flames," Proceedings of the 1988 Spring Technical Meeting, Central States Section of the Combustion Institute, Pittsburgh, pp. 135-140, 1988.

10. Y. R. Sivathanu, J. P. Gore and G. M. Faeth, "Soot Properties in the Overfire Region of Turbulent Diffusion Flames," Proceedings of the 1988 Spring Technical Meeting, Central States Section of the Combustion Institute, Pittsburgh, pp. 275-280, 1988.

11 G. M. Faeth, "Heat Transfer in Fires and Combustion Systems," National Heat Transfer Conference, Houston, TX, in press.

12. M. E. Kounalakis, J. P. Gore and G. M. Faeth, "Mean and Fluctuating Radiation Properties of Nonpremixed Turbulent Carbon Monoxide/Air Flanes," ASME Winter Annual Meeting, Chicago, IL, submitted.

13. Y. R. Sivathanu, M. E. Kounalakis, J. P. Gore and G. M. Faeth, "Radiation from Turbulent Nonluminous and Luminous Diffusion Flames," Annual Report, NBS Grant No. 60NANB5D0576, October 1987. 


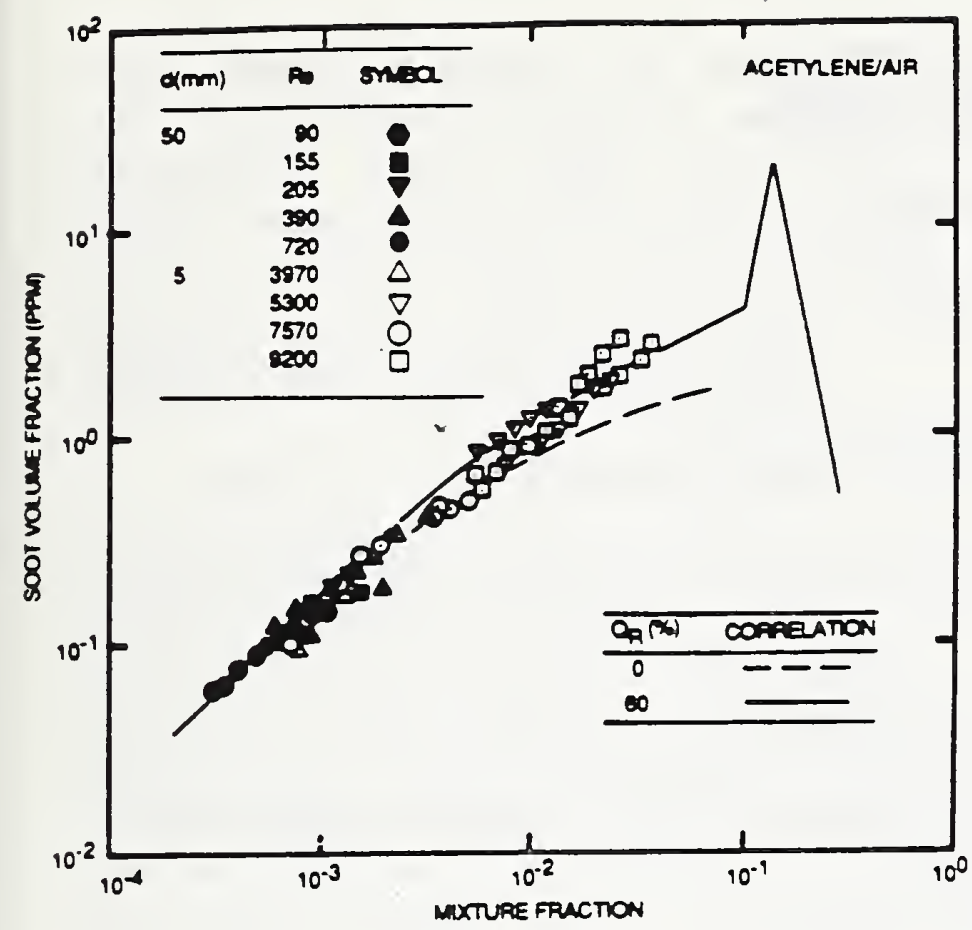

Fig. 1 Soot volume fraction state relationship in the overfire region of acetylene/air diffusion flames.

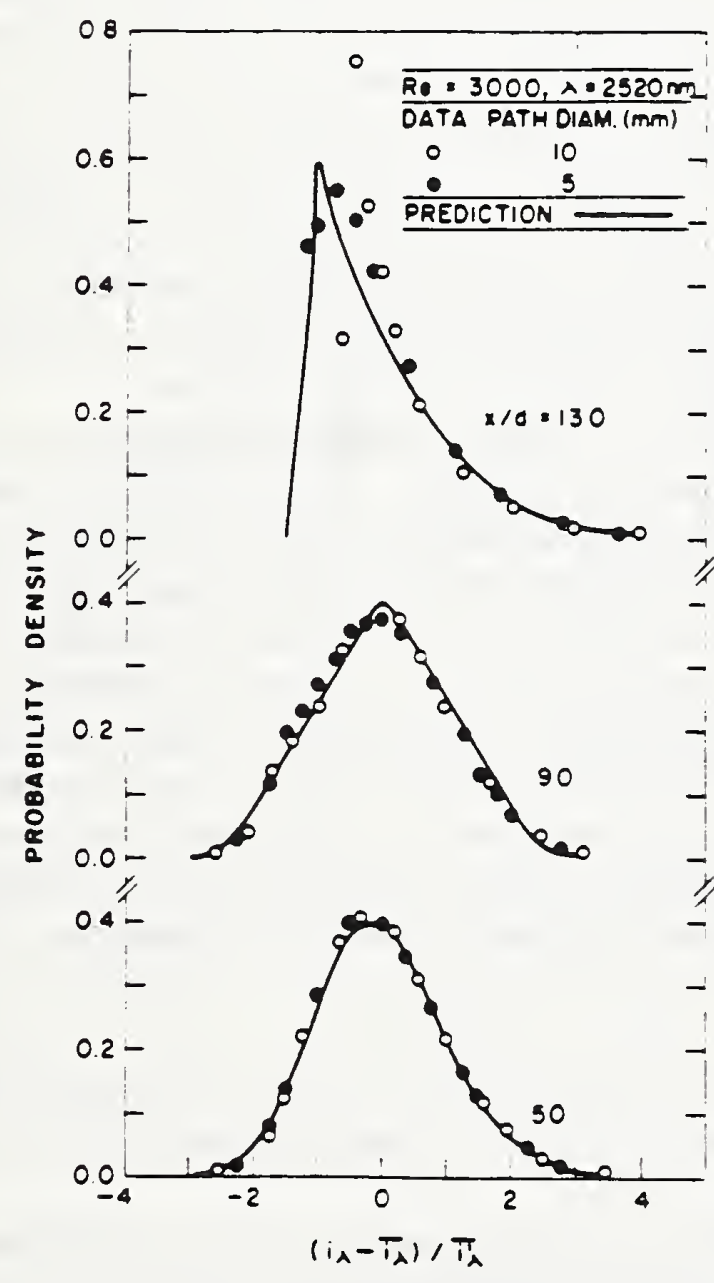

Fig. 3 Probability density functions of radiation fluctuations in a hydrogen/air diffusion flame.

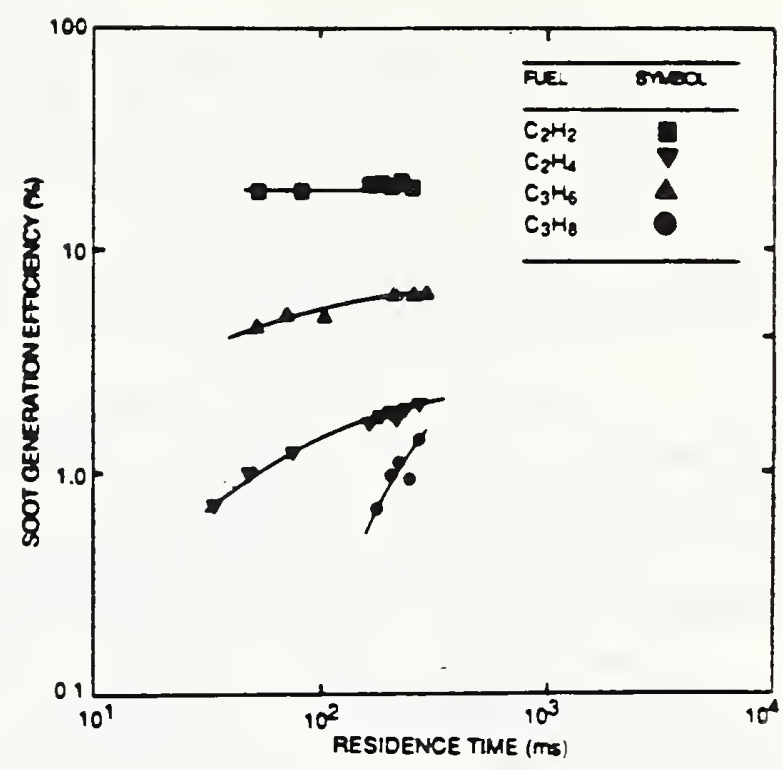

Fig. 2 Soot generation efficiencies as a function of residence time in hydrocarbon/air diffusion flames.

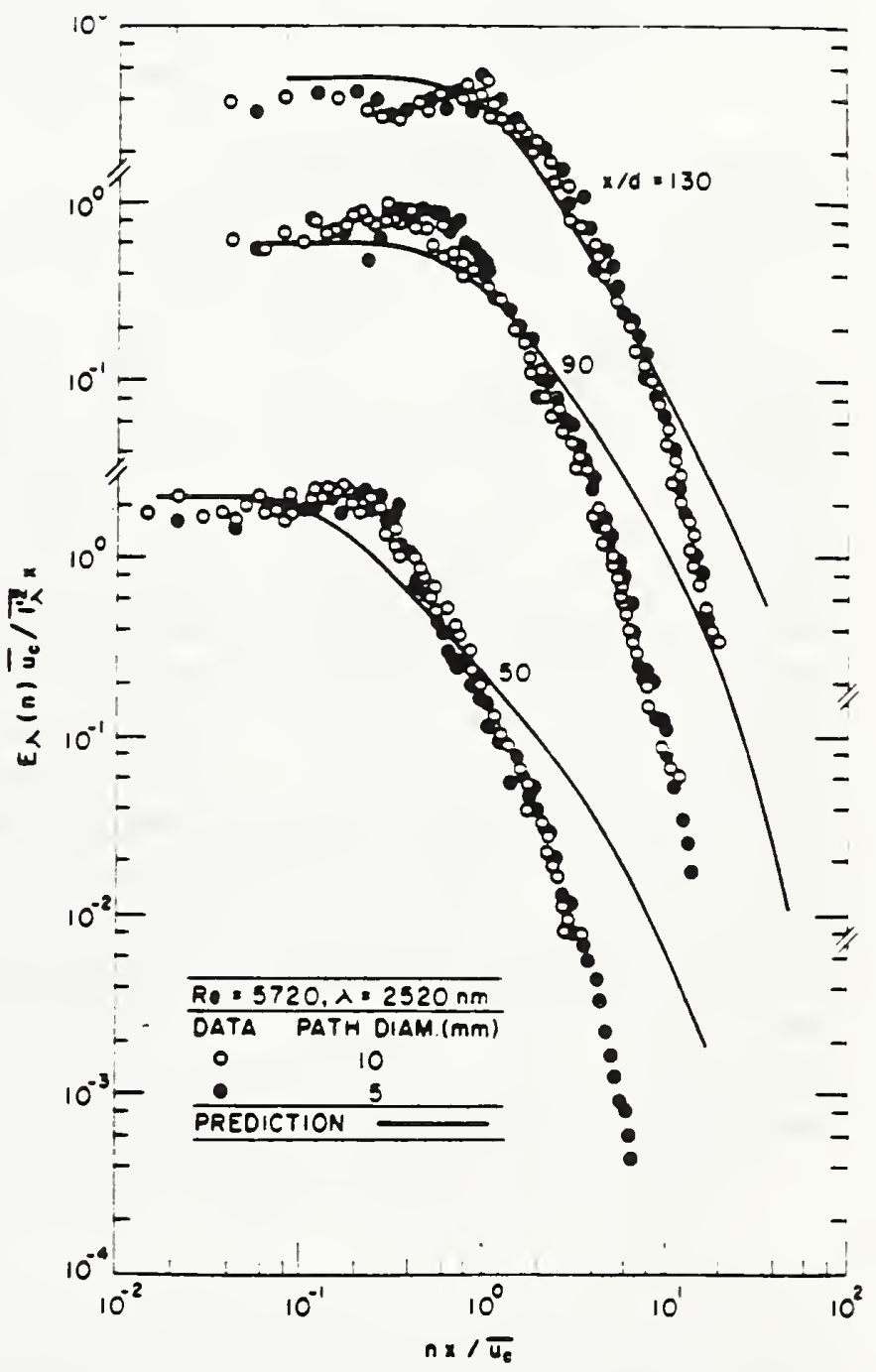

Fig. 4 Power spectra of radiation fluctuations in a hydrogen/ air diffusion flame. 


\section{CENTER FOR FIRE RESEARCH \\ PRIORITY PROJECT - 1988 \\ SMOKE TRANSPORT}

\section{Professional Personnel}

John H. Klote, Project Leader

Leonard Y. Cooper, Head, Building Fire Modelling Group

Kevin Greenaugh, Engineer

William Rinkinen, Technician

Walter Jones, Computer Scientist

Project Objective

Develop physical and mathematical models to predict the motion of smoke in buildings due to all significant mechanisms.

$\underline{\text { Scope }}$

This program studies the motion of smoke in buildings due to any driving force such as stack effect, wind effect, forced ventilation systems, and expansion and buoyancy of fire gases. Studies are by full scale and scale model experiments with initial emphasis on the influence of stack effect. The information generated by this effort is to be used to develop mathematical models suitable for incorporation in existing fire models.

\section{Technical Accomplishments}

\section{Report on Stack Effect}

In building fires, smoke often migrates to locations remote from the fire space. Stairwells and elevator shafts frequently become smoke-logged, thereby blocking evacuation and inhibiting fire fighting. In this report the following driving forces of smoke movement are discussed: stack effect, buoyancy of combustion gases, expansion of combustion gases, wind effect, and elevator piston effect. The steady flow analysis of stack effect and some considerations of unsteady flow are addressed. Methods of evaluating the location of the neutral plane are presented for a number of common building leakage configurations. The capabilities and limitations of network models for evaluation of smoke movement do to stack effect are discussed. The steady flow network method is applied to situations of opened and closed doors and shaft vents likely to occur during firefighting. A number of generalizations which could be of use to fire fighters are presented. The information in this paper also is applicable to the migration of other airborne matter such as hazardous gases, bacteriological matter or radioactive matter in laboratories, hospitals, or industrial facilities.

\section{Project Plan for Full Scale Smoke Control and Movement Experiments}

A project plan was developed for full scale smoke control and smoke transport experiments to be conducted at the Plaza Hotel in Washington DC. Smoke control will be achieved by exhausting the fire zone and pressurizing surrounding zones, and by pressurizing stairwells. The smoke control systems 
were designed using the analysis methods in the NIST Smoke Control Handbook and the design pressures recommended in NFPA 92A. The effectiveness of these systems to limit smoke flow will be measured, and the interaction between smoke control and fire development will be studied. Experiments without smoke control will allow a relative evaluation of the effectiveness of smoke control. Also, the non-smoke control tests will be used to study smoke flow under stack effect conditions.

\section{Design and Construct Scale Model Set Up}

A scale model of a seven story building including the stairwell and elevator shaft has been designed and built in a laboratory, and a forced air heating and cooling system for the model is under design. Fires will be set under "winter" and "summer" stack effect conditions. Several of the walls of the model are of glass allowing video recording of the smoke flow up the stairwell and elevator shafts and on building floors. Adjustable openings in walls and floors simulate the building leakage paths to allow modeling of various building leakage areas including opened and closed doors. It is anticipated that Plaza Hotel experiments and these scale model experiments will provide an understanding of the mechanisms involved in such smoke flow, and that this understanding will be the basis of a mathematical model.

\section{Develop Computer Graphics for Smoke Flow in Multi-Story Buildings}

Computer simulation of smoke movement in multi-story buildings results in very large quantities of data to be studied and evaluated. To aid in this evaluation, a computer graphic routine was developed for displaying the results of zone model simulations of smoke flow in a seven story building. The routine can be used on IBM PC and compatible machines with CGA, EGA or VGA graphic capabilities. The building elevation is displayed, and the depths of smoke layers on floors and in a shaft are shown as the computer progresses through an analysis.

\section{Reports and Publications}

Quintiere, J.G., Scaling Applications in fire Research, International Symposium on Scale Modeling, July 18-22, 1988, Japan Society of Mechanical Engineers.

Klote, J.H., Project Plan for Full Scale Smoke Movement and Smoke Control Tests, Nat. Bur. Stand. (U. S.), NBSIR 88-3800, June 1988.

Klote, J.H., Fire Safety Inspection and Testing of Air Moving Systems, Nat. Bur. Stand. (U. S.), NBSIR 87-3660, November 1987.

Klote, J.H. and Budnick, E.K., The Capabilities of Smoke Control: Part I -. Fundamentals and Zoned Smoke Control, presented at SFPE tutorial at NFPA Meeting in Portland, OR, 10 November 1987.

Budnick, E.K. and Klote J.H., The Capabilities of Smoke Control: Part II -System Performance and Stairwell Pressurization, presented at SFPE tutorial at NFPA Meeting in Portland, OR, 10 November 1987. 
Klote, J.H., An Overview of Smoke Control Technology, ASHRAE Transactions, Vol. 94, Part 1, 1988, pp 1211-1222.

Klote, J.H., An Analysis of the Influence of Piston Effect on Elevator Smoke Control, Nat. Bur. Stand. (U. S.), NBSIR 88-3751, April 1988.

Klote, J.H. and Tamura, G.T., Experiments of Piston Effect on Elevator Smoke Control, ASHRAE Transactions, Vol 93, Part 2a, 1987,pp 2217-2228.

Tamura, G.T. and Klote, J.H., Experimental Fire Tower Studies on Mechanical Pressurization to Control Smoke Movement Caused by Fire Pressures, Proceedings of the 2nd International Symposium on Fire Safety Science, Tokyo, Japan.

Tamura, G.T. and Klote, J.H., Experimental Fire Tower Studies on Elevator Pressurization Systems for Smoke Control, ASHRAE Transactions, Vol. 93, Part II, 1987, pp 2235-2257.

\section{OA Sponers}

The work above was funded in part by the following sponsors:

American Society of Heating, Refrigerating, and Air-Conditioning Engineers, Inc., Atlanta, GA 30329

Bell Atlantic Telephone Company Arlington, VA 22201

New Jersey Bell Telephone Company Newark, NJ 07101

U.S. Fire Administration

Emmitsburg, MD

U.S. Veterans Administration

Washington, DC 20420

US West Incorporated

Denver, CO 80202 


\section{CENTER FOR FIRE RESEARCH \\ PRIORITY PROJECT - 1988}

\section{TOXIC POTENCY MEASUREMENT}

\section{Professional Personnel}

Barbara C. Levin, Project Leader

Maya Paabo, Research Chemist

Emil Braun, Physicist

Marc R. Nyden, Research Chemist

Richard H. Harris, Jr., Chemist

Helene M. Clark, Guest Worker, University of Pittsburgh

Lane Highbarger, Guest Worker, University of Pittsburgh

Nancy Eller, Guest Worker, University of Pittsburgh

\section{Project Objective}

To develop models and methods to predict and measure the combustion products that evolve from burning materials and to predict the impact of those products on living organisms.

\section{Scope}

1. Develop theoretical and analytical tools for prediction, identification, and quantitation of toxic agents in fire atmospheres.

2. Develop a simplified gas model to predict the toxicity of complex gas mixtures found in fire atmospheres.

3. Develop a bioanalytical screening test to determine whether the combustion products from materials are extremely toxic (i.e., low concentrations produce toxic effect) or unusually toxic (i.e., the toxicity of the combined major fire gases is not sufficient to account for the observed toxicity). This test is also designed to minimize the use of animals.

\section{Technical Accomplishments}

The Center for Fire Research has been developing a simplified gas model (known as the N-Gas Model) to predict the toxicity of the complex gas mixtures found in fire atmospheres. One of the advantages of this model is the reduction in the cost of and the dependence on animal testing without sacrificing test accuracy. Research on the N-Gas model has progressed from the determination of the toxicity of individual gases - $\mathrm{CO}, \mathrm{CO}_{2}, \mathrm{HCN}$, and reduced $\mathrm{O}_{2}$ - in Fischer 344 rats exposed for 30 minutes and observed for at least 14 days to the development of a four-gas model based on the combined toxicological results of these four gases. Other exposure times ranging from 1 to 60 minutes for single gases and 5 to 60 minutes for two gas combinations - CO plus $\mathrm{HCN}$ and $\mathrm{CO}$ plus $\mathrm{CO}_{2}$ - have also been examined. With the exception of the 5-minute $\mathrm{CO}$ plus $\mathrm{CO}_{2}$ experiments, the additivity and synergistic effects observed from the 30-minute animal exposures were observed at the other times. In the 5 minute $\mathrm{CO}$ and $\mathrm{CO}_{2}$ experiments, the $\mathrm{LC}_{50}$ value was lower than the 5 minute $\mathrm{LC}_{50}$ value for $\mathrm{CO}$ alone, but the 958 confidence limits overlapped. The current $\mathrm{N}$-gas model appears to explain the deaths observed at the 30 -minute 
$\mathrm{LC}_{50}$ values of 17 out of 24 different cases of material thermal decomposition. The other cases need further examination.

In the past year, experiments on the toxicity of the combined three and four gases were performed. In addition, studies were conducted to examine if the synergism of $\mathrm{CO}$ and $\mathrm{CO}_{2}$ seen at sublethal concentrations of $\mathrm{CO}$ and $\mathrm{CO}_{2}$ occurred as well at the $\mathrm{LC}_{50}$ concentration of $\mathrm{CO}$. The three-gas combinations were studied by exposing animals for 30 minutes to various concentrations of $\mathrm{CO}, \mathrm{CO}_{2}$, and reduced $\mathrm{O}_{2}$ (Table 1 ) or combinations of $\mathrm{CO}, \mathrm{CO}_{2}$, and $\mathrm{HCN}$ (Table 2). Additional experiments were performed examining 30-minute exposures to combinations of four gases - $\mathrm{CO}, \mathrm{CO}_{2}, \mathrm{HCN}$, and reduced $\mathrm{O}_{2}$ (Table 3 ). The additivity and synergism observed previously were still applicable for the various three and four gas combinations.

Based on these results, equation (1) was derived to predict the combined toxicities of these four gases:

$$
\frac{\mathrm{m}[\mathrm{CO}]}{\left[\mathrm{CO}_{2}\right]-\mathrm{b}}+\frac{[\mathrm{HCN}]}{\mathrm{d}}+\frac{21-\left[\mathrm{O}_{2}\right]}{21-\mathrm{LC}_{50} \mathrm{O}_{2}} \approx 1
$$

where the values in brackets are the atmospheric concentrations of the gases in ppm $\left(\mathrm{CO}, \mathrm{CO}_{2}, \mathrm{HCN}\right)$ or percent $\left(\mathrm{O}_{2}\right) ; \mathrm{m}$ and $\mathrm{b}$ are the slope and $\mathrm{y}$ intercept of the $\mathrm{CO} / \mathrm{CO}_{2}$ interaction curve; and [HCN] and $\mathrm{d}$ are the atmospheric test and the lethal concentrations of $\mathrm{HCN}$, respectively. The value of $d$ will vary depending on whether it is the within-exposure deaths or the within plus postexposure deaths that are of concern. Based on all the data to date in which some percent of the animals died (not 0 or 100\%), the mean value of equation 1 is 1.1 with $95 \%$ confidence limits ranging from 0.9 to 1.3 . In other words, at the mean value of $1.1,50 \%$ of the exposed animals would be predicted to die; whereas, below 0.9 or above 1.3 , none or all of the animals would be expected to die from these exposures, respectively.

When the toxicity of mixtures of sublethal amounts of $\mathrm{CO}$ and $\mathrm{CO}_{2}$ was tested previously, the $\mathrm{CO}_{2}$ increased the uptake rate of $\mathrm{CO}$ and consequently, the formation of carboxyhemoglobin ( $\mathrm{COHb}$ ). In addition, the presence of $\mathrm{CO}_{2}$ also produced a degree of acidosis that was greater than that from $\mathrm{CO}$ or $\mathrm{CO}_{2}$ alone; the time of recovery from the acidosis was delayed when $\mathrm{CO}_{2}$ was present in addition to the $\mathrm{CO}$. When these experiments were repeated at lethal concentrations of $\mathrm{CO}(6200 \mathrm{ppm})$, similar effects were noted (i.e., the $\mathrm{COHb}$ increased and the blood $\mathrm{pH}$ decreased at a faster rate when $\mathrm{CO}_{2}$ was present than when absent). The deaths of all the animals in these experiments prevented comparison of the recovery rates from the acidosis. However, the mean time to death was faster when the rats were exposed to the $C O$ in the presence of $\mathrm{CO}_{2}(11.9 \pm 3.6 \mathrm{~min})$ than to $\mathrm{CO}$ alone $(17.9 \pm 5.5 \mathrm{~min})$.

This year's research has been very successful and has resulted in the development of a 4-Gas Model to predict the toxicity of combustion products for different times and variable concentrations of gases. This 4-Gas Model is based on the combined additive and synergistic toxicities of $\mathrm{CO}, \mathrm{CO}_{2}, \mathrm{HCN}$, and reduced $\mathrm{O}_{2}$. We have also been able to show that the synergistic effects of $\mathrm{CO}_{2}$ seen at sublethal levels of $\mathrm{CO}$ are applicable at higher (lethal) concentrations of $\mathrm{CO}$.

Progress this year has also been achieved in the development of methodologies to reveal previously unknown sources of fire toxicity. Computer software has 
been developed to simulate the degradation of materials as a function of temperature and to make use of infrared measurements to identify the components and measure the concentrations of these components in fire atmospheres. Current studies have revealed unsuspected mechanisms of thermal degradation and correlations between polymeric structure and thermally induced product and energy distributions.

Funding from Other Agencies/Institutions

In addition to CFR Priority Project funding, the above research was partially supported with funds from The Society of Plastics, Inc., The International Isocyanate Institute and The U.S. Army Medical Research Institute of Chemical Defense.

\section{Reports and Publications}

1. Babrauskas, V., Levin, B.C., and Gann, R.G., A new approach to fire toxicity data for hazard evaluation. Fire Journal 81:22-71 (1987). Also in ASTM Stand. News 14:28-33 (1986).

2. Levin, B.C., Paabo, M., Gurman, J.L., Harris, S.E., and Braun, E., Toxicological interactions between carbon monoxide and carbon dioxide. Toxicology 47:135-164 (1987).

3. Levin, B.C., Paabo, M., Gurman, J.L., and Harris, S.E., Effects of exposure to single or multiple combinations of the predominant toxic gases and low oxygen atmospheres produced in fires. Fundam. Appl. Toxicol. $9: 236-250$ (1987).

4. Levin, B.C., Gurman, J.L., Paabo, M., Baier, L., and Holt, T., Toxicological effects of different time exposures to the fire gases: carbon monoxide or hydrogen cyanide or to carbon monoxide combined with hydrogen cyanide or carbon dioxide. Proceedings of the Ninth Meeting of the U.S.-Japan Panel on Fire Research and Safety, Norwood, MA, May, 1987. NBSIR 88-3753, National Bureau of Standards, Gaithersburg, MD, pp. 368-384 (April, 1988).

5. Complex Mixture Committee, "Complex Mixtures: Methods for In Vivo Toxicity Testing", National Research Council, National Academy Press, ISBN 0-309-03778-6, 375p. (1988).

6. Levin, B.C., Paabo, M., Gurman, J.L., Clark, H.M., and Yoklavich, M.F., Further development of the N-Gas Model. Report to Sponsor, June, 1988.

7. Nyden, M. R. and Babrauskas, V., "Use of FTIR Spectroscopy for Multicomponent Quantitation in Combustion Toxicology, "Abstracts from the Combined Meetings of the Eastern Section: Combustion Institute and the National Bureau of Standards Center for Fire Research Annual Conference for Fire Research, Gaithersburg, MD, pp 107-1 - 107-4, (November, 1987).

8. Nyden, M. R., Forney, G. F., and Chittur, K., "Spectroscopic Analysis of Strongly Interacting Systems: Human Plasma Protein Mixtures," Applied Spectrosc., 42: 588 - 593, (1988).

\section{Related Grants}

1. Southwest Research Institute, PI - Gordon Hartzell, Analysis of Hazards to Life Safety in Fires.

2. University of Pittsburgh, PI - Yves Alarie, Toxicity of Plastic Combustion Products.

3. University of California, PI - Peter Griffiths, Expert System Approach for Spectra/Structure Correlation for Vapor-Phase Spectra. 
TABLE 1. PREDICTABILITY OF $\mathrm{N}$-GAS MODEL USING $\mathrm{CO}, \mathrm{CO}_{2}$, AND $\mathrm{O}_{2}$

\begin{tabular}{|c|c|c|c|}
\hline \multirow{2}{*}{$\begin{array}{c}\text { N-Gas Prediction } \\
\text { Value }\end{array}$} & $\because$ & Deaths & \multirow[t]{2}{*}{ Day $^{\mathrm{a}}$} \\
\hline & WI & $\mathrm{PE}$ & \\
\hline 0.84 & $0 / 6$ & $0 / 4$ & - \\
\hline 0.89 & $0 / 6$ & $0 / 4$ & - \\
\hline 0.93 & $0 / 6$ & $1 / 4$ & 0 \\
\hline 0.96 & $0 / 5$ & $0 / 4$ & - \\
\hline 1.01 & $3 / 6$ & $0 / 3$ & - \\
\hline 1.06 & $4 / 6$ & $1 / 2$ & 3 \\
\hline 1.07 & $3 / 6$ & $1 / 2$ & 0 \\
\hline 1.12 & $4 / 6$ & $0 / 5$ & - \\
\hline 1.22 & $5 / 6$ & $0 / 1$ & - \\
\hline
\end{tabular}

TABLE 2. PREDICTABILITY OF N-GAS MODEL USING $\mathrm{CO}, \mathrm{CO}_{2}$, AND $\mathrm{HCN}$

\begin{tabular}{cccc} 
N-Gas Prediction & \multicolumn{2}{c}{ Deaths } & Day $^{\mathrm{a}}$ \\
Value $^{\mathrm{b}}$ & WI & PE & \\
\hline & & & \\
0.95 & $0 / 6$ & $1 / 6$ & 1 \\
1.01 & $0 / 6$ & $0 / 6$ & - \\
1.03 & $0 / 6$ & $1 / 6$ & 0 \\
1.11 & $0 / 6$ & $2 / 6$ & 1,3
\end{tabular}

TABLE 3. PREDICTABILITY OF $\mathrm{N}$-GAS MODEL USING $\mathrm{CO}, \mathrm{CO}_{2}, \mathrm{HCN}$, AND REDUCED $\mathrm{O}_{2}$

\begin{tabular}{cccc} 
N-Gas Prediction & \multicolumn{2}{c}{ Deaths } & Day $^{\mathrm{a}}$ \\
Value $^{\mathrm{b}}$ & WI & PE & \\
\hline 0.77 & $0 / 6$ & $0 / 6$ & - \\
0.91 & $0 / 6$ & $0 / 6$ & - \\
1.06 & $1 / 6$ & $1 / 5$ & 1 \\
1.08 & $0 / 6$ & $0 / 6$ & - \\
1.22 & $4 / 6$ & $0 / 2$ & -
\end{tabular}

a: Day that post-exposure deaths occurred.

$\mathrm{b}$ : Based on equation ( 1 )

WI: Within Exposure Deaths

PE: Post-Exposure Deaths 


\section{CENTER FOR FIRE RESEARCH \\ NATIONAL BUREAU OF STANDARDS \\ FY 88}

Institution:

Grant No.:

Grant Title:

Principal Investigator:

Other Professional Personnel:
Southwest Research Institute

60NANB6D0635

Analysis of Hazards to Life Safety

In Fires: A Comprehensive Multi-

Dimensional Research Program--Part 3

Dr. Gordon E. Hartzell

Institute Scientist

Department of Fire Technology

Southwest Research Institute

6220 Culebra Road

San Antonio, Texas 78238

Telephone: 512/522-3038

Dr. Arthur F. Grand (Staff Scientist)

Dr. Harold L. Kaplan (Manager, Inhalation Toxicology)

Howard W. Stacy (Assistant Manager, Inhalation Toxicology)

Walter G. Switzer (Sr. Research Scientist)

Dr. Barbara C. Levin

NBS Scientific officer:

Technical Abstract:

In order for a fire hazard model to be valid, it is essential that results obtained in combustion toxicity studies on materials be predictive, with a reasonable degree of confidence, of the effects of smoke exposure to humans. Because of potentially significant differences between the mouse or rat and the primate, studies were undertaken to investigate the suitability of other rodent species or to develop methodology that would allow rodent data to be used to better predict toxic effects in humans. The specific objective of this work has been to investigate the suitability of the guinea pig as a model for predicting the toxic effects of fire gases, particularly those containing irritants, in man.

Two $\mathrm{LC}_{50}$ values were determined for $\mathrm{HCl}$ with guinea pigs. The 30-minute exposure (plus 14-days post exposure) value was $1350 \mathrm{ppm}$, with 95-percent confidence limits of 1000 to $1800 \mathrm{ppm}$. The 15-minute exposure (plus 14 days) value was $2900 \mathrm{ppm}$, with 95-percent confidence limits of 1960 to $5300 \mathrm{ppm}$. Both values are significantly lower than those for rats, showing that the guinea pig is considerably more sensitive to $\mathrm{HCl}$ in terms of lethal toxic potency. Particularly striking was the incidence of within-exposure and same-day lethality for the guinea pig. This has never been observed with rats over the range of $\mathrm{HCl}$ concentrations used for $\mathrm{LC}_{50}$ determinations. Further- 
more, the effect appears more related to the length of the exposure, rather than to the dose. A possible explanation may lie in the marked tendency for bronchioconstriction by the guinea pig. Severe bronchioconstriction could contribute to a persistent hypoxic condition, leading to death either during or shortly after exposure.

Such bronchioconstriction does not necessarily mean, however, that the guinea pig is a better animal model for man. The guinea pig may actually be too sensitive. The comparison of lethal toxic potencies shown in Table I for rodents, including mice, rats and guinea pigs, with estimated data for baboons $[1,2]$, would seem to indicate that the rat (and perhaps even the mouse) is a reasonable model for primates and that the guinea pig is not.

TABLE I

LETHAL TOXIC POTENCIES OF HYDROGEN CHLORIDE

$\left(\mathrm{LC}_{50}\right.$ VALUES IN PPM)

\begin{tabular}{crccc}
$\begin{array}{c}\text { Exposure Time } \\
(\text { min) }\end{array}$ & Rats $^{\mathrm{a}}$ & Mice & $\begin{array}{c}\text { Animal Model } \\
\text { Guinea Pigs }\end{array}$ & Baboons \\
\hline 5 & 15,900 & $13,745^{\mathrm{b}}$ & & 17,000 \\
10 & 8,370 & $10,138^{\mathrm{c}}$ & & \\
15 & 6,920 & & 2,900 & 10,000 \\
22.5 & 5,920 & & & 5,000 \\
30 & 3,800 & $2,644^{\mathrm{b}}$ & 1,350 &
\end{tabular}

a. 14-days post exposure [2]

b. 7-days post exposure [3]

c. 3-hours post exposure [4]

d. 14-days post exposure (this study)

e. These are not $L_{50}$ values, but estimates based on conditions of animals after exposure. The 5-minute exposure resulted in post-exposure deaths [1]. The 15- and 30-minute exposures yielded subjects that survived indefinitely [2].

In preparation for further studies using the guinea pig, the 30-minute $\mathrm{LC}_{50}$ for carbon monoxide was determined to be $17,500 \mathrm{ppm}$ ( 95 -percent confidence limits of 14,900 to $21,400 \mathrm{ppm}$ ). This value is about three times that for the rat, showing the guinea pig to be remarkably insensitive to C0. Carboxyhemoglobin loading studies did show the guinea pig to load $\mathrm{CO}$ somewhat more slowly than the rat, but most surprising was the guinea pig's tolerance to $\mathrm{CO}$ concentrations that would easily cause lethality in rats.

A further demonstration of the guinea pig's insensitivity to CO was seen in studies exposing these animals to mixtures of $\mathrm{CO}$ and $\mathrm{HCl}$. These two toxicants are normally considered additive in their effects on rats [5]. However, with guinea pigs, the 30-minute (14-days post exposure) $\mathrm{LC}_{50}$ of $\mathrm{HCl}$, when in the presence of both $0.3 \mathrm{LC}_{50}$ and $0.5 \mathrm{LC}_{50}$ of $\mathrm{C} 0$, was essentially the same as for $\mathrm{HCl}$ alone. Reasonable evidence of additivity was obtained only at a combination of $0.76 \mathrm{LC}_{50}$ of $\mathrm{CO}$ and $0.37 \mathrm{LC}_{50}$ of $\mathrm{HCl}$. It would seem that the 
guinea pig is so insensitive to the $C 0$ that additivity is not observed until the $\mathrm{CO}$ concentration is quite high and well above that which would kill rats. Since it has been fairly well established that the rat is a reasonable model for $\mathrm{CO}$ intoxication of both baboons and humans [6], it must be concluded that the guinea pig is actually a very poor model. It would certainly appear to be inappropriate to use an animal model for combustion toxicity studies that is so insensitive to the most common fire gas toxicant.

Whether or not the guinea pig's greater sensitivity to $\mathrm{HCl}$ is meaningful in terms of human exposure is uncertain. The studies reported here address only lethal toxic potency and may or may not be relevant to sublethal effects. However, there seems to be mounting evidence that the rat is a reasonable model for both asphyxiants and irritants. There is little evidence that it is not.

\section{References:}

1. Kaplan, H.L., A.F. Grand, W.G. Switzer, D.S. Mitchell, W.R. Rogers and G.E. Hartzell, "Effects of Combustion Gases on Escape Performance of the Baboon and the Rat," J. Fire Sciences, Vol. 3, No. 4, pp. 228-244 (July/ August 1985).

2. Hartzell, G.E., S.C. Packham, A.F. Grand and W.G. Switzer, "Modeling of Toxicological Effects of Fire Gases: III. Quantification of PostExposure Lethality of Rats from Exposures to HCl Atmospheres," J. Fire Sciences, Vol. 3, No. 3, pp. 195-207 (May/June 1985).

3. Darmer, K.I., E.R. Kinkead and L.C. DiPasquale, "Acute Toxicity in Rats and Mice Exposed to Hydrogen Chloride Gas and Aerosols," Am. Ind. Hyg. Assoc. J., 35:623-631 (1974)

4. Alarie, Y., Proceedings of the Inhalation Toxicology and Technology Symposium, Kalamazoo, Michigan, October 23-24, 1980, B.K. Leong, Ed., Ann Arbor Science Publishers, Incorporated, pp. 207-238 (1980).

5. Hartzell, G.E., A.F. Grand and W.G. Switzer, "Modeling of Toxicological Effects of Fire Gases: VI. Further Studies on the Toxicity of Smoke Containing Hydrogen Chloride," Vol. 5, No. 6, pp. 368-391 (November/ December 1987).

6. Kaplan, H.L. and G.E. Hartzell, "Modeling ot Toxicological Effects of Fire Gases: I. Incapacitating Effects of Narcotic Fire Gases," J. Fire Sciences, Vol. 2, No. 4, pp. 286-305 (July/August 1984).

\section{Reports and Papers:}

1. Hartzell, G.E., A.F. Grand, and W.G. Switzer, "Studies on the Toxicity of Smoke Containing Hydrogen Chloride," Second International Symposium on Fire Safety Science, Tokyo, Japan, June 13-17, 1988. 
CENTER FOR THE FIRE RESEARCH

NATIONAL BUREAU OF STANDARDS

FY 88

Institution: Graduate School of Public Health, University of Pittsburgh

Grant No.: 60NANB8D0813

Grant Title: Toxicity of Plastic Combustion Products

Principle Investigator: Yves Alarie, Ph.D.

University of Pittsburgh

Pittsburgh, PA 15261

Telephone: (412) 624-3047

Other Professional Personnel: $\quad$ D. Malek, Ph.D.

F. Esposito, Ph.D.

NBS Scientific Officer: B.C. Levin, Ph.D.

Technical Abstract

An animal model has been developed to stimulate moderate exercise as may occur in individuals trying to escape a fire. The laboratory animal use is the guinea pig because the effect of asphyxiants and irritants, both always present in fire smoke, can be easily recognized in this species. Guinea pigs will also run well in an ergometer with no need for stimulating them to run. An ergometer was built to permit training each animal so that they will run at a constant speed and for a period of at least 45 minutes. The running speed was selected so that the oxygen uptake will be about two to three times the resting oxygen uptake. This simulate a level of activity in humans which would be described as "fast walk". The ergometer was built so that when enclosed it can function as a whole body plethysmograph to measure tidal volume and respiratory

frequency continuousiy as well as oxygen uptake and carbon dioxide output. The whole body plethysmograph/ergometer system is continuously ventilated and therefore exposure to single toxicants such as carbon monoxide, hydrogen cyanide etc as well as smoke from burning polymers can be introduced at any time to study their effects on the performance of the animal.

Experiments have been completed with this escape performance model using carbon monoxide as the first toxicant. Comparisons were made with the effect of the same toxicant in sedentary animals. The results have now been analyzed and furthermore comparisons made with predictions of human performance under the same toxicant.. The results in guinea pigs indicate that the effect of carbon monoxide in running animals occurred at about half the concentration and in about half the time for this toxicant to affect sedentary animals. When extrapolating these results to humans, using literature data on the incapacitating capacity of carbon monoxide in humans at moderate work, the data obtained in guinea pigs are in very close agreement for distance traveled before incapacitation. Therefore, if carbon monoxide is the principal toxicant in fire smoke these data can 
be used with confidence in computer modeling to predict escape capabilities. In fact, fire models including levels of carbon monoxide generation can now use levels of carbon monoxide generated to predict how long humans can function or travel before being incapacitated by this toxicant.

Experiments have also been completed using hydrogen chloride as the toxicant to test how this model can be used with irritants. With hydrogen chloride the difference between running animals and sedentary animals is much greater than for carbon monoxide. In sedentary animals a concentration of hydrogen chloride of 1,000 to $1,200 \mathrm{ppm}$ for 30 minutes produced no incapacitation or death. In contrast a concentration of hydrogen chloride of 500 to $600 \mathrm{ppm}$ incapacitated running animals and was lethal within 3 minutes. The lowest no effect concentration of hydrogen chloride in running animals is around $150 \mathrm{ppm}$. Extrapolating these results to humans is not as easy as for carbon monoxide since very few experiments have been conducted in humans with hydrogen chloride. The data in humans indicate that 70-100 ppm is the highest level that can be tolerated for a few minutes because of intense irritation and chest pains. Since running guinea pigs are not incapacitated for an exposure period of 30 minutes at $150 \mathrm{ppm}$ some correction factor will have to be developed to take into account the fact that guinea pigs are less sensitive to this irritant than humans are. Nevertheless this escape performance model is the first incapacitation, model described in the literature which can assess the effect of both asphyxiants and irritants. Also the incapacitation effect occurs at a much lower concentration and in a much faster time for carbon monoxide than the lethal effect which differentiates this model from all previous models where incapacitation and death occurred at almost the same concentration and time.

In depth investigations were conducted on the possible synergistic effects of fire gases such as carbon monoxide, hydrogen cyanide, carbon dioxide as well as low oxygen. Experiments at NBS used rats while experiments at the University of Pittsburgh used mice. The results are similar although different protocols were used. It is now clear that carbon monoxide and hydrogen cyanide act in a simple additive manner. is also clear that carbon dioxide will affect the toxicity of carbon monoxide or hydrogen cyanide but its effect is small and if the concentration is less than $10 \%$ toxic fire models can ignore its effect. The effect of low oxygen in combination with carbon monoxide or hydrogen cyanide cannot be ignored as easily. When oxygen was above $16 \%$ there was no detectable influence on the toxicity of carbon monoxide or hydrogen cyanide. When oxygen was below $13 \%$ the toxicity of carbon monoxide did not change greatly but the toxicity of hydrogen cyanide changed considerably. Its effect was to change both the time of exposure to induce a lethal effect (by a factor of 3 ) and to reduce the lethal blood cyanide level. With hydrogen cyanide exposure, the lethal level of cyanide in blood was found to be around $1 \mathrm{mg} / 1$ iter. This was reduced by a factor of 3 in the presence of low oxygen. In contrast the lethal level of carboxyhemoglobin was just slightly reduced when comparing lethal levels obtained with exposure to carbon monoxide alone versus carbon monoxide with oxygen levels below $13 \%$. This finding will complicate fire hazard modeling for toxicity when cyanide is present in fire gases. Indeed, of all the combinations used, hydrogen cyanide and low oxygen 
present the greatest lethal threat, rather than the combination of carbon monoxide and hydrogen cyanide. Obviously if both carbon monoxide and hydrogen cyanide are present the additive effect is present. However if hydrogen cyanide and low oxygen are present this combination is both more potent and faster acting. This combination also present more difficulties for modeling since instead of being additive as in the case of carbon monoxide and hydrogen cyanide the combination of hydrogen cyanide and low oxygen is of no consequence if oxygen is above $16 \%$ but of great consequence if oxygen is below $13 \%$. This difference of $3 \%$ is rather small when using fire models for prediction of oxygen levels in fire smoke.

In order to develop small scale toxicity assessment of fire smoke the cone calorimeter developed at NBS is being investigated to potentially replace the furnace system used to burn polymers. Preliminary experiments conducted at NBS two years ago indicated that this approach could be used. The instrumentation was built at NBS and delivered last September to the University of Pittsburgh. Major problems have been encountered using this system. So far these problems have been of a mechanical nature rather than fundamental. This is to be expected. The design of the cone calorimeter at NBS has been centered on the measurement of heat release and the parameters, in some instances, are in conflict with using the apparatus for toxicological studies. The basic cone heater and mass loss measuring load cell will be retained but the hood, enclosure, flow measuring devices and exhaust system are being modified. These changes are necessary for reducing the residence time of the fire smoke generated and to provide fire smoke at a temperature which will be compatible with minimum heat stress on the animals. Also the enclosure system is being changed to continuously measure airflow coming within the enclosure containing the heating cone and oxygen level will be continuously monitored in all exposure chambers since we have found that depletion of oxygen can have a very significant effect when hydrogen cyanide is present in fire smoke. 
CENTER FOR FIRE RESEARCH
NATIONAL BUREAU OF STANDARDS

FY 88

Institution: The University of California, Riverside

Grant No.: 60NANB7D0736

Grant Title: Expert System Approach for Spectra/Structure Correlation for Vapor-Phase Spectra

Principal Investigator: Professor Peter R. Griffiths

Department of Chemistry

University of California, Riverside

Riverside, CA 92521

Other Professional Personnel:

Dr. Dongjin Pyo

Postdoctoral Research Associate

Mr. Hsi-Tung Lin

Graduate Research Assistant

NBS Scientific officer:

Dr. Vytenis Babrauskas

Technical Abstract:

Introduction Rapid identification of the components of the atmosphere in the region of fires is not only important in combustion research but can also allow to have practical benefits. For example, the potential danger to firefighting personnel because of toxicity could be ascertained on site and, in certain cases, the cause of a fire may be found. Infrared spectrometry has the capability of allowing both major and minor components in the atmosphere to be identified and quantified. However, the infrared spectra of compounds in the vapor phase are not readily interpreted. Although Nyquist has described several correlations between vapor phase infrared spectra and the molecular structure of the absorbing molecule [1], these rules are not readily amenable to rapid computer interpretation. Thus they cannot yet be applied to the identification and quantification of molecules in the atmosphere near an actual fire. Even in laboratory studies of combustion processes, where spectroscopic data can be analyzed after the experiment is complete, the gaseous components would still be difficult to identify simply by measuring the infrared spectrum of the atmosphere and applying vapor-phase infrared spectra/structure correlation rules as they are presently understood. In this project, we are attempting to obtain a better understanding of the correlation between the structure of molecules and their vapor-phase infrared spectra through the use of contemporary "expert system" software.

Spectral Database The most common application for which vapor-phase reference spectra are used is for the identification of components of mixtures separated by gas chromatography (GC) using on-line Fourier transform infrared (FT-IR) spectrometry. In GC/FT-IR, spectra are usually measured at relatively low resolution $\left(8 \mathrm{~cm}^{-1}\right)$ and computed with a sampling interval of $4 \mathrm{~cm}^{-1}$. To reduce the time required for searching GC/FT-IR data, the spectra are then 
"deresolved", 1.e. the sampling interval is reduced to $8 \mathrm{~cm}^{-1}$. At least two databases of deresolved reference spectra are commercially available. However, much significant information is lost from vapor-phase infrared spectra when they are deresolved. If expert system software is to be used for generating useful spectra/structure correlations, there is good reason to belleve that the resolution of the spectra should be no worse than $4 \mathrm{~cm}^{-1}$.

A large database of vapor-phase infrared reference spectra has been measured by the personnel of Sadtler Research Laboratories, Division of Bio-Rad Laboratories (Philadelphia, PA). The original spectra in this database were measured at $4 \mathrm{~cm}^{-1}$ resolution with data points at $2 \mathrm{~cm}^{-1}$ intervals, so that these spectra appear sultable for our purpose. The original spectra in this database are not usually made avallable to the public because of their proprietary nature. Before this project was started, however, the Principal Investigator was told that he would be able to lease the entire collection of original spectra from Sadtler Research if this project was funded. Unfortunately, after the project was funded, Sadtler required that a very restrictive licensing agreement be signed. It has taken over ten months from the start of this grant for the University of California, Riverside (UCR) and Sadtler Research Laboratories to agree on the specific terms of the license. Even then, the data are to be sent to UCR in an encrypted form to ensure their protection. As yet, the database has still not been recelved at UCR, and a one-year extension of this grant has been requested to enable the proposed work to be completed.

Band Parameter Calculation For the application of most expert system sof tware to find spectra/structure correlations, spectra must be changed from their usual format of (absorbance, wavenumber) pairs. We are developing techniques for accurately calculating the parameters, $1 . e$. the center wavenumber, peak absorbance and full-width at half-height (FWHH), of all bands in the spectrum, including those bands that are partially or completely unresolved from a neighboring spectral feature. The technique that is being used involves first narrowing each band by Fourier self-deconvolution (FSD) and then fitting the deconvolved spectrum (in which all significant bands have been resolved) by a series of Lorentzian-Gaussian bands.

An algorithm has been developed to find the optimum parameters for vaporphase spectra. The widths of each band in a given spectrum are progressively reduced by an amount, $\gamma^{\prime}$. The series of spectra calculated in this manner is curve fit, and the sum of the squares of the difference (SSD) between each synthetic spectrum and the corresponding deconvolved spectrum is calculated. The conditions for calculating the most accurate band parameters can be found from plots of SSD against $\gamma^{\prime}$. The band parameters calculated in this way will ultimately be used as input to the expert system programs.

Expert System Programs A listing of available expert system programs for operation on an IBM-AT compatible personal computer was obtained. From this list, two programs were selected as being the most appropriate for our purpose. The first (Prolog, Arity Corp., Concord, MA) proved to be less useful than the second (lst CLASS, Programs in Motion, Wayland, MA, and the latter program will be used for much of the future work on this project. In addition to the commercial software, programs have been written at UCR for testing some simple concepts; the early results obtained using these programs are described below. 
Wavenumber-Based Spectra/Structure Correlations

In infrared spectra, it is usually impossible to determine the structure of any compound on the basis of absorption in only one region. To determine the structural units present in a compound by the use of group frequencies, it is therefore necessary to examine several spectral regions. Finding the group frequencies for each functional group is the most important step for structural elucidation using Infrared spectra. For our early studles, band parameters for the vapor-phase spectra of several carboxylic acids were calculated in the manner described above to give "peak table spectra". A simple routine was written to show the feasibility of determining the characteristic regions for absorption by a certain functional group. In this approach, the peak table spectra are reformatted as binary points along the wavenumber axis, with all points one full width at half height on either side of the band center being represented as ones. All other points are represented as zeros. (For more specific spectral representation, a region for each band that is bounded by a fraction of the FWHH can obviously be selected). As an example, the high wavenumber region of the spectrum of butyric acid would be represented as follows:

\begin{tabular}{|c|c|c|}
\hline 1 & $\mid 2 \times$ FWHН $\mid$ & $\mid 2 \times$ FWHH $\mid$ \\
\hline $\begin{array}{l}4000 \mathrm{~cm}^{-1} \\
(\text { START })\end{array}$ & $\begin{array}{l}3602 \cdots 3557 \\
(0-H \text { Stretch })\end{array}$ & $\begin{array}{c}3017 \cdots \cdots 2929 \mathrm{~cm}^{-1} \\
(\mathrm{C}-\mathrm{H} \text { Stretch })\end{array}$ \\
\hline
\end{tabular}

Binary Representation of Butyric Acid Spectrum

By overlapping spectra that have been formatted in this way, characteristic regions for a given functional group can be selected by finding the regions common to that class of spectra, $1 . e$. , the regions where each spectrum contains ones. An occurrence value for every wavenumber can be calculated simply by adding the binary spectra, so that if an occurrence value equal to the number of spectra is computed in a given region, that region would be a candidate for the spectra/structure correlation table. Using the binary spectral data for eight carboxylic acids whose reference spectra were available in the EPA library of vapor-phase infrared spectra, occurrence values for every wavenumber were calculated by overlapping and summing these eight binary spectra. Results were obtained with threshold occurrence values of $100 \%$ (i.e., all of the acids absorbed in this region) and $88 \%$ (i.e., 7 of the 8 acids absorbed in this region). As would be expected, broader regions were calculated with a threshold occurrence value of $88 \%$ than with a threshold occurrence value of $100 \%$ and additional spectral regions, corresponding to the vibrational modes of functional groups not associated with the COOH moiety, were added. This approach is belleved to be quite promising, and will be investigated further when we receive the full Sadtler database.

Incorporation of Peak Intensity into the Rules In previous work, detailed absolute or relative peak intensity information has rarely been used in the interpretation of infrared spectra. In an attempt to get less equivocal structural information than can be found using only wavenumber-based spectra/structure correlations, we are starting to incorporate intensity information in the input to our expert system program for structural elucida- 
tion. To show the feasibility of using intensities for this purpose, peak table spectra of the same elght carboxylic acids used above were normalized to the most intense band in the spectrum (which in all cases was the $C=0$ stretching band). To characterize the band intensity in a given spectral reglon, the absorbances of all spectra were averaged between wavenumbers located between one FWHH elther side of the center wavenumber; the average relative peak intensity and standard deviation was then calculated in each region. The best correlation was found between the intensity of the $0-H$ stretching and $\mathrm{C}=0$ stretching bands of the $\mathrm{COOH}$ group. Since all these compounds were carboxylic acids, these preliminary results give rise to a certain amount of optimism that relative band intensities will prove to be more useful in structural elucidation than has been recognized previously. We will therefore attempt to incorporate information of this type in the expert system programs.

\section{Reference}

1. R. A. Nyquist, "The Interpretation of Vapor-Phase Infrared Spectra", Vol. 1, Sadtler Research, Philadelphia, PA, 1984. 


\section{CENTER FOR FIRE RESEARCH \\ PRIORITY PROJECT - 1988}

FIRE DATA FOR MATERIALS

\section{Professional Personnel}

Sanford Davis, Project Leader

Emil Braun, Physicist

\section{Project Objective}

To document the fire performance of materials, components, systems, and assemblies and to develop the protocols for obtaining, assessing, and evaluating input data for fire models and other predictive methods.

\section{$\underline{\text { Scope }}$}

To conceive, develop, and implement projects relating to the evaluation and adaptation of databases for use in hazard assessment models.

\section{Technical Accomplishments}

Two milestones were achieved this year: first, participation in the National Fire Research Strategy Conference Hazard Database Task Group and, second, the conceptual development of a second generation HAZARD database for CFR. The design requirements of this database are described in a draft papaer currently undergoing editorial review. The long-term goals of these two groups - Hazard Database Task Group and CFR - vis-a-vis a material fire property database, are different. These divergent goals will have to be addressed in the future and may necessitate the design of several different databases rather than a single database meeting the needs of both groups.

The long-term objectives of the Hazard Database Task Group are:

1) development of standard reporting forms for a large number of test methods;

2) making a large body of testing information available to the fire, design, and regulatory communities.

In contrast, the long-term objectives of CFR are to:

1) provide a collection of data needed for hazard assessment, as defined in HAZARD I;

2) reduce the information retrieval and transfer burden for users of HAZARD N.

The Hazard Database Task Group held two meetings during which a plan for action was developed. Six major issues were addressed. They are: 


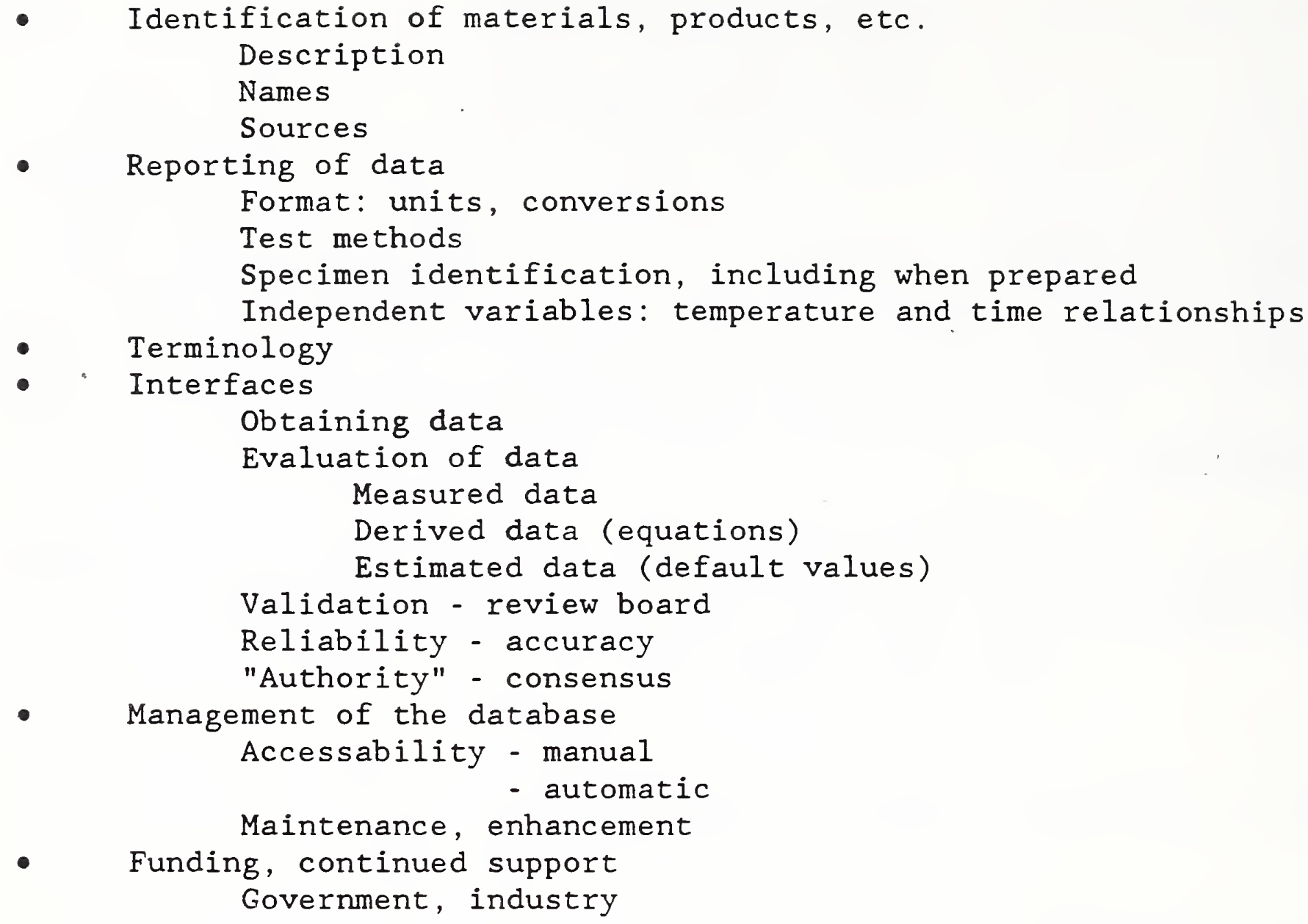

A paper describing the approach to the development of a National Fire Database has been written for publication in the model code group periodicals. The intent is to inform code and building officials of the need for input data for hazard assessment models. The National Fire Database would contain welldocumented data obtained from standard fire performance tests. Building codes specify fire performance requirements for materials of construction, and for some building contents, and governmental regulatory agencies have requirements for materials used in aircraft, rail vehicles, and sea-going vessels. The inclusion of fire performance data in a database would facilitate decision making for materials and product selection. The thrust of this paper is to describe the process and to propose a database management system for implementation of the process.

Consideration has been given to the presentation of the data in the database. An example of how computer-accessed data would be presented for a particular building material is shown here for purposes of discussion.

\section{Entry}

Class (or intended use)

Type of product

Source

Designation

Material

Form

Density, $\mathrm{kg} / \mathrm{m}^{3}$

Thickness, mm

\section{Example}

wall lining

wood

Weyerhaeuser

Versaboard

Douglas fir

plywood

640

13 
Property (1)

Laboratory

Test method

Test number (if any)

Date of test

Orientation

Ignition mode

Ignition enérgy, $\mathrm{kW} / \mathrm{m}^{2}$

Ignition time, sec

Heat of combustion (aver), $\mathrm{kJ} / \mathrm{kg}$

Co yield (aver), $\mathrm{kg} / \mathrm{kg}$

$\mathrm{CO}_{2}$ yield (aver), $\mathrm{kg} / \mathrm{kg}$

etc.

Extinction area (aver), $\mathrm{m}^{2} / \mathrm{kg}$

Time dependent data

$$
0
$$

10

20

30

..

100

110

...

. .

960

1020 rate of heat release

National Bureau of Standards

cone calorimeter/ASTM P190

1264

$04 / 16 / 91$

horizontal

piloted (spark)

25

26

12800

0.0008

0.0048

32.9

Heat release Mass loss CO etc.

Other properties (ignitability, flame spread, etc.) are included as an extension of this table. The user of the database calls up a product and automatically retrieves all the data input required by the hazard model. A specifier would have ready access to a product's fire characteristics to fulfill his needs for procurement purposes.

Current work is in progress to enhance the pilot database developed for HAZARDI in order to provide fire performance characteristcs for additional products and to add to the base of thermophysical properties of construction materials. As the next version of the hazard assessment model is being developed, it will be necessary to reformat the available data for direct transfer into the appropriate segment of the hazard analysis process. This will make running of the model more "user friendly" and facilitate access to the data. What data will be included, how they will be evaluated, what format for the data is required, how they will be input, and how they will be presented are currently under consideration.

Reports and Publications

"National Database for Fire Hazard Analysis", S. Davis and E. Braun, to be published. 


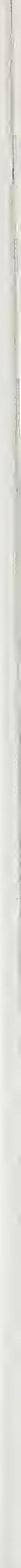


CENTER FOR FIRE RESEARCH

PRIORITY PROJECT - 1988

CONE CALORIMETER DEVELOPMENT

\section{Professional Personnel}

Vytenis Babrauskas, Project Leader

Kay Villa, Textile Technologist

Marc Janssens, Research Associate, National Forest Products Association

\section{Project Objective}

To further develop the Cone Calorimeter as a useful engineering tool for characterizing combustion properties of products and materials.

Scope

1. To complete the ASTM and ISO round robins

2. To achieve full operation of both NBS Cone Calorimeter units, including FTIR hookup

3. To begin the conversion of Cone Calorimeter data files into a proper database, suitable for modeling purposes and for inter-laboratory data exchange.

\section{Technical Accomplishments}

Because of slightly different interests of the laboratories concerned, the round robins sponsored by ASTM and by ISO have evolved as closely related, but not identical, programs. The ASTM round robin uses 6 materials: ABS, particle board, PMMA, polyethylene, PVC, and rigid polyurethane foam (aircraft material). The test conditions comprise 25 and $50 \mathrm{~kW} / \mathrm{m}^{2}$ irradiances, in both horizontal and vertical orientations (excluding those specimens which are incompatible with a given orientation). These specimens will be tested both by the 6 U.S. labs under ASTM's auspices and by the 7 European and Japanese labs under ISO. The ISO labs (+ NBS) will also be conducting tests on a series of materials of interest to the ISO community. These are: plywood, FR plywood, rigid polyurethane foam (for buildings), rigid polystyrene foam, and gypsum wallboard. Testing will be done at the same irradiances and orientations as for the ASTM materials.

Prior to the actual start of testing, two calibration steps were taken. All the heat flux gauges used in the different laboratories were calibrated against an NBS standard gauge. (2) A supply of calibration PMMA was issued to the participating laboratories. Only upon verifying by NBS that credible results were being obtained with the PMMA, would the actual round robin work begin. 
A User's Guide for the Cone Calorimeter has been prepared and issued. This document (118 pp.) is, by far, the most detailed operating guide ever prepared for a fire test method. The Guide includes an extensive troubleshooting section; further experience from users will provide an expansion of this section in the next edition.

Some progress was made on the augmentation of Cone Calorimeter physical facilities at NBS. Certain needed work (e.g., the installation of the FTIR sampling system) was not started due to insufficient funds. During the year, however, significant progress was made on the installation of the second, controlled-atmospheres Cone Calorimeter unit. This installation is now complete, and data will be taken with the system shortly.

About a year ago, it was realized that the value of material fire properties, such as obtained from the Cone Calorimeter and other modern fire test devices, is much diminished if the data cannot be conveniently exchanged among laboratories, databased, searched, graphically manipulated, and cross-compared. Due to incompatible hardware and data format standards (and the unavailability of specifically tailored, yet user-friendly software), data exchange and comparison even within one laboratory has been difficult. So has the import of materials property data into computer fire models. To resolve this situation, it was decided to formulate standards for a unified Fire Data Management System. This would have the capability to access measured data from the Cone Calorimeter, the Furniture Calorimeter, and other current-day test methods. It would, equally well, take in simulation data, as generated from room fire models or calculations. In turn, it would have the capability to output the needed material fire property data into computer models. Once a standard data format is available, interchange of data among laboratories would become easy. So far, this work is being started up in cooperation with the Fire Research Station (England) and the Statens Provningsanstalt (Sweden). It is expected that other fire laboratories will be joining the effort shortly.

Progress on the software unification has been the following so far. A set of computer hardware to run on was selected. A programming language and programming utilities were selected. Preliminary data formats, for both vector and scalar data, were established. An over-all work plan was written, and certain portions expanded into pseudocode. Routines for physical-data acquisition were implemented.

Also, under the Research Associate program with NFoPA, an exhaustive survey of wood materials was made to collect the rate of heat release data taken not only from the Cone Calorimeter, but also from older apparatuses and in large-scale measurements. Data analysis is underway.

Reports and Publications

Twilley, W.H., and Babrauskas, V., User's Guide for the Cone Calorimeter. [U.S.] Nat. Bur. Stand. (1988).

Babrauskas, V., and Wickström, U.G., The Rational Development of Bench-Scale Fire Tests for Full-Scale Fire Prediction, to appear in the proceeding of Fire Safety Science, Second International Symposium (1988). 


\section{Professional Personnel}

Richard W. Bukowski

Walter W. Jones"

\section{Project Objective}

Develop methods for the quantitative assessment of fire hazard and support the implementation of these methods by the fire protection community.

\section{$\underline{\text { Scope }}$}

The project is intended to produce a fundamental capability to analyze the hazards associated with a specified fire scenario(s). This capability will be provided in the form of a fully-supported software package for MS-DOS personal computers. While initially limited to residential-style occupancies, the software design will be such that it can be used across a broad range of applications from fire safety education to fire reconstruction.

\section{Technical Accomplishments}

\section{HAZARD I Beta test}

The main thrust of the project this past year has been the user evaluation of the prototype version of the Hazard Assessment Method - HAZARD I. This consisted of a three volume (1000 page) report and a set of $51 / 4$ in. disks containing the software necessary to conduct hazard analyses of products used in residential occupancies. All of the software provided will operate on any IBM PC (XT or AT) or compatible computer. Figure 1 shows the information flow among the programs, which include a scenario development guidance utility; an interactive program for inputing data to the fire model; a data base program and files of thermophysical, thermochemical, and reference toxicity data; the FAST model for multicompartment energy and mass transport; a graphics utility for plotting data; a detector/sprinkler activation model; an evacuation model which includes human decision/behavior; and a tenability model which evaluates the impact of the predicted exposure of the occupants in terms

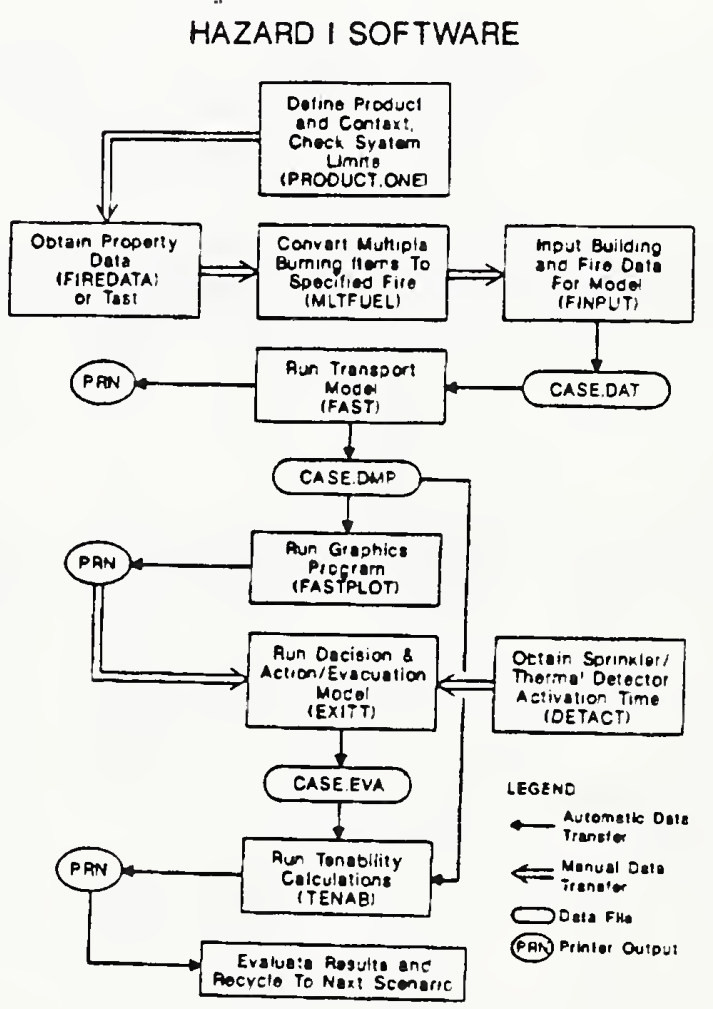

Figure 1 
of incapacitation or lethality from temperature or toxic gases or incapacitation by second degree burns from radiant flux exposure.

The prototype software was evaluated by a group of 93 international participants representing all aspects of the fire community. After installing the software on their own computer, they used the supporting documentation to learn the operation of the system and examine its capabilities to address problems within their own area of interest. While each committed to attempt and report on an analysis of their own choosing, only one such documented case was submitted. Many others reported conducting a case study, only to find that they had insufficient time to document it, or that the work done was proprietary in nature and could not be shared at this time.

The suggestions received from the evaluators, along with our own planned improvements were incorporated into the general release version of the software to be made available in December. Significant improvements were made, particularly in the user interface. The experience gained through the user evaluation was crucial to the production of a software package which promises to revolutionize the conduct of fire protection engineering.

Even though it is a prototype in limited release, HAZARD $I$ is beginning to find acceptance as a tool in the practice of fire protection engineering. A major consulting firm reports success in obtaining waivers from code authorities based on analyses conducted with the package. The International Conference of Building officials (ICBO) has requested training of their entire headquarters staff in the use of HAZARD I to evaluate code changes and new materials or products. And FAST was qualified as a "valid scientific tool" in a criminal (arson) trial in state court (Minnesota)

\section{Technical Improvements to FAST}

The FAST model forms the core of the hazard assessment capability, and the expansion of its capabilities are crucial to the growth of HAZARD. To this end, several technical improvements have been implemented in version 18 of FAST. These include a new, and more complete combustion chemistry scheme than has been included in other fire models. As shown in the diagram of figure 2, this scheme includes entrainment by and burning of the fire plume in both the lower $\left(e_{1}\right)$ and upper $\left(e_{2}\right)$ layers. The energy release is limited by the oxygen entrained into the plume,

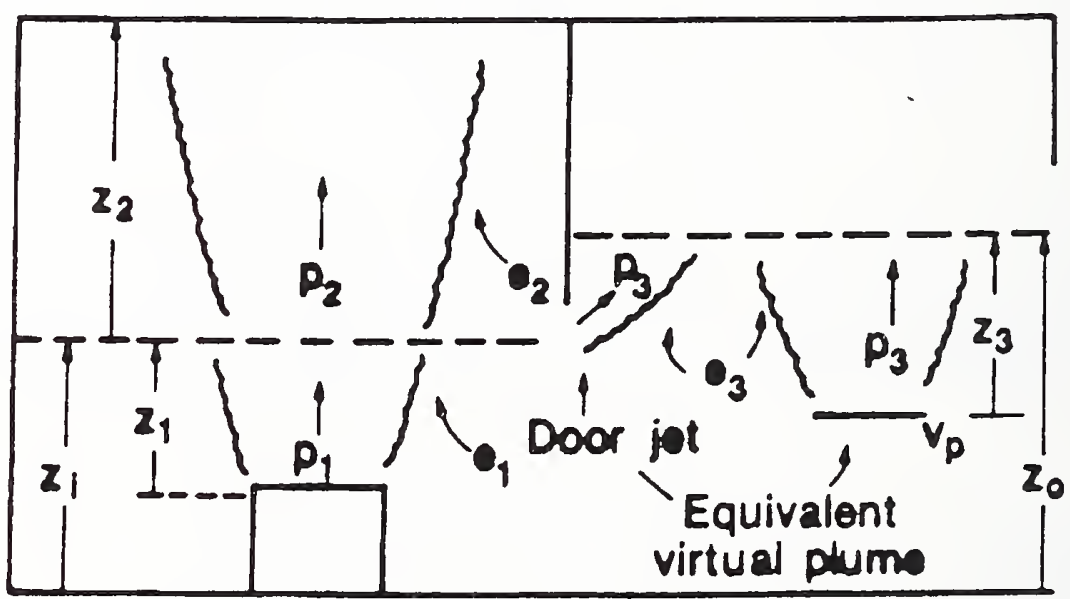

Figure 2 and its concentration in the layers. Fuel which cannot burn due to insufficient oxygen in the room of origin is tracked through other building spaces and burns when it mixes with sufficient 
oxygen (e.g., $e_{3}$ ). For the physical arrangement shown in figure 2, figure 3 shows how the energy from a $100 \mathrm{~kW}$ fire in compartment 1 would be distributed among the two layers in the compartment of origin (dashed, lines), the second compartment, and the vent to the exterior.

Another new feature is the ability to open and close, both interior and exterior openings at any time. This provides a unique capability to study the impact of changing ventilation created by evacuation, fire fighting operations, or even failure

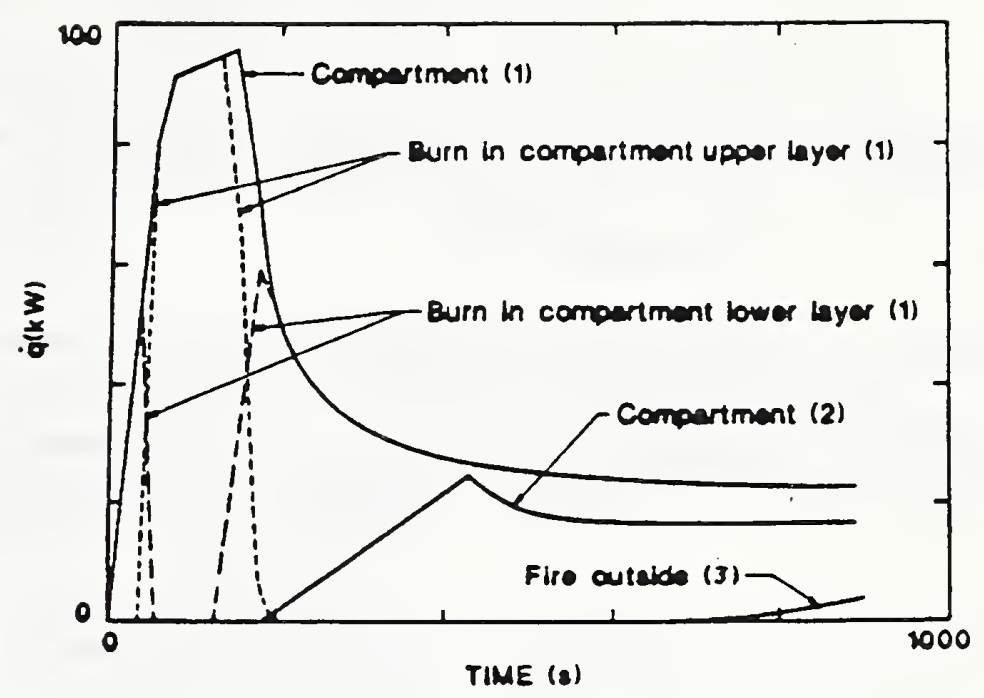
of a partition. Coupled with the vitiated combustion routine, this moves the analytical capability of FAST squarely into the realm of the fully-developed fire. Still lacking is a more detailed treatment of combustion, including such aspects as radiation to combustible items, enhancing their burning rate or driving the ignition and flame spread processes. Here, much progress has been made in the University of Dayton model which is directly linked to FAST.

\section{Presentations and Publications}

1. Jones, W. W., FAST and the Analysis of Fire and Smoke Hazard, Invited presentation at the Annual Meeting of the Sheet Metal and Air Conditioning Contractors Assn., Las Vegas, NV. (1988).

2. Jones, W. W., Refinement and Experimental Verification of a Model for Fire Growth and Smoke Transport, Second International Symposium on Fire Safety Science, Tokyo, Japan (1988).

\section{Related Grants}

"Mathematical Modeling of Furniture Fires", Mark A. Dietenberger, University of Dayton Research Institute. 


\section{CENTER FOR FIRE RESEARCH \\ NATIONAL BUREAU OF STANDARDS \\ FY88}

Institution: University of Dayton Research Institute

Grant No.:- 60NANB5D0556

Grant title: Mathematical Modeling of Furniture Fires

Principal Investigator:

Mark A. Dietenberger

Research Physicist

University of Dayton Research Institute

300 College Park - JPC/232

Dayton, Ohio 45469

(513) $229-4417$

Other Personnel:

Joyce Smith, Research Programmer

NBS Scientific Officer:

Dr. Vytenis Babrauskas

\section{Technical Abstract:}

Introduction HEMFAST models time-dependent, quasi-three-dimensional predictions of furniture fire growth and burnout in a room as well as of the spread of nontoxic and toxic gases and smoke to other rooms. The threedimensional aspect of the fire model includes a mockup construction with connecting panels, a flame volume with pyrolyzing polygonal base and sides, a radiation heat transfer between grey facets of objects and of the flame, and the three zones of gaseous flows. The time-dependent aspect of the fire model includes the flame spreading in any direction on the mockup, the scaled burning history of burning facets on mockup panels which includes mass and heat release as well as soot and toxic gas production, the temperature rises of the mockup facets, walls, and gas layers, and the growth of the upper gas layer. The predictive aspects of the fire model demonstrate recent physics models (with appropriate numerics of material heating, flame spread, material pyrolysis, flaming combustions, fire plumes, and gas flows. Finally, almost any furniture material can eventually be simulated because the model relies on effective scaling of the cone calorimeter data and of the flame spreading data. All these features taken together make HEMFAST an unique fire model. The highest priorities of the current research were (1) to simplify and make clear the interfaces between FAST and the Furniture Fire Model (FFM) and (2) to validate the second version of HEMFAST with full-scale fire tests.

HEMFAST Code Interfaces Two phases were taken to improve the interfaces between FAST and FFM. The first was to develop a main routine to divide the FAST code into the first part which reads in the FAST database and the second part which executes the fire growth algorithms. The subroutines for reading and processing the FFM database were placed between the two parts of the divided FAST code. This is shown in Figure 1. A user interactive menu was constructed in the main routine to manage the combined database. In the second phase, the interfacing subroutines were inserted into three FAST subroutines to manage the execution of FFM modules in conjunction with FAST fire simulation. One subroutine is used to synchronize the furniture fire 
growth computations as a function of time with the corresponding FAST time integrations. One other subroutine is used to convert FFM predictions to inputs needed by.-FAST and to bypass several FAST subroutines. The third interfacing subroutine is for printing out FFM predictions. These four interfacing routines were absent in the first version of HEMFAST installed at NBS. The absence of these routines gave the false impression that the FAST program was being profoundly modified.

These interfacing routines and a substantial amount of internal documentation has been incorporated with the second version of HEMFAST. Thus there is a basis for incorporating the FFM code to newer versions of FAST, or to incorporate a recent version of FAST (or even the HARVARD CFC) to any newer versions of the FFM.

Validation Process To validate the second version of HEMFAST with full-scale fire tests, it was necessary to revise the numerical techniques of time integration both within FFM and in its coordination with FAST's time integration. It was also necessary to incorporate our latest results on scaling some recent cone calorimeter database into HEMFAST by revising the bench scale data processors for the second version.

We acquired the furniture/room fire tèsts database from Dr. Vito Babrauskas, Mr. Richard peacock, and Dr. James Quintiere in August 1987. This database has been examined to select measured variables for comparisons with the predictions of the second version of HEMFAST. In particular, mockup burning rates, burning regions, and air temperatures as a function of time will be compared between model and experiment. The comparisons will be published in an upcoming report of the current grant and the HEMFAST (second version) code and its input/output wlll be presented to NBS. There should be no difficulty in implementing the code on NBS-CFR computers because of our experience with the first version of HEMFAST.

Conclusion Much work still remains even after the successful model validations, because a robust, somewhat user friendly, flexible, and documented fire model is still needed to gain full acceptability from the fire safety community. Thus the remaining work in the current grant period will be to start on the third version of HEMFAST.

\section{References}

r1. M. A. Dietenberger, "Improved Furniture Fire Model Within

'FAST'; HEMFAST-2", NBS-GCR-88-545, March 1988. 


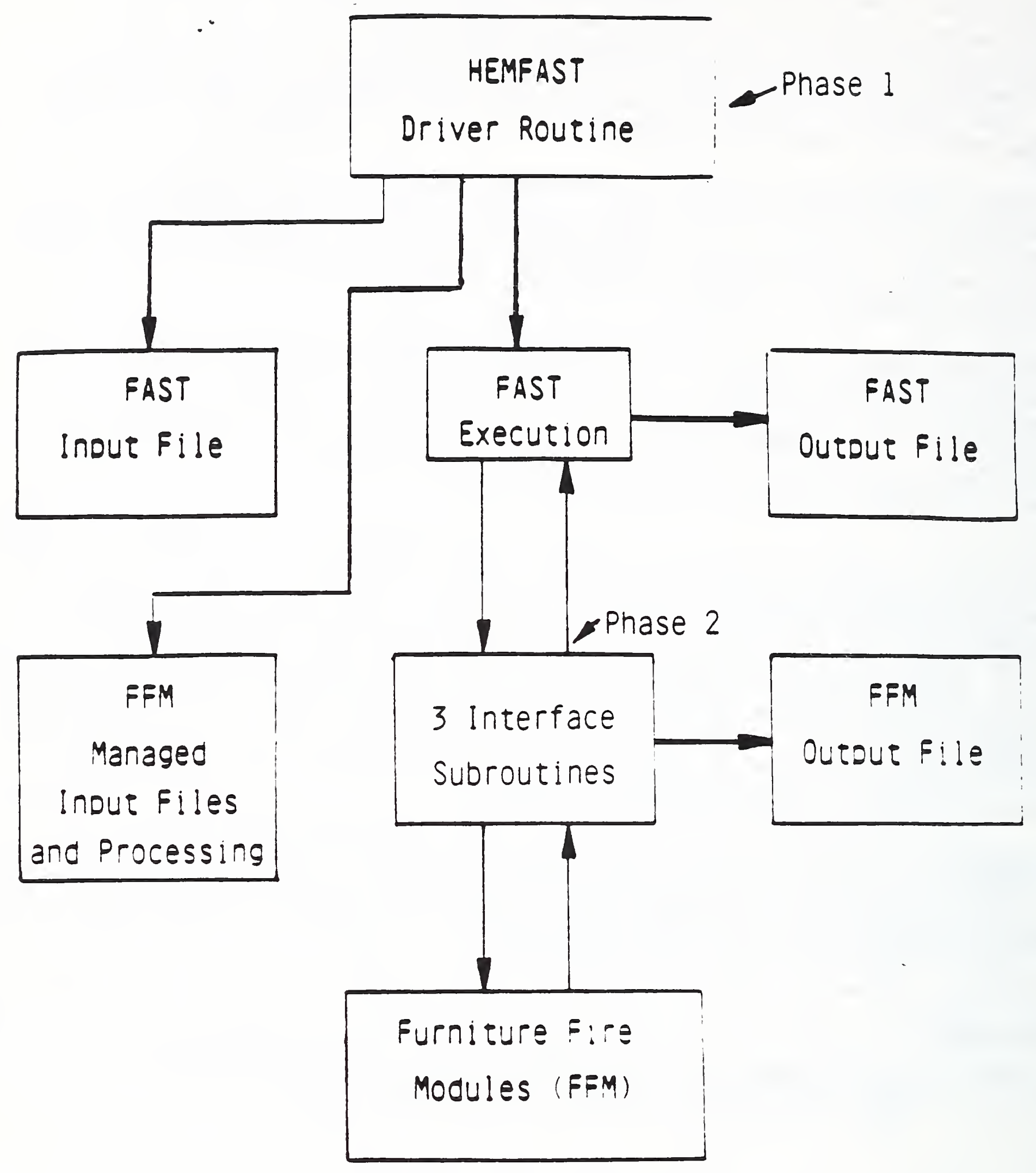

Figure 1. Revised Code Structure of Second Version of HEMFAST. 
PART I. Basic Research and Tools for "Engineered" Fire Safety (In-House Projects and Associated Grants Funded by NIST)

E. FIRE PROTECTION TECHNOLOGY 


\section{ENGINEERING ANALYSIS SYSTEM \& FIRE RECONSTRUCTION}

\section{Professional Personnel}

Harold E. Nelson, Project Leader

Robert Levine, Senior Scientist

D. Stroup, Fire Protection Engineer, Fire Suppression Research

C. Arnold, Computer Programer

\section{Project Objective}

1. The development of separated and assembled fire protection analytical (computerized) tools and the transfer of these tools to practicing professionals.

2. The use of these tools and other resources to recreate and otherwise analyze one or more significant fires. To initiate verification of the results, to evaluate the tools, and to assist decision makers in actions to prevent recurrence.

\section{Scope}

1. Assemble useful data, formulas, and simple models. Program these into a common format with an accessible point of entry (menu, etc.) Develop this into a generalized engineering approach suitable for compartmented buildings. (This portion of the project is closely allied with aspects of the GSA sponsored Engineered Fire Protection Assessment System project.)

2. Demonstrate procedures for fire reconstruction investigations. Prepare a kit of needed materials. Conduct a reconstruction investigation of one or more fires of significance. Analyze the fire, determine and initiate research or testing needs required for a satisfactory reconstruction, prepare the appropriate report.

\section{Technical Accomplishments}

1. Engineering Analysis System.

Several internal improvements expanding the capabilities and usefulness of FIREFORM have been added to that collection and issued as modified versions of FIREFORM. Major work continues to produce a greatly enhanced version (FIREFORM II) and to collect the major elements into a hazard analysis procedure (FPETOOL.)

2. Fire Reconstruction.

Two major fire investigations have been undertaken. 
a. Sharon, PA fire of Sept. 1987. This two story dwelling fire (3 fatalities) has been analyzed and on Sept. 22, 1988 a full scale simulation test was conducted. The test demonstrated the high $\mathrm{CO}$, low $\mathrm{O}_{2}$, low temperature lethal conditions suspected in our investigation and indicated by the analysis. While the analysis predicted the ultimate levels involved, the speed of development of lethal conditions was faster than expected.

b. First Interstate Bank Building, Los Angles, CA fire of May 4, 1988 . While the investigation has been completed, the analysis and preparation of the report are still in process.

\section{Funding from Other Agencies/Institutions}

In addition to CFR Priority Project funding the above activities were carried out with significant support from the General Services Administration (GSA)

\section{Reports and Publications}

Nelson, H. E., Concepts for Life Safety Analysis, Fire Safety Journal, Vol. 12, No. 3, 167-177, December 1987

Nelson, H. E., Engineering Analysis of the Early Stages of Fire Development-. The Fire at the Dupont Plaza Hotel and Casino--December 31, 1986, NBS Report NBSIR 87-3560.

Nelson, H. E., Engineering Fire Protection Design Assessment System. In NBS Report NBSIR 88-3753

Nelson, H. E., An Engineering Analysis of Fire Development in the Hospice of Southern Michigan, December 15, 1985, Presented at the 2nd Symposium of the International Association for Fire Safety Science, Tokyo, June 17, 1988

Nelson, H. E., Overview - Numerical Grading Systems, Transcript of Workshop/Conference on Analytical Methods of Fire Safety Design, Oct. 1416,1987, Building Research Board, National Academy of Sciences 
FIRE SUPPRESSION

\section{Professional Personnel}

David D. Evans, Head, Fire Suppression Research (Project Leader) William D. Walton, Fire Prevention Engineer James R. Lawson, General Physical Scientist Daniel Madrzykowski, Mechanical Engineer

\section{Project Objective}

Develop understanding of fire extinguishment processes and derive techniques to measure and predict the performance of fire protection and fire fighting systems.

\section{Scope}

This program is designed to assess the performance of fire extinguishing methods through physical measurement of fire phenomena during suppression. These measurements form the basis for generating the needed fire science and engineering knowledge to allow for prediction of fire suppression events. The predictive methods are developed to enhance CFR fire models.

\section{Technical Accomplishments}

\section{Wood Crib Fire Suppression}

Data from a series of water spray suppression tests was analyzed to produce two correlations; One for time to extinction and the other for energy release rate during suppression. These correlations will be the basis for the future fire suppression module to be added to the HAZARD I fire model.

The time to extinction was correlated using the method developed by Tamanini (Comb. Sci. Tech. 1976, Vol 14, p 17-23) for large normalized water application rates. At low normalized water application rates the correlated dimensionless time to extinction for Tamanini's small wood cribs $(1 / 2 " \mathrm{x} 5 \times 8$ $x$ 10) was found to be much greater than that measured for the large cribs $\left(1.5^{\prime \prime} \times 6 \times 16 \times 16,100 \mathrm{~kg}\right.$ mass and energy release rates of $460 \mathrm{~kW}$ ) used in this study.

Measurements of energy release rate during water spray suppression were correlated by assuming an exponential decay from the energy release rate at the time of initial water spray application. The time constant for this decay in energy release rate that provided the best fit of the data was $28 \%$ of the calculated time to extinction.

The significance of these results is that both time to fire extinction and energy release rate during suppression can be estimated for the purpose of hazard assessment fire modeling without detailed calculation. Of course there remains the assumption that all fires must be reduced to an equivalent wood crib fuel source in order to perform the analysis. 


\section{Thermal Model of Extinction}

Previous analysis and measurement of water droplet evaporation on non-porous high thermal conductivity surfaces were extended to both porous and ron-porous low thermal conductivity solids. The analysis of droplet evaporation on low thermal conductivity solids requires coupling of the liquid and solid phase conduction analysis. This was avoided in analysis of evaporation from metal surfaces by using a constant interface contact temperature. The prediction of droplet evaporation on non-porous surfaces uses a Green's function integration over transformed domains representing the droplet and the solid. Measurements using both porous and non-porous ceramic solids have shown that: (1) water droplet evaporation is quicker from porous solids than non-porous solids and, (2) thermal recovery time and volume of droplet influence are greater for porous solids compared to non-porous solid. An apparatus that irradiate solids from external heat sources above the surface, simulating heat flux from a flame, is being constructed. This apparatus will be used to extend the study of droplet evaporation into a geometry that closely simulates burning fuels.

\section{Reports and Publications}

Suppression of Wood Crib Fires With Sprinkler Sprays: Test Results, William D. Walton, National Bureau of Standards Interagency Report, NBSIR 88-3696, January 1988 .

Water Spray Suppression of Fully- Developed Wood Crib Fires in a Compartment, James A. Milke, David D. Evans, and Warren Hayes Jr., National Bureau of Standards Interagency Report, NBSIR 88-3745, June 1988.

"Progress in Modeling Sprinkler Performance," in U.S./Japan Government Cooperative Program on Natural Resources (UJNR). Fire Research and Safety. 9th Joint Panel Meeting of the UJNR Panel. May 4-8, 1987., Norwood, MA, David D. Evans, William D. Walton, and Bernard J. McCaffrey, National Bureau of Standards Interagency Report, NBSIR 88-3753, April, 1988.

Cooling Effect of a Single Evaporating Droplet on A Hot Semi-Infinite Metal Body, Marino di Marzo, Anil K. Trehan, and David D. Evans, National Bureau of Standards Interagency Report, NBSIR 87-3517, April, 1987.

Dropwise Evaporative Cooling of High Thermal Conductivity Materials, Marino di Marzo, and David D. Evans, Heat and Technology, Vol.5, No. 1-2, 1987.

"Evaporation of a Water Droplet Deposited on a Hot High Thermal Conductivity Solid Surface," Marino di Marzo and David D. Evans, Heat and Mass Transfer in Fire, HTD-Vol 73, A. K. Kulkarni and Y. Jaluria, editors, The American Society of Mechanical Engineers, New York, NY 10017, 1987

"Ceiling-Jet Flows," David D. Evans, SFPE Handbook of Fire Prevention, Phillip Di Nenno, editor, National Fire Protection Association, Quincy, MA, 1988

"Use of Computer Fire Models for Analyzing Thermal Detector Spacing," David W. Stroup and David D. Evans, Fire Safety Journal, Vol 14, 1988. 
Measurement of Droplet Size in Sprinkler Sprays, James R. Lawson, William D. Walton, and David D. Evans, National Bureau of Standards Interagency Report, NBSIR 88-3715, February 1988.

\section{Related Grants}

"Effects of Water on Ignition of Cellulosic Materials," Arvind Atreya and Indrek S. Wichman, Michigan State University.

"Transient Cooling of A Hot Surface"" Marino di Marzo, University of Maryland.

"A Study of the Suppression and Extinction of Fires by Water Sprays," Walter W. Yuen and Wilber Lick, University of California.

"Development of a Technique to Assess the Adequacy of the Municipal Water Supply for Residential Sprinkler Systems", John L. Bryan and James A. Milkie, University of Maryland. 
CENTER FOR FIRE RESEARCH

NATIONAL BUREAU OF STANDARDS

FY 88

Institution: Michigan State University

Grant No.: 60NANB5D0578

Grant Title: Effect of Water on Ignition of Cellulosic Materials

Principal Investigator(s): Drs. Arvind Atreya and Indrek S. Wichman

Department of Mechanical Engineering

Michigan State University

East Lansing, MI 48824-1226

Other Professional Personnel: L-S Tzeng, Graduate Research Assistant

M. Abu-Zaid, Graduate Research Assistant

NBS Scientific Officer: Dr. David D. Evans

Technical Abstract:

Introduction The study of ignition and extinguishment of combustible cellulosic materials is of great importance for fire safety because these materials constitute the bulk of the fuels in many building fires. Because of the porosity of cellulosics, water plays an important role from ignition to extinguishment. Absorbed water delays the onset of ignition and consequently retards the fire growth rate. Also, water is the most common agent used to extinguish such fires. Our results will be useful for determining onset of ignition, the fire growth rate and its subsequent containment by external application of water.

Piloted Ignition Experiments Measurements of the time required for ignition, surface temperature, mass evolution rate (fuel and water vapor) are made for different levels of external radiation and sample moisture content. The test facility is shown in Figure 1. The wood samples are instrumented with thermocouples, dried (at $105^{\circ}$ over phosphorous pentoxide), weighed, conditioned to a known relative humidity, weighed again (to determine moisture weight), then subjected to a known external radiant heat flux. The duct exhaust is continuously analyzed for water concentration and $\mathrm{CO}, \mathrm{CO}_{2}, \mathrm{O}_{2}$ and total hydrocarbons. Thus, for a given moisture content and external radiation the following measurements are made as a function of time (i) front and back surface temperatures, (ii) mass evolution rate of water vapor, (iii) production rate of combustible gases.

Theoretical Model of Piloted Ignition A simplified model of piloted ignition is analyzed numerically. The two-dimensional couples gas and solid phase problem is simplified by assuming that the mass evolution rate from the combustible is a known function of time and by employing a plane (not a point) ignition source; thus, the model problem is transient and one-dimensional. The effects of (i) ignition source location, (ii) fuel mass evolution rate from surface, and (iii) surface temperature of solid fuel were investigated. The experimentally-observed pre-ignition flashes are explained. A rational criterion for positioning of the pilot flame is proposed (at equilibrium diffusion flame location). Also, the minimum fuel flow rate, by itself, is found insufficient for predicting inset of piloted ignition - heat losses play an important role. Heat flux profiles, which are proportional to the local 
temperature gradient, are shown for the propagating premixed flame stage of piloted ignition in Figure 2. Note how quickly the premixed flame moves through the mixture.

Transient Cooling of Ceramic Solids by Droplet Evaporation The effect of a single droplet of pure water on transient cooling is studied experimentally. The solid surface temperature ranged from 75 to $200^{\circ} \mathrm{C}$; they were instrumented with surface and indepth thermocouples. For the non-porous solid these temperatures remain nearly constant, while for the porous solid a continuous decrease is observed during droplet evaporation. Thus, an energy sink exists in the porous matrix in the vicinity of the thermocouple. From the transient measurements of surface and indepth temperatures the following quantities were determined, (i) recovery time (larger for porous than non-porous), (ii) size of surface and indepth zones affected by droplet (larger for porous solid). Shown in Figures 3 and 4 is a comparison between indepth temperatures for porous and non-porous solids for an initial solid surface temperature of $100^{\circ} \mathrm{C}$ and a droplet volume of $30 \mu \mathrm{L}$.

\section{Reports and Papers:}

1. Tzeng, L.S., Wichman, I.S. and Baum, H.R., "A Combined Analytical-

Numerical Solution Procedure for Chemically Reacting Flows," Central State Meeting of the Combustion Institute, pp. 426-431 (1987).

2. Tzeng, L.S., Atreya, A. and Wichman, I.S., "A One-Dimensional Model of Piloted Ignition," submitted to Combustion and Flame (1988).

3. Abu-Zaid, M. and Atreya, A., "Transient Cooling of Hot Porous and NonPorous Ceramic Solids by Droplet Evaporation," submitted to Journal of Heat and Mass Transfer (1988).

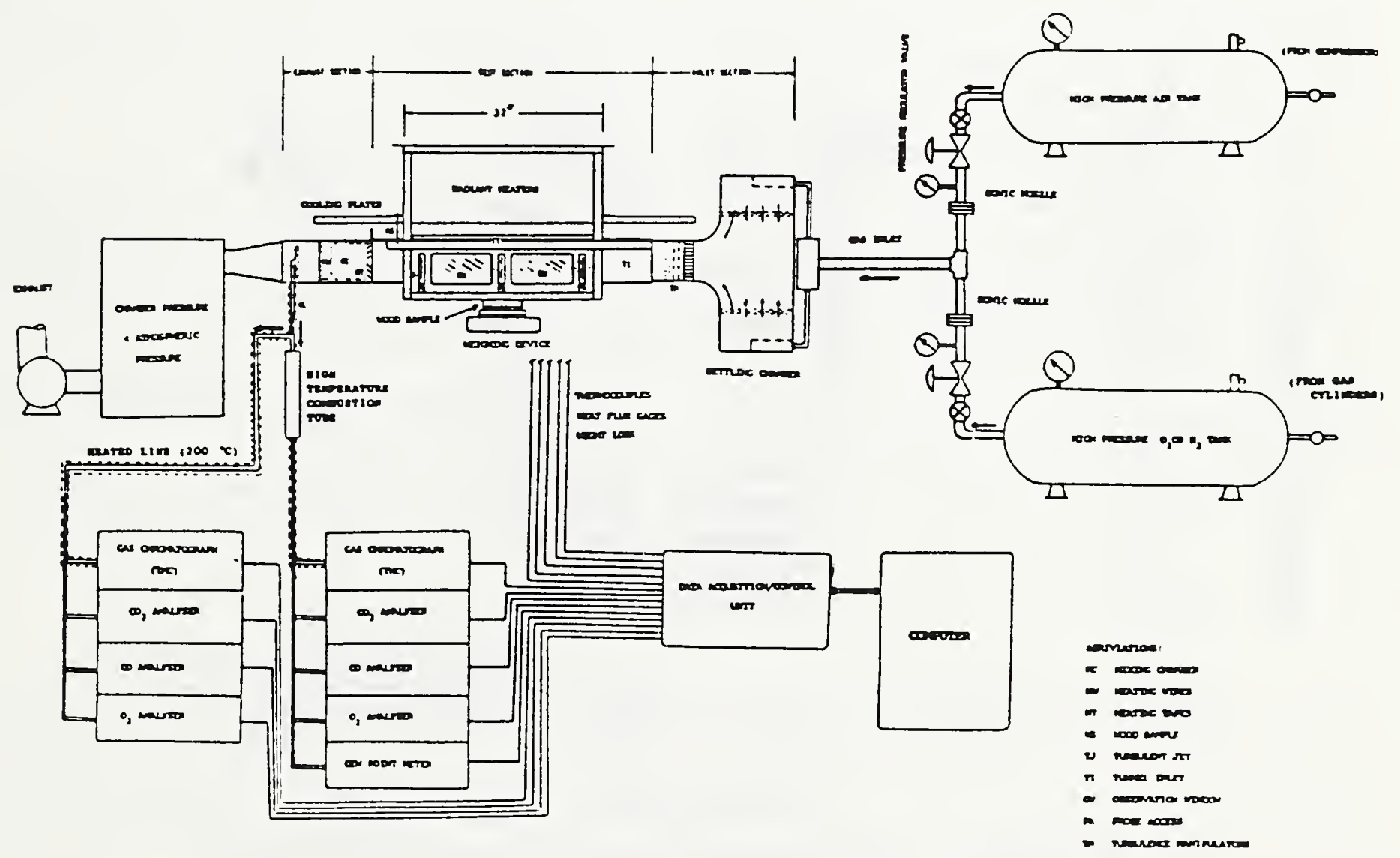

Figure 1. Schematic of the Experimental Apparatus 


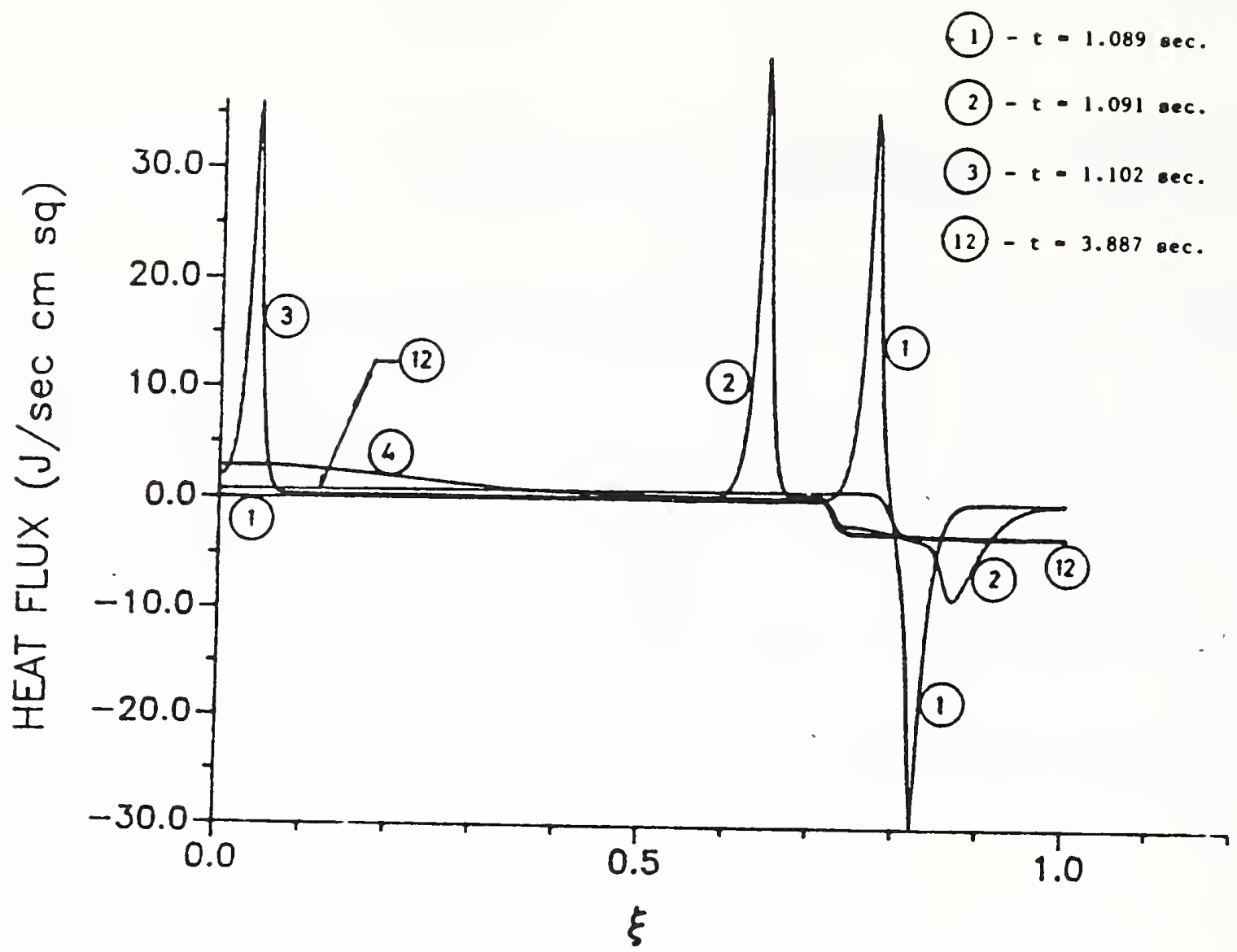

FIGURE 2: Heat flux profiles (proportional to temperature gradient) for the propagating pre-alxed flame during plloted ignition.

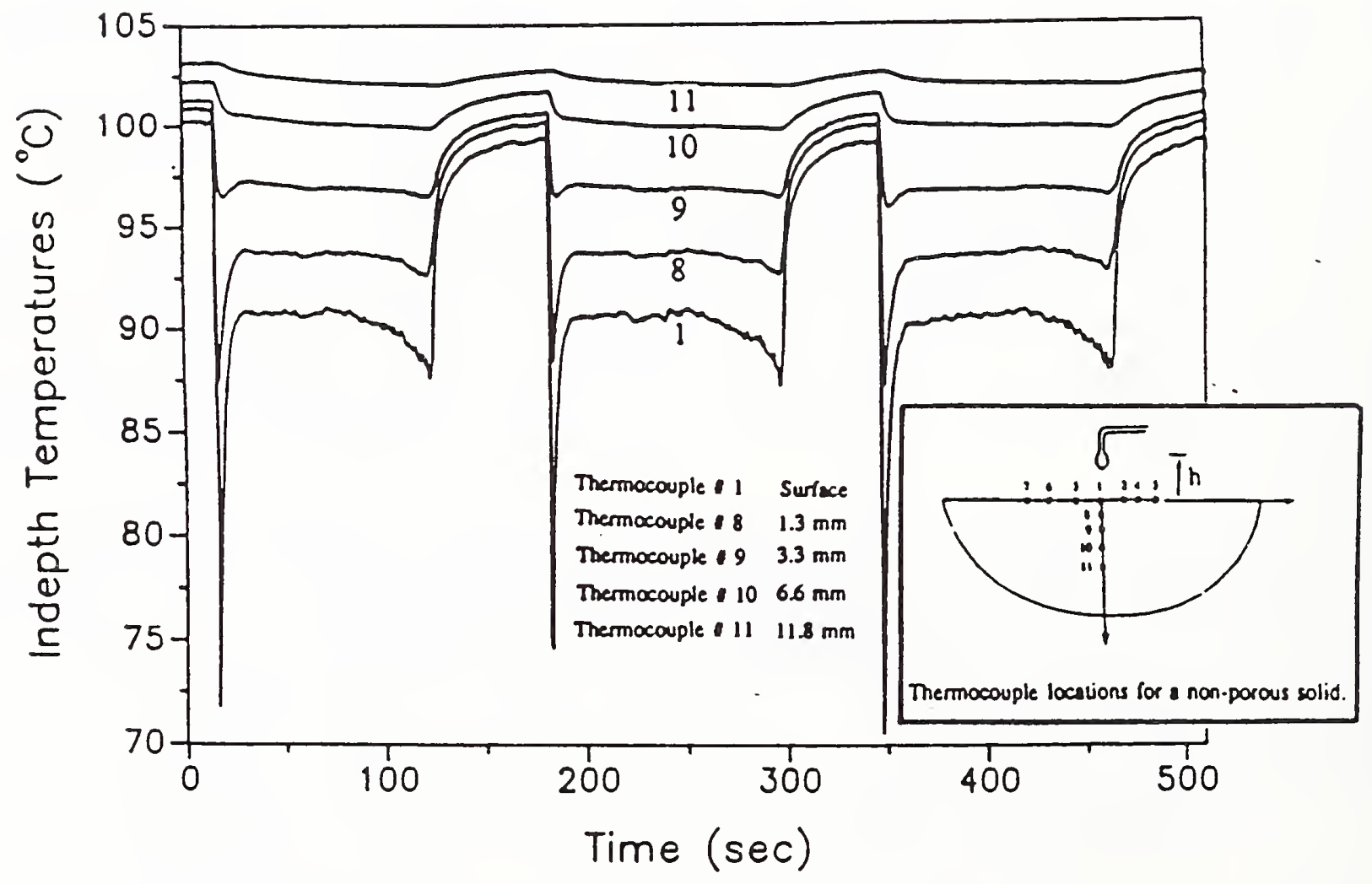

Fig. 3. Indepth temperatures versus time for o nonporous solid. Initiol solid surfoce temperoture $100^{\circ} \mathrm{C}$. Droplet volume $30 \mu \mathrm{L}$. 


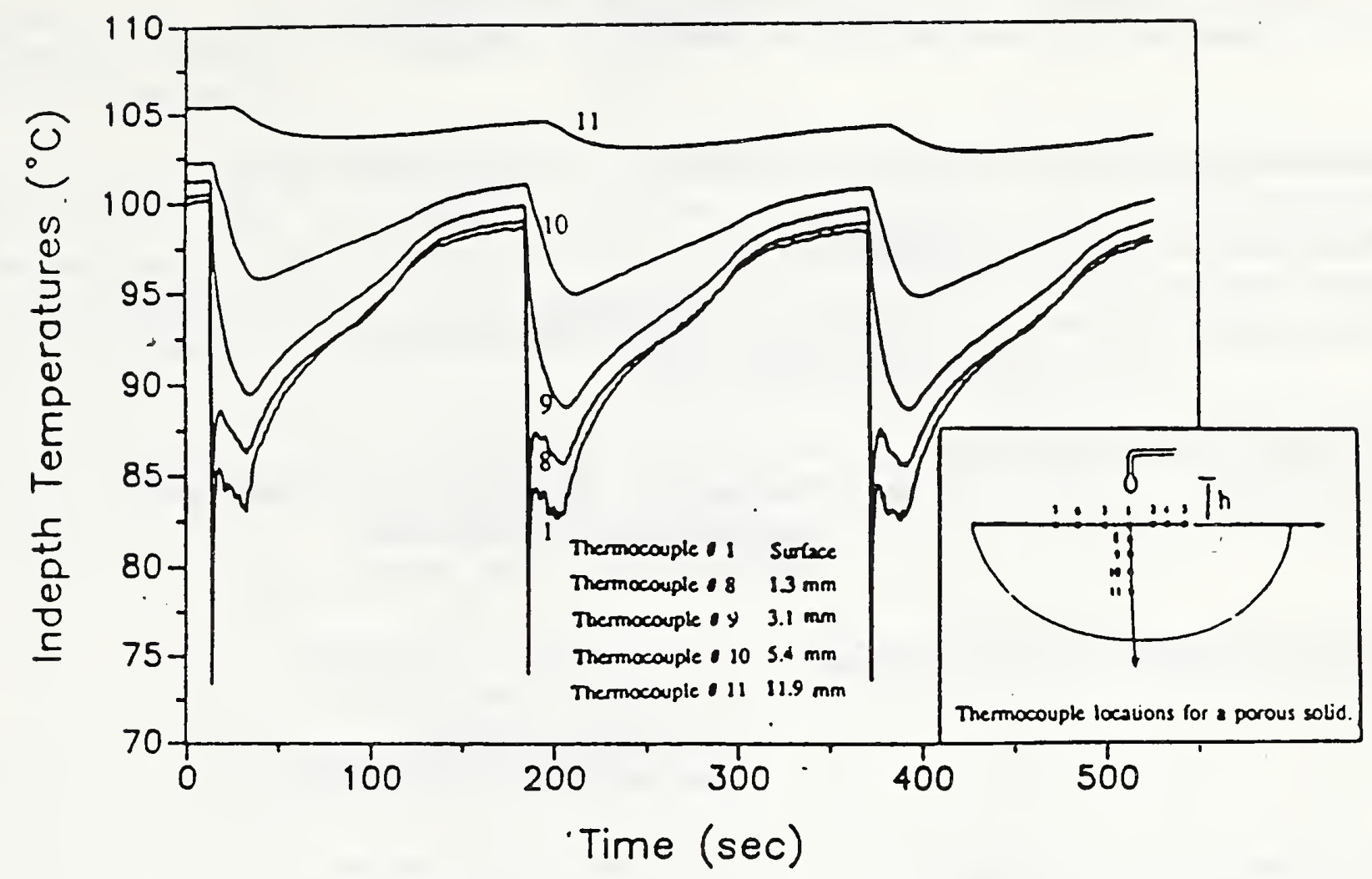

Fig. 4. Indepth temperatures versus time for a porous solid. Initial solid surface temperature $100^{\circ} \mathrm{C}$. Droplet volume $30 \mu \mathrm{L}$. 
Institution: The University of Maryland at College Park

Grant No.: COMM 70NANBSHOS25

Grant Title: Transient Cooling of a Hot Surface by Droplets Evaporation

Princ1pal Investiqator: Dr. Marino d1 Marzo

Mechanical Eng1neering Department

Untversity of Maryland

College Park, MD 20742

Other Professionel Personnel:

NBS Sc1ent1fic Officer:
F. Kavoos1, Graduate

Research Assistant

D. Madrzykowsk1,

Undergraduate Student

Dr. David D. Evans

\section{Technical Abstract:}

Introduction The phenomena associated with soldd fuel fire extinguishment processes are related to the evaporation of vater droplets deposited on hot solids. In the previous grant perlods ve successfully correlated the experimental results for droplets deposited on high thermal conductivity solids. Extensive experimental data for a lov conductivity material vere also collected. Further investigation of a different low conductivity solid vill be performed in the next grant period. The current effort 18 almed at developing a model to predict the complex thermal behavior of the liquid-solid interaction on these low conductivity materials.

Some detalled aspects of a small droplet generator vill be discussed in the following. The droplet size characteristic of sprinkler distributions is in the order of $1 \mu l$. These small droplet are strongly influenced by surface tension forces and special techniques must be developed to disengage them from the dispenser.

Evaporative Cooling Model The governing equation for the solld transient thermal behavior and 1 ts boundary conditions are:

$$
\frac{\delta T}{\delta t}=\alpha_{s} \nabla^{2} T
$$

The temperature gradient at the exposed solid surface $(\delta \mathrm{T} / \delta z)$ ls prescribed. At the solid-11quid boundary under the droplet, both the heat flux and the temperature are matched. For the liquid the governing equation is identical vith the exception of the different thermal 
diffusivity. The boundery condition at the exposed surface of the droplet is casted in terms of evaporative mass transfer as described in a previous paper [2].

The 1dea of using the Green's theorem to reduce the three dimensional nodalization to a simpler surface description of the phenomena 18 extremely appealing because ve are only interested on the area influenced by the droplet cooling at the surface of the solid. The general solution for the transient conduction equation 18:

$$
\begin{aligned}
& u(x, t)=\alpha \int_{0}^{t} \int_{S}[G(x, t, x \theta, t \theta)(\nabla u)-u(x \theta, t \theta)(\nabla G)] \times \hat{n} d \theta d t \theta \\
& +\int_{V} G(x, t, x \theta, \theta) u(x \theta, \theta) d v
\end{aligned}
$$

Note that $u$ is a simple transform of the temperature ' $T$ ' defined in such a vay that the volume integral is eliminated. The functions $G$ for the solid and the liquid are defined, in the general form, as:

$$
\begin{aligned}
G(x, t, x \theta, t \theta) & =(4 \pi \alpha t \theta)^{-1.5} e^{-\frac{(x-x \theta)^{2}+(y-y \theta)^{2}+(z-z \theta)^{2}}{4 \alpha t \theta}} \\
& +(4 \pi \alpha t \theta)^{-1.5} e^{-\frac{(x+x \theta)^{2}+(y+y \theta)^{2}+(z-z \theta)^{2}}{4 \alpha t \theta}}
\end{aligned}
$$

Further, this selections of $G$ are such that $(\nabla \mathrm{G})$. $\hat{n}$ at the solid surface 1s zero. This simplifies the surface integral at the solid surface and at the 11quid-solid interface. The integral on the exposed surface of the droplet vill contain both terms ( $G$ and $\nabla G$ ). At this time we have ldentifled the terms related to the solid surface only. In general ve can represent these terms as a surface integral of the product of a forcing function $F$, which is the temperature gradient normal to the solid surface, and of a velghing function $W$. In cylindrical coordinates this can be vritten as:

$$
u(r, t)=\int_{0}^{t} \int_{0}^{\infty} F(r \theta, t \theta) W(r, r \theta, t \theta) d r \theta d t \theta
$$

where, for the general case, W 1 s given by: 


$$
\begin{aligned}
& 2 \\
& W(r, r \theta, t \theta)=\frac{1}{.5} r_{0} \omega_{0}^{-1.5}\left(\frac{2 r r \theta}{4 \alpha t \theta}\right) e^{-\frac{(r-r \theta)}{4 \alpha t \theta}} \\
& (4 \pi \pi)
\end{aligned}
$$

For the particular cases $(r=0$ and/or $t=0)$, we obtain specific forms of the veighing function $W$ vhich are integrated analytically over the interval of concern. The interval of concern 18 determined by the grid size ' $\epsilon$ ' and by the time step ' $T$ ' accordingly. These speciflc expressions for $W$ are:

$$
\begin{aligned}
& W(r \theta=r, t \theta)=r \text { to }{ }_{0}^{-1}\left(\frac{2 r^{2}}{4 \propto t \theta}\right) \operatorname{erf}\left[\frac{\epsilon}{.5}\right] \\
& \text { (16 \& to ) } \\
& W(r, r \theta, \theta)=\frac{1}{2 \pi}(r \theta / r)^{.5} E\left[\frac{(r-r \theta)}{4 \alpha T}\right] \\
& W(r \theta=r, \theta)=2\left(\frac{\alpha T \cdot .5}{\pi}\right) \operatorname{erf}\left[\frac{\epsilon}{.5}\right]+\frac{\epsilon}{2 \pi} E\left[\frac{\epsilon^{2}}{16 \alpha \tau}\right] \\
& \left(\begin{array}{lll}
16 & \propto & 7
\end{array}\right)
\end{aligned}
$$

Preliminary results for the solid vere obtained and compared favorably vith the previously published data for aluminum [ri].

Droplet dispenser The average size of the droplet generated by a sprinkler system is in the order of one $\mu l$. The droplet size used to investigate the cooling effect due to the evaporative cooling ranges from 10 to $50 \mu 1$. This size range 18 most convenient for accurate experimental measurements because such big droplets are easy to generate and their evaporation time is long enough to carefully monitor the transient heat and mass transfer processes. The first difficulty that arises in the generation of one $\mu 1$ droplets is due to the strong surface tension forces. The droplet does not leave the dispenser and clings to 1t. We have abserved that the minimum size that leaves a thin needle or fiber is of the order of $10 \mu \mathrm{l}$. The addition of a surfactant brings this minimum size down to $3-5 \mu l$. Hovever, the purity of the vater is compromised and the experimental data loose their significance.

The droplet must have some momentum in order to disengage from the dispenser tip. He have designed and bulld a dispenser in which a thin disk is imparting momentum to the liquid and it is pushing it through a nozzle. A schematic of the conceptual design is given in Fig. 1. Initially ve choose a piezoelectric activated disk, but the incompatibility of vater vith the plezoelectric material required the 
system to be sealed. The constraints due to the leak proof system vere such to almost completely restrain the piezoelectric motion.

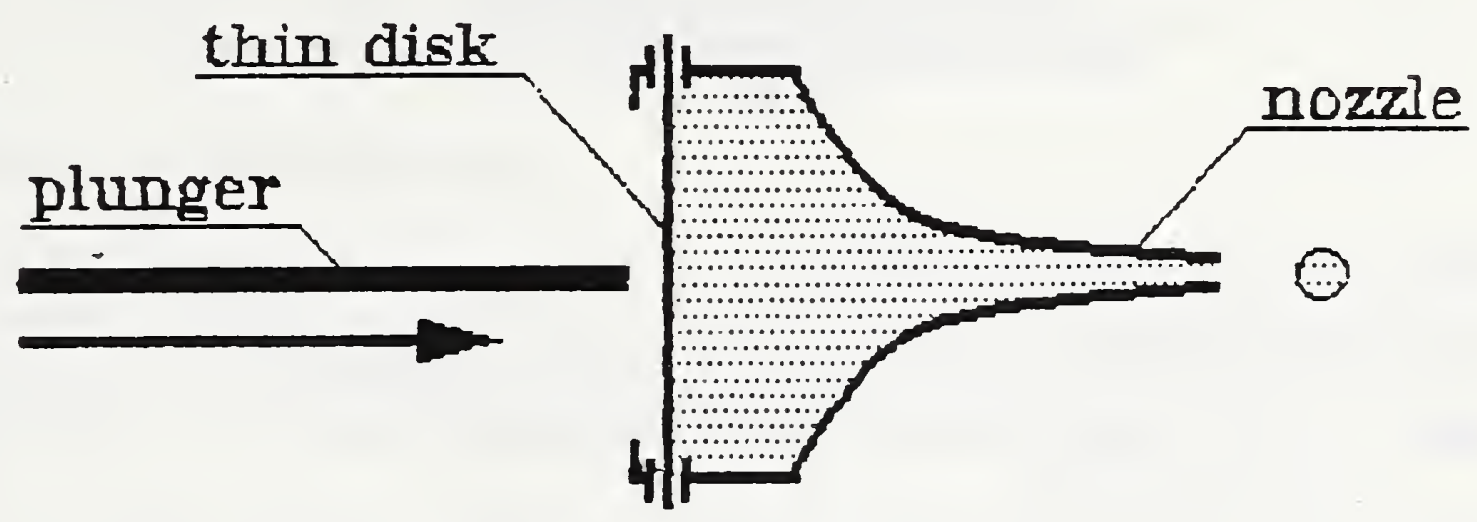

Figure One - Droplet Generator Design Conceptual

In order to pulse the thin disk ve used a mechanical spring-loaded plunger. This device ras difficult to control and ras used to explore the feasibility of a mechanical pulse generator for the thin disk. Preliminary results indicated that very small droplets $(0.01 \mu 1)$ vere generated and they vere scattered over a 12 millimeter radius. The exact size and position of the droplet must be controlled in order to perform the necessary measurements during the transient. Therefore, ve vill design a different device to impart a controlled pulse to the thin disk. This new pulse generator vill be activated by a solenold driven rod attached to the thin disk. The size of the dispenser nozzle ras 150 microns in diameter. We are bullding various nozzles of larger size to study the effect of the nozzle diameter on the droplet size.

Acknorledgements Dr. H. Baum suggested the approach to the numerical computation of the coupled liquid-solid evaporative cooling problem. His guidance has been invaluable for the progress of this research. The Computer Science Center of the University of Maryland at College Park provided support for the computational expenditures.

\section{References}

r1. M. di Marzo, D. D. Evans, "Dropvise Evaporative Cooling of High Thermal Conductivity Materials", Heat and Technology, Vol. 5, No. $1-2$, pp. 126-136, 1987

Reports and Papers:

1. M. di Marzo, Z. Y. Wang, W. H. Meng, "Transient Cooling of a Hot Surface by Droplet Evaporation", National Bureau of Standarda Report, NBS-GCR-87-534, 1987

2. M. di Marzo, D. D. Evans, "Evaporation of a Water Droplet Deposited on a Hot High Conductivity Solid Surface", 24th ASME/AIChE National Heat Transfer Conference, Pittsburgh, Pennsylvania, HTD Vol. 73, pp. 11-18; Also to appear in Journal of Heat Transfer. Transaction ASME

3. W. H. Meng, Z. Y. Wang, M. di Marzo, D. D. Evans, "Single and Multi Droplet Evaporative Cooling", Combined Meetings: Esstern Session of the Combustion Institute and CFR Annual Conference on Fire Research, NBS - Gaithersburg, Maryland, pp. 42.1-42.4, 1987 


\section{CENTTER FOR FIRE RESEARCH \\ NATIONAL BUREAU OF STANDARDS \\ FY 87}

Institution: The University of California

Grant No: 60NANB6D0651

Grant Title: A Study of the Supression and Extinction of Fires by Water Sprays

Principal Investigators: Professors W. W. Yuen and W. Lick Department of Mechanical Engineering University of California at Santa Barbara Santa Barbara, California, 93106

NBS Scientific Oficer: Dr. Bernard J. McCaffrey

\section{Technical Abstract}

\section{INTRODUCTION}

The objective of this study is to determine the fundamental mechanisms by which the spraying of water leads to fire supression and extinction. During this reporting period, the research concentrated on three specific tasks: (1) the numerical modelling of the general and localized circulation in a room as dependent upon door and window openings; (2) a detailed analysis and description of the dynamics of water spray as influenced by the fluid dynamics; and (3) the thermal quenching of a flame due to the presence of chemically-inert species. The first two tasks contribute to the understanding of the "survivability" of the water sprays in a fire environment after injection. The third task contributes to the understanding of the interaction between the water droplets/vapor and the reacting species in a combustion/extinction process.

\section{MODEIJING OF CIRCUIATION IN A ROOY WTTH DOOR AND WIDOW OPENINGS}

A quite general numerical model of the time-dependent dynamics of a compressible, viscous, heat-conducting, chemically-reacting mixture of gases has been developed and applied to the problem of natural heat convection in a room with door and window openings. A unique feature of the model is its treatment of the conditions at the doors and windows, where open boundary conditions (Licks, et. al. 1986, 1987). must be specified. Numerous calculations have been made and are being made to investigate the general dynamics of natural convection in a room with specific emphasis on the effects of door and window opening.

A preliminary calculation of this type, primarily to test the open boundary condition, is shown in Figures 1 and 2. A heat source at the middle of the left wall causes the air to rise and a ceiling jet to develop. On the right, the hot air escapes from the top and cool air is drawn in at the bottom. A steady-state eventually develops and it can be shown that the open boundary condition (in its 
nonlinear formulation) works extremely well. This type of calculation is continuing so as to investigate the effects of various flow parameters.

\section{DYNAMICS OF TATER DROPLETS}

The model described in section 2 is used simply to describe the general fluid dynamics. Of primary interest is the dynamics of the water droplets as influenced by the fluid dynamics and the influence of the water droplets on the fluid dynamics. A general numerical model of this interaction is being developed.

Both the fluid dynamics and the droplet dynamics are developed ty an Eulerian procedure. For complex problems of droplet-fluid dynamics interaction, this seems most appropriate and numerically efficient. The basic conservation equations for the water droplets have been written in a form identical to the fluid dynamics equations used in the model described in section 2 and are presently being included in that model. In its most general form, this model can then be used to describe the dynamics of water droplets of varying sizes and the droplet's size changes as a function of evaporation and heat transfer.

\section{THERYAL QUENCHING BY CHEMCALLY-INERT SPECIES}

Since water is not expected to be chemically reactive with most common fuels, the supression and extinction of fire by water is primarily a thermal quenching phenomenon as noted by previous investigators (Rasbash 1962, Grove et.al. 1981, Williams 1981). A survey of the literature, however, shows that while extensive amount of data have been collected on the relative effectiveness of various gaseous/liquid agents on thermal quenching (Friedman and Levy 1963, Seshadri and Williams 1975, Kent and Williams 1975, Tucker et.al. 1981), a fundamental non-empirical theoretical study of this important phenomenon has not been reported. The development of such a theoretical model is a major objective of this research program.

A laminar diffusion flames generated by two opposing flows of fuel and oxidizer is selected as the basis of the present extinction study. The geometry is shown in Figure 3. This geometry is selected because it is one of the few cases for which conservation equations for mass, momentum and energy can be readily written from first principle with no empirical constants. Assuming constant thermophysical properties, density and a unit Lewis number, numerical and approximate solutions to this problem have been reported by many investigators (Fendell 1965, Linan 1974, Krishnamurthy et.al. 1976). Many of the reported experimental studies on extinction (Friedman and Levy 1963, Seshadri and Williams 1975, Kent and Williams 1975, Tucker et.al. 1981) were also performed on this opposing flow configuration.

The mathematical development in the previous works is generalized in the present work to allow a variation of the specific heat of the reactants and products as a function of composition and temperature. Numerical results are generated for a pure fuel stream (methane) and oxidizer streams consisting of oxygen and different inert gases of various proportions.

Typical ignition characteristics generated for three inert gaseous species $\left(\mathrm{N}_{2}, \mathrm{H}_{2} \mathrm{O}\right.$, and $\mathrm{He}$ ) with different specific heats are presented in Figure 4a. At $600 \mathrm{~K}$, the specific heat for $\mathrm{N}_{2}, \mathrm{H}_{2} \mathrm{O}$ and $\mathrm{He}$ are $1.0 \mathrm{~J} / \mathrm{gm}-\mathrm{K}, 2.0 \mathrm{~J} / \mathrm{gm}-\mathrm{K}$ and 5.2 $\mathrm{J} / \mathrm{gm}-\mathrm{K}$ respectively. As expected, for a given oxygen mass fraction in the oxidizer stream, the mixture ignites if the dimensionless Damkohler number (which 
is a ratio of the characteristic time of the flow to the characteristic time of the reaction) exceed a critical value. For a given inert gas, a decrease in the oxygen mass fraction in the oxidizer stream reduces the reaction rate, the critical Damkohler number required for ignition increases. For the same oxygen mass fraction, a mixture with an inert gas of higher specific heat has a lower mixture temperature and therefore lower reaction rate. The critical Damkohler number required for ignition also increases.

Since the Damkohler number is inversely proportional to the characteristic flow velocity, numerical data presented in Figure 4 a can be presented in terms of the characteristic flow velocily, $u$, as shown in Figure $4 b$. $u_{0}$ is a characieristic velocity corresponding to the ignition of an oxygen/nitrogen mixture with $50 \%$ oxygen mass fraction. It is interesting to note that Figure $4 \mathrm{~b}$ is qualitatively similar to some experimental extinction curves. Since $u_{0}$ is the only adjustable parameter in Figure $4 \mathrm{~b}$, the present results suggest that measurement of the extinction curves for different inert species and oxygen mass fraction can be an effective mean in determining the various reaction constants for the reaction.

In the next reporting period, additional numerical results will be generated for other fuels and inert gases. The model will also be expanded to allow for variable density and Lewis number different from unity. Based on the model, the effect of liquid inert species on the reacting mixture will be addressed. Specifically, the fire supression/extinction characteristics of water spray will be illustrated. 


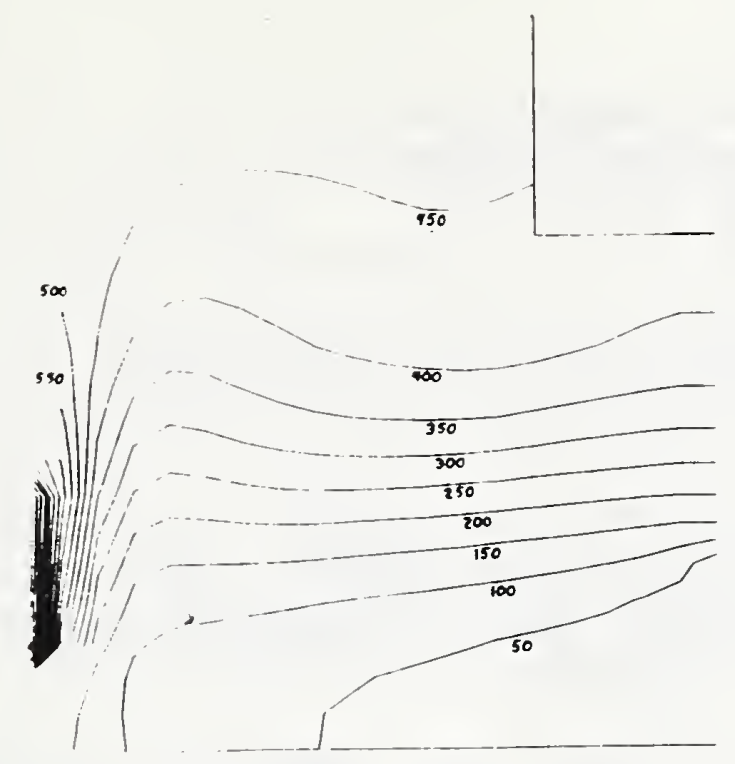

Fig. 1. Computed Isotherms in an Enclosure with a Hot Spot $(1000 \mathrm{C})$ at the Wall and an Opening.

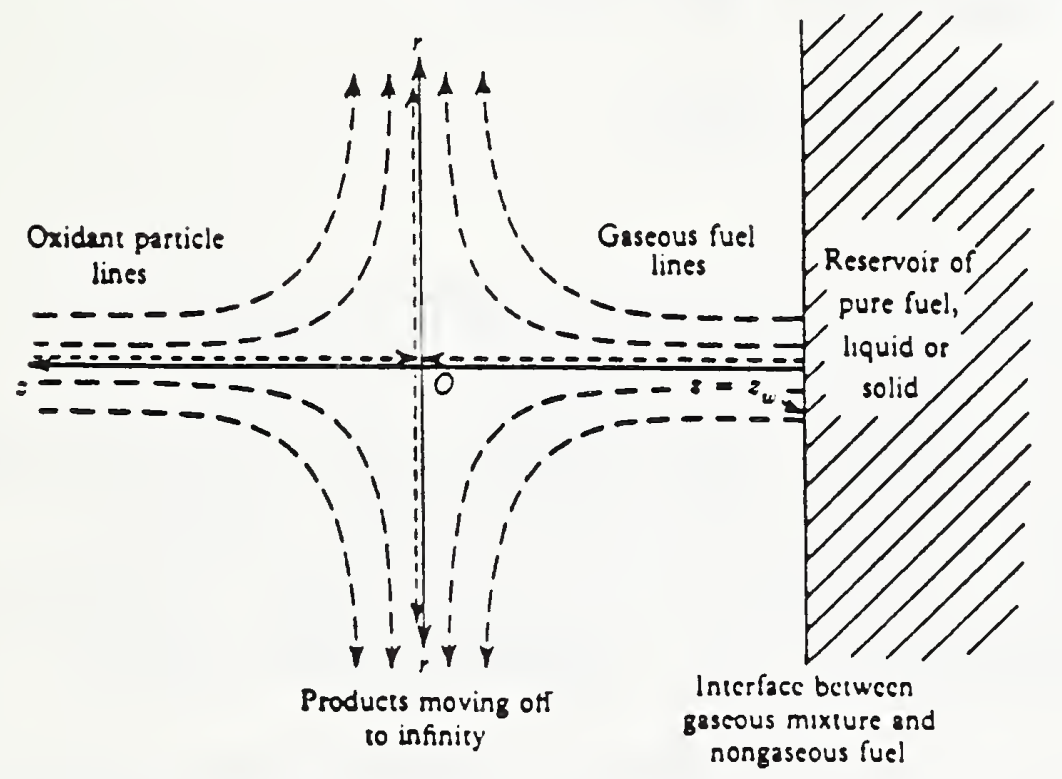

Fig. 3. Oxidant, Fuel, and Product Particle Lines in an Axially Symmetric Stagnation-Region Flow.

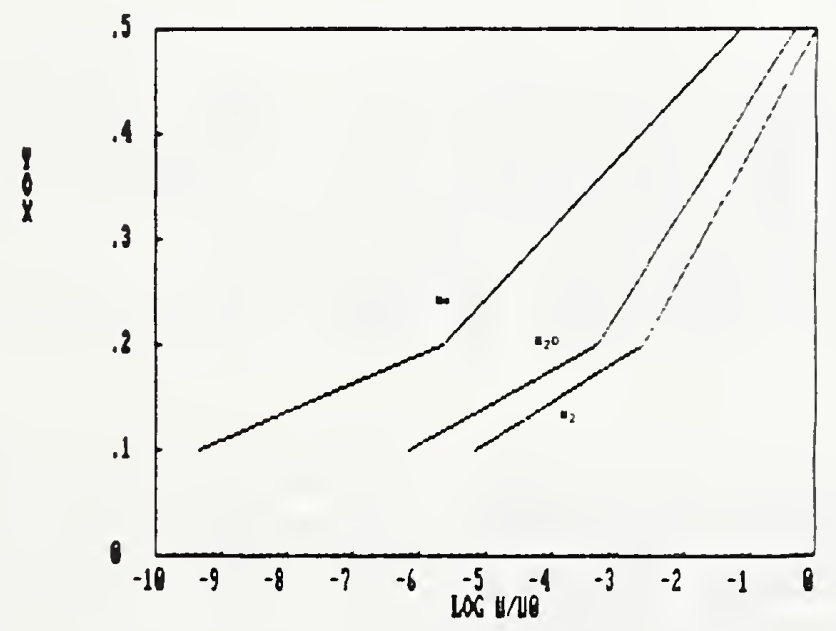

Fig. 2. Computed Flow Field in an Enclosure with a Hot Spot $(1000 \mathrm{C})$ at the Wall and and Opening.

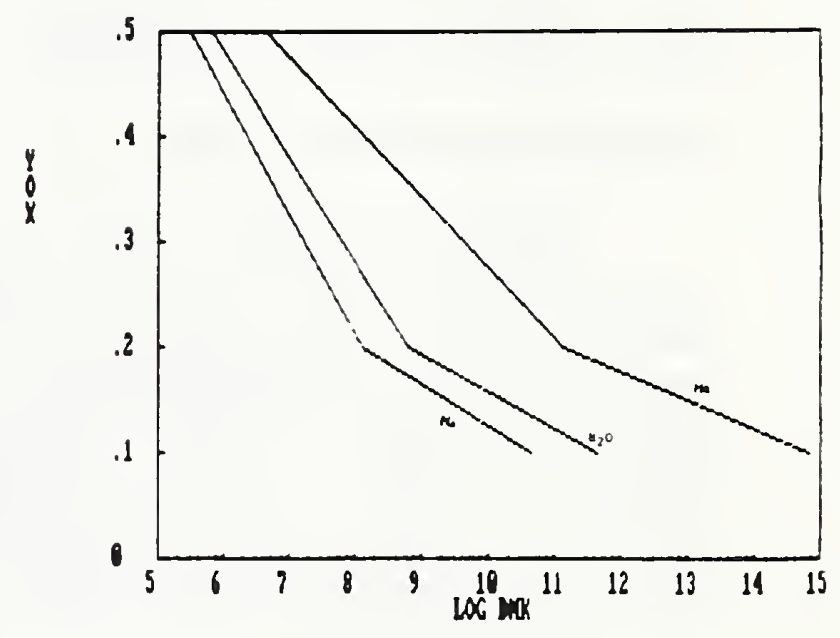

Fig. 4a. Critical Damkohler Number Required fo: Extinction for Oxidizer Streams with Different Oxygen Mass Fraction and Inert Gas.

Fig. 4b. Critical Characteristic Flow Velocity Required for Extinction for Oxidizer Streams with Different Oxygen Mass Fraction and Inert Gases. 


\section{CENTER FOR FIRE RESEARCH \\ NATIONAL BUREAU OF STANDARDS \\ FY 88}

Institution: The University of Maryland at College Park

Grant No.: $\quad$ 60NANB8D0814

Grant Title: Development of a Technique to Assess the Adequacy of the Municipal Water Supply for Residential Sprinkler Systems.

Principal Investigator: Dr. John L. Bryan and James A. Milke Fire Protection Engineering Department University of Maryland College Park, MD 20742

NBS Scientific Officer: Dr. David D. Evans

Technical Abstract:

This project is a continuation of work examining the capability of a municipal water supply system to supply a residential sprinkler system. The initial phase of work emphasized an assessment of the costs associated with alleviating deficiencies in the capability of the municipal water supply system. The current phase has focused on an evaluation of the water supply in residences as provided by the municipal system in the Baltimore, Maryland - Washington, D.C. metropolitan area.

In the first phase, simple cost effective techniques for alleviating water supply deficiencies in a residential sprinkler system installed in a one or two-family dwelling have been examined. The equipment associated with compensating for a inadequate municipal water supply using water storage tanks, booster pumps, and large diameter pipe have been investigated for efficiency and cost effectiveness.

Hydraulically-designed, NFPA 13D, residential sprinkler systems in seven residences were used to carry out this research. The seven selected floor plans of one and two-family dwellings included in this project were representative of a wide range of designs currently being utilized in actual residential

subdivisions under construction at the time of the project in the Baltimore-Washington area.

The simple technique for determining the installation cost of a sprinkler system was formulated following an analysis of the sprinkler system versus the key design features of the dwelling. Emphasis was placed on developing a technique which was accurate and easy to apply with minimal time and effort. 
In the second phase the evaluation consisted of determining the water supply characteristics at selected residences in the metropolitan area. Water flow measurements were conducted using characterized residential flow devices, e.g. faucets and hose bibbs, which were in-place in the selected residences. An elementary hydraulic analysis was used to determine the characteristics of the domestic water supply at the water meter, based on the results of the flow test. The adequacy of the municipal water supply system can be assessed with a knowledge of the water supply characteristics at the water meter and the demand requirements of the residential sprinkler system also determined at, the water meter. 

PART I. Basic Research and Tools for "Engineered" Fire Safety (In-House Projects and Associated Grants Funded by (NIST)

F. TECHNOLOGY TRANSFER 


\section{CENTER FOR FIRE RESEARCH \\ PRIORITY PROJECT}

\section{TECHNOLOGY TRANSFER}

\section{Professional Personnel}

James H. Winger, Project Leader

Nora H. Jason, Technical Information Specialist

W. Doug Walton, Fire Protection Engineer

\section{Project Objective}

Facilitate the transfer of the scientific and technical output of the Center to the user community.

\section{$\underline{\text { Scope }}$}

The transfer of the results of fire research to the fire safety community has been identified in several forums as one of the most important and most neglected responsibilities of fire research organizations. Included in this responsibility is the obligation to effectively communicate with various segments of the fire safety community to identify their needs, provide information on the progress and capabilities of fire research, and to provide them with the outputs of research. The products of fire research need to be tailored to address identified community needs. Training in the use and usefuliness of the outputs of fire research is an essential part of this effort. Easily available information such as provided by the fire research library and the computer bulletin board is important to the fire safety community.

\section{Technical Accomplishments}

1. Conducted a very successful symposium on fire protection engineering methods with the Society of Fire Protection Engineers to recognize the publication by them of the first handbook on fire protection engineering. conference was attended by over 200 engineers, scientists, and other fire safety practitioners from around the world.

2. The Fire Research Information Service (FRIS) has over 30,000 fire research reports, books, video tapes, etc. in its collection and is growing at the rate of almost 2000 items per year. This is the most complete fire research library in the world. FIREDOC, the computerized bibliographic system in FRIS, has over 14,000 entries. FRIS is open to the public and FIREDOC is available for searching both on site and by telephone. This service is used by many individuals and organizations.

3. A first version prototype fire safety education computer game was developed based on the output of CFR developed fire models to demonstrate the potential for educational materials based on the results of fire research. Further improvements are needed prior to dissemination to the education community. 
4. The CFR computer bulletin board was established to provide access to all members of the fire research community, including those in remote sites.

Since its inception last year, it already has over 800 users from around the world including engineers, fire services, consultants, materials and products manufacturers, and others. These users are requesting and receiving information on the Center's activities such as meetings and workshops, recent publications, downloading of CFR computer software, and more.

5. A first meeting of leaders of international fire research organizations was held that has led to more effective communications, cooperation, and collaboration. Further meetings are now planned.

6. CFR developed technologies were presented at the annual meetings of the NFPA and the International Association of Fire Chiefs, and in many other forums. 
PART II. Timely Response to Current Fire Problems

(Projects Funded by Other Agencies and Private Sector Organizations)

A. FIRE/MATERIALS INTERACTION 
CENTER FOR FIRE RESEARCH

OTHER AGENCY PROJECT - 1988

\section{RADIATIVE IGNITION IN MICROGRAVITY ENVIRONMENT}

Funding Agency: NASA

Project Leader: Takashi Kashiwagi

Project Objective:

The objective of this study is to develop a theoretical model capable of predicting the radiative ignition of a cellulosic material ( for example, filter-paper) and subsequent flame spread over the sample in microgravity environment. This study is to lead to applications such as fire safety for spacecraft by understanding and predicting ignition and subsequent flame spreading behavior.

Technical Accomplishments:

The flow pattern and the temperature distribution in the gas phase during the preheating period, which is controlled by a balance between the thermal expansion of the gas due to the heat addition from the heated surface to the gas phase and conduction away from the heated region, were calculated first. The results were obtained analytically for early time and numerically for most times of interest in the experiments envisaged for this study. The additional complexity of convective mass transfer of the evolved degradation products from the sample surface to the gas phase is currently being incorporated into the preheating model. The characterization of thermal and thermal oxidative degradation of the cellulosic material is on progress including the measurements of $\mathrm{CO}, \mathrm{CO}_{2}$, and $\mathrm{H}_{2} \mathrm{O}$ in the evolved gases. The construction of an ignition chamber for radiative ignition experiments in a normal gravity has been completed. The calibration of the radiant flux distribution and preliminary ignition experiments are being conducted. Shadowgraph photography setup to observe ignition events is being assembled. The overall design of the flow reactor is completed and some of its components, for example high temperature flow settling chamber, are being constructed.

Publications:

H. Baum, T. Kashiwagi, and C. di Blasi, "Radiative Ignition of Solid Fuels in a Microgravity Environment - The preheating Problem" to be presented at 1988 Eastern States Combustion Meeting, December 1988. 
Funding Agency: U.S. Dept. of the Navy

Project Leader: James E. Brown

Project Objective:

To develop a bench-scale methodology for evaluating the fire performance properties of fiber-reinforced composites in order to assist the Navy in selecting composite materials for use on ships.

Technical Accomplishments:

1. Combustion Sensitivity Index Methodology

The dependence of rate of heat release (RHR) on external flux was determined for a broad range of materials under constant fluxes indicative of small and large (flash over) fires. The dependence is expressed as a thermal sensitivity index for RHR averaged over various periods of the flaming combustion. Additionally, the external flux requirement for sustained flaming combustion is ascertained.

2. Ignitability

Ignition delay time as a function of external radiant heating was determined over a broad range of fluxes. Minimum external fluxes required for ignition in reasonably finite periods can be predicted.

3. Testing of Materials

Fire testing of Navy candidate composite materials for surface ships was initiated. The results from these tests are being used to propose requirements for submarine applications.

Reports and Publications:

Report of Test "Combustion Characteristics of Fiber Reinforced Panels" J.E. Brown, Report No. FR3970, National Bureau of Standards, April 1987.

Report of Test "Cone Calorimeter Tests of Two Composite Materials" E. Braun and J. Didion, Report No. FR3973, National Bureau of Standards, November 1987.

"Cone Calorimeter Evaluation of the Flammability of Composite Materials", J.E. Brown, E. Braun, and W.E. Twilley, NBSIR 88-3733, National Bureau of Standards, March 1988.

"Cone Calorimeter Method for Determining the Flammability of Composite Materials" J.E. Brown, Proceedings of the fourth Annual Conference on Advanced Composites: "How to Apply Advanced Composites Technology" Dearborn, MI 13-15 Sept. 1988, pp. $141-150$. 


\section{CENTER FOR FIRE RESEARCH \\ OTHER AGENCY PROJECT - 1988}

FIRE PROPERTIES OF CANDIDATE MATERIALS FOR COMPOSITE DECKHOUSE

Funding Agency: Navy

Project Leader: T. Ohlemiller

Project Objective:

To develop a complete characterization of the flammability behavior (ignitability, lateral flame spread, upward flame spread) of composite materials which are candidates to be used in deckhouse structures aboard Navy ships. This characterization is to employ a combination of bench scale, intermediate scale and full scale tests.

Technical Accomplishments:

The Lateral Ignition and Flame Spread Apparatus (LIFT) was used to assess the heat-flux dependent ignition delay time, the minimum heat flux for ignition, flux-dependent lateral flame spread rate and minimum flux for lateral spread across four composite materials. In addition, these test results were used to infer values for certain empirical parameters which appear in simplified models for ignition and lateral flame spread. It was determined that these types of materials can exhibit differing flammability behavior at sample edges and at areas away from edges. This is apparently due to a tendency for the gases generated within the sample interior during heating to seek the path of least resistance in exiting from the sample; that path is frequently out through the sample edge rather than out through the sample face. Quantification of these effects will be pursued in the future together with characterization of other flammability measures on these materials. 


\title{
CENTER FOR FIRE RESEARCH \\ OTHER AGENCY PROJECT - 1988 \\ BURNING CHARACTERISTICS OF COMBAT SHIP COMPARTMENTS \\ AND VERTICAL FIRE SPREAD
}

\author{
Funding Agency: Navy (Naval Reserch Laboratory) \\ Project Leader: Daniel Gross
}

\section{Project Objective}

To perform a preliminary parametric computer modeling study of fire growth in combat ship compartments with emphasis on vertical fire spread as a result of heat transfer through metal decks.

\section{Technical Accomplishments}

Using the computer model FIRST (with some enhancements), estimates of fire development were provided over a wide range of variables, including compartment size, vent size, combustible load and compartment enclosure materials. Estimates were made of the heat transmitted through steel and aluminum decks and of potential ignition of combustible contents in compartments above the fire-involved compartment, as well as the effect of added thermal insulation. 


\section{CENTER FOR FIRE RESEARCH \\ OTHER AGENCY PROJECT - 1988 \\ PREDICTION OF THERMAL DEGRADATION AND HEAT RELEASE FROM AIRCRAFT COMPOSITES}

Funding Agency: Federal Aviation Administration

Project Leader: William J. Parker

Project objective:

Develop a theoretical and/or empirical or estimating technology for predicting an aircraft cabin panel's heat release rate in the OSU calorimeter.

\section{Technical Accomplishments:}

A preliminary model has been constructed to calculate the temperature distribution throughout an aircraft cabin panel. The panel consists of a Nomex honeycomb with facing sheets on each side. These sheets are of either fiberglass or carbon fibers and are impregnated with either epoxy or phenolic. It is assumed that the temperature is uniform in each facing sheet and that the heat transfer through the interior is by solid phase heat conduction through the walls of the cells and by gas phase heat conduction and thermal radiation through the cavities. The mass loss rate at each depth is calculated from an Arrhenius equation using measured kinetic constants and the local temperature and density. The heat release rate is determined by multiplying the local mass loss rate by the local heat of combustion of the volatiles and integrating over the thickness of the panel. Most of the thermophysical and thermochemical properties of the individual components were determined using the TGA, the DSC, the PYROCAT, and the oxygen bomb calorimeter. Some of the burning rate measurements have been completed. The heat release rates at $35 \mathrm{~kW} / \mathrm{m} 2$ have been measured in both the Cone Calorimeter in CFR and in the OSU Calorimeter at the FAA Technical Center in Atlantic City.

Publications: None 
Funding Agency: Carpet and Rug Institute, American Textile Manufacturers Institute, and the Man Made Fiber Producers Association

Project Leader: William J. Parker

Project Objective:

To provide a technical basis for recommending changes in the ASTM E-648 Flooring Radiant Test which would lead to a substantial reduction in the coefficient of variation for carpets.

\section{Technical Accomplishments:}

This project started with a review of an industry sponsored round robin involving five laboratories. The flame did not spread away from the ignition area for about half of those specimens, thus yielding a high critical radiant flux (CRF). However, other specimens of the same carpet spread flame over significant distances thus yielding considerably lower values of the CRF. Thus, uncertain propagation was identified as one of the problems, though not the only one, leading to high coefficients of variation. In the tests run on the same carpet at NBS the pilot was always sufficient to ignite the carpet and sustain a flame. However, the local flame that was produced experienced some difficulty moving down the specimen against the convectively driven air flow. A line burner which ignites the specimen over its entire width was constructed to replace the single flame jet used as the original pilot. This provides a longer, more stable flame front with one dimensional spread starting at the time of ignition. A coefficient of variation study was then made on eight carpets in order to pick one with the lowest coefficient of variation for use in a parametric study. This study which is just starting is systematically examining the effects of radiant flux, preheat time, air flow, adhesive mounting board, backing board, specimen orientation and the purity of the gas used by the radiant panel. The selected carpet is a $50 \mathrm{oz} / \mathrm{yd}^{2}$ cut pile Nylon 6,6 staple.

Publications: None 
CENTER FOR FIRE RESEARCH

OTHER AGENCY PROJECT - 1988

\section{NFPA 701 MULTIPLE LAYER DRAPERY FLAMMABILITY}

Funding Agency: ATMI, MMFPA, Wool Bureau

Project Leader: Sanford Davis

\section{Project Objective:}

To develop a flammability test method for multiple-layer fabrics, particularly curtains and drapes for inclusion in NFPA 701. The test method will correlate to full-scale multiple-layer fabric fire behavior.

\section{Technical Accomplishments:}

Working with the textile industry, four fire-retardant drapery fabrics (cotton, polyester, wool, and modacrylic) and two fire-retardant linings (cotton and vinyl-coated cotton/polyester blackout) were selected for this study. The experimental approach is to carry out full-scale room burns involving each of the eight combinations of double layers ignited with a simulated wastepaper basket. The same combinations will be evaluated in the NFPA 701 large-scale test apparatus and by the ASTM D 3659-80 semi-restraint method; the latter is being considered for inclusion in the NFPA 701 standard. The children's sleepwear apparatus, which is currently used for the NFPA 701 small-scale test, has been adapted for the semi-restraint method. At the time of writing, all of the test specimens have been prepared and testing is being initiated. Funding is being sought for extending this program to other fabric combinations and for multiple-track curtain and drapery systems.

\section{Publications:}

None to date; however, the major deliverable will be a test procedure for evaluating the fire behavior of multiple-layer fabrics for inclusion in the NFPA 701 standard. 


\title{
FIRE PERFORMANCE AND TOXICITY OF COMPOSITE MATERIALS
}

\author{
Funding Agency: Navy \\ Project Leader: Emil Braun \\ Project Objective:
}

To assist the Navy in developing fire safety performance requirements and evaluation procedures for use of composite materials aboard submarines.

\section{Technical Accomplishments:}

The scope of this work limited CFR involvement in the development of fire safety performance requirements and evaluation procedures to an analysis of the applicability of the NBS Toxicity Protocol and the Cone Calorimeter for composite material aboard submarines. Several candidate materials were supplied by the Navy and used in the evaluation of the test procedures and the acceptance criteria contained in the second draft of the military standard.

Tests were conducted using a modified NBS Toxicity Protocol as defined in the military standard. As a result of these tests, the Navy has been encouraged to adopt an N-Gas Toxicity Procedure as the basis for a first estimate of material toxic potency. Since such a procedure has never been fully developed, we are currently drafting such a document. This calls for a modification of the acceptance criterion for "super toxicity" as defined in the military standard for use of composite materials aboard submarines, draft 2 .

The same materials were tested for fire performance using the Cone Calorimeter. Once an acceptable fire scenario is developed by the Navy, suitable performance requirements can be established for this test method.

\section{Publications:}

Report of Test on the Fire and Toxicological Behavior of Four Materials (report in preparation).

Test Procedure for the Implementation of N-Gas Toxicity Protocol (report in preparation). 


\section{A TECHNICAL ASSESSMENT OF EXPLOSIVE VAPOR DETECTION DEVICES}

Funding Agency: Law Enforcement Standards Laboratory, National Institute of Standards and Technology

Project Leader: Marc R. Nyden

Project Objective:

Report on the present and future capabilities of explosive vapor detectors for the purpose of providing technical guidance to the representatives of local law enforcement agencies.

\section{Technical Accomplishments:}

The major findings of this investigation were as follows:

1) Portable explosive vapor detectors are capable of detecting a limited range of organonitrate explosives including ethylene glycol dinitrate, nitrotoulene, di-nitrotoulene, nitroglycerine, and, in some cases, trinitrotoulene.

2) Commercially available models are much less effective in detecting cyclonite, pentaerythritol tetranitrate, flammable liquids, and inorganic nitrates. The inability to detect the latter two categories of explosives is a serious limitation as they are frequently encountered explosive fillers.

3) Recent advances in the design of explosives detection devices have become manifest in the form of large, complicated, and expensive instruments which are most suitable for use as dedicated detectors at major installations such as military bases and international airports. Research efforts appear to be directed more at improving sensitivity than at increasing the field-worthiness and reducing the cost of these instruments. The possibility of improving search effectiveness by introducing computer technology has not been fully examined.

Reports and Publications:

"A Technical Assessment of Explosive Vapor Detection Devices," Marc R. Nyden, a report prepared for the National Institute of Justice (in preparation). 
CENTER FOR FIRE RESEARCH

OTHER AGENCY PROJECT - 1988

FIRE CONDITION EFFECTS ON SMOKE GENERATION AND TOXICITY

\section{Funding Agency: Industry Coalition}

Project Leader: Vyto Babrauskas

\section{Project Objectives:}

Measuring the generation rates of soot and the principal toxic gases as a function of ventilation, irradiance, fire size, and sample nature and orientation leading to:

- Sufficient knowledge for predicting full-scale smoke generation from small-scale data;

- Deriving a validated set of combustion conditions for toxicity measurement;

- Evaluating the usefulness of existing toxicity measurement devices as a means of obtaining input data for fire hazard assessment.

\section{Technical Accomplishments:}

Parallel instrumentation for measuring fire smoke yield and obscuration has been developed for both the Cone Calorimeter and the Furniture Calorimeter. For solid and liquid combustibles, it was found that large-scale smoke emission rates could be well represented from the Cone Calorimeter data after matching the specimen mass loss rates per unit area. This matching was accomplished in bench scale by exposing the specimens to a suitable level of external irradiance. The results were expressed in terms of the specific extinction area, which is defined as the smoke extinction cross-section per unit mass. The specific extinction area, when computed on a smoke particulate mass basis, was found to be independent of fuel type and of the conditions of test.

A first set of experiments has been performed to determine the sensitivity of smoke and toxic gas yields to the combustion conditions. Operating the Cone Calorimeter at a single irradiance, samples were combusted under various air flows and differing oxygen concentrations in the flow. Significant variation in the carbon monoxide yields was observed. The data are being analyzed further to direct the next set of experiments.

\section{Publications:}

Babrauskas, V., and Mulholland, G.W., "Smoke and Soot Data Determinations in the Cone Calorimeter," ASTM Symposium on Mathematical Modeling of Fires and Related Test Methods, New Orleans, December, 1986.

Mulholland, G.W., Henzel, V., and Babrauskas, V., "The Effect of Scale on Smoke Emission," Second International Symposium on Fire Safety Science, Tokyo, 1988 . 


\section{CENTER FOR FIRE RESEARCH \\ OTHER AGENCY PROJECT - 1988}

\section{HEAT FLUX SENSOR CALIBRATION}

Funding Agency: Federal Aviation Administration

Project Leader: Kenneth D. Steckler

Project Objective:

To evaluate the use of heat flux sensors in FAA fire tests and to establish a means for calibrating these sensors.

Technical Accomplishments:

This work focuses on the FAA proposed test method for aircraft cabin interior materials. This test method requires a measurement of the exposure (external) radiant heat flux incident upon the material or product to be tested. Thin foil (Gardon) heat flux sensors are used for this purpose.

A comparative (substitution) calibration technique is being developed. This approach entails identifying a well-characterized reference radiometer and a stable radiant source, constructing an apparatus for aligning the source and sensor, and assessing the accuracy and consistency of the calibration apparatus and procedure.

A self-calibrating electrically heated cavity radiometer (ECR) is being evaluated for use as a reference standard. The ECR contains a small blackened cavity which absorbs the radiative flux. This flux produces a measurable increase of the cavity's temperature. A resistance heater wound into the walls of the cavity and powered by a high precision power supply allows the injection of a precise amount of energy into the cavity for calibration purposes. The Radiometrics Physics Division of NIST has characterized the ECR with respect to absolute accuracy, linearity, and blackness. Overall accuracy was found to be within 2 percent up to $1 \mathrm{~W} / \mathrm{cm}^{2}$.

The calibration apparatus has been designed and is currently being fabricated. It consists of a 6 -inch diameter electric radiant heater capable of establishing fluxes in the range 1 to $4 \mathrm{~W} / \mathrm{cm}^{2}$ along its axis. Water-cooled baffles maintained at ambient temperature insure that a heat flux sensor positioned along the axis of and pointed at the heater "sees" only radiation from the heater and from a well-defined ambient environment.

In addition to calibrating a sensor at various flux levels, the effect of the spectral characteristics of the sensor's "black" surface will be assessed by changing the temperature of the source and the source-to-sensor distance so as to produce the same total incident radiative flux, but with different spectral content.

Publications: None 

PART II. Timely Response to Current Fire Problems (Projects Funded by Other Agencies and Private Sector Organizations)

B. SMOKE`MOVEMENT AND CONTROL 


\section{CENTER FOR FIRE RESEARCH \\ OTHER AGENCY PROJECT - 1988}

\section{FIRE GROWTH/SUPPRESSION WITHIN AIRCRAFI}

Funding Agency: Federal Aviation Adrinistration, DoT

\section{Project Leader: King-mon Tu}

\section{Project Objective:}

To evaluate and quantify the effects of changes in present aircraft ventilation patterns on passenger cabin environment during in-flight fires.

\section{Iechnical Accomplishments:}

Conditions resulting from a steady state fire located in the interior of an otherwise reasonably sealed, systematically ventilated enclosure were stucied regarding the effects of fire size, ventilation rate and location, and ventilation direction: either having the inlet at the ceiling with the exhaust at the floor or vice versa. Additionally, the effects on the fire environment of having vents open to the ambient, located near the ceiling at either end of the enclosure, were studied. For the situation with the inlet at the ceiling and over the range of interest the rate of ventilation had little effect on the gas, wall, and ceiling temperatures. These temperatures varied directly with fire size (ranged from 6 to $60 \mathrm{kH}$ ). Most of the energ: released by the fire was transferred to the ceiling above the fire. To first order, the combustion product's chemical species concentrations increased linearly with ventilation times and fire size. The introduction of small vents had little effect on the thermal environment but a substantial effect on cherical species concentration was noted. By reversing the flow direction, that is, having the fresh air iniet at the floor and the exhaust at the ceiling a substantial lowering of the environment temperature and species concentration in the interior of the enclosure was observed.

\section{Publications:}

Fire Environtent in Counterflow Ventilation (The In-Flight Aircraft Cabin Fire Problem.): B. McCaffrey and h. Rinkiner, National Bureau of Standards Interagency Report, NBSIR 88-3086, June 1988.

Effect of Venting Through Small Hatches Near the Ceiling on CounterflowVentilated Enclosure Fires, K. Tu, h. Rinkinen, B. McCeffrey, National Bureau of Standards Interagency Report, NBSIR 88-3834, July 1988.

Effect of Reversing Ventilation Direction on the Environment in ForcedVentilated Enclosure Fires, B. McCaffrey, K. Iu, h. Rinkinen, National Bureau of Standards Letter Report, August 1988.

\section{Related Grants:}

"Fire Environment In Counterflow Ventilation," Bernard McCaffrey, University of Maryland. 
CENTER FOR FIRE RESEARCH

NATIONAL BUREAU OF STANDARDS

FY88

Institution: The University of Maryland Baltimore County

Grant No.: 70NANB8H0819

Grant Title: Fire Environment In Counterflow Ventilation

Principal Investigator: Prof. Bernard J. McCaffrey

Department of Mechanical Engineering

University of Maryland Baltimore County

Baltimore, MD 21228

Other Professional Personnel: Scot P. Deal, Graduate

Research Assistant

NBS Scientific Officer: Dr. King-Mon Tu

\section{Technical Abstract:}

Introduction- The work described herein involves the study of the effects of forced ventilation on the fire environment in an enclosed volume. A unique aspect concerns the direction of the ventilation flow which is opposed to the buoyancy forces of the fire-generated hot gases, that is, fresh air is forced into the enclosure at the ceiling and exhausted at the floor of the otherwise sealed "box". The study is primarily experimental although a future analytical component is planned in an effort to generalize the experimental results. The findings of a previous NBS study using the same facility, Ref. rl, describe the complete thermal field in the enclosure as a function of fire size and ventilation rate. The conclusions indicated that the ventilation rate within ranges considered had little effect on the ability of the system to promptly remove energy generated by the fire. The ventilation did little to prevent the buoyancy forces from tending to keep the enclosure quite stratified. Most of the heat released by the fire was absorbed by the ceiling above the fire. In this work the chemical species concentrations in steady state at various positions throughout the enclosure will be described for the present ventilation configuration and range of ventilation flow rates. We hope to gain some insight into the large scale eddy mixing associated with this kind of strongly buoyant, weakly forced-ventilated environment. Future studies of changes in ventilation patterns, rates, etc. will be compared to these baseline results. Additionally, a study aimed at elucidating the near-wall or confinement phenomena associated with diffusion flames is taking place concurrently.

Experimental- The enclosure is $4.8 \times 2.4 \times 1.2 \mathrm{~m}$ high, made of galvanized sheet and containing a ceiling and floor plenum constructed of calcium silicate board (marinite) which allowed for two separate pairs of air inlet configuration positions in the ceiling and a single pair for exhaust in the floor. The inlet and outlet slits run the full length of the longer dimension of the box. (See Fig. 1) For the experiments described here 32 simulated 
seats of aluminum and marinite were symmetrically arranged in the box. The fire, a constant feed of propane through a diffusive burner at the floor, was located in the geometric center of the enclosure and exhibited heat release rates between 10 and $60 \mathrm{~kW}$. Ventilation flow rates varied between two and six minutes for one enclosure volume exchange. Approximately 60 channels of data were recorded in time during each test. Complete details of this facility are contained in $[r l]$.

Results-Fig. 2 shows a scatter plot of all the data for normalized $\mathrm{CO}_{2}$ $(+)$ and $\mathrm{O}_{2}(\square)$ gaseous concentration for five positions in the enclosure and also for an additional $\mathrm{O}_{2}(\Delta)$ sample in the exhaust gas. Positions near the ceiling exhibit higher concentrations, data from lower points in the box are similar to values in the exhaust. The abscissa is a non-dimensional generation to ventilation rates ratio and the straight line shows a calculation of what a well stirred reactor would exhibit. These are steady state values and represent concentration averages of the data from $450 \mathrm{~s}$ to 800s. A typical transient plot can be observed on Fig. 3.

Assuming complete combustion of propane, the reaction with excess dry air is illustrated in the following:

$$
\mathrm{C}_{3} \mathrm{H}_{8}+5(1+\mathrm{x})\left[\mathrm{O}_{2}+3.76 \mathrm{~N}_{2}\right]=3 \mathrm{CO}_{2}+4 \mathrm{H}_{2} \mathrm{O}+5 \mathrm{xO}_{2}+5(1+\mathrm{x}) 3.76 \mathrm{~N}_{2}
$$

The mole fraction of $\mathrm{CO}_{2}$ in the exhaust is $\left[\mathrm{CO}_{2}\right]=3 /(25.8+23.8 \mathrm{x})$

The mole fraction of $\mathrm{O}_{2}$ in the exhaust is $\left[\mathrm{O}_{2}\right]=5 \mathrm{x} /(25.8+23.8 \mathrm{x})$

Or if it is assumed that at the instant before introduction of $\mathrm{C}_{3} \mathrm{H}_{8}$ that the mole fraction of $\mathrm{O}_{2}$ is 0.21 then,

$$
0.21-\left[\mathrm{O}_{2}\right]=5.42 /(25.8+23.8 \mathrm{x}) \text { and hence, }
$$

the Normalized Concentration is defined as:

$$
\left[\mathrm{CO}_{2}\right] / 3=\left(0.21-\left[\mathrm{O}_{2}\right]\right) / 5.42=1 /(25.8+23.8 \mathrm{x})
$$

This is the method for plotting the data in Fig. 2 and provides a consistency check since these are results from two independent instruments. Note the small amount of $\mathrm{CO}$ and soot present in these experiments hardly affects the accuracy of the equality at all. The extent of agreement can be observed in Fig. 2 by comparing any pair of data sets. For clarity in subsequent plotting and analysis we can observe the average of the two as representative of that particular point.

Through a similar analysis the right hand side of the above can be evaluated in terms of heat release rate of the fire (the generation of the chemical species) and the ventilation rate (the dilution). In the absence of sources and sinks, after the transient, the average for the box or the well stirred reactor result is approximately equal to the volume flow rate of chemical species to that of the ventilation flow

$$
\frac{\mathrm{O} / \mathrm{H}}{\dot{\mathrm{V}}}=\frac{\mathrm{O}(\mathrm{kW}) /(46343 * 1.92)(\mathrm{kJ} / \mathrm{m} * \mathrm{~m} * \mathrm{~m})}{14.5 \mathrm{~m} * \mathrm{~m} * \mathrm{~m} / 60 / \mathrm{t}(\mathrm{min})}=4.7 * \mathrm{E}-5 \mathrm{Q}(\mathrm{kw}) * \mathrm{t}(\mathrm{min})
$$

where $Q$ is the heat release rate of the fire and $t$ is the time for one volume exhaust. In Ref. r2 Pagni, et al. formalized and generalized the above including the transient portions.

Figure 4 contains all the date seen in Fig. 2 showing the vertical distribution in the box and plotted alongside a steady state temperature profile for TC tree "C". From the results of Fig. 2 we can factor out first order effects of fire size and ventilation rate by dividing the Norm. Concentration by the right hand side of the above equation. The scale then 
varies about the value of 1 , the well stirred result. The filled symbols are results for the "wall" ventilation configuration (Fig. 1) and the others correspond to the "central" configuration. In general the "wall" yields higher concentrations for the centerline positions shown in the figure. Whether it is due to a simple local increase in dilution since the source of the fresh air is closer to the sampling position (the cold air in the wall case could travel down adjacent to the wall and exit at the floor) or to a more complex flow pattern is not clear from Fig. 4. The data symbols represent averages of the data at the particular position; the bars represent high and low values and the numbers below the symbols is the number of data sets. In general the lower end of the bar corresponds to longer ventilation times and the upper end of the range corresponds to higher heat release experiments. These second order effects then would dictate steady state concentrations varying with $Q$ to somewhat greater than linear and the variation with ventilation time to a somewhat less than a linear relation. Again one might speculate that with lower flow velocities in the longer time cases there is less penetration through the hot upper layers yielding more dilution.

Preliminary results indicate that for $7.5 \times 15 \mathrm{~cm}$ holes placed at the top center of the two end walls a $7 \%$ reduction at the top position and a $16 \%$ reduction in exhaust concentration could be attained. For $15 \times 15 \mathrm{~cm}$ holes the reductions increase to 168 at the top and 248 in the exhaust. For hole size double again reductions increase to 228 and 408 respectively.

\section{References -}

rl. McCaffrey, B. and Rinkinen, W., Fire Environment In Counterflow Ventilation (The In-Flight Aircraft Cabin Fire Problem) NBSIR 88- (in preparation)

r2. Pagni, P., Alvares, N., and Foote, K., Defining Characteristic Times in Forced Ventilation Enclosure Fires, American Society for Testing and Materials, STP 983 (1988).

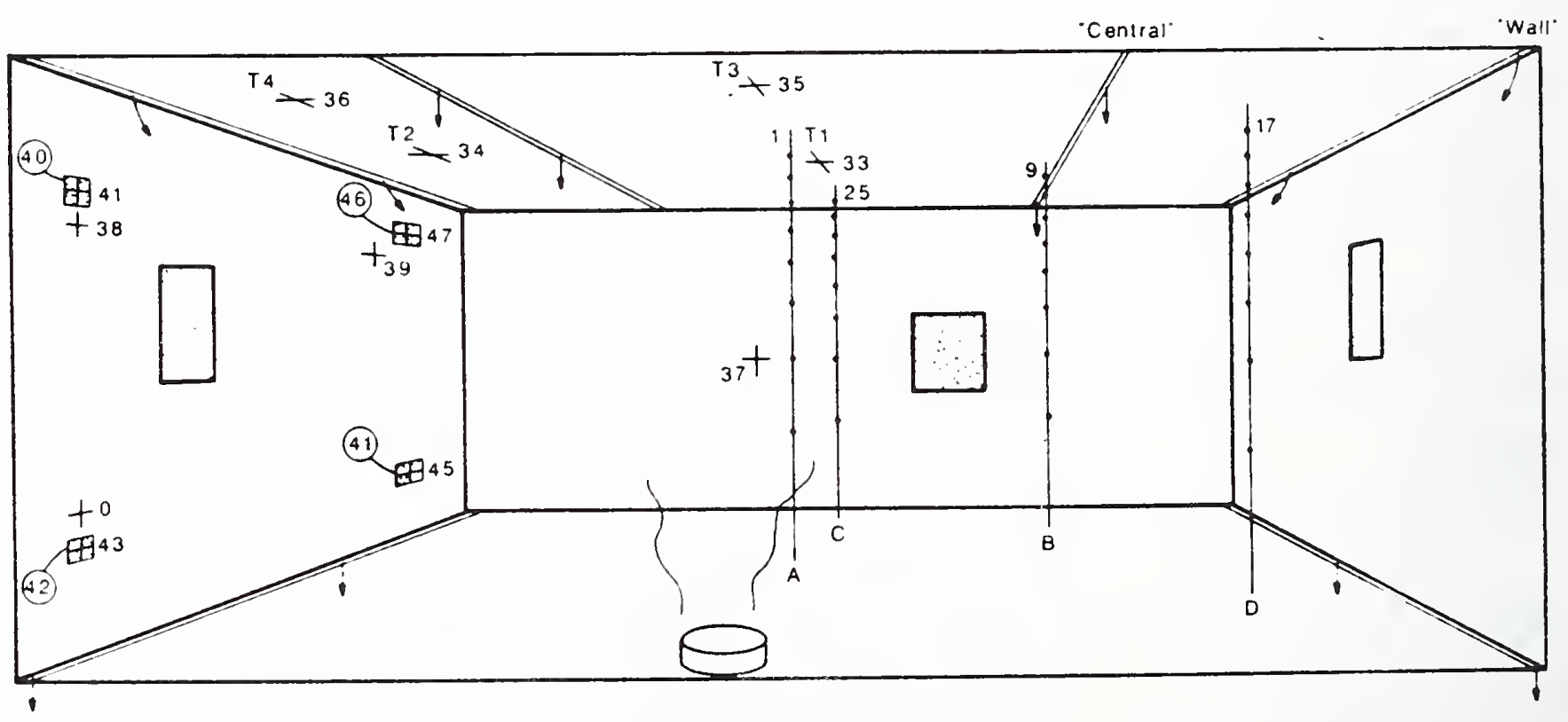

Fig. 1: INTERIOR VIEW OF ONE-HALF OF SYMMETRIC ENCLOSURE (SEATS NOT SHOWN) 


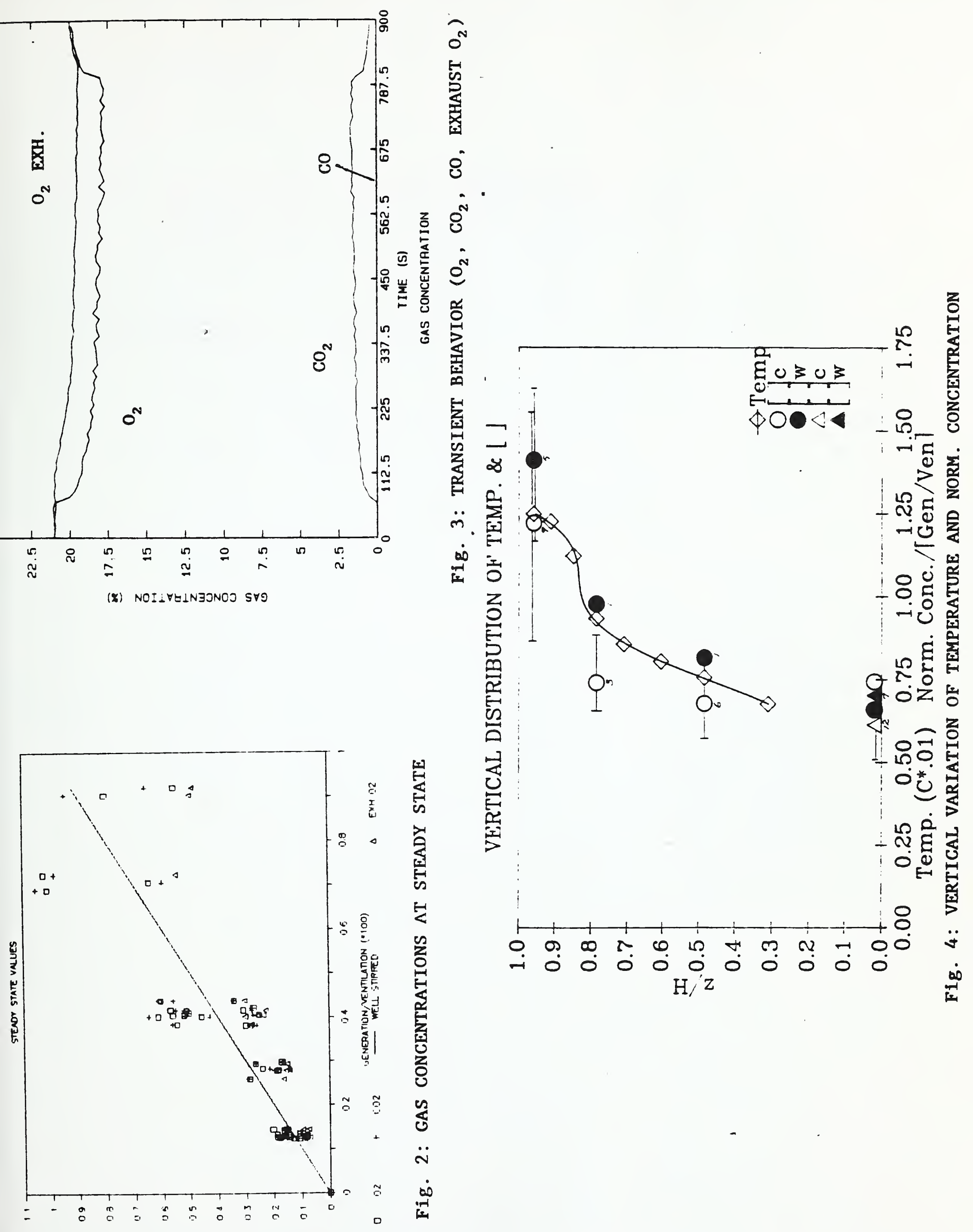


PART II. Timely Response to Current Fire Problems

(Projects Funded by Other Agencies and Private Sector Organizations)

C. FIRE PROTECTION TECHNOLOGY 



\section{CENTER FOR FIRE RESEARCH \\ OTHER AGENCY PROJECT - 1988 \\ FIRE AND THERMAL CHARACTERISTICS OF NAVY FIRE FIGHTING TRAINERS}

Funding Agency: Navy Training Systems Center, Orlando, Florida

Project leader: $R$. S. Levine

Project Objective:

Support the development and implementation of prototype fire fighter trainers at Mayport Naval Station, Jacksonville, Florida.

\section{Technical Accomplishments:}

Using fire compartment models, wall and gas temperatures, radiant fluxes, oxygen concentrations, and ceiling layer heights were predicted for the trainer compartments with large fires in them. Measurements will be made during Navy acceptance tests of these trainers, and the results used to validate or improve the models as design tools for the sponsor and for related CFR use.

In addition, the project leader has acted as a consultant on safety and other concerns on the pre-prototype trainer at the Norfolk, Virginia Navy Fire Training School.

\section{Reports and Publications:}

Most results to date are incorporated in project letters to the Naval Training Systems Center.

NBSIR 88-3755, Stroup, D. 'T., Naval Fire Fighting Trainers-Thermal Radiation Effects Associated with the $19 \mathrm{~F} 4 \mathrm{FFT}$. 


\section{CENTER FOR FIRE RESEARCH \\ OTHER AGENCY PROJECT - 1988}

\section{FALSE ALARMS FROM SMOKE DETECTORS}

Funding Agency Veterans Administration and U.S. Air Force

Project Leader Richard W. Bukowski

Project Objective

To conduct a field study of false alarms produced by smoke detectors installed within VA Medical Centers to identify causes and mitigation strategies.

\section{Technical Accomplishments}

In 1987, a survey of 133 VA Medical Centers (VAMC), out of a total of 172 throughout the U.S., coupled with visits to 20 facilities, was conducted to gather data on false alarms of smoke detectors.

Information requested for the study included name of the detector manufacturer and model number, control unit manufacturer and model number, number and type of detectors installed, where installed, number of false and real alarms for preceding year, date of installation, and policies on smoking, testing, cleaning, and maintenance. VAMC personnel involved with the installations were requested to indicate the maximum level of false alarms that could be tolerated and to provide any recommendations to reduce their occurrence.

The study included a total of approximately 37,000 system type smoke detectors of which 69 percent were of ionization (ion) type and 31 percent photoelectric, 3000 duct detectors (90 percent ion and 10 percent photo), and 1100 smoke detector modules ( 80 percent ion and 20 percent photo) integral with door holder closers (DHC). Also, included are approximately 100 single station smoke alarms.

In 1988 , a $(\approx 250$ page) report of the study was produced, encompassing the following subject areas:

1. Background information which includes a history of smoke detectors from prior to World War II up to the present as well as the roles played by evaluation and installation standards.

2. Technical background information describing: (a) principle of operation and susceptibility to false alarms resulting from their use, (b) method of measurement of the detector sensitivity (threshold response), and (c) production window.

3. Review of previously-conducted false alarm studies.

4. Analysis of the various causes of false alarms.

5. Effect of sensitivity, environmental factors, misapplication, smoking, lack of testing and cleaning, and other miscellaneous VAMC operational procedures on false alarm rates. 
6. Discussion of the various testing procedures employed at the VAMCS.

7. Suggested short-term remedies for presently installed detectors including use of alarm verification, presignal operation, detector replacement, cross zoning, and the like. Long term remedies are also included through changes in product performance and installation standards.

8. Recommendations for reducing false alarms are included for: the VAMCS, VA Central office, testing agencies, NFPA 72E committee, detector manufacturers, system designers, and industry associations. A number of these recommendations have been presented to the appropriate organizations, and are being adopted.

9. Evaluation of sensitivity tests conducted on detector samples removed from VAMC installations that were visited.

10. Discussion of the shared responsibility of the various parties involved with smoke detectors including the design, manufacture, evaluation, installation, and inspection by the local authority having jurisdiction.

11. Numerous tables are included documenting false alarm prevalence by; VAMC, detector manufacturer, detector model, type of detector, principle of operation, frequency of cleaning and testing, location, sensitivity, and the like.

12. Attachment of a practical false alarm goal, design of future detectors, and remaining activities.

The report is written as a reference document for the VAMC staff involved with the fire alarm system on a daily basis. It will hopefully provide them with the information necessary to minimize false alarms from their systems while not sacrificing system performance. The draft document has been reviewed by VA Headquarters staff, and praised for its comprehensive treatment of the subject. 
CENTER FOR FIRE RESEARCH

OTHER AGENCY PROJECT - 1988

SAFETY IN OFFSHORE DRILLING

Funding Agency: Minerals Management Service, DoI

Project Leader: David Evans

Project Objective:

Examine technologies that can mitigate the effects of fire and oil spills from offshore platform accidents.

\section{Technical Accomplishments:}

Measurements of the effects of water spray on methane jet flames that simulate gas-well blowout fires were continued and expanded to include mixed methaneheptane flames. Through a grant to Professor Gore at the University of Maryland, the laminar flamelet concept for analysis of turbulent flames developed by Professor Faeth with CFR Grants Program support, is being used by Professor Gore to predict jet-flame structure and emitted radiation. Previous analysis of methane flames has been extended to methane-water flows and investigations of flame extinction.

Experiments completed two years ago by Professor Bourgoyne at Louisiana State University were reported. This report documents extension of natural gas jetflame water spray extinction conditions, previously measured by CFR, to energy release rates of $400 \mathrm{MW}$. For these large flames the water to natural gas mass flow rate ratio of 2.0 produces flame extinction when the water is sprayed along the edge of the vertical flame envelope. Measurements on obstructed flames showed that the ratio for extinction is 15.0 or greater.

Measurements of crude oil spill pool fire combustion energy release rates and smoke production were completed. Polycyclic Aromatic Hydrocarbon content of the smoke was found to be equal or less than that in the original oil.

\section{Publications:}

An experimental study of suppression of obstructed gas well blowout fires using water sprays, M. Chauvin and A. Bourgoyne, National Bureau of Standards Government Contract Report, NBS-GCR-88-547, June, 1988.

Environment Effects of Oil Spill Combustion, D. Evans, G. Mulholland, D. Gross, H. Baum and K. Saito, National Institute of Standards and Technology Interagency Report, NISTIR 88-3822, September 1988.

\section{Related Grants:}

"An Investigation of Simulated Oil Well Blowout Fires," Jayavant P. Gore, University of Maryland.

"A Study of Oil-Slick Combustion," Koso Saito and Robert A. Altenkirch, University of Kentucky. 
Institution: The University of Kentucky

Grant No.: $\quad$ 6ONANB7D0739

Grant Title: A Study of Oil-Slick Combustion

Principal Investigator: Prof. Kozo Saito

Department of Mechanical Engineering

University of Kentucky

Lexington, KY 40506-0046

Other Professional Personnel: Prof. Robert A. Altenkirch

Dr. Masataka Arai

Visiting Scientist

Sandra K. Elam

Graduate Research Assistant

NBS Scientific Officer: Dr. David D. Evans

Technical Abstract:

Introduction Crude oil is produced in many places in the world, and accidental spills in oceans of ten cause environmental problems. Combustion is a possible useful method of removing a crude-oil slick from an ocean surface. To develop an understanding of the fundamental combustion behavior of crude oil, scale model tests and numerical model calculations have been carried out by several investigators. From the results of these studies, the combustion rate of crude oil can be estimated, but they do not allow the combustion behavior of a crude-oil slick on water to be described accurately. For example, under certain circumstances, the water on which a burning pool of crude oil is supported may begin to boil. However, no model calculations that include the boiling phenomenon of the water exist. Here, we present a method for predicting the thermal conductivity of crude oil and experimental results that describe the boiling phenomenon of the water sublayer. These results can be used to guide the development of calculational models for oil-slick combustion.

Prediction of Thermal Conductivity The thermal conductivity of Alberta Sweet, La Rosa, and Murban crude oils was measured using the transient hot-wire method. In this method, the thermal conductivity of the liquid is determined by comparing the measured time dependent temperature change of a wire through which a current is passed that is submerged in a reservoir of the liquid at known initial temperature with the known analytical solution to the transient conduction problem. The measured thermal conductivity, $\lambda_{m}$, for the three crude

oils over the temperature range 0 to about $50{ }^{\circ} \mathrm{C}$ is shown in Fig. 1 . By considering the oils to consist of a mixture of aromatic and paraffinic fractions, the $\lambda_{m}$ were to $f$ it to the equation 


$$
\lambda_{m}=\lambda_{a}^{\omega_{a}} \lambda_{p}^{\omega_{p}}
$$

in order to determine $\lambda_{a}$ and $\lambda_{p}$. In Eqn. (1), subscripts a and $p$ imply the aromatic and paraffinic fraction, respectively, and $\omega$ is the mass fraction of the indicated functional group normalized such that $\left(\omega_{a}+\omega_{p}\right)=1.0$. The values of $\lambda_{a}$ and $\lambda_{p}$ determined experimentally then can be used to estimate $\lambda_{m}$ values for a crude oil if the composition of the oil is known. $\lambda_{a}$ and $\lambda_{p}$ do not vary much over the temperature range of the measurement, their average values being 215 and $108 \mathrm{~mW} / \mathrm{m} \cdot \mathrm{K}$, respectively.

Combustion Behavior of Oil Floating on Water: A schematic of the typical sequence of events of the combustion phenomenon is shown in Figs. 2 (a) through (e). Boilover of the water sublayer, step (c), occurs for fuels whose boiling point temperature is higher than that of water regardless of whether the fuel is pure or is a multicomponent fuel. The intensity of the boilover depends on the boiling point temperature of the fuel; the higher the boiling temperature of the fuel, the more intense is the boilover. Multicomponent fuels of course do not have a unique boiling point temperature, so we expect the intensity of the boilover to depend on the molecular weight or density of the fuel. The heavier the fuel, the higher the boiling point range and the more intense the boilover will be.

For pure fuels, the sequence of events depicted in (a): the onset of boiling. (b): normal boiling, (d): the end of boiling, and (e): extinction is common, except for the fact that high boiling point fuels, e.g., n-decane, occasionally behave as shown in step (c): appearance of a fireball-like flame. For multicomponent fuels and crude oils, the entire sequence of events (a) through (e) is common. When step (c) occurs, the fireball-like flame emits intense radiation to the surroundings. The size of the fireball-like flame depends on the intensity of the boilover and the fuel type. In an extreme case, its size is several times larger than the diameter of the pan.

Onset of Water Boiling In Fig. 3, a peak temperature in a burning fuel layer, $T_{p}$, is plotted against the time, $t_{p}$, required to reach $T_{p}$. For fuels with a boiling point temperature, $T_{b p, f}$, less than that of water, $T_{b p, w} T_{p}$ is the maximum temperature of the thermocouple near the fuel-water interface. If boilover occurs, which happens when $T_{b p, f} \geq T_{b p, w}$, then $T_{p}$ is the temperature indicated by the thermocouple just prior to when boilover begins. Numbers written next to the data points in Fig. 3 indicate $T_{b p, f}$.

For paraffins, $T_{p}$ and $t_{p}$ increase with increasing $T_{b p, f}$, and $T_{p}$ is nearly $T_{b p, f}$ until $T_{b p, f}$ is approximately equal to $T_{b p, w}$. Once $T_{b p, f}>T_{b p, w}$. $T_{p}$ is not much greater than $T_{b p, w}$, and $t_{p}$ decreases with increasing $T_{b p, f} \cdot$ This situation arises because when $T_{b p, f}<T_{b p, w}$ the water has little influence on the combustion process. When $T_{b p, f}>T_{b p, w}$, the water affects the combustion process. Just after ignition, the fuel surface temperature rapidly approaches 
$T_{b p, f}$, resulting in in-depth heating of the fuel. But the temperature of the fuel near the fuel-water interface cannot substantially exceed $T_{b p, w}$ because boiling of the water ensues. The amount of time that it takes the fuel near the fuel-water interface to reach approximately $\mathrm{T}_{\mathrm{bp}, \mathrm{w}}$ decreases with increasing $T_{b p, f}$ because the temperature gradient and heat transfer rate into the fuel layer increases as the surface temperature increases. Multicomponent fuels behave somewhat differently than either paraffins or aromatics, and this is due to the fact that the properties of the fuel change during the progress of combustion as the lighter fractions boil off, and the heavier fractions remain.

Temperature measurements at the onset of boiling for multicomponent fuels are shown in Fig. 4. A fixed amount of superheat, $10 \mathrm{~K}$, for the liquid water is obtained, and $T_{p}$ for these fuels seems to correlate with the fuel density as we anticipated. The level that $T_{p}$ can reach is limited by the occurrence of boilover as discussed for pure fuels in conjunction with Fig. 3 . Although a unique boiling point for multicomponent fuels cannot be specified, the temperature range over which boiling occurs is higher than the boiling point for water, and so we obtain the behavior shown in Fig. 4.

Summary An experimental investigation of the boilover phenomenon for liquid fuel burning above a water sublayer indicates that boilover occurs when the boiling point temperature of a pure fuel is more than that of the water. In this situation, the fuel temperature near the fuel-water interface is somewhat limited by the presence of the boiling water and cannot reach the fuel boiling point. The boilover behavior of multicomponent fuels is similar to that of pure fuels except that for multicomponent fuels, after the onset of boilover, a large, fireball-like flame frequently appears. A thermal conductivity prediction method for crude oil was also developed by considering the oil to be composed of an aromatic and a paraffinic fraction and fitting measurements to determine the thermal conductivities of these fractions.

\section{Reports and Papers:}

1. S.K. Elam, I. Tokura, K. Saito, R.A. Altenkirch, "Thermal Conductivity of Crude Oils," submitted to Experimental Thermal and Fluid Science.

2. I. Tokura, K. Saito, R.A. Altenkirch, D.D. Evans, "A Study of Crude Oil Combustion: Thermal Conductivity of the Condensed Phase," presented at the Central States Section of the Combustion Institute meeting, 1987.

3. S.K. Elam, I. Tokura, K. Saito, R.A. Altenkirch, "Prediction of Thermal Conductivity of Crude Oil for use in Oil-Slick Combustion Modelling," presented at the Central States Section of the Combustion Institute meeting, 1988.

4. M. Arai, K. Saito, R.A. Altenkirch, D.D. Evans, "A Study of the Boilover Phenomenon for Liquid Fuel Supported on Water," presented at the Central States Section of the Combustion Institute meeting, 1988.

5. M. Arai, K. Saito, R.A. Altenkirch, "Experimental Study of Burning Liquid Fuels on a Water Sublayer," submitted for presentation in a poster session at the Twenty-Second International Symposium on Combustion, 1988. 

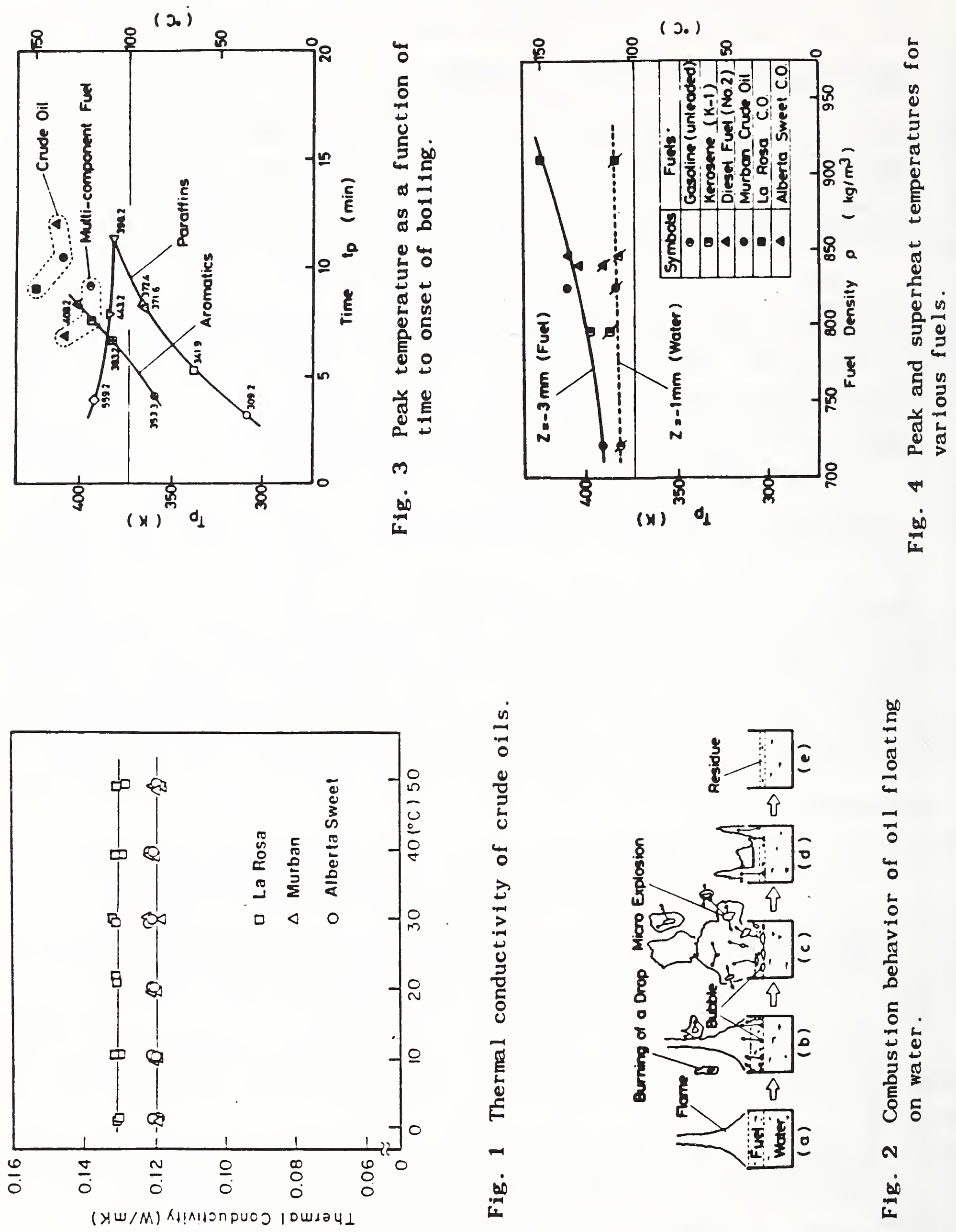


\section{CENTER FOR FIRE RESEARCH \\ NATIONAL INSTITUTE OF SCIENCE AND TECHNOLOGY \\ FY 88}

Institution: The University of Maryland at College Park

Grant No.: $\quad$ 60NANB8D0834

Grant Title: An Investigation of Simulated Oil Well Blowout Fires

Principal Investigator: Dr. J. P. Gore

Mechanical Engineering Department

University of Maryland

College Park, MD 20742

Other Professional Personnel: Steve Skinner, Undergraduate Assistant

NIST Scientific Officer: Dr. David D. Evans

Technical Abstract:

Introduction Radiation and extinction of large turbulent jet flames is of interest in the evaluation of hazard to personnel resulting from oil and gas well blowouts. Motivated by this problem, experiments concerning the feasibility of extinguishment of large (100-200 MW) and medium (1-10 MW) scale methane/air flames using water-sprays have been conducted in the past [r1,r2]. The objectives of the study reported here are: (1) to utilize data from reference [r2] for medium (1-10 MW) scale flames to evaluate the effectiveness existing analysis[r3-r5], and (2) to extend the analysis to treat two phase flow effects resulting from combined burning of heptane(to simulate oil) and methane(to simulate natural gas) and also liquid water suppressant. Effects of liquid water suppressant were considered during the first phase.

Experimental Data Existing experimental data[r2] were utilized during the initial phase of the project. Vertical jet flames burning methane at heat release rates between 1-8 MW were considered in reference [r2]. Six chrome1alumel thermocouple junctions formed from $0.5 \mathrm{~mm}$ wires were used to measure temperatures along the flame axis. Radiative heat fluxes to two representative locations around the flames (see inset of Fig. 2) were monitored using wide angle radiometers. All the flames considered were turbulent ( $R e-30,000$ to $500,000)$ and had some effects of buoyancy(Ri - 0.000014 to 0.00012 ) as per the criterion of reference [r6]. Many of the flames were lifted from the burner exit.

Theoretical Methods Theoretical methods for flames without the addition of water were identical to those of Jeng et al. [r3]. For flames with water added to the fuel stream, the locally homogeneous flow (LHF) approximation described in ref. [r7] was used. Within this approximation, it was assumed that the transport of mass, momentum, and energy between the liquid water and the surrounding gases is much faster than the mixing between the jet and the ambient fluids. Based on past experimental findings [r8], it was assumed that the addition of liquid water produced only thermal effects. These were treated by modifying the state relationships for all scalar properties as a function of mixture fraction. The structure calculations were completed using 
a Favre-averaged $\mathrm{K}-\epsilon-\mathrm{g}$ model similar to past practice using the GENMIX algorithm [r9]. Although, many of the flames were lifted from the burner, the effects of liftoff were neglected due to considerable uncertainties in the current understanding [r10]. The radiation calculations were performed using the RADCAL algorithm [r11] and the discrete transfer method [r12].

Results and Discussion Measurements (represented by symbols showing the best fit to the data from ref.[r2] and not actual data points) and predictions of temperature along the axis of a $4.6 \mathrm{MW}$ flame without water addition are shown in Figure 1. The measurements have not been corrected for the effects of radiation (expected to be ca. $200 \mathrm{~K}$ in the hottest portions). In regions near the burner exit the predictions mimic the data. This may be fortuitous in view of the lack of radiation corrections. In regions away from the burner, the measurements are $\mathrm{ca} .400 \mathrm{~K}$ lower than the prediction. This is probably because of the uncertainities of the analysis concerning initial conditions and flame liftoff.

Figure 2 shows measurements and predictions of total heat fluxes incident upon two detectors (for positions indicated in the inset) as a function of heat release rate. The shape, size, and position of the flame relative to the detectors change due to the effects of buoyancy [r6]. The data show that the heat flux to both detectors increases rapidly with heat release rate in the 0.5 to $2.5 \mathrm{MW}$ range, and then, due to the changing shape and size of the flame relative to the detector positions, the rate of increase is reduced considerably before increasing again at ca. $4.5 \mathrm{MW}$. It is encouraging to note that the analysis mimics this behavior. Based on the small differences in the predictions (which completely neglect radiation from soot) and measurements, it can be concluded that soot-radiation is a minor fraction of the total radiation for methane/air flames. The agreement between measurements and predictions of radiative heat fluxes is much better than those of temperatures. Part of this is due to the integral nature of radiative heat flux, resulting in cancellation of errors in the detailed scalar distributions. Based on the encouraging predictions for flames without wateraddition, the analysis was extended to treat the effects of water added to the fuel.

Figure 3 shows the state relationships for flames with water added to the fuel stream. Temperature, mole fraction of liquid water, mole fraction of water vapor, and mole fraction of carbon dioxide are plotted as a function of fuel equivalence ratio. Liquid water is present on the fuel-rich side of all the flames with water addition and holds the temperature at the equilibrium value corresponding to the partial pressure of water vapor. Once all the water is evaporated, the temperature rises rapidly, but its peak is lower due to the heat of vaporization and sensible heating of the water. Mole fractions of gases like carbon dioxide are reduced as shown in Figure 3 while mole fractions of water vapor are increased, resulting in non-trivial effects on absorption coefficients. As seen from the state relationships in Figure 3 , the present analysis implies that all the liquid water is evaporated within the flame. In practice, due to poor atomization particularly at large waterloadings, water penetrates into the fuel-lean regions and also gets ejected from the flame. This is expected to affect the temperature and radiation predictions for flames with water.

Measurements and prediction of peak temperatures along the axis of 4.6 MW flames as a function of nondimensional water mass flow rate are plotted 
in Figure 4. For a fixed methane flow (fixed heat-release rate), the location of the peak temperature (both measured and predicted) remains almost independent of the water flow rate due to the effects of increased momentum of the jet. As expected both data and predictions show a reduction in the peak temperature with increasing water-addition. Discrepancies between data and predictions for zero water-loading have been discussed above. As waterloading is increased, the differences between data and predictions are seen to decrease. This is not only because of the reductions in radiation corrections but also because of the assumption of complete water-evaporation. It is therefore expected that the radiative heat fluxes for flames with large quantities of water will be under-predicted by the analysis. Figure 5 shows measurements and predictions of radiative heat flux to a representative detector (normalized by the flux with zero water-loading) as a function of non-dimensional water flow-rate. As expected, the predictions under-estimate the heat flux for high water-loadings. Consideration of finite evaporation rates will be necessary for treating flames with the high water-loadings which are required for extinction.

Acknowledgement Part of this work is supported by the Mineral Management Service, Department of the Interior.

\section{$\underline{\text { References }}$}

[rl] Evans, D. and Pfenning, D., Oil and Gas J., 83 (17), 80, 1985.

[r2] McCaffrey, B. J., "Momentum Diffusion Flame Characteristics and the Effects of Water Spray," NBSIR 86-3442., NIST, Gaithersburg, MD, 20899, 1986.

[r3] Jeng, S. M., Lai, M. C., and Faeth, G. M., Combust. Sci. and Tech., 40, 41. 1984.

[r4] Jeng, S. M. and Faeth, G. M., J. Heat Trans. 106, 891, 1984.

[r5] Gore, J. P., Faeth, G. M., Evans, D. and Pfenning, D., Fire and Materials 10, 161, 1986.

[r6] Becker, H. and Liang D., Comb. Flame, 32, 115, 1978.

[r7] Faeth, G. M., Prog. Energy Combust. Sci., 9, 1, 1983.

[r8] Seshadri, K, Comb. Flame, 33, 197, 1978.

[r9] Spalding, D. B., GENMIX: A General Computer Program for TwoDimensional Parabolic Phenomena, Pergamon, Oxford, 1977.

[r10] Pitts, W., 1988, "Assesment of Theories for the Behavior and Blowout of Lifted Turbulent Jet Diffusion Flames," Twenty-Second Symposium

(International) on Combustion, The Combustion Institute, Pittsburgh, in press, 1988.

[r11] Grosshandler, W., Int. J. Heat Mass Transfer 23, 1447, 1980.

[r12] Lockwood, F. C. and Shah, N. B., Eighteenth Symposium

(International) on Combustion, The Combustion Institute, Pittsburgh, $\mathrm{Pa}$ 1405,1981 .

\section{Reports and Papers}

1. J. P. Gore, D. D. Evans and B. J. Mccaffrey, "Temperature and Radiation of Large Methane/Air Jet Flames with Water Supression, "Fall Technical Meeting of the Eastern Section of the Combustion Meeting, Stillwater, FL, submitted.

2. J. P. Gore and D. D. Evans, "Radiation from Two Phase Fires burning Heptane and Methane," ASME National Heat Transfer Conference 1989, Philadelphia, PA, submitted. 


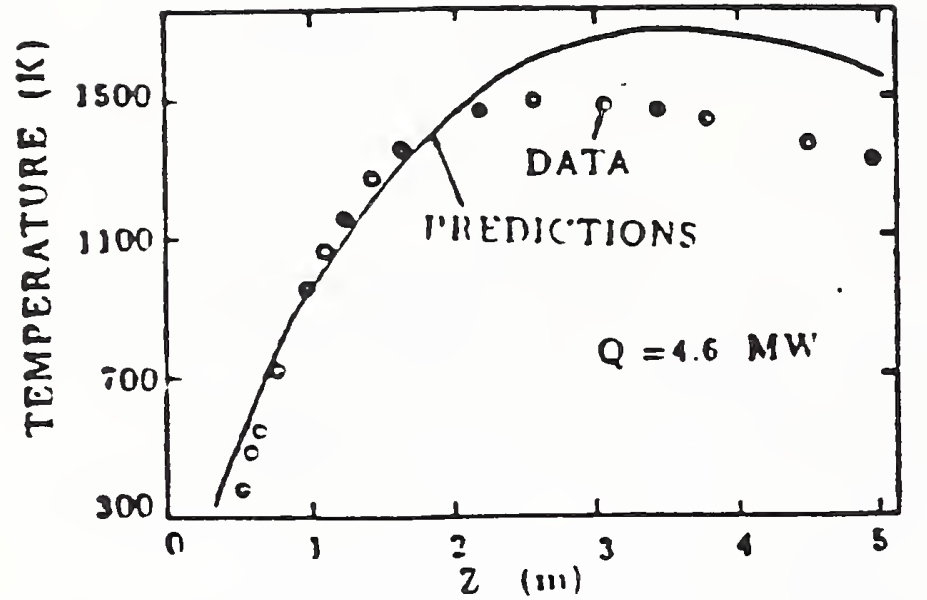

Fig. I Temperature along Flame Axis

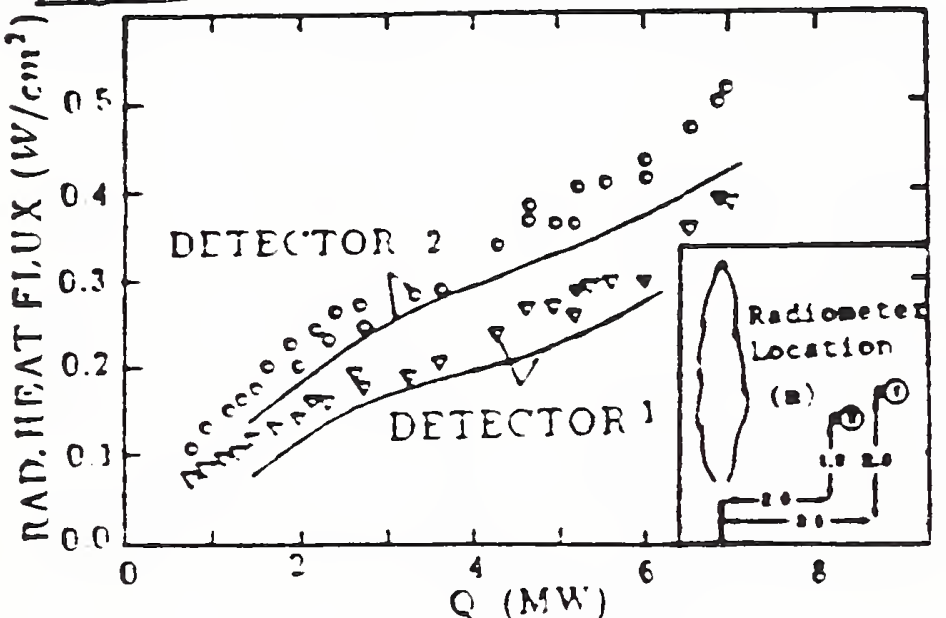

Fig. 2 Raciative Heat flues

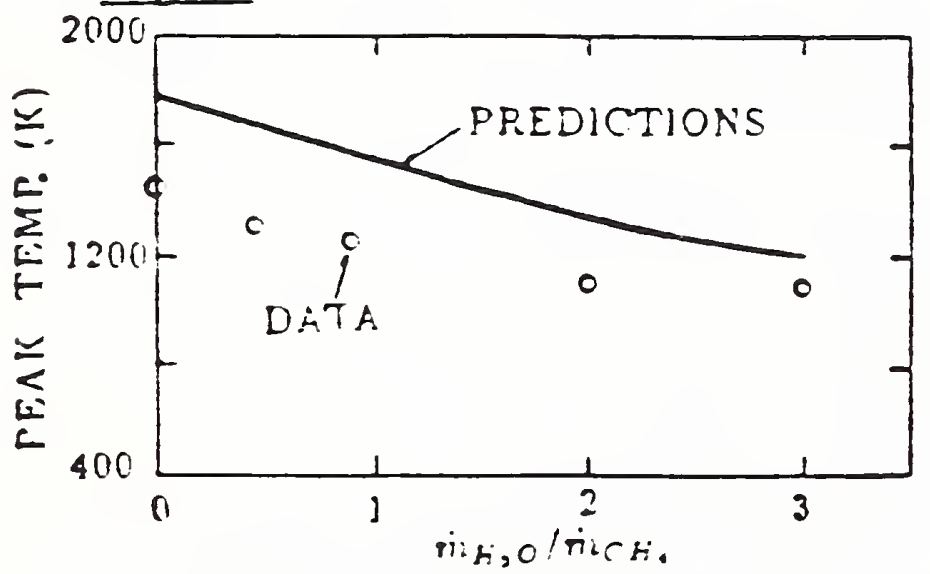

Fig. L PEak IeIpezatuzes with $\mathrm{H}_{2} \mathrm{O}$

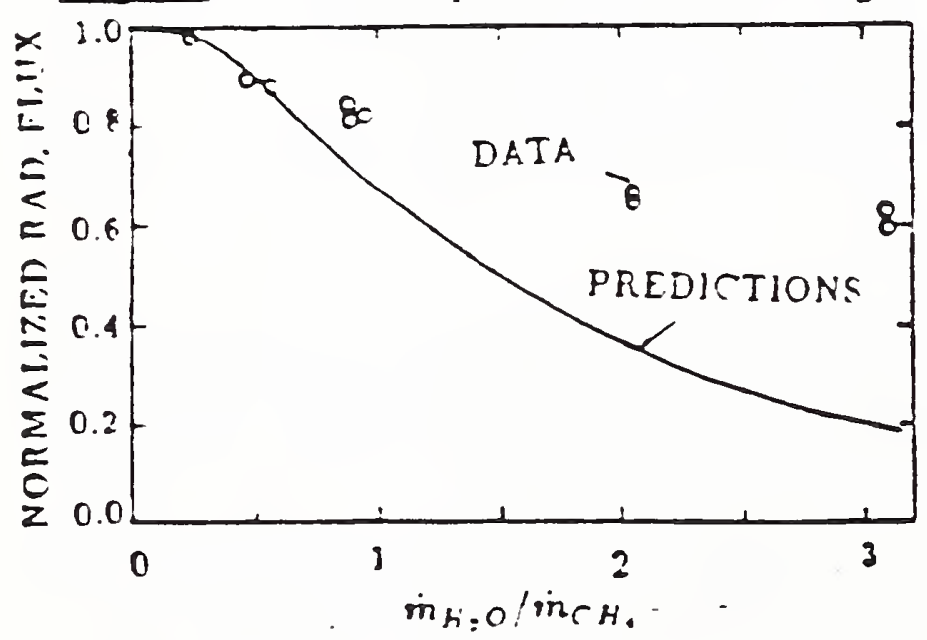

Fig. 5 Radiative Heat Fluxes with $\mathrm{H}_{2} \mathrm{O}$

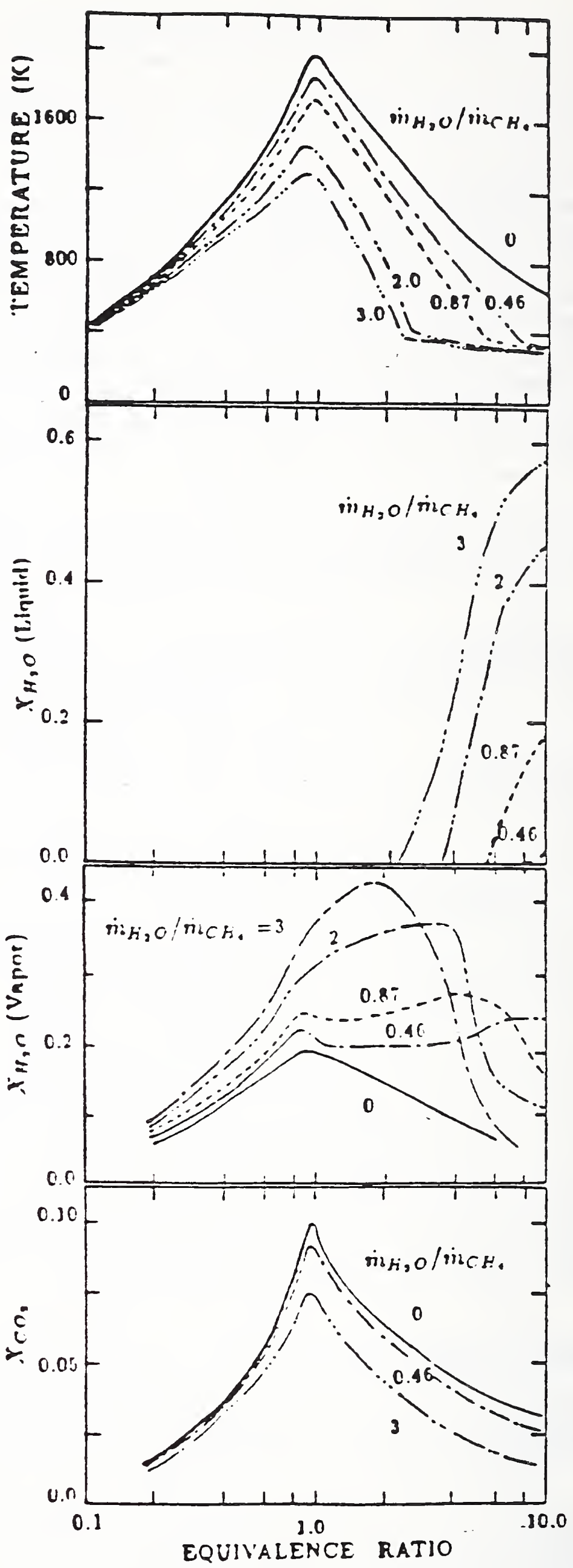

Eis. 3 State Relationships with $\mathrm{H}_{2} \mathrm{O}$ 
CENTER FOR FIRE RESEARCH

OTHER AGENCY PROJECT - 1988

\section{QUICK RESPONSE SPRINKLERS IN OFFICE OCCUPANCIES}

Funding Agency: U.S. Government Services Administration

Project Leader: William D. Walton

Project Objective

To develop a basis for a design criteria for the use of quick response sprinklers in office occupancies.

\section{Technical Accomplishments}

The Center for Fire Research has completed the first three phases of a multiphase reserch project addressing the use of quick response sprinkler technology in office occupancies. The major areas of the first three phases of this project were to: (1) examine the potential applicability of quick response technology to office occupancies; (2) characterize office fuel packages; and (3) measure the effect of sprinkler spray density on suppression. The work to date has demonstrated some of the potential benefits of using quick response technology and provided part of the basis for developing a design standard focusing on the fuel packages and the amount of water required to extinguish these packages. The current phase of the project focuses on the determination of the time of sprinkler actuation under conditions found in office occupancies.

\section{Publications}

Walton, w.d., and Budnick, E.K., Quick Response Sprinklers in Office Configuration: Fire Test Results, Nat. Bur. Stand. (U.S.), NBSIR 88-3695, 1988, January.

Walton, W.D., Suppression of Wood Crib Fires with Sprinkler Sprays: Test Results, Nat. Bur. Stand. (U.S.), NBSIR 88-3696, 1988, January. 
CENTER FOR FIRE RESEARCH

OTHER AGENCY PROJECT - 1988

FOAM FIRE SUPPRESSION

Funding Agency: U.S. Fire Administration

Project Leader: Daniel Madrzykowski

Project Objective:

Examine the fire suppression effectiveness of water-based compressed air foam relative to plain water.

Technical Accomplishments:

Ignition inhibiting and mass retention measurements were completed in which the effectiveness of synthetic hydrocarbon surfactant foam generated with compressed air was compared to plain water spray. Foam provided twice the increase in ignition delay compared to plain water for Tl-11 plywood exposed to a radiative heat flux in the range of $15 \mathrm{~kW} / \mathrm{m}^{2}$ to $60 \mathrm{~kW} / \mathrm{m}^{2}$ for an equal amount of moisture per unit surface area. Measurements also showed that moisture in the form of foam accumulated 20 times faster on vertical surfaces than water sprayed onto the surface. Measurements of fire extinguishing capabilities of foam relative to plain water will be performed next. Waterbased compressed air foam generated with addition of a commercial synthetic hydrocarbon surfactant at low concentrations, typically 0.3 percent by volume, may enhance the performance of limited water supply residential sprinkler systems.

\section{Publications:}

Study of the Ignition Inhibiting Properties of Compressed Air Foam, Daniel Madrzykowski, National Institute of Standards and Technology Interagency Report, NISTIR 88-3880, October 1988. 
CENTER FOR FIRE RESEARCH

OTHER AGENCY PROJECT - 1988

\title{
COMPARTMENT FIRE MODEL TO SIMULATE THE EFFECTS OF
}

ROOF VENTS, SPRINKLERS, AND THEIR INTERACTIONS

\author{
Funding Agency: AAMA Research Foundation \\ Project Leader: Leonard Y. Cooper \\ Douglas Walton, Fire Prevention Engineer \\ William Davis, Physicist
}

\section{Project Objective}

Develop a computer code model for engineering analysis of roof vents, sprinklers, and their interactions during fire-generated environments.

Technical Accomplishments

A complete theory for the model was developed. Coding of the equations has been initiated, and a preliminary working computer program which illustrates some of the features of the expected final product was prepared and demonstrated. Submodel algorithms/subroutines prepared for and tested in this model will be used to enhance future CCFM/RFM products.

\section{Reports and Publications}

"Estimating the Environment and the Response of Sprinkler Links in Compartment Fires with Draft Curtains and Fusible Link-Actuated Ceiling Vents - Part I: Theory," Cooper, L.Y., NBSIR 88-3743, April 1988.

"Estimating the Environment and the Response of Sprinkler Links in Compartment Fires with Draft Curtains and Fusible Link-Actuated Ceiling Vents - An Overview," Cooper, L.Y., presented at the 10th Joint Meeting of the UJNR, and to appear in the Proceedings, Tokyo, June 1988; to be presented at the 1988 Technical Meeting of the Eastern Section of the Combustion Institute, Dec. 57, 1988, and published in Proceedings. 
CENTER FOR FIRE RESEARCH

OTHER AGENCY PROJECT - 1988

\title{
EXPERT SYSTEM
}

\author{
Funding Agency: Air Force \\ Project Leaders: Richard L. Smith, Project Leader \\ Harold E. Nelson, Leader, Fire Safety Engineering Team
}

\section{Project Objective}

To develop a computer program which will make an expert estimate of the fire safety of a general purpose warehouse based on CFR's deterministic physical models, technical data, and expert judgment.

\section{Technical Accomplishments}

The first phase of the project, now completed, focused on the selection of a general purpose warehouse as the first type of building for the expert system to analyze. This was accomplished after an initial study determined that, in general, the knowledge base for an expert system consists of public and private knowledge--the public knowledge for this system being reasonably large and its sources numerous. Therefore, it was determined that the system's knowledge base would be constructed by first including the general public knowledge, followed by the CFR's public knowledge, and finally the private knowledge of our human expert, H. Nelson. (The program will be written on a Symbolics computer (a LISP machine) using state-of-the-art expert system development software tools.)

The second phase, which focusses on the writing of the program, is underway and has resulted in: (1) the completion of an algorithm for protecting a building from external fire exposure; (2) the development of a computer programming procedure using oriented programming techniques to save the state of the reasoning process of the expert system under development; and (3) the development of a capability to draw floor plans (exterior walls, interior walls, doorways, doors, and windows) with understanding.

\section{Reports and Publications}

"ASKBUDJr: A Precursor of an Expert System for the Evaluation of Fire Hazard" by Richard L. Smith, Fire Technology 23, pp. 5-18, February 1987. 


\section{CENTER FOR FIRE RESEARCH \\ OTHER AGENCY PROJECT - 1988}

\section{FIRE RISK ASSESSMENT}

\section{Funding Agency National Fire Protection Research Foundation (NFPRF)}

Project Leader $S$. Wayne Stiefel

\section{Project Objective}

To develop a generally applicable methodology for the proper evaluation of the expected life-safety risk associated with the fire performance of new and existing products.

\section{Technical Accomplishments}

The first phase of the project (now completed) focused on the development of the general methodology and its application to a diverse set of three example cases (upholstered furniture in residences, carpet in offices, and concealed combustibles in hotels). The experience gained through the case studies was used to refine the methodology and begin to evaluate its overall validity. Critical assumptions were also tested through sensitivity analyses.

The second phase of the work is now underway. Here we will continue the evaluation and refinement of the methodology through further sensitivity analyses and the conduct of a fourth case study - interior finish in restaurants. A portion of the work in phase 2 will involve an attempt to reduce the computational burden of the method by the substitution of procedures which eliminate unwarranted complexities in specific situations (e.g., limiting the use of behavioral models to certain occupancies where occupant interactions are known to be important to the eventual outcome).

Another crucial activity of phase 2 is the public documentation of the work completed in phase 1 . This will consist of a detailed report on the methodology, and the documentation of the three case studies.

The final phase of the program will produce the user documentation necessary to transfer the method and the computer software which implements it to the international fire protection community. From preliminary indications, it is expected that its application to real world problems will be immediate.

\section{Publications}

Bukowski, R. W. and Stiefel, S. W., An Overview of a Method for Predicting Fire Risk, NISTIR, in press.

\section{$\underline{\text { Related Grant }}$}

University of Calif. (LA), G. Apostolakis, Fire Risk Analysis. 


\title{
CENTER FOR FIRE RESEARCH \\ NATIONAL BUREAU OF STANDARDS \\ FY 88
}

\section{Institution: University of California at Los Angeles \\ Grant No.: 60NANB6D0649}

Title: Fire Risk Analysis Methodology

Principal Investigator: Professor George Apostolakis

Department of Mechanical, Aerospace

and Nuclear Engineering

University of California

5532 Boelter Hall

Los Angeles, CA 90024-1597

\author{
Other Professional Personnel: M.D. Brandyberry, Ph.D. student \\ NBS Scientific Officer: Mr. Wayne Stiefel
}

\section{Technical Abstract:}

Introduction To analyze a building fire scenario from initial ignition to its consequences, e.g., dollar losses, injuries, deaths, a necessary input is the frequency of the initiating event (IE). For many materials, objects, building types, etc., this frequency of ignition may be obtained from data on fires which have occurred. A problem of interest, however, is what happens to this frequency of ignition if the product is changed in some way (furniture covered in a new fabric, plastic composition changed, etc.). Changes in a product may also affect other portions of a fire scenario such as secondary ignitions or effects from changed products of combustion. The objective of this project is to develop a methodology for evaluating the changes in initiating event characteristics which may be expected due to changes in a product under analysis.

Probabilistic Interpretation of Ignition The frequency of ignition of an item in a compartment in a building is composed of two basic elements:

- The frequency with which an item is exposed to an ignition source (fr(exposure)).

- The fraction of times that the item is ignited given exposurc to the ignition source (fr(ignition / exposurc)). This factor depends on

1. The physical characteristics of the item (heat transfer propertics, ignition propertics).

2. The intensity and duration of the ignition source. (Is the source removed or docs it sclf extinguish before ignition? Is the heat flux at the target adcquatc to ignite it within the exposurc duration?).

The simple equation combining these is: 


$$
\mathrm{fr}(\text { ignition })=f(\text { (exposure }) \times \text { fr(ignition } / \text { exposure })
$$

To predict the frequency of ignition of a new product, both of the factors on the right must be known. There are, however, no direct statistics on the frequency of exposure of any product to an ignition source (this factor includes both exposures which have caused fires and those which have not.) Also for a brand new product, there will be no directly applicable evidence for the ignition of an item given exposure to an ignition source, but evidence from similar products and from mathematical modeling and experimental testing may be used.

Radiant Ignition of Products by Heating Appliances To put the problem into perspective, we have chosen the class of ignition sources "heating appliances" as an example to illustrate the problems involved. This class of ignition sources can ignite materials by a number of different methods. Heat conduction by contact with the appliance, radiation transfer by the target being too close to the target, and other problems such as spilled kerosene from a portable liquid fueled heater igniting are all methods by which a heating appliance may ignite a material.

We may limit our attention, however, to those ignition scenarios in which the frequency of ignition may change due to the effects of a new material. This means that such scenarios as those involving spilled kerosene are not of interest to us since many (if not most) combustible materials will ignite if doused with kerosene and ignited and changing the materials properties will have little effect on the ignition frequency of the material due to this scenario. (Other analyses, such as analyzing the effects of changes in human behavior when filling the heater or a new heater fuel tank design may change the frequency, but we are not considering these here.) After studying the heater fire problem and limiting ourselves to consumer products (as . opposed to structural materials), we have concluded that the major mode of change in the frequency of product ignition due to heating appliances will be through changes in resistance to radiant ignition of the target product. This is the type of scenario that we analyze.

Radiant ignition of a material by a heating appliance occurs when the material is placed too close to that appliance or vice versa (if the appliance is portable). To assess the frequency of exposure of the material to radiant heat flux from a heater, we must assess how often the material will come into proximity to a heater by whatever means. There are, as noted before, no direct statistics on this type of frequency, and thus we must infer the value from available evidence. The result will not be a single value, but will necessarily be a probability distribution.

Three sources of information have been identified as pertinent to the problem:

1. Reported fires caused by heating appliances [1].

2. Estimates of unreported fires caused by heating appliances [2].

3. Estimates of accidents involving consumer products $[3,4]$.

The number of reported fires can be interpreted to be a lower bound on the frequency of exposure of a heating appliance to a combustible (by considering a suitable portion of the total number of heater fires; e.g., those caused by being "too close" to the heater). We may utilize an estimate of the number of unreported heater fires caused by "human 
carelessness" as a better estimate of the number of fires actually caused by being "too close" to the heater (this number probably also contains kerosene spills and other such accidents). The third value we have considered is the number of accidents involving consumer products, restricted to those accidents in which the product did not directly cause the accident (e.g., accidents involving chain saws were not included). This value may be considered to be the frequency with which people do "incorrect things" with consumer products and thus may be considered to be applicable to the act of a person "putting a combustible too close to a heater" or visa versa. The above considerations lead us to a lognormal distribution for the exposure frequency as shown in Figure 1.

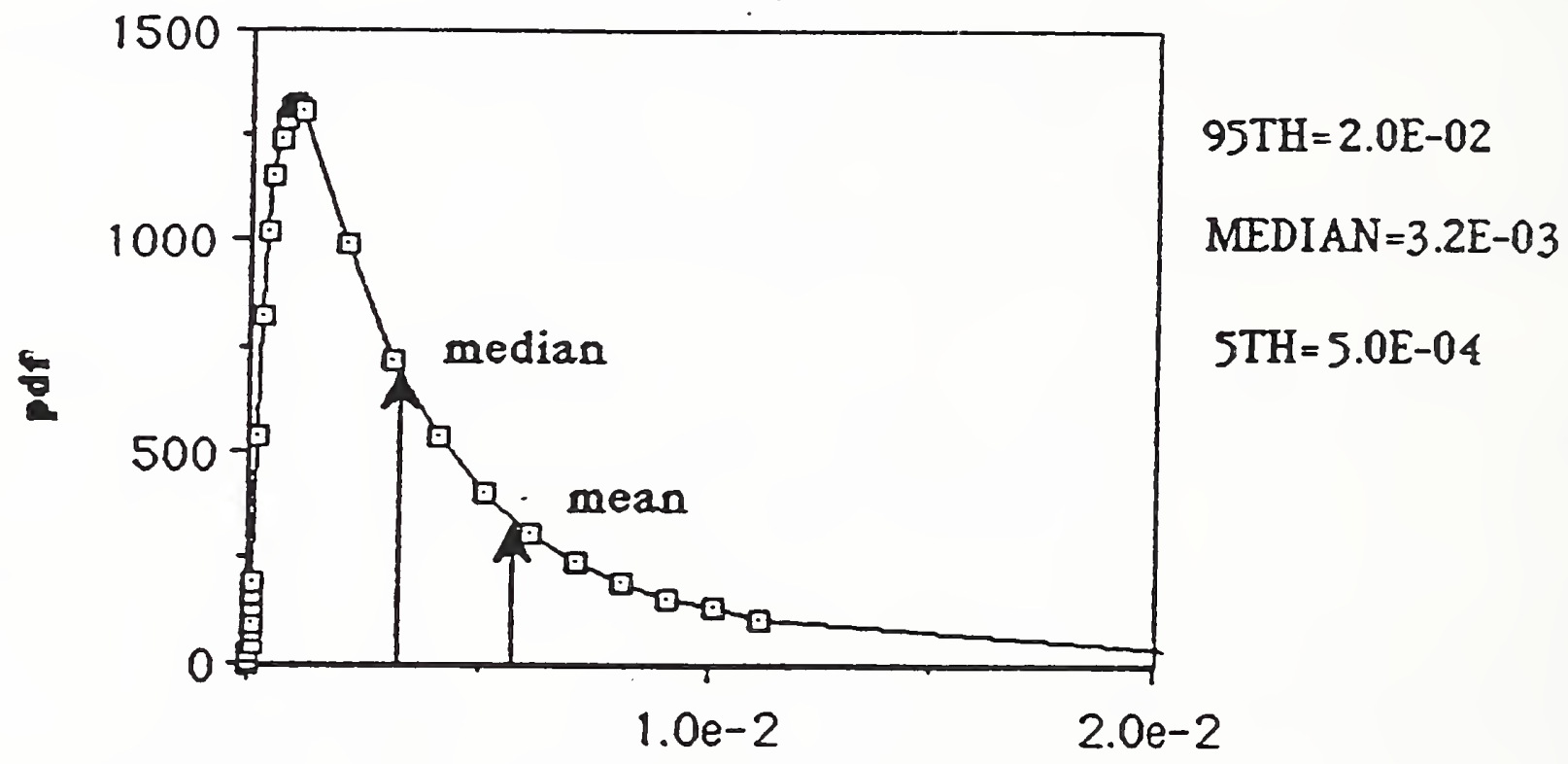

Figure 1. Distribution of the frequency of exposure (events per year per household)

Ignition factor The second factor, fr(ignition / exposurc), is scenario and product dependant. For a specified product under analysis, there will be certain characteristic thermophysical properties of the material which will control heat transfer within the solid (density, thermal conductivity, surface emissivity). The solid will also have some specific conditions under which it will ignite, e.g., critical surface temperature, critical heat flux, etc. [5]. Additionally, there will be a set of environmental conditions which will affect heat transfer to and from the target solid (e.g., environment temperature, radiant heat flux to the target from external sources, etc.) For the heater fire problem, several numerical tools are under development with which it will be possible to estimate the geometrical conditions under which a solid cxposed to radiant flux from a heater may ignite. As these tools will be deterministic in nature (computer models), they will be subject to uncertainties in both the parameter inputs and in the models thenselves. This will lead to distributions of the distance of the target from the source under which ignition is possible. Distributions of the spatial heat flux in front of a flat-plate radiator are currently under study (see Figure 2., a preliminary result for a fireplace.) 


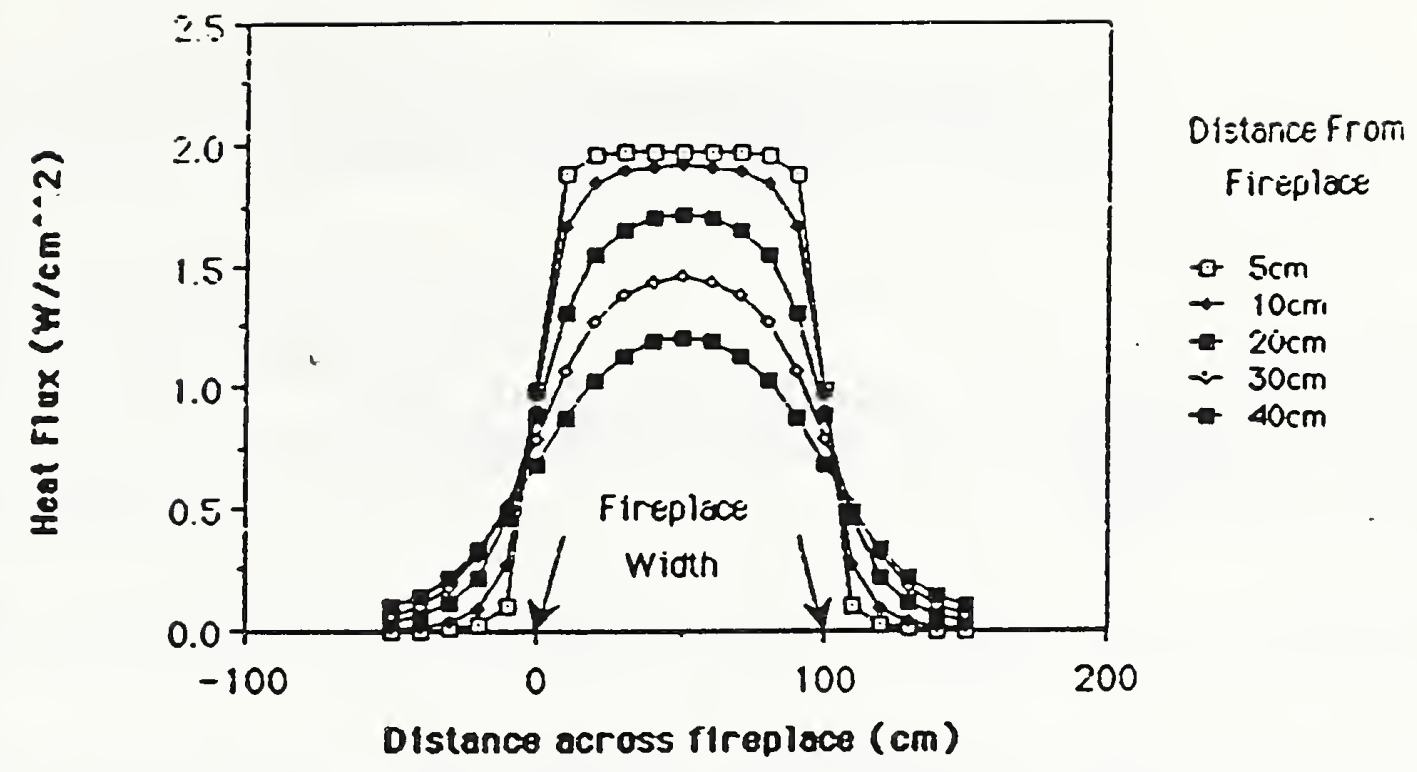

Figure 2. Heat flux at $40 \mathrm{~cm}$ height in front of a $100 \times 80 \mathrm{~cm}$ flat plate uniformly radiating $16 \mathrm{~kW}$

\section{REFERENCES}

1. Hall, J.R., "Update on the Auxiliary Heating Fire Problem," Fire Journal, 80, (2), 52-62, March, 1986.

2. 1984 National Sample Survey of Unreported Residential Fires, Final Technical Report to US Consumer Product Safety Commission (Princeton, New Jersey: Audits \& Surveys, Inc., June 1985).

3. Accident Facts, 1987 Edition, National Safety Council.

4. Vital Statistics of the United States in 1984, Center for Vital and Health Statistics, 1987.

5. Gandhi, P.D., Kanury, A.M., "Criterion for Spontaneous Ignition of Radiantly Heated Organic Solids, Combustion Science and Technology 50, 233-254, 1986. 
APPENDIX

ALPHABETICAL LISTING OF

CENTER FOR FIRE RESEARCH GRANTS

$\underline{\text { Page }}$

Brown University

A Study of the Effects of Oxygen Transport and

Temperature History on the Chemistry of PMMA and

Cellulose Pyrolysis

Brown University

Soot Morphology in Flames

California Institute of Technology

Experimental Study of Environment and Heat Transfer in a Room Fire

Clemson University

Graphical Presentation and Numerical Analysis of

Fire Data for Model Validation

Clemson University

Numerical Analysis Support for Compartment Fire

Modeling Code Development

Factory Mutual Research Corporation

Prediction of Fire Dynamics

Task 1. Prediction of Fires in Buildings

The George Washington University

Chemical Pathways to Soot Formation in Diffusion

Flames

Marquette University

Thermal Stabilization of Polymers by Cross-Link

Formation

Michigan State University

Effect of Water on Ignition of Cellulosic Materials

The Pennsylvania State University

Soot Particle Formation and Destruction in Diffusion Flames

The Pennsylvania State University

Upward Flame Spread on Vertical Wall

Rutgers - The State University of New Jersey

Negatively Buoyant Flows Generated in Enclosure Fires

by the Downward Turning of Ceiling Jets 
Southwest Research Institute

Analysis of Hazards to Life Safety in Fires: A

Comprehensive Multi-Dimensional Research Program--

Part 3

TRW Space and Technology Group

48

Wind-Aided Flame Spread

University of California, Berkeley

Fire Modeling

University of California, Berkeley

Fire Propagation in Concurrent Flows

University of California, Berkeley

Flame Radiation

University of California, Los Angeles

Fire Risk Analysis Methodology

University of California, Riverside

Expert System Approach for Spectra/Structure

Correlation for Vapor-Phase Spectra

University of California, Santa Barbara

A Study of the Suppression and Extinction of

Fires by Water Sprays

University of Dayton Research Institute

162

Mathematical Modeling of Furniture Fires

University of Kentucky

A Study of Oil-Slick Combustion

University of Maryland, Baltimore

Fire Environment in Counterflow Ventilation

University of Maryland, College Park

An Investigation of Simulated Oil Well

Blowout Fires

University of Maryland, College Park

Development of a Technique to Assess the Adequacy

of the Municipal Water Supply for Residential

Sprinkler Systems

University of Maryland, College Park

Interaction of Radiation and Conduction in Polymeric Materials 
University of Maryland, Sollege Park

Kinetics of Aggregate Formation

University of Maryland, College Park

Transient Ceiling Jet Characteristics

University of Maryland, College Park

Transient Cooling of a Hot Surface by

Droplets Evaporation

University of Michigan

Radiation from Turbulent Luminous Flames

University of Montana

Kinetics of Oxygen Chemisorption and Desorption

of Combustion Products in the Smoldering Combustion of Cellulosic Material

University of Pittsburgh

Toxicity of Plastic Combustion Products

Virginia Polytechnic Institute and State University

Compartment Fire Combustion Dynamics 
"Summaries of Center for Fire Research In-House Projects and Grants - 1988"

AUTHOR(S)

Sonya M. Cherry (Editor)

PERFORMING ORGANIZATION (If joint or other thon NBS, see instructions)

7. ContracVGrant No.

NATIONAL BUREAU OF STANDARDS

U.S. DEPARTMENT OF COMMERCE

8. Type of Report \& Period Covered GAITHERSBURG, MD 20899

SPONSORING ORGANIZATION NAME AND COMPLETE ADDRESS (Street, City, Stote, ZIP)

Document describes a computer program; SF-185, FIPS Software Summary, is attached.

ABSTRACT (A 200-word or less factual summary of most significant information. If document includes a significant bibliography or literature survey. mention it here)

This report describes the research projects performed in the Center for

Fire Research and under its grants program during FY1988.

KEY WORDS (Six to twelve entries; alphabetical order; capitalize only proper names; and separate key words by semicolons)

cellulose; charring; combustion; fire models; flame spread; ignition; polymers; smoke; soot; toxicity

AVAILABILITY

X Unlimited

For Official Distribution. Do Not Release to NTIS

Order From Superintendent of Documents, U.S. Government Printing Office, Washington, D.C. 20402.

X Order From National Technical Information Service (NTIS), Springfield, VA. 22161
14. NO. OF

PRINTED PAGES

$\frac{226}{15 . \text { Price }}$

$\$ 24.95$ 


\section{,}



(3) 
